(mean 3.6\%, range $0.6-8.4 \%$ ) and metastatic carcinoid tumor (mean $11.5 \%$, range $1.3-46.7 \%)(\mathrm{p}=0.0019)$. The survival time was much shorter in patients with metastatic carcinoid tumor (median survival 5.8 years) comparing to those with primary ovarian carcinoid tumor (median 14.2 years) $(\mathrm{p}=0.0005)$. A strong association between Ki67 index and patient survival time was identified (Hazard ratio 1.11, $\mathrm{p}=0.001$ ).

Conclusions: Comparing to primary ovarian carcinoid tumor, metastatic carcinoid tumor usually exhibits a higher Ki67 index and a worse outcome. Ki67 index is significantly associated with the survival time in patients with carcinoid tumor involving ovary.

\section{Gastrointestinal Pathology}

621 Tubulovillous Adenomas with Serrated Features Are Precursors to KRAS Mutant Colorectal Carcinoma

Amrou Abdelkader, Christopher Hartley, Catherine Hagen. Medical College of Wisconsin, Milwaukee, WI; University of Wisconsin Hospital and Clinics, Madison, WI. Background: Conventional adenomas and serrated polyps represent precursor lesions to two separate pathways of colorectal carcinoma. Occasionally, tubulovillous adenomas (TVA) show focal features resembling traditional serrated adenomas, including ectopic crypt foci, luminal serration, and cytoplasmic eosinophilia. The biologic significance and molecular findings associated with this histologic variant of TVA are largely unknown. The goal of this study was to investigate the frequency of KRAS and BRAF mutations in TVA with serrated features (sTVA) in comparison to a control group of TVA. Design: 27 consecutive sTVA and a control group of 27 TVA matched for highest degree of concurrent dysplasia/carcinoma were evaluated. Specimens were analyzed for mutations in KRAS codon 12/13 and BRAF codon V600E; other variants in KRAS and BRAF were not evaluated in this study. Somatic variants in KRAS and BRAF were identified by dideoxy sequencing (assay limit of detection 15\%).

Results: The mean age of the sTVA group was 68.9 (M:F 1.5:1) and mean age for the TVA group was 63.0 (M:F 1:1.1). All 27 TVA were wild type for BRAF mutation. One sTVA (3.7\%) was positive for the BRAF V600E mutation. KRAS analysis was completed on 22 TVA and 24 sTVA. sTVA were significantly more likely to harbor KRAS mutations $(66.7 \%$ vs. $18.2 \%, \mathrm{p}=0.001)$. A summary of the KRAS mutations is shown in Table 1. When cases in which KRAS testing was not completed were excluded, there was still no significant difference in the distribution for the highest grade of concurrent neoplasia in the sTVA and TVA groups $(\mathrm{p}=0.67)$.

\begin{tabular}{|l|l|l|}
\hline Table 1: Summary of KRAS mutations in TVA and sTVA \\
\hline KRAS 12/13 Genotype & TVA & sTVA \\
\hline G12D & $0(0 \%)$ & $4(16.7 \%)$ \\
\hline G12R & $1(4.5 \%)$ & $0(0 \%)$ \\
\hline G12S & $0(0 \%)$ & $1(4 \%)$ \\
\hline G12V & $1(4.5 \%)$ & $5(21 \%)$ \\
\hline G13C & $1(4.5 \%)$ & $1(4 \%)$ \\
\hline G13D & $1(4.5 \%)$ & $5(21 \%)$ \\
\hline WT & $18(82 \%)$ & $8(33.3 \%)$ \\
\hline Total: & 22 & 24 \\
\hline Legend: TVA=tubulovillous adenoma; sTVA=TVA with serrated features; WT=wild type \\
\hline
\end{tabular}

Conclusions: We confirm results of few prior studies showing more frequent KRAS mutation in STVA versus TVA. However, we addressed the shortcoming of prior studies by controlling for degree of associated dysplasia/carcinoma in our design. KRAS mutation is a molecular correlate of the mixed histology of sTVA and may explain the literature's tenuous data suggesting a higher risk of advanced outcomes in these polyps.

$622 \quad$ Age Less Than Forty Is Predictive of Mismatch Protein Loss in Colorectal Tubulovillous Adenomas

Sohaib Abu-Farsakh, Danielle Marino, Arthur DeCross, Qi Yang, Loralee McMahon, Jennifer J Findeis-Hosey. University of Rochester Medical Center, Rochester, NY. Background: Lynch Syndrome (LS) is a genetic syndrome driven by germline mutations in mismatch repair (MMR) protein complexes which result in an increased risk of numerous neoplasms. Mutations are most frequently encountered in the $M L H 1, M S H 2$, MSH6, or PMS2 genes. Immunohistochemical (IHC) testing using antibodies against the MMR proteins has been well documented for LS screening in CRC specimens; however, few studies have examined the use of MMR IHC screening in the general population before the development of frank malignancy. Here we aim to explore the possibility of LS screening in patients $<50$ years old who have adenomas with villous features. Design: Our surgical pathology archives were searched for cases of colorectal adenomas with villous features from 1/1/1997-5/31/2016 in patients $<50$ years old. Patients with a known history of CRC, LS, or familial adenomatous polyposis were excluded. Slides were reviewed and those that did not meet strict criteria for villous morphology were excluded. Sixty-nine cases were identified in patients $<45$ years old and were compared to a cohort of 27 cases of patients $45-50$ years old recieved between 1/1/2013 and 7/31/2015. All 96 cases underwent IHC testing with antibodies against MSH6 and PMS2. Cases with abnormal staining for either of these proteins underwent additional IHC testing with antibodies for MLH1 and MSH2. Abnormal staining was defined as less than $10 \%$ of adenomatous cells exhibiting positive nuclear staining.

Results: Abnormal MMR IHC staining was identified in four out of 31 cases $(13 \%)$ in patients less than 40 years old and in one out of 38 cases $(3 \%)$ in those $40-45$ years old. None of the 27 cases from those 45-50 years demonstrated loss in MMR staining. Of the five cases that showed MMR abnormalities, two showed isolated loss of MSH6, one showed isolated loss of PMS2, one showed loss of MSH2 and MSH6, and one showed loss of MLH1 and PMS2. The difference in the MMR loss rate by IHC between the three groups approaches statistically significant $(\mathrm{p}=0.065)$.

Conclusions: MMR IHC testing of CRCs has been well described; however, testing of adenomas has only been described previously in limited fashion and when done has been enriched for patients with LS. Here we identified a propensity for polyps with villous features from younger patients to display MMR IHC abnormalities. These results suggest that MMR IHC testing of adenomas with villous features in patients less than 40 years old may assist with the identification of LS.

623 Clincopathologic Studies of PD-L1 Expression on Esophageal Adenocarcinoma and Squamous Cells Carcinoma

Sohaib Abu-Farsakh, Amy Lalonde, Tongtong Wu, Zhongren Zhou. University of Rochester, Rochester, NY.

Background: Programmed death ligand 1 (PD-L1) is a ligand for the inhibitory programmed death receptor 1 (PD-1). Induction of PD-L1 expression on tumor cells leads to the inhibition of immune responses against cancer. Therefore, blockage of PD-1 or PD-L1 can activate the anti-tumor activity. Our study investigated the PD-L1 immunohistochemistry in both esophageal adenocarcinoma (EAC) and squamous cell carcinoma (ESCC).

Design: TMA of $101 \mathrm{EAC}$ and 27 ESCC were immunohistochemically stained for PDL1. Expression of PD-L1 was scored for positive, negative and percentage $(0-100 \%)$ for positive cells. The data were analyzed byPearson Correlation.

Results: Of 101 AC cases, 12 (12\%) were positive for PD-L1 immunostain. Of 27 SCC cases, $6(22 \%)$ were positive for PD-L1 immunostain. The PD-L1 expression in EAC were significantly correlated with female, but not correlated with other clinicopathologic features including age, differentiation, lymph nodes, survival and staging. The mean and median of the percentage of PD-L1 positive tumor cells is $43 \%$ and $35 \%$ in EAC and is $21 \%$ and $15 \%$ in ESCC. The PD-L1 expression is not significantly associated with proliferation index Ki67, MCM4 and MCM7.

Conclusions: PDL-1 expression is relative low in EAC (12\%) and ESCC (22\%). PD-L1 expression is significantly associated with female.

624 Prevalence of Immunohistochemically Demonstrable Helicobacter Pylori in Biopsies without Histologic Evidence of Gastritis Andres Acosta, Michael R Pins. University of Illinois at Chicago, Chicago, IL; Chicago Medical School, Chicago, IL; Advocate Lutheran General Hospital, Park Ridge, IL. Background: Helicobacter pylori is a major cause of gastritis, with virtually all infected patients demonstrating moderate to severe acute and/or chronic inflammation. In fact, the presence of the bacteria in otherwise normal gastric biopsies is believed to be rare. Since we perform reflexive immunohistochemistry (IHC) on all gastric biopsies with a requisition request to rule out $\mathrm{H}$. pylori, we decided to study the prevalence of immunohistochemically demonstrable H. pylori in the absence of inflammation (HPy+/Inf-).

Design: We reviewed gastric biopsies with reflexive H. pylori IHC performed in our institution between 2007 and 2015. HPy+/Inf- biopsies were selected on the basis of the presence of antral mucosa, undetectable or minimal nonspecific inflammation and a positive IHC result. All IHCs were performed in the same laboratory under the same protocol.

Results: A total of 15,460 gastric biopsies with IHC for H. pylori were evaluated and six $\mathrm{HPy}+/$ Inf- cases were initially identified. Of these, two were later demonstrated to represent $\mathrm{H}$. heilmannii by IHC $(2 / 6=33 \%)$, making the prevalence of true HPy+/Infcases $0.0003 \%$ (3/10,000 biopsies). All HPy+/Inf- cases were women, with a median age of 31 yrs (range 4-66 yrs). Of these, one had a history of previously treated $\mathrm{H}$. pylori gastritis 4 years prior to the current biopsy. The youngest patient had type $2 \mathrm{Gm} 1$ gangliosidosis with functional immunosuppression, and the remainder two patients did not have significant comorbidities.

Conclusions: The prevalence of $\mathrm{H}$. pylori in the absence of inflammation is extremely low $(0.0003 \%)$, making inflammation a good surrogate for the presence of the bacteria, and an effective trigger for IHC studies when the organism is not readily identifiable on $\mathrm{H} \& \mathrm{E}$ slides. Reflexive IHC upon a request to rule out $\mathrm{H}$. pylori may be justified in some institutions for other reasons, such as improved turnaround time. Additionally, IHC might also be considered in biopsies without significant inflammation when there is a history of treated $\mathrm{H}$. pylori gastritis or immunosuppression. Also, in our experience, $33 \%$ of $\mathrm{HPy}+/$ Inf- cases actually represented $\mathrm{H}$. heilmannii infection, thus justifying the immunohistochemical confirmation of this organism in the context of a suggestive bacterial morphology. Our data suggest that neither H. pylori "colonization" nor $\mathrm{Hpy}+$ / Inf- purely as a result of the vagaries of random sampling ("sampling error") occur.

625 Molecular Lymph Node Staging in Rectal Carcinomas: Can It Be Performed?

Iban Aldecoa, Carla Montironi, Natalia Rakislova, Dulce Momblan, Josep Antoni Bombi, Francesc Balaguer, Antoni Castells, Antonio Lacy, Miriam Cuatrecasas. Hospital Clinic - University of Barcelona, Barcelona, Spain

Background: Pathologic hematoxylin and eosin (H\&E) lymph node (LN) staging in colorectal carcinomas (CRC) may not provide robust prognostic data, as up to $20 \%$ of stage II CRC patients recur from disease within 5 years of intended curative surgery. Recent studies have addressed that molecular staging in colon carcinomas using CK19 mRNA can correlate with classical high risk factors, and there is increasing evidence that it can be used on a daily basis. Nevertheless, there is lack of data regarding the use of this technique in rectal carcinomas. 
Design: Tumor burden LN detection was performed with the reverse transcription loopmediated isothermal amplification for CK19 mRNA. A total of $493 \mathrm{LN}$ were harvested from 29 rectal resections without neoadjuvant treatment. In each case, several LN were pooled together into PCR tubes for molecular analysis.

Results: The median age of the patients was 68 years, with a male:female ratio of 1.4:1. The pTN stages were: 8 pT1, 9 pT2, 8 pT3 and 4 pT4; 23 pN0, 3 pN1a, 2 pN1b and 1 $\mathrm{pN} 2 \mathrm{a}$. A median of $15 \mathrm{LN}$ were dissected per specimen. Using pooling method for LN analysis, a median of 2 molecular assays were performed per case. Twelve out of 29 cases contained tumoral CK19 mRNA in the lymph nodes, with a median 6300 copies/ $\mu \mathrm{L}$ (IQR 470-30,675). Molecular positivity was correlated to some classical high risk factors such as tumor budding, neural invasion and vascular invasion (all $\mathrm{p}<0.05$ ). A tendency was observed between CK19 mRNA LN positivity and tumor grade.

Conclusions: Molecular LN staging in rectal carcinoma correlates with some classical high risk factors and can become more accurate than conventional H\&E staging. It is a feasible method in daily practice and requires few molecular determinations per case.

626 Autoimmune Polyendocrinopathy-Candidiasis-Ectodermal Dystrophy (APECED) Associated Gastritis - A Variable Phenotype

Michael Allgaeuer, Elise MN Ferré, Theo Heller, Michail Lionakis, Martha M Quezado. NCI, NIH, Bethesda, MD; NIDDK, NIH, Bethesda, MD; NIAID, NIH, Bethesda, MD. Background: APECED is a rare condition caused by a mutation in the autoimmune regulator (AIRE) gene. It manifests with chronic mucocutaneous candidiasis and multisystem autoimmunity most commonly leading to hypoparathyroidism and adrenal insufficiency. Patients frequently develop hepatitis, gastritis, intestinal dysfunction, and vitamin B12 deficiency. So far the histopathological features of gastritis remain poorly defined.

Design: Available gastric biopsies from 24 patients with a confirmed diagnosis of APECED (biallelic AIRE mutation $(21 / 24 ; 87.5 \%)$ or clinical criteria $(3 / 24 ; 12.5 \%)$ ) enrolled in a natural history study were systematically assessed for the presence of inflammation, eosinophilic infiltrates, intestinal metaplasia, dysplasia, carcinoma, neuroendocrine hyperplasia and H. Pylori organisms.

Results: Sixty-eight gastric biopsies were reviewed by an experienced gastrointestinal pathologist (M.M.Q.) and pathologist-in-training (M.A.). A median of 2 biopsies was obtained per patient (range 1-14) in a median of 1 upper endoscopy (1-13). Mean age at first procedure resulting in a diagnosis of significant pathology was 22.7 years (3-55) with $16(67 \%)$ female and $8(33 \%)$ male patients. Inflammation was present in $76.5 \%$ of biopsies examined $(52 / 68)$. Inactive chronic gastritis was present in 40 samples and was mild in $33(33 / 68,48.5 \%)$, moderate in $6(6 / 68,8.8 \%)$, and severe in $1(1 / 68,1.5 \%)$. Active chronic gastritis was seen in 8 samples $(8 / 68,11.8 \%)$. Autoimmune atrophic gastritis (AIG) was confirmed in 4 samples $(4 / 68,5.9 \%)$ of two patients with one sample displaying linear neuroendocrine cell hyperplasia. All biopsies were negative for $\mathrm{H}$. pylori. Eosinophils were commonly seen but increased (>15/1HPF) in 1 sample only $(1 / 68,1.5 \%)$. Metaplastic changes were mostly absent $(59 / 68,86.8 \%)$. The remaining biopsies showed complete intestinal metaplasia, either focal $(6 / 68,8.8 \%)$ or prominent $(3 / 68,4.4 \%)$. Dysplasia was not identified in the large majority of cases $(67 / 68$, $98.5 \%$ ). One biopsy was positive for intramucosal signet ring cell adenocarcinoma in a background of AIG. Biopsies of that patient 28 months prior showed mild chronic inactive gastritis with focal complete intestinal metaplasia.

Conclusions: Chronic gastritis with and without intestinal metaplasia is a common finding in patients with APECED and displays various phenotypes including autoimmune atrophic gastritis. The occurrence of adenocarcinoma appears to be a rare phenomenon, but close follow-up with multiple biopsies may be warranted.

\section{Lack of Prognostic Significance of HER2 Expression Profile} in Colorectal Carcinoma

Jaudah A Al-Maghrabi, Abdelbaset Buhmeida, Mourad Assidi, Basim J Al-Maghrabi, Ashraf Dallol, Adeel Chaudhary, Adel Abuzenadah, Mahmoud S Al-Ahwal, Mohammed H Al-Qahtani. Faculty of Medicine, King Abdulaziz University, Jeddah, Saudi Arabia; King Abdulaziz University, Jeddah, Saudi Arabia.

Background: Traditionally, the utilization of multimodal treatment approach for colorectal carcinoma (CRC) has been directed by the TNM staging system. However, the conventional staging system, regrettably, is unable to predict the outcome of patients within the same stage. Therefore, finding additional molecular markers that could help in pinpointing the survival outcome and/or treatment response of a group of patients within the same stage will be very helpful towards better management and personalized treatment. The prognostic significance of HER2 gene in CRC is not clear and remains controversial. The objective of this study is to evaluate the association between HER2 amplification/over-expression profile and survival outcome of Saudi CRC patients.

Design: A tissue microarray (TMA) was constructed to contain a total of 243 CRC Formalin Fixed Paraffin Embedded (FFPE) samples of consent patients and stained by immunohistochemistry (IHC) and bright-field dual in situ hybridization (BDISH) methods. The expression patterns of HER2 protein status was correlated to HER2 gene amplification status and assessed for its prognostic value.

Results: The expression profile of $99 \%$ samples showed cyotplasmic expression patterns of different categories, while, only $1 \%$ showed strong (+3) membranous expression pattern of HER 2 protein and those later samples showed a corresponding amplification of HER 2 gene status $(>2)$. However, the cytoplasmic HER2 protein status did not show any significant correlation with different clinico-pathological features and survival outcomes except with age $(\mathrm{p}<0.04)$ and tumor size $(\mathrm{p}<0.03)$.

Conclusions: The study revealed that there was no correlation between both membranous and/or cytoplasmatic HER2 expression and patient's survival outcome either disease-free and/or disease-specific survival among CRC patients.
628 PD-L1 Expression in IBD-Associated Colorectal Cancer and Its Relationship to MSI Status, the PI3K Pathway, and HLA-DR Expression

Lindsay Alpert, Lindsay Yassan, Christopher Weber, Shu-Yuan Xiao, John Hart, Thomas Krausz, Jeremy Segal, Namrata Setia. University of Chicago, Chicago, IL.

Background: Programmed death-ligand 1 (PD-L1) expression is rare in colorectal cancers (CRCs), but a subset of tumors with microsatellite instability (MSI), medullary morphology, and/or increased tumor-infiltrating lymphocytes are known to more frequently express this immunoregulatory molecule. Given the immune-activated background in which inflammatory bowel disease-associated CRCs (IBD-CRCs) arise, the potential relationship between these tumors and PD-L1 expression is intriguing. Since the PI3K pathway is a downstream effector of PD-1-activated T cells and genes in this pathway (PTEN, PIK3CA) are frequently mutated in MSI tumors, the mutational status of such genes in IBD-CRCs is also of interest. Additionally, aberrant expression of HLA-DR was recently identified as a marker of response to anti-PD-L1 therapy in melanoma.

Design: IBD-CRCs resected between 1/2000-7/2015 were identified from the pathology archives. IHC for mismatch repair (MMR) proteins, PD-L1, and HLA-DR was performed on all cases with tumor-containing blocks available. PD-L1 and HLADR expression in tumor cells were scored according to previously described criteria. PIK3CA and PTEN mutational status was assessed using a targeted next-generation sequencing panel aimed at 50 cancer-related genes.

Results: Sixty-two patients with a total of 72 tumors were identified. Five of 58 cases tested $(8.6 \%)$ were positive for PD-L1, while 32 of 56 cases tested $(57.1 \%)$ were positive for HLA-DR. None of the PD-L1-positive cases had a mucinous or medullary morphology. PD-L1 positivity was not significantly associated with any other clinicopathologic characteristics. All 5 PD-L1-positive cases expressed HLA-DR. PIK3CA and/or PTEN mutations were detected in 11/56 tumors tested (19.6\%). Loss of $\geq 1$ MMR protein was seen in 3/67 tumors tested (4.5\%), all of which also exhibited $P I K 3 C A$ and/or PTEN mutations. There was no significant association between PD-L1 positivity and $P I K 3 C A / P T E N$ mutations or MSI status $(\mathrm{p}=0.16$ and $\mathrm{p}=0.24$, respectively) Conclusions: $\mathrm{PD}-\mathrm{L} 1$ positivity in IBD-CRCs is rare, but the frequency is similar to that reported in non-IBD-CRCs. Expression of PD-L1 is not associated with MSI status or PI3K pathway mutations in these tumors, so PD-L1 immunotherapy trials for select microsatellite stable IBD-CRCs should be considered. The findings also support the presence of alternate mechanisms of PD-L1 expression, such as IFN-gamma induced upregulation. Finally, IBD-CRCs that are positive for PD-L1 also express HLA-DR, which may be an indicator of response to therapy.

629

Cancer

Targeted Mutational Analysis in IBD-Associated Colorectal

Lindsay Alpert, Lindsay Yassan, Sabah Kadri, Ibro Mujacic, Nifang Niu, David Montez, Filippo D Galbo, Michelle N Wurst, Chao J Zhen, Christopher Weber, Shu-Yuan Xiao, John Hart, Jeremy Segal, Namrata Setia. University of Chicago, Chicago, IL.

Background: Patients with inflammatory bowel disease (IBD) are at increased risk of developing colorectal carcinoma (CRC). Molecular distinctions between IBD-associated CRCs (IBD-CRCs) and non-IBD-CRCs have been noted, including a decreased likelihood for IBD-CRCs to exhibit microsatellite instability (MSI). Recently, a study comparing mutations in cancer-related genes between IBD- and non-IBD-CRCs revealed further genomic differences (R Yaeger Gastroenterology, 2016). In the current study we investigated the mutational profiles of our institution's IBD-CRCs and compared them to published TCGA data.

Design: IBD-CRCs resected between 2000 and 2015 were identified from the pathology archives. All cases with sufficient FFPE material available underwent molecular testing via a 50-gene hot-spot solid tumor panel utilizing the Ion Ampliseq Cancer Hotspot Panel v2 primer set (Thermo Fisher Scientific) for amplification of 207 hotspot targeted amplicons in a single pool. Data was analyzed using a custom-developed in-house clinical pipeline, and all variants were annotated using Alamut Batch 1.4.4 software (http://www.interactive-biosoftware.com/).

Results: We identified 72 IBD-CRCs occurring in 62 patients; molecular testing was successful in 56 cases. The mean duration of IBD at cancer diagnosis was 20 years (SD 11 years) and did not differ significantly according to the type of IBD present $(\mathrm{p}=0.09)$. The frequency of mutations occurring in $>5 \%$ of all IBD-CRCs (including 3 cases of indeterminate colitis) are listed in the following table:

\begin{tabular}{|l|l|l|l|l|l|l|}
\hline $\begin{array}{l}\text { Mutated } \\
\text { Gene }\end{array}$ & $\begin{array}{l}\text { UC, \% } \\
(\mathrm{n}=37)\end{array}$ & $\begin{array}{l}\mathrm{CD}, \% \\
(\mathrm{n}=16)\end{array}$ & p-value & $\begin{array}{l}\text { All IBD, \% } \\
(\mathrm{n}=56)\end{array}$ & $\begin{array}{l}\text { TCGA, \% } \\
(\mathrm{n}=224)\end{array}$ & p-value \\
\hline TP53 & 67.6 & 68.8 & 0.92 & 69.6 & 54.0 & 0.03 \\
\hline KRAS & 16.2 & 31.1 & 0.27 & 19.6 & 42.0 & $<0.01$ \\
\hline PIK3CA & 16.2 & 25.0 & 0.70 & 17.9 & 20.1 & 0.85 \\
\hline APC & 5.4 & 31.3 & 0.02 & 12.5 & 75.0 & $<0.01$ \\
\hline IDH1 & 5.4 & 12.5 & 0.58 & 7.1 & 1.3 & 0.03 \\
\hline SMAD4 & 10.8 & 0.0 & 0.30 & 7.1 & 11.6 & 0.33 \\
\hline FBXW7 & 8.1 & 0.0 & 0.55 & 8.9 & 16.5 & 0.16 \\
\hline
\end{tabular}

There was a trend towards increased PI3K pathway mutations in older patients $(\mathrm{OR}=1.07, \mathrm{p}=0.055)$.

Conclusions: Significant differences exist in the mutational landscape of IBD-associated and non-IBD-associated CRCs. There is a higher frequency of TP53 and IDHI mutations and a lower frequency of $A P C$ and $K R A S$ mutations in IBD-CRCs. The mutational profiles of CRCs in CD and UC also differ; CD CRCs are more likely to exhibit $A P C$ mutations, while SMAD4 and FBXW7 mutations may be specific to UC CRCs. 
630 Significance

Manju Ambelil, David Saulino, Atilla Ertan, Andrew W Dupont, Sushovan Guha, Mamoun Younes. University of Texas Health Science Center at Houston McGovern Medical School, Houston, TX.

Background: Recent studies examining immunohistochemical (IHC) staining of colorectal biopsies for CMV reported that some cases showed rare small positive nuclei that were called equivocal for CMV. The aim of this study was to determine the extent of equivocal IHC staining for CMV, and whether it is of clinical significance. Design: During a two-year period, 2014 and 2015, two hundred twenty-one cases of colon and rectal biopsies were stained for CMV by IHC. Slides were reviewed and staining results were recorded as negative when there was completely negative nuclear staining, "unequivocal" when at least one large "megalic" nucleus positively stained, and "equivocal" with only small positively stained nuclei without any positive large (megalic) nuclei. Clinical data were obtained from the medical records.

Results: A total of $83(38 \%)$ of the 221 cases showed nuclear staining in at least one cell. Fifty-two cases ( $24 \%$ of all tested, $63 \%$ of all positive case) showed equivocal staining. Of these equivocal cases, 4 had completely normal histology, and 9 showed mild nonspecific changes. In the pathology reports, unequivocal staining was reported to the clinicians as "rare" or "occasional" small nuclei positive for CMV by immunoperoxidase staining. Twenty-nine of the patients with equivocal biopsies were treated with antiviral drugs or discontinuation of immunosuppressive drug and of these $24(83 \%)$ had significant improvement or complete resolutions of the disease/diarrhea. By contrast, only 5 of $14(36 \%)$ patients with equivocal staining who did not receive specific treatment improved $(\mathrm{p}=0.002)$. Documentation on treatment and/or follow-up was not available for 9 patients with equivocal staining. One of the five patients with equivocal staining who did not respond to antiviral treatment had a flare up of IBD which was the reason for worsening of symptoms.

Conclusions: In summary, more patients tested for CMV by IHC show occasional positive small nuclei than classic megalic nuclei, including transplant recipients with normal or minimal nonspecific histology and chronic diarrhea. The majority of these patients respond well to specific antiviral treatment. Therefore 1) all colorectal biopsies from immunosuppressed patients, even with normal histology, should be tested for $\mathrm{CMV}$ by IHC and 2) rare small positive nuclei should be reported to the clinicians as positive for CMV.

631 Colonic Carcinomas with Sporadic Loss of Mismatch Repair Proteins Are Associated with Loss of HLA Class I

Kshitij S Arora, Theodoros Michelakos, Lei Cai, David T Ting, Cristina Ferrone, Soldano Ferrone, Vikram Deshpande. massachusetts General Hospital, Boston, MA. Background: The programmed death 1 (PD-1) pathway represses cytotoxic immune responses and protects tumors from the intratumoral T-cell infiltrate. Gastrointestinal neoplasms with defective mismatch-repair (MMR) status show dramatic and durable clinical benefit of immune checkpoint blockade with PD-1 antibodies, however, only half the MSI (microsatellite instability) positive colon carcinomas respond. Herein, we explore three escape mechanisms that may predict resistance to PD1 therapy: 1) absence of PDL1 expression, 2) absence of PD1 expression, 3) loss of tumor HLA class 1 proteins. We also validate the immunohistochemical (IHC) assay using a branch chain ISH assay for PD-L1.

Design: 96consecutive colonic adenocarcinomas without evidence of Lynch syndrome were evaluated to determine MSI status, PD-L1 reactivity, and HLA Class I antigen expression. MSI status was evaluated on an IHC platform using antibodies agains MLH1, PMS2, MSH2 and MSH6. IHC for PDL1 (clone E1L3N) and Class I HLA (clone HC10) was performed. In situ hybridization (ISH) for PD-L1 and PD1 (Affymetrix, CA) was performed. The results were validated using TCGA colon cancer dataset. Results: Neoplastic cells express Class I HLA at the advancing edge of the tumor Tumors with MSI loss were more likely to show loss of HLA class I (MSI loss $29 \%$ $(7 / 24)$, MSI intact $7 \%(5 / 72)(\mathrm{p}=.0 .004)$. PDL1 IHC was positive in $41 \%$ of cases and detected in either macrophages $(31 \%)$ or tumor cells $(9 \%)$. PD-L1 ISH correlated strongly with PD-L1 IHC (Pearson correlation 0.78); ISH signal was noted in either macrophages or tumor cells. Tumors with MMR protein loss were more often positive for PD-L1, in both macrophages and tumor cells $(\mathrm{p}=0.02)$; this correlation was validated on the TCGA cohort $(\mathrm{p}=0.0001) .21 \%$ of tumors with loss of MMR proteins expressed PDL1 in tumor cells compared to $5 \%$ in the MSI intact cohort. PD1 positive lymphocytes $(>10 / \mathrm{HPF})$ correlated with positive PDL1 staining in neoplastic cells $(\mathrm{p}=0.03)$, but not with PDL1 staining in macrophages $(\mathrm{p}=0.18)$. All tumors with $\mathrm{PD}-\mathrm{L} 1$ reactivity in neoplastic cells were also positive for PD1 lymphocytes.

Conclusions: The high rate of loss of HLA class 1 in tumors with sporadic loss of MMR proteins represents a common escape mechanism in this class of tumors and may predict resistance to immunotherapy. IHC analysis of PD-L1 and class I HLA may assist in tailoring checkpoint inhibitor therapy in colonic carcinomas.

632 Albumin RNA In Situ Hybridization in Hepatocellular Carcinomas and Other Neoplastic and Non-Neoplastic Tissue: Can This Be a Clinically Useful Marker?

Vaidehi Avadhani, Momin T Siddiqui, Diane Lawson, Cynthia Cohen. Emory University, Atlanta, GA.

Background: RNAscope is an RNA in situ hybridization (RISH) technology with a unique signal amplification and background suppression strategy. Albumin is a well-known sensitive and specific marker for hepatocellular carcinoma (HCC) but immunohistochemical detection has proven to be difficult practically. With the current use of RISH, we evaluated the efficacy of albumin ISH as a marker of HCC and compared its use in other neoplastic and normal tissues.
Design: We evaluated 22 hepatocellular carcinomas (21 whole sections and 1 core in a tissue microarray [TMA]), and neoplastic (12) and non-neoplastic tissue (9) in a TMA with 2 cores each (as shown in Table 1). We performed RISH for albumin using the RNAscope detection kit (Leica Biosystems, Buffalo Grove, IL) with the Bond III autostainer, using probe Hs-ALB-01 (Advanced Cell Diagnostics, Newark, CA). A negative probe, Dap B, and a positive probe for RNA (PP1B) were also included. Characteristic dot like cytoplasmic reactivity was considered positive. A semiquantitative method of scoring was used: $0=$ no staining at all; $1=<5 \%$ tumor cells; $2=5-50 \%$ tumor cells and $3=>50 \%$ tumor cells.

Results: Albumin RISH was diffusely positive (3+) in 22 of $22 \mathrm{HCCs}$ (figure 1) and 1 neonatal liver.

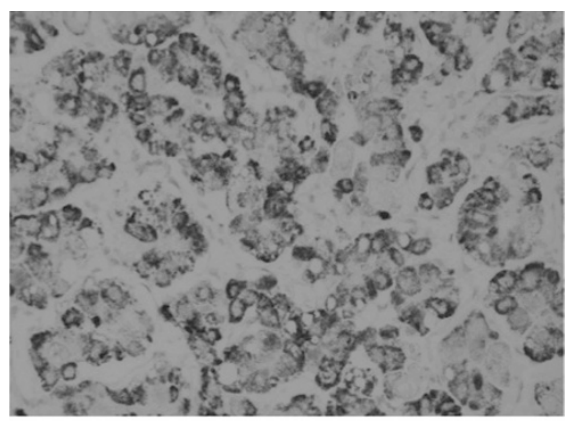

Albumin ISH positive in $\mathrm{HCC}(20 \mathrm{X})$

\begin{tabular}{|l|c|}
\hline \multicolumn{1}{|c|}{ Table 1. Albumin RISH } & Albumin RISH Stain Result \\
\hline Tonsil & - \\
\hline Normal Breast & - \\
\hline Placenta & - \\
\hline Prostate & - \\
\hline Colon & - \\
\hline Skin & - \\
\hline Spleen & - \\
\hline Salivary gland & - \\
\hline Neonatal Liver & + \\
\hline Breast Carcinoma & - \\
\hline Renal Cell Carcinoma & - \\
\hline Medullary Thyroid Carcinoma & - \\
\hline Melanoma & - \\
\hline Pancreatic NET & - \\
\hline Ovarian Carcinoma & - \\
\hline Mesothelioma & - \\
\hline Leydig Cell Tumor & - \\
\hline Thymoma & - \\
\hline HCC & + \\
\hline Papillary Thyroid Carcinoma & - \\
\hline Colon Carcinoma & - \\
\hline
\end{tabular}

All the other neoplastic $(12,100 \%)$ and non-neoplastic $(9,100 \%)$ were negative. 10 of $22 \mathrm{HCCs}$ were poorly differentiated carcinomas, all of which were positive for Albumin RISH.

Conclusions: In our cohort, Albumin RISH showed a sensitivity of $100 \%$ and specificity of $100 \%$. Albumin RISH appears to be a useful marker, also in poorly differentiated HCCs, when other hepatocellular markers may be negative.

633 Peritumoral Lymphoid Cuff in Gastrointestinal Schwannomas Is Significantly Correlated with Regional Lymph Node Enlargement: A Study of 118 Consecutive Cases from a Single Institute

Hyunsik Bae, Ha Young Park, Jinah Chu, Michael Van Vrancken, Kyoung-Mee Kim. Samsung Medical Center, Sungkyunkwan University School of Medicine, Seoul, Republic of Korea; Tulane University School of Medicine, New Orleans, LA.

Background: Primary schwannomas of the gastrointestinal (GI) tract are uncommon subepithelial lesions that usually follow a benign course, although rare cases of malignant transformation have also been reported. The distinction between schwannomas and gastrointestinal stromal tumors (GIST), another subepithelial GI tract tumor, is an important aspect in the preoperative planning of these lesions. One helpful distinction that has been reported is that regional lymphadenopathy (LAD) is more often associated with schwannomas than with GISTs.

Design: The Samsung Medical Center pathology database was queried for all resected gastrointestinal schwannomas from January 1998 to June 2016. A total of 118 cases were found.

Results: Locations of the lesions varied and included 85 gastric (72\%), 11 colonic (9.3\%), 7 esophageal $(5.9 \%), 3$ pancreatic $(2.5 \%), 1$ hepatic $(0.8 \%)$, and 11 peritoneal $(9.3 \%)$. The female to male ratio was $1.7: 1$, and ages ranged from 25 to 80 (mean 55.5) years. The size of the tumors ranged from 0.2 to $11 \mathrm{~cm}$ with a mean of $3.8 \mathrm{~cm}$. None of GI schwannomas recurred or showed any evidence of malignant transformation. Histologically, in the majority of cases ( 75 cases $-63.6 \%$ ), a peripheral lymphoid cuff 
was noted around the schwannoma ranging in thickness from 0.5 to $6 \mathrm{~mm}$ (mean 2.8 $\mathrm{mm}$ ). Regional LAD was assessed through review of prior radiographic and concurrent pathologic findings. Of the 106 cases for which data was available for, 48 cases $(45.3 \%)$ showed regional LAD. The presence of the lymphoid cuff and regional LAD showed significant positive correlation $(p=0.002)$.

Conclusions: Within the GI tract, schwannomas are a rare entity which need to be distinguished from GISTs. The presence of regional LAD favors schwannoma. Histologically, a peripheral lymphoid cuff is seen in the majority of GI tract schwannomas and positively correlates with the presence of regional LAD. However, in a significant subset $(36.4 \%)$, no lymphoid cuff is present magnifying the importance of correlating preoperative radiologic studies and postoperative pathologic diagnoses in the distinction between GI tract schwannomas and GISTs.

634 Prognostic Significance of Tumour Regression Grade at Primary Site and Its Correlation with Clinical and Histological Parameters in Post Chemotherapy Excision Specimens of Oesophagus-Single Institutional Study

Ganesh Bahirwade, Shubhada Kane, Rajiv Kumar, Swapnil Rane, Anuj Verma, Kumar Prabhash, Pramesh Shanmugham. Tata Memorial Hospital, Mumbai, India.

Background: Carcinoma of oesophagus is one of the most lethal malignancies. Surgery is the main treatment modality. Neoadjuvant chemotherapy helps to reduce the tumour burden and increases survival when followed by surgery. Histological assessment of tumour response-tumour regression grade(TRG), its prognostic significance and correlation with clinical and histological parameters is not extensively studied in cases of carcinoma oesophagus. This study focuses on correlation of TRG with clinical, histological parameters and survival (DFS/OS.)

Design: One hundred forty cases of post NACT excision specimens of oesophagus during the year 2014 were retrieved from hospital registry. Follow up details were obtained from electronic medical record. Histology sections were reviewed, TRG was assigned in each case using Mandard grading system. Clinical and histological parameters such as tumour size, site of involvement, histologic type, pTNM stage, circumferential resection margin(CRM), tumour grade and survival were correlated with TRG. Statistical analysis was performed with Kaplan Meier test, log- rank test and Chi square test.

Results: Distribution of TRG $1-5$ was as follows: TRG $1-26.4 \%, 2-10 \%, 3-10 \%$, $4-22.5 \%$ and $5-31.5 \%$, TRG was significantly correlated with pre- chemotherapy size (largest dimension) of the tumour, site of involvement, histologic type, pTstage, pN stage and CRM status. TRG was significantly correlated with DFS but not with OS. Conclusions: This study confirms the importance of grading tumor regression in the survival of patients with esophageal carcinoma with neoadjuvant chemotherapy. It also suggests that TRG should be evaluated in post NACT oesophagectomy specimen.

635 Unique Differential Expression of PD-L1 in Anal Squamous Cell Dysplasia and Invasive Carcinoma

Andrew Bandy, Xiaoming You, Haonan Li, Jie Liao, Sambasiva Rao, Guang-Yu Yang Northwestern University, Chicago, IL.

Background: Escape from immune surveillance is crucial in tumor development for avoiding death via upregulation of programmed death ligand 1 (PD-L1) on their cell surface and becoming functionally anergic to the immune system. Recently much research has focused on the expression level of PD-L1 on tumor cells and targeting PD-L1 with immunotherapy which overcomes this stealth mechanism and boosts the immune response. With limited data regarding PD-L1 status in patients with anal squamous cell carcinoma (SCC), it is worth determining whether PD-L1 is expressed in HPV-associated anal SCC since HPV-positive head and neck SCC have higher PDL1 expression and benefit from anti PD-L1 drugs, and whether there is a difference in PD-L1 expression in HPV-associated dysplasia compared to SCC.

Design: A total of 42 patients with either in situ and/or invasive anal SCC from 37 biopsies and 5 resections were collected. Immunohistochemistry for p53, p16 and PDL1 was performed for all cases with proper positive and negative controls. Positive staining was evaluated based on staining intensity in either the membrane, cytoplasm or nuclei of tumor cells (negative, weak, moderate and strong) and the proportion of overall tumor that stained ( $\%$ positive cells).

Results: In this cohort, All 42 cases (100\%) displayed HPV-associated diffuse or intense cytoplasmic/nuclear positive p16 staining in the high grade dysplasia/in situ and invasive SCC, and all cases exhibited scattered p 53 positive cells (wild-type p53 protein). For PD-L1, 17/31 (55\%) cases displayed at least moderate staining in tumor cells in the invasive component of anal SCC (Fig A), with a similar staining profile seen in $7 / 30(23 \%)$ cases of high-grade dysplasia (Fig B). All background normal squamous mucosa in 14 cases was negative for expression of PD-L1 (Fig C).

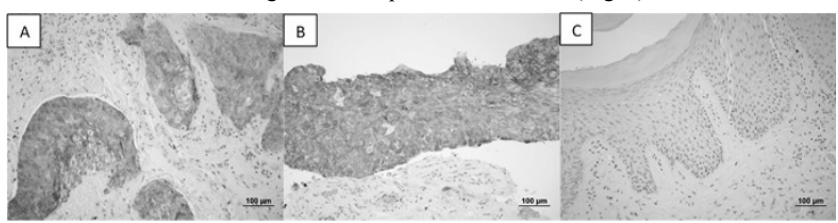

Conclusions: Our study demonstrated that PD-L1 is upregulated in anal HPV-associated invasive SCC and high-grade dysplasia compared to non-dysplastic squamous mucosa Additionally, the high frequency of PD-L1 expression in invasive SCC suggests PD-L1 may serve as a potential biomarker to predict progression from in situ SCC to invasive SCC. Our data imply a potential benefit for anal SCC patients with IHC expression of PD-L1 by incorporating immunotherapy into their treatment regimen.
636 RICTOR Overexpression/Amplification in Advanced Solid Tumors; a Correlation Study Utilizing Immunohistochemistry, Fluorescence In Situ Hybridization, and Targeted Sequencing

Heejin Bang, Soomin Ahn, Jeeyun Lee, Seung Tae Kim, Michael Van Vrancken, Ha Young Park, Kyoung-Mee Kim. Samsung Medical Center, Sungkyunkwan University School of Medicine, Seoul, Korea; Ewha Womans University School of Medicine, Seoul, Korea; Tulane University School of Medicine, New Orleans, LA; College of Medicine, Inje University, Busan Paik Hospital, Busan, Korea.

Background: RICTOR is a key component of the mTOR complex 2 (mTORC2). When activated, this complex promotes cellular proliferation and survival through

the activation of downstream AGC kinase family members. Amplification of RICTOR serves as a promising emerging actionable genomic alteration for which targeted therapy will likely be developed. However, the incidence of RICTOR amplification in advanced solid tumors is an area that has not been fully explored.

Design: In this study, a multimodal design was used in order to study RICTOR amplification through the use of immunohistochemistry (IHC; $n=416$ ), fluorescence in situ hybridization (FISH; $n=51)$ and deep targeted sequencing $(n=51)$ in a number of solid organ tumors including colorectal $(n=193)$, gastric $(n=86)$, renal cell carcinoma $(n=34)$ and other solid cancers $(n=103)$. These cases were previously also enrolled for NEXT1 and NEXT2 trials. For IHC, cases were considered positive if there was +1 , +2 , or +3 staining. Blush weak to negative staining was considered negative. For FISH, a RICTOR/CEP17 ratio $>2.0$ was defined to be amplified. For targeted sequencing, an estimated copy number $\geq 4$ was considered amplified.

Results: RICTOR was overexpressed in 202 cases $(48.6 \% ; 1+, 30.3 \% ; 2+, 14.4 \%$, $3+, 4.1 \%$ ) by IHC, and amplified in 29 cases $(56.9 \%)$ by FISH and 24 cases $(47.1 \%)$ by targeted sequencing. Overexpression/amplification of RICTOR was found in 111 (57.5\%) colorectal cancers, $38(44.2 \%)$ gastric cancers, $16(47.1 \%)$ renal cell carcinomas, and $37(35.9 \%)$ other tumors. The IHC results were significantly positively correlated with FISH $(\mathrm{p}<0.0001 ; \mathrm{r}=0.66)$, but no significant correlation was seen between IHC and targeted sequencing $(\mathrm{p}=0.49)$.

Conclusions: In conclusion, RICTOR overexpression/amplification is fairly common in a variety of advanced solid tumors. Additionally, IHC for RICTOR appears to be a reliable surrogate marker for amplification, which may very well have future therapeutic implications.

637 Low Grade Appendiceal Mucinous Neoplasms with Neoplastic Epithelium Confined to the Appendix: 45 Cases with Clinical Follow-Up Brad D Barrows, Deepti Dhall. Cedars Sinai Medical Center, Los Angeles, CA.

Background: Appendiceal mucinous neoplasms continue to be a challenge for pathologic classification and clinical management. The World Health Organization (WHO) divides these tumors into three categories: adenoma (low grade dysplastic mucinous epithelium with intact muscularis mucosa), low grade appendiceal mucinous neoplasm (LAMN) (mucin and/or dysplastic epithelium beyond the muscularis mucosa, including extra-appendiceal low grade neoplastic epithelium and/or mucin), and mucinous adenocarcinoma (infiltrative invasion). The anxiety concerning a diagnosis of LAMN in routine clinical practice can be at least partly attributed to the limited number of outcome studies to guide management. This single institutional study will focus on the clinical behavior of 2 subgroups of LAMN: mucin pools and neoplastic epithelium confined by appendiceal serosa and cases with acellular mucin alone beyond the appendiceal serosa.

Design: 104 appendiceal mucinous neoplasms (entirely submitted for histologic examination) were retrieved from the Pathology database $(1995-2016) .45$ cases met our inclusion criteria (extended follow-up defined as $>6$ months and absence of neoplastic epithelium outside the appendiceal serosa).

Results: Two subgroups of LAMN were identified: 36 cases with mucin and neoplastic epithelium confined by appendiceal serosa and 9 cases with extra-appendiceal acellular mucin. All were managed by either appendectomy ( $\mathrm{n}=34 ; 4$ with partial cecectomy) or partial colectomy $(n=11)$. No tumor recurrence was noted in either group at a mean follow-up of 54 months (median 50 months, range 7-146). Interestingly, all 4 appendectomies with positive proximal margin had no residual lesion in subsequent hemicolectomy specimens. However, one patient was noted to have a single focus of acellular mucin removed as an omental nodule at the time of colectomy, and this patient has been disease free through 74 months of follow-up.

Conclusions: Our study adds to the literature supporting an excellent prognosis with negligible risk of recurrence in cases of LAMN confined within the appendiceal serosa These tumors may be best treated (or even cured) by appendectomy alone. Right hemicolectomy may not be necessary even in cases noted to have low grade mucinous dysplasia at margin. Regular follow-up with colonoscopy including observation of the appendix resection site may be a more efficient method of recurrent disease monitoring.

638 Tract Cancers

Prashant Bavi, David Hedley, Jennifer J Knox, Stefano Serra. University Health Network, University of Toronto, Toronto, ON, Canada; Clinical Oncology, University Health Network, University of Toronto, Toronto, ON, Canada.

Background: Indoleamine 2,3-dioxygenase 1 (IDO1) is one of the key rate limiting enzymes involved in tryptophan metabolism through the kynurenine pathway. IDO1 has been shown to exert an immunosuppressive effect in the tumor microenvironment and prevent exaggerated T cell response. Here, we investigate IDO1 as a potential prognostic marker in biliary tract cancerand correlate its expression with clinicpathological variables.

Design: We investigated IDO1 expression by immunohistochemistry in a wellcharacterized tissue microarray cohort of 161 biliary tract cancer patients. Anti- hIDO 
$\mathrm{mAb}$ (c14.16H1), a purified 4.16H1 anti-IDO1 antibody, Ludwig Institute for Cancer Research Ltd., was used. IDO1 expression was grouped into present and absent based on cytoplasmic and/or nuclear expression observed in the tumor and the stromal cells. IDO1 expression was correlated with tumor pathological features including TNM staging as well as clinical outcomes such as overall survival.

Results: IDO1 expression in the tumor was observed in $34.7 \%$ (56/161) of stage I-IV biliary tract cancers. IDO1 expression correlated with tumor $\operatorname{size}(\mathrm{p}=0.00251$; $<3 \mathrm{~cm}$ ), perineural invasion $(\mathrm{p}=0.0173)$, lack of tumor vein invasion $(\mathrm{p}=0.0191)$, and site ( $\mathrm{p}=0.0099 ; 56 \%$ of bile duct cancers). IDO1 expression was not associated with age, Stage, histology and lymph node involvement. No differences in overall survival $(\mathrm{p}=0.2287)$ or recurrence free survival were observed in tumors with and without IDO1 expression. However, biliary tract cancers from hilar site with IDO1 expression showed a poor overall survival $(\mathrm{p}=0.0317)$ as compared to those with no IDO1 expression.

Conclusions: IDO1 expression was observed in a significant subset of biliary tract carcinomas. Our results highlight subgroups of biliary tract cancer patients from hilar site with IDO1 expression are more aggressive, and further studies in a larger cohort could act as an adjunct to decide on IDO1 adjuvant treatment strategies in these patients.

\section{Spectrum of Histopathological Changes Induced by PD-1/PD-L1} Blockade in GI Biopsies: A Preliminary Study

Prashant Bavi, Stefano Serra, Marcus Butler, Runjan Chetty. University Health Network, Toronto, ON, Canada; Princess Margaret Cancer Centre, University Health Network, Toronto, ON, Canada.

Background: Immunotherapy targeting programmed cell death protein 1 and its ligand (PD-1/PD-L1) have dramatically improved outcome in multiple tumors. There is a paucity of data on the histopathologic features of PD-1/PD-L1 inhibitors (PDIs) induced immune related adverse events (irAEs) in the GI tract.

Design: We identified 144 patients on combination clinical trials with PDI (2011-2016) from the Tumor Immunotherapy Program database at The Princess Margaret Cancer Centre. Of these 18 patients underwent GI endoscopy and biopsy for symptoms clinically attributed to irAEs. Patient electronic medical records were reviewed for type of primary tumor, start and stop dates of therapy, types of therapy, endoscopy findings and history of autoimmune disorders. Histopathologic changes such as architecture, surface ulceration, cryptitis, crypt abscesses, apoptosis of basal crypt, dilated damaged crypts, expansion of lamina propria, lymphoplasmacytic infiltrate, granulomas, lymphocytic colitis, microscopic colitis were studied. Immune infiltrate was characterized in the intraglandular compartment and lamina propria using a IHC panel of CD3, CD8, CD4, FoxP3, CD20 and CD4.

Results: There were 11 men and 7 women with an age range of 24-74 years. The study cohort consisted of 36 GI biopsies: large intestine $(n=24)$; small intestine $(n=5)$; stomach $(\mathrm{n}=5)$ and others ( $\mathrm{n}=2$; pancreas and esophagus). Colonoscopy findings ranged from normal to colitis to erythematous friable and ulcerated mucosa. Patients with symptomatic clinical PDI-induced colitis did not show any significant histological changes in most of the intestinal biopsies (18 biopsies from 7 patients). However, striking changes reflecting a mixture of inflammatory bowel disease, drug induced toxicity and graft versus host disease changes were noted in only 10 biopsies from 3 patients. The mean $\mathrm{CD} 3(\mathrm{p}=0.0019)$ and $\mathrm{CD} 8(\mathrm{p}=0.0019)$ counts in the intraglandular compartment were significantly higher in the PDI treated group as compared to the control group with IBD.

Conclusions: No specific histological features were identified that could distinguish PDI induced colitis and irAEs from other colitis. However, presence of multiplicity of histological patterns in the same biopsy and a significant increase in intraglandular $\mathrm{CD} 3$ and CD8 lymphocytes were noted. For correct diagnosis of such type of colitis, awareness of irAEs and treatment history will be the cornerstone of diagnosis

\section{Prognostic Value of the Nestin Staining in Human Pancreatic}

\section{Ductal Adenocarcinoma}

Mohadese Behtaj, Wei Xin. University Hospitals Cleveland Medical Center/Case Western Reserve University, Cleveland, OH.

Background: Nestin, a class VI intermediate filament protein, detected in neural stem cells during development. The expression of nestin has been detected in various human malignancies. Multiple study showed nestin is a stem cell marker of pancreatic ductal adenocarcinoma (PDAC) in cell lines. The primary aim of this study is to investigate nestin expression in human PDACs and a possible prognostic marker.

Design: 175 cases of PDAC were studied by tissue microarray (TMA), ranging from 29-85 year old, and F/M: 94/81.Immunostain of Nestin was performed in the hospital diagnostic lab; the staining was scored and correlation with clinical findings was evaluated.

Results: Among the 175 cases, only 23\% (41/175) showed detectable nestin expression.

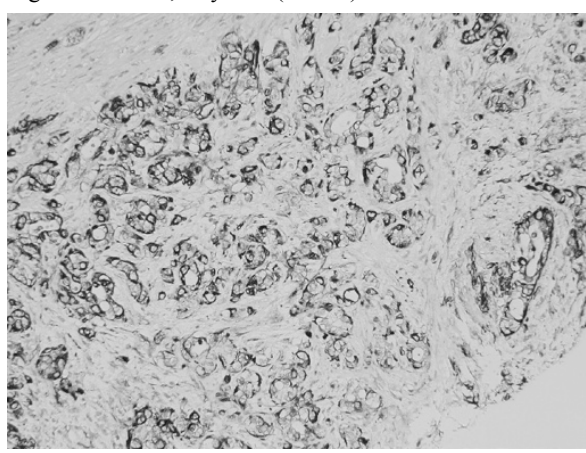

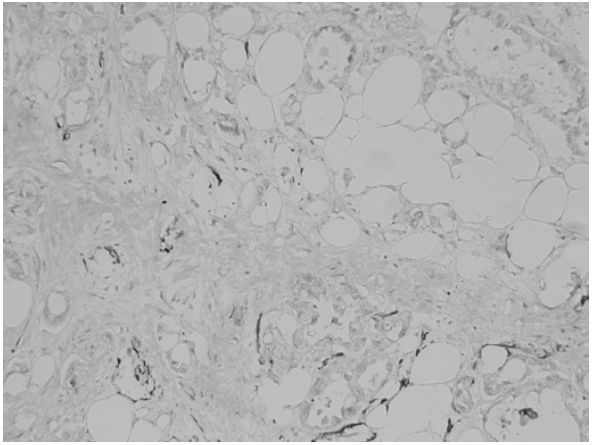

There is no association among Nestin expression, tumor grade, lymph node status, angiolymphatic invasion and perineural invasion.

Conclusions: Nestin is considered as a stem cell marker which showed prognostic value in animal and cell lines studies. Our study showed that Nestin expression can be detected in a subset of PDACs, and not associated with common prognostic markers.

\section{TTF-1 Expression Is Upregulated in the Corpus in Autoimmune} Atrophic Gastritis

Ava Bhattarai, Andrew M Bellizzi. University of Iowa, Iowa City, IA.

Background: We routinely perform a panel of site of origin immunohistochemistry (IHC), including occasionally TTF-1, in metastatic neuroendocrine tumors (NETs) of occult origin. Upon accidentally staining a gastric NET arising in autoimmune metaplastic atrophic gastritis (AMAG), we discovered strong TTF-1 expression in the background mucosa. As AMAG is characterized by intestinal and pancreatic acinar metaplasia, we speculated that at least a portion of what is referred to as pyloric metaplasia in this setting may in fact be bronchopulmonary metaplasia.

Design: TTF-1 IHC was performed in 33 AMAG (22W:11M; mean age 59; 48 corpus, 24 antral biopsies), 43 Helicobacter pylori (HP) gastritis (20W:23M; mean age 43; 50 biopsies), and 19 non-gastritis patients ( 9 full-thickness sections from sleeve gastrectomies, 10 normal corpus biopsies). HP biopsies were assessed for gastritis topography: corpus-predominant $(\mathrm{CP})$, pangastritis $(\mathrm{PG})$, antral-predominant (AP). We also stained other pyloric metaplasias (20 chronic carditis, 13 chronic ileitis, 22 chronic cholecystitis) and tubal gut adenocarcinomas (18 esophagus, 36 stomach, 23 small intestine, 46 colon). For biopsies, TTF-1 was quantified as mean number of TTF-1-positve glands/biopsy fragment. Two-sided Fisher's exact, Mann-Whitney, and Kruskal-Wallis tests were used ( $\mathrm{p}<0.05$ significant).

Results: TTF-1-positivity was seen in 40/48 (83\%) corpus and only $3 / 24(13 \%)$ antral biopsies from 29/33 (88\%) AMAG patients; 14/50 (28\%) biopsies from 14/43 (33\%) HP gastritis patients; and 7/9 (78\%) sleeve gastrectomy sections and 4/10 (40\%) normal corpus biopsies. The mean (median; range) number of TTF-1-positive glands/fragment was $8.5(4.5 ; 0-42)$ for AMAG corpus, $\mathbf{0 . 4 6}(0 ; 0-5.5)$ for HP gastritis, and $\mathbf{0 . 2 0}(0$ $0-1)$ for normal corpus biopsies. TTF-1 was not expressed in 20 chronic carditis and 13 chronic ileitis biopsies or in 123 tubal gut adenocarcinomas; $1 / 22(5 \%)$ chronic cholecystitis cases was focally positive. On a per patient and per biopsy basis, the frequency and extent of TTF-1-positivity was significantly greater in AMAG than HP gastritis (all $p<0.0001)$. Extent of TTF-1-positivity did not significantly differ between HP gastritis and normal $(\mathrm{p}=1)$, and HP gastritis topography did not reach statistical significance for frequency $(\mathrm{p}=0.054)$ or extent $(\mathrm{p}=0.23)$ of positivity. Thresholds of $\geq 2$ and $\geq 3$ TTF-1-positive glands/fragment were $70 \%$ sensitive, $91 \%$ specific and $58 \%$ sensitive, $98 \%$ specific for a diagnosis of AMAG (vs. HP gastritis).

Conclusions: TTF-1 is significantly overexpressed in atrophic gastritis, a finding that is both biologically intriguing and potentially diagnostically useful.

\section{Chromosome 17 Copy Number Instability Is Associated} with Favorable Prognosis in Non-Surgically Treated Gastroesophageal Adenocarinoma but May Impair Response to Trastuzumab

Jacqueline Birkness, Neal Spada, Sanja Dacic, James D Luketich, Katie S Nason, Weijing Sun, Jon Davison. Johns Hopkins University, Baltimore, MD; University of Pittsburgh, Pittsburgh, PA.

Background: Chromosomal instability (CIN), defined as variation in chromosome copy number, has been shown to result in unstable aneuploidy. In esophageal adenocarcinoma, chromosome 17 (Chr17) aneusomy is associated with heterogeneous HER2 amplification, a marker of poor prognosis [PMID: 22987085], but the association between Chr 17 CIN (CIN17), Chr17 aneusomy and response to trastuzumab has not been studied.

Design: We retrospectively analyzed individual cell Chr17 centromere counts $(\geq 60$ cells per tumor) in 348 gastroesophageal adenocarcinomas that were tested for HER2 amplification by FISH. As an estimate of CIN17, we calculated the percentage of cells with centromere counts differing from the mode (modal centromere deviation, MCD) [PMID: 21784954]. We analyzed the association of CIN17 with Chr17 aneusomy, HER2 amplification (HER2/CEP17>2.0), histologic type and clinical outcome.

Results: We found $45 \%(158 / 348)$ of tumors were positive for CIN17 (MCD $\geq 30 \%)$ and $28 \%$ (99/348) positive for extreme CIN17 (MCD $>45 \%$ ). CIN17 positive tumors were more likely to be polysomic and Lauren intestinal type, but there was no association with between CIN17 and HER2 amplification (figure 1A, B, C). In patients who received primary chemotherapy $(n=77)$, there was a $58 \%$ reduction in overall mortality associated with extreme CIN17 (HR=0.42, 0.25-0.72, P=0.002) independent of age, stage and addition of trastuzumab. Addition of trastuzumab to chemotherapy showed a 
trend toward prolonged survival compared to chemotherapy alone only in the subgroup without extreme $\mathrm{CIN}(\mathrm{P}=0.054)$. In patients treated by surgery, $\mathrm{CIN} 17$ was not associated with differences in survival.
A

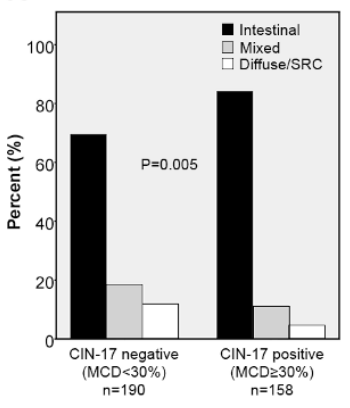

C

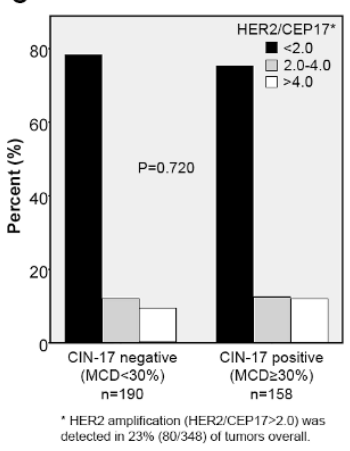

B

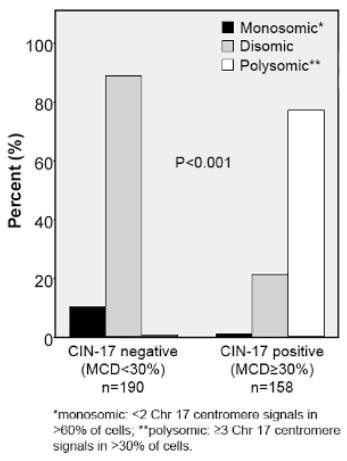

Conclusions: CIN can be quantified from routine centromeric FISH data. Extreme CIN17 was a favorable prognostic factor after primary pharmacologic therapy, but could impair response to trastuzumab.

\section{3}

ErbB3 Overexpression Predicts Survival in Primary Colorectal

Cancer

Alina Bocicariu, Kevin Yi Mi Ren, Ravi Ramjeesingh, Nazik Hammad, James J Biagi, Christopher Nicol, Lois Mulligan, Harriet Feilotter, David Hurlbut, Scott Davey. Queen's University, Kingston, ON, Canada; Dalhousie University, Halifax, NS, Canada. Background: ErbB3 is a member of epidermal growth factor receptor (EGFR) family and has dual roles in promoting cell proliferation and preventing apoptosis through activation of PI3K/AKT pathway. ErbB3 as a heterodimerization partner also promotes tumor invasion and metastasis in many solid tumors. ErbB3 expression and its correlation with the clinical outcome in primary colorectal cancer (CRC) have been assessed in a limited number of studies with contradictory results. We examined ErbB3 expression in a large cohort of primary $\mathrm{CRC}$ and assessed its performance as a potential independent prognostic marker.

Design: ErbB3 expression was assessed by immunohistochemistry using tissue microarrays from 128 cases of primary CRC patients (stage I-IV) treated in our institution. Intensity and extent of ErbB3 staining were independently assessed by 2 pathologists on a scale from 0 to 3 . Cytoplasmic (c) and membranous (m) erbB3 staining scores were calculated as a weighted average from 3 core samples per tumour. Univariate analysis of average scores and clinicopathological outcome measures from patient chart review was completed.

Results: ErbB3 is overexpressed in CRC compared to normal colonic epithelium $(\mathrm{n}=119 ; \mathrm{p}=0.01)$. The proportion of positive lymph nodes per case was positively associated with higher membranous and cytoplasmic erbB3 expression level in tumour cells $(n=125 ; \mathrm{p}<0.01)$. Both cytoplasmic and membranous ErbB3 overexpression in tumor cells were associated with improved 2 -year survival $(n=128 ; \mathrm{p}=0.02$ and $\mathrm{p}=0.03$, respectively), but were not associated with conventional prognostic factors such as tumor stage, lymph node involvement and lymphovascular invasion.

Conclusions: Our findings show that ErbB3 overexpression in primary CRC is associated with better 2-year survival. Given the divergent results of the role of ErB3 expression in primary CRC reported in the literature, standardizing ErbB3 expression analysis would be an important next step in evaluating the role of ErbB3 in CRC prognosis. Increased expression of ErbB3 at the protein level in particular merits further study as a possible prognostic indicator in colorectal cancer.

\section{Myosin 1e Expression Is Associated with Disease Progression} in Colorectal Cancer

Filippo Borri, Kodie Stem, Alessandro Bombonati, David Zuzga. Einstein Medical Center, Philadelphia, PA; La Salle University, Philadelphia, PA.

Background: Colorectal cancer (CRC) is the second leading cause of cancer-related death globally. The current histopathological staging paradigm for identifying high-risk patients and predicting chemotherapy benefit is imperfect. As a result, many patients are undertreated, putting them at increased risk for disease relapse, or overtreated, exposing them to unnecessary and harmful chemotherapy with no potential benefit.
Thus defining prognostic biomarkers to more accurately determine each person's risk of relapse and need for chemotherapy is a priority in colorectal cancer research. Myosin 1e (MYO1E) a long-tailed, class I myosin, regulates endocytosis, adhesion, migration, and invadosomes in kidney podocytes. Despite MYO1E expression in numerous cancers, its association with tumor progression is poorly understood.

Design: MYO1E was examined by IHC in CRC and matched normal adjacent tissue (NAT). A tissue microarray constructed from 119 CRC patients with available formalin fixed paraffin embedded tumor and NAT blocks and clinical annotation and included duplicate cores from each tissue block. Semiquantitative MYO1E immunohistochemical $\mathrm{H}$-scoring was conducted by blinded surgical pathologists. Next, the ratio of MYO1E tumor/NAT expression was calculated for each patient with both intact tumor and matched NAT tissue

Results: The ratio of MYO1E tumor/NAT expression was negatively associated with stage progression and increased invasiveness of tumors characterized by T-score. Moreover, tumors that had spread to regional lymph nodes had a significantly lower Myole expression ratio than tumors localized at the primary site. These data provide clinical evidence that reduced expression of Myo1E, compared to matched NAT, is associated with disease progression.
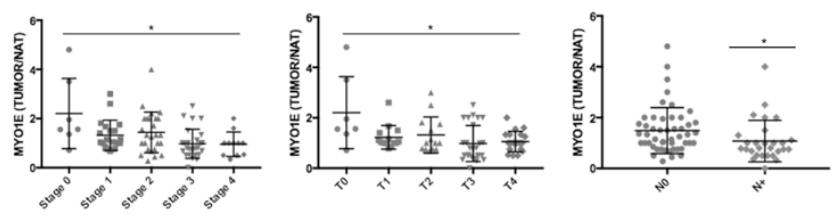

Conclusions: This study identifies MYO1E as a potential prognostic biomarker in CRC. Further examination of MYO1E expression to predict disease relapse and survival in a large retrospective study of CRC patients is suggested to define its prognostic utility to improve the clinical management of CRC patients.

\section{Incidental Gallbladder Carcinoma Associated with Dysplasia} and Acute Cholecystitis

$D E$ Bosch, M M Yeh, R A Schmidt, P E Swanson, C D Truong. U of Washington, Seattle, WA.

Background: Gallbladder carcinoma (GC) is an uncommon malignancy with an overall 5-year survival of less than 5\%. Due to overlap of clinical presentation with the more common cholecystitis, it is estimated $50-65 \%$ of all GCs are found incidentally. Epithelial dysplasia is identified in $\sim 50 \%$ of specimens with invasive carcinoma Recent expert panel guidelines have recommended histologic examination of the entire gallbladder in cases where initial sampling reveals dysplasia. Acute cholecystitis is frequently associated with GC and may complicate interpretation of epithelial atypia; however, its relationship to patient outcomes is unknown.

Design: We searched cholecystectomies with either dysplasia or GC within the last 15 years at our institution. For cases reporting additional sectioning after initial histologic evaluation, slides were reviewed. Gallbladder imaging reports were reviewed for all gallbladder carcinoma cases, when available. Patient demographic, clinical, and survival information was gathered from the medical record and publicly available databases. Results: 89 cases of GC, 34 high grade dysplasia (HGD), and 60 low grade dysplasia (LGD) were identified. Pre-operative imaging (either ultrasound or CT) only identified $52 \%$ of mass lesions in GC cases. Distant metastases were identified by post-operative imaging in $44 \%$ of cases. Among gallbladder specimens with epithelial dysplasia only at initial sampling, additional sectioning was performed and reported in $59 \%$ of HGD and $55 \%$ of LGD. Invasive carcinoma was identified after additional sectioning in 3 of 20 HGD (15\%) and 0 of 28 LGD cases. In multivariate analysis, significant correlations with decreased survival were found for specimens with associated acute cholecystitis (HR 6.5, $\mathrm{p}=0.001$ ), positive liver bed or cystic duct margins (HR 3.4, $\mathrm{p}=0.008$ ), and distant metastases (HR 2.2, p=0.05).

Conclusions: A high percentage of GCs are identified incidentally on routine histologic examination of gallbladder specimens, in part due to low sensitivity of pre-operative imaging. Additional sectioning of gallbladder specimens with HGD has a higher yield $(15 \%)$ for identifying invasive carcinoma than those with LGD $(0 \%)$. In our cohort, positive margin status and associated acute cholecystitis were significantly associated with reduced survival.

646 PD-L1 and PD-1 Expression Profile Depending on the Microsatellite Status and the Histological Subtype in Colorectal Carcinomas

Céline Bossard, Eva Ott, Delphine Dansette, Adrien Ouairy, Anne Jarry, Stéphane Bezieau, Claire Toquet, Jean-François Mosnier. CHU Hôtel Dieu, Nantes, France; Faculté de Médecine, Nantes, France.

Background: PD-1/PD-L1 blockade showed therapeutic efficacy in only microsatellite (MSI) colorectal carcinomas (CRC), however, the profile of PD-L1 and PD-1 expression in CRC is only partially described.

Design: We thus analyzed on FFPE whole-tissue sections of $80 \mathrm{CRC}$, the expression profile of PD-L1 by tumor and/or immune cells by immunohistochemistry (clone E1L3N) depending on the MSI status and the histological subtype, and correlated to the density of PD-1+ and Tbet+ (able to secrete IFNg known to induce PD-L1) tumorinfiltrating lymphocytes (TIL).

Results: $78 \%$ of MSI CRC (32/41) overexpressed PD-L1 either by tumor or immune cells versus $46 \%$ of MSS CRC (18/39) ( $\mathrm{p} 0.005)$. This overexpression was heterogeneous within the same tumor in most of cases. Among MSI CRC, PD-L1 was preferentially overexpressed in medullary carcinomas (MC, 19/21, 90\%) compared with $65 \%$ $(13 / 20)$ in non medullary adenocarcinomas ( $\mathrm{p} 0.06)$. PD-L1 expression by tumor cells 
was only observed in MSI CRC (19/41, 46\% with PD-L1 expression in more than $5 \%$ of tumor cells - score 1$)$, and preferentially in MC ( $57 \%$ vs $5 \%$ in no medullary adenocarcinomas, with PD-L1 expression in more than $50 \%$ of tumor cells - score 3 , $\mathrm{p}$ 0.0005). Conversely, PD-L1 expression by immune cells was observed in MSI CRC (23/41, 56\% with PD-L1 expression by more than 5 sheets of 50 positive cells) but also in MSS CRC (18/39, 43\%) (p 0.5$)$. The density of PD-1+ cells was significantly correlated to the PD-L1 expression, as well as the density of Tbet+ TIL.

Conclusions: PD-L1 expression is 1) heterogeneous in CRC, among CRC but also within the same tumor, 2) preferentially observed in MSI CRC (78\%), especially in MC $(90 \%)$, where PD-L1 is expressed by tumor cells, 3) correlated with the density of PD-1+ or T-bet+ TIL, and 4) observed in a significant proportion of MSS CRC (46\%) by immune cells only. From a clinical point of view, PD-L1 expression has to be determined at best in full tissue section and besides its preferential expression in MSI CRC, its significant frequency and expression profile (only by immune cells) in MSS CRC should be taken into account in the future clinical trials testing the efficacy of anti-PD-1/PD-L1 antibodies.

\section{$647 \quad$ Frequency of Deficient Mismatch Repair (MMR) Proteins and HNPCC in Patients with Colorectal Carcinoma (CRC) Younger and Older Than or Equal to 70 Years}

Azore-Dee Bradshaw, Margaret Cho, Gabriel Acosta Gonzalez, Richard A Hickman, Ruliang $X u$. New York University Langone Medical Center, New York, NY.

Background: The 2015 NCCN guidelines recommend screening either all CRC patients or those younger than 70 years old and those 70 years old and older who meet the Bethesda guidelines for HNPCC at the time of diagnosis. It is debatable whether patients 70 years and older with colorectal cancer are less likely to have HNPCC. We analyzed the frequency of MSI-high status and HNPCC among these two groups of patients to address this issue.

Design: We retrospectively searched our pathology database for patients who underwen resection, polypectomy and biopsy for CRC from 2013 to 2016. Patient age, gender, tumor site, immunohistochemistry (IHC) result for MMR proteins, and HNPCC status were recorded. IHC for MMR proteins was performed using Ventana kit (MLH1, MSH2, MSH6 and PMS2) and an automated system. Patients who only had MLH1 and PMS2 deficiency with positive BRAF mutation and hypermethylation were defined as low probability of HNPCC, whereas those who had either loss of PMS2 only, MSH2 and/or MSH6 were classified as high probability of HNPCC. Fisher's exact test was performed when appropriate.

Results: Among 316 patients identified, 191 patients were $<70$ years old, and 125 patients were $>/=70$ years old. Both groups had almost equal sex distribution (M:F ratio: $1.17: 1$ and $0.87: 1)$. In the younger age group, the tumor was predominantly left-sided $(\mathrm{n}=137,71 \%)$, whereas the older age group showed an equal distribution $(\mathrm{n}=62 \mathrm{vs}$. $\mathrm{n}=63$ ). The percentage of loss of MMR proteins detected by IHC in the younger group (19\% or $36 / 191)$ was not significantly different from that in the older age group $(23 \%$ or 29/125) ( $\mathrm{P}>0.05)$. There was also no significant difference between the percentage of patients with high probability of HNPCC in younger and older age groups ( $8 \%$ or $16 / 191$ vs. $2 \%$ or $3 / 125$ ). However, among the patients with loss of MMR proteins in the tumor, the younger age group had a significantly higher percentage of high probability of HNPCC $(44 \%$ or $16 / 36$ vs. $10 \%$ or $3 / 29)(\mathrm{P}<0.05)$.

Conclusions: In our institution, screening by IHC for MMR proteins identified similar percentages of HNPCC or highly probable HNPCC in younger and older than 70 year old patient groups, supporting universal screening for HNPCC regardless of age. There is a higher percentage of HNPCC or highly probable HNPCC among patients in the younger group who have loss of MMR proteins in the tumor, suggesting that the loss of MMR proteins in tumors in older patients is more likely an epigenetic event.

\section{Hirschsprung Disease and Its Mimickers: A Detailed} Histomorphological Study of 12 Cases

Maria Bukelo, Amanda C Pinto, Suravi Mohanty, P Divya, N B Nandeesh, K M Babu, Usha Kini. St. John's Medical College, Bengaluru, Karnataka, India.

Background: Hirschsprung disease(HD) most commonly presents in neonates with abdomen distension and constipation. Their rectal biopsy shows aganglionosis with the presence of hypertrophic nerve bundles (HNBs). However, there are many adults who clinically resemble $\mathrm{HD}$ despite the presence of ganglion cells(GCs) in their rectal biopsies. Various terms have been used to describe these conditions such as 'variants of HD', 'pseudo-HD' or 'chronic idiopathic pseudo-obstruction'. This study is aimed at a histomorphological evaluation of biopsies from adults who clinically presented as HD. Design: A retrospective 5 year histomorphological study of adult patients who presented with clinical features of Hirschsprung disease and no intestinal luminal pathology was done from our biopsy records.

Results: 12 adults aged between 21 and 55 years (mean age: 35 years) were selected. All 12 presented with abdominal distension and chronic constipation and 9 had a colostomy at some point in life. Frozen rectal mucosal biopsy in 10 patients showed one case of HD (21 year/male) with aganglionosis \& increased Acetylcholine Esterase (AChE) activity; 5 cases with GCs and no HNBs, reported as 1 . Normal innervation, 2. Hypoganglionosis, 3. Ganglioneuromatosis (Met918Thr mutation in exon 16 of the RET, confirmatory of MEN2B syndrome), 4. Lymphocytic ganglionitis and 5. refractory constipation with associated megarectum. One of 2 cases with GCs and prominent nerve bundles had a megacolon secondary to Pheochromocytoma while the other had minimal chronic inflammation probably due to an inflammatory pathology. Two cases with neither GCs nor HNBs with normal AChE activity were reported as normal innervation. Two cases did not have a rectal biopsy; the appendix in one showed GCs and neuromatosis confirmed by IHC, while in the other, a full thickness colonic biopsy showed the inner layer of muscularis propria with vacuolar change and atrophy with abnormal synaptophysin expression, altered distribution of ICC (CD 117) and depleted SMA expression. These features were compatible with the clinical diagnosis of myopathic type of intestinal pseudo-obstruction.

Conclusions: Adults presenting with symptoms of abdomen distension and chronic constipation should be evaluated for HD and its variants. Syndromic (MEN2B) and autoimmune eitiologies should be kept in mind. Myopathic causes should be evaluated with a full thickness colonic biopsy. Unlike HD, surgical excision of the abnormal bowel is not advocated as its proximal extent of the pathology is not assessable and hence the management is a challenge.

\section{Unique Prognostic Significance of CD206 and CD163} Macrophage Subtype Markers in Esophageal Adenocarcinoma

Wenqing Cao, Margaret A Black, Jiangzhou Yu. New York University Langone Medical Center, New York, NY; University of Illinois at Chicago, Chicago, IL.

Background: Detection macrophage subtype in tumor microenvironment could be prognostically useful. M2 macrophage markers may be tissue specific. CD163 and CD206 are often used in a variety of tumors to correlate M2-like macrophage in tissue with patient prognosis. Thus we compared the prognostic significance of CD163 or CD206 positive $\mathrm{M} 2$ macrophage in esophageal adenocarcinoma (EAC).

Design: EAC cell line SKTG was used to polarize THP1 monocyte into M2 macrophage. RT-PCR was employed to identify its cytokine expression profile. 53 EAC resection specimens were immunostained with CD68, CD163 and CD206. Average numbers of M2 (CD68+/CD163+) or (CD68+/CD206) macrophages were counted at five hot spots in tumor islet and tumor stoma, and correlated with clinicopathological factors. The association between M2 macrophage and overall survival was analyzed using KaplanMeier method. Other data analysis was done with Student $t$-test.

Results: M2 like macrophage induced by EAC cancer cell expressed high levels of CD163 and CD206. In EAC tissues, CD206 or CD163 positive cells were seen in tumor islet and stroma. Compared to EACs without lymph node metastasis, CD206 or CD163 positive M2-like macrophage counts in tumor islet and tumor stroma was not obviously increased in EACs with positive lymph nodes, and did not show a correlation with lymph node metastasis. Interestingly, CD163 positive M2-like macrophage possessed a strong correlation with patient survival independent of location $(\mathrm{p}<0.05)$. Counts of CD206- or CD163-like macrophage in EAC was not associated with patient age, gender, tumor size, and tumor differentiation.

Conclusions: CD163 is a suitable M2-like macrophage maker for macrophage subtype contributing to EAC progression and prognosis. Data also suggest choosing appropriate M2 macrophage marker is important in related cancer studies.

\section{Epidemiologic Evaluation of Biopsies Performed for Potential} Gastrointestinal GVHD

David Carr, Grace Y Lin, Mojgan Hosseini. UC San Diego, San Diego, CA.

Background: Gastrointestinal graft-vs-host disease (GVHD) is an important complication of allogeneic bone marrow transplantation and is common in both its acute and chronic forms. Severe gastrointestinal GVHD is an important diagnostic entity associated with high mortality. With the incidence of bone marrow transplantation increasing, and continued improvement in GVHD prophylactic regimens, a thorough understanding of the presentation and epidemiology of GVHD is crucial.

Design: The pathology archives were searched for gastrointestinal biopsies performed for evaluation of GVHD from 2011-2015. Cases were reviewed and classified based on NIH recommended diagnostic categories for GVHD (Negative for GVHD, Possible GVHD, and Likely GVHD). Clinical charts were reviewed for relevant data such as transplant indication, transplant type, time since transplant, endoscopic findings, clinical symptoms, prophylactic regimen, GVHD in other organs, and infectious status. Results: A total of 124 cases were identified. $54 \%$ of cases (67/124) were negative for GVHD. $9 \%$ of cases (11/124) were considered possible GVHD. 37\% of cases were considered likely GVHD (46/124) of which $28 \%$ were grade $1,24 \%$ grade $2,22 \%$ grade 3 , and $26 \%$ grade 4 . Of the negative cases, $12 \%(8 / 67)$ were due to infectious causes (CMV, EBV, adenovirus and $C$. difficile), one case was attributed to mycophenolate toxicity, and the majority $(87 \% ; 58 / 67)$ had no specific diagnostic alteration. 27 of 46 patients $(59 \%$; $95 \%$ CI $[52,66])$ with a diagnosis of likely GVHD were deceased at the time of chart review compared with 16 of 78 patients (21\%; 95\% CI [16-25]) categorized as either likely GVHD or negative for GVHD $(p<.001)$.

Conclusions: In this study, biopsies clinically suspicious for gastrointestinal GVHD were reviewed. We found a significant difference in mortality between patients with a positive GVHD biopsy of any grade compared to those with possible GVHD or negative biopsies. Overall, there was a relatively high positive biopsy rate for GVHD distributed over the spectrum of severity. Of the negative cases, a minority were attributed to infectious etiologies $(12 \%)$, however, the majority did not have any specific diagnostic alterations. In conclusion, we find a significant association between a diagnosis of GVHD of any grade and increased mortality. The positive biopsy rate is relatively high when there is clinical suspicion of gastrointestinal GVHD. Although infectious causes are an important consideration, the majority of negative biopsies will fail to yield a definitive diagnosis of other possible causative entities.

\section{Examination of Lymph Node Status in Gastroenteropancreatic} Neuroendocrine Tumors

Luis Carrillo-Polanco, Peter N Bonneau, Christopher Hartley, Catherine Hagen. Medical College of Wisconsin, Milwaukee, WI; University of Wisconsin Hospital and Clinics, Madison, WI.

Background: Presently, AJCC lymph node staging for gastroenteropancreatic (GEP) neuroendocrine tumors (NET) is classified as N0 (no lymph node metastases) or N1 (any lymph node metastases). In addition, previous studies have demonstrated examination of 
fewer than 12 lymph nodes in colonic adenocarcinoma cases is associated with a worse prognosis; this concept has not been explored in GEP NETs. The goal of this study was to determine if the number of examined lymph nodes or the number of positive lymph nodes is associated with prognosis in GEP NET.

Design: Our pathology database was searched from 2008-2013 for cases of GEP NET with a corresponding lymph node dissection. Tumor characteristics including number of lymph node metastases and number of lymph nodes examined were collected from corresponding pathology reports. Patient outcome data was collected from chart review. Results: The study group consisted of 84 GEP NET from an equal number of patients (38 small bowel/ampullary NET, 33 pancreatic NET (PanNET), 9 colorectal NET, 2 gastric NET, 2 appendiceal NET). The average number of lymph nodes examined per case was 17.8 (range 1-66), the average number of positive lymph nodes per case was 2.4 (range 0-18), and the average lymph node ratio per case was 0.19. PanNET had significantly more lymph nodes examined per case compared to other sites (mean 22.6 vs. 14.6, $\mathrm{p}=0.002$ ) but the lymph node ratio for PanNET was not significantly different $(0.15$ vs. $0.22, p=0.22)$. When all cases were included, disease free survival was not significantly different for cases with $<12$ lymph nodes examined compared to those with $\geq 12$ lymph nodes examined $(\mathrm{p}=0.90)$. Using the current AJCC staging criteria, there was some separation of patients with no lymph node metastases versus those with lymph node metastases on survival curves, although not significant $(\mathrm{p}=0.37)$. When positive lymph nodes were divided into 1 or $>1$, this separation became less pronounced $(\mathrm{p}=0.53$ ).
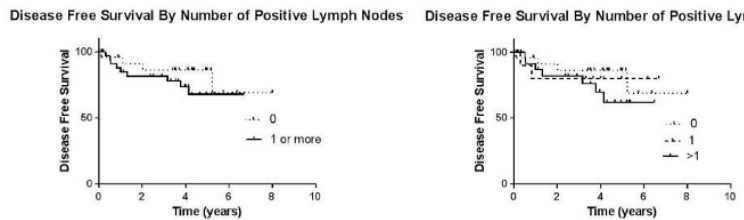

Conclusions: In these preliminary findings there is no evidence to suggest that the number of lymph nodes examined or number of positive lymph nodes are associated with disease free survival for GEP NET. We are building a larger cohort to strengthen our findings.

\section{Clinical Significance of Monoclonal T-Cell Populations in} Duodenal Lymphocytosis: Celiac and Non-Celiac Patients

Romulo Celli, Pei Hui, Sidney Bogardus, Marie Robert. Yale Medical School, New Haven, CT.

Background: Refractory Celiac disease (RCD) is defined as persistent duodenal villous blunting despite a gluten-free diet (GFD). Monoclonal T-cells in duodenal mucosa in RCD is a criterion for RCD type II, portending a risk for enteropathy associated T-cell lymphoma (EATL). T-cell receptor (TCR) gene rearrangement by PCR using paraffin-embedded tissue is a highly sensitive methodology, which may detect clinically insignificant clones. No studies have evaluated the specificity of monoclonal TCR gene status in non-RCD duodenal lymphocytosis. We assessed T-cell clonality status and clinical outcome in patients with RCD, celiac disease (CD) and Helicobacter pylori (HP) associated duodenal lymphocytosis.

Design: Duodenal biopsies from $12 \mathrm{RCD}$ patients, $11 \mathrm{CD}$ patients and 5 patients with HP associated duodenal lymphocytosis were identified in our files. TCR gene rearrangement analysis was performed by PCR. Clinical follow up was obtained.

Results: Clonal TCR gene rearrangement was detected in 6/12 RCD (50\%), 2/11 untreated $\mathrm{CD}(18 \%)$, and $2 / 5 \mathrm{HP}$ patients $(40 \%) .2 \mathrm{RCD}$ patients had identical clones in sequential biopsies. In sequential biopsies from $5 \mathrm{RCD}$ patients, multiple different clones were found. In RCD group: $2 / 12$ patients died of disease, 3 require imunosuppression, 2 are entering clinical trials, 5 take supplements. None developed EATL; 1 developed nodal B-cell lymphoma; 2 developed small bowel carcinoma (mean follow up 6.5 years, range $0-20$ ). Of 2 untreated CD patients with positive TCR, 1 improved on GFD; one had persistent disease, with uncertain GFD status. The HP infected patients had no malabsorption.

Table 1.Clonal TCR and Follow Up

\begin{tabular}{|l|l|l|l|}
\hline & RCD $(\mathrm{n}=12)$ & $\begin{array}{l}\text { New CD } \\
(\mathrm{n}=11)\end{array}$ & $\begin{array}{l}\text { HP } \\
(\mathrm{n}=5)\end{array}$ \\
\hline Mean Age (yrs) & 62 & 42 & 47 \\
\hline Gender (female, \%) & $9(75 \%)$ & $8(73 \%)$ & $\begin{array}{l}4 \\
(80 \%)\end{array}$ \\
\hline TCR, clone (n, \%) & $6 / 12(50 \%)$ & $2(18 \%)$ & $\begin{array}{l}2 \\
(40 \%)\end{array}$ \\
\hline $\begin{array}{l}\text { Sequential TCR (n=7) } \\
\text { Multiple Clones }\end{array}$ & $5 / 7$ & $2(18 \%)$ & $\begin{array}{l}2 \\
(40 \%)\end{array}$ \\
\hline Persistent identical clone & $2 / 7$ & 0 & 0 \\
\hline $\begin{array}{l}\text { Development of Malignancy } \\
(\mathrm{n}, \%)\end{array}$ & $\begin{array}{l}2 \text { Small bowel carcinoma } \\
(17 \%) \\
1 \text { nodal B cell lymphoma }(8 \%)\end{array}$ & 0 & 0 \\
\hline
\end{tabular}

Conclusions: Monoclonal T-cell populations are present in patients with RCD, newly diagnosed CD, and HP associated duodenal lymphocytosis. This finding highlights the clinical non-specificity of results of PCR TCR gene rearrangement using paraffin embedded tissue. Test results should be correlated with morphology, sequential biopsies, and clinical status. The finding of monoclonal T-cell populations in conditions of duodenal lymphocytosis is of limited clinical significance as a stand-alone test.
653 Dysplasia in Sessile Serrated Polyps Is Frequently Encountered in Patients Meeting Serrated Polyposis WHO Diagnostic Criteria

Romulo Celli, Joanna Gibson. Yale Medical School, New Haven, CT.

Background: Serrated polyposis syndrome (SPS) is defined by multiple colonic serrated polyps (SP) and increased risk of colorectal cancer (CRC). The 2010 WHO criteria for SPS are: 1) $\geq 5$ SP proximal to sigmoid with $\geq 2$ being $\geq 1 \mathrm{~cm} ; 2$ ) any SP proximal to sigmoid in a patient with a 1st-degree relative with SPS or 3) $\geq 20$ SP. SPS incidence is unknown and a confirmatory test is unavailable. We tested the utility of the WHO criteria in identifying patients with SPS in a large cohort.

Design: 2345 consecutive patients with at least 1 SP (hyperplastic polyp, HP; sessile serrated polyp, SSP; and traditional serrated adenoma, TSA) were identified via retrospective review of the pathology database over the year 2014. Each patient was screened for SPS and clinicopathologic examination was performed of patients meeting WHO criteria.

Results: We identified 32 patients ( 18 female, mean age 67 years) who met SPS WHO criteria. 3 patients met criteria at the 1 st colonoscopy; remaining patients met criteria after tabulating data from a mean of 3.5 procedures. 20 patients met criterion 1 (Group A), 7 met criterion 3 (Group B), and 5 met both 1 and 3. The mean number, size, anatomic distribution and histologic type of SP differed significantly between Group A and $B(10.5$ vs $30.4, p=0.00 ; 0.77 \mathrm{~cm}$ vs $0.41 \mathrm{~cm}, \mathrm{p}=0.00,29 \%$ vs $6 \%$ right sided, $\mathrm{p}=0.00$; and $39 \%$ vs $16 \%$ SSP, $\mathrm{p}=0.00$, respectively). $13 \%$ of SPS patients had SSP with advanced neoplasia (SSP-AN), including 1 with high grade dysplasia and 3 with CRC, compared to $0.9 \%$ of the non-SPS patients in this cohort. The mean number of polyps in patients with SSP-AN was 23 , and no patient with SSP-AN had less than 10 polyps. Conclusions: We identified 32 patients meeting SPS criteria among 2345 patients with at least one SP (1.4\% incidence). SP histology, size and anatomic distribution varied between patients meeting criterion 1 or 3 , confirming others' findings of 2 SPS phenotypes and validating WHO criteria for identifying both. We did not identify patients meeting criterion 2, a limitation of our study design. $13 \%$ of SPS patients had SSP-AN, supporting the increased risk of neoplastic progression in patients with SPS compared to patients without SPS. Most patients met SPS criteria after 2 or more colonoscopies, and SSP-AN occurred in SPS patients with greater number of SP. Our studies also suggest that SSP-AN may be an additional criterion for diagnosis of SPS. We recommend pathologists routinely reviewing prior pathology and medical records of patients with a diagnosis of any SP, including dysplastic SSP, to screen for SPS.

654 Interobserver Variability in the Diagnosis of Inflammatory Bowel Disease-Associated Dysplasia by International Telepathology

Michael Chang, Yanfei Huang, Dipti M Karamchandani, Xian-rui Wu, Daniela Allende, John R Goldblum, Shu-Yuan Xiao, Hongfa Zhu, Michael Feely, Amy Collinsworth, Ashwini K Esnakula, Hao Xie, Xiuli Liu. U of Florida, Gainesville, FL; Penn State U, Hershey, PA; Sun Yat-sen U, Guangzhou, China; Cleveland Clinic, Cleveland, OH; U of Chicago, Chicago, IL; Mount Sinai Hosp, New York, NY; Yale U, New Haven, CT. Background: Dysplasia arising in association with inflammatory bowel disease (IBD) is the most significant marker of increased colorectal cancer risk in IBD patients. Telepathology (TP), the practice of remote diagnostic consultation, has seen increased use nationally and internationally. The aim of this study was to evaluate the utility and interobserver variability of diagnosing dysplasia in IBD with TP.

Design: Eight gastrointestinal (GI) pathologists were involved in this study. A total of 50 colonic biopsies from patients with IBD were included. One representative microscopic slide in each case was digitized using an Aperio system. Photographs of the full slide images were captured at low, medium, and high magnifications at a resolution of $1712 \times 1072$ pixels and saved as tagged image file format (TIFF) files on read-only DVD. Each pathologist selected the most appropriate diagnostic category for each case; a consensus diagnosis was defined as diagnostic agreement by at least 4 pathologists and included negative $(n=22)$, low-grade dysplasia $(n=22)$, high-grade dysplasia $(\mathrm{n}=3)$, cancer $(\mathrm{n}=2)$, and indefinite $(\mathrm{n}=1)$. A kappa coefficient analysis was performed to determine interobserver agreement and the agreement of each pathologist with the consensus diagnosis.

Results: There was moderate interobserver agreement among 8 pathologists (kappa value $0.58[0.50,0.67])$. Agreement of each pathologist with the reference consensus diagnosis revealed kappa values ranging from 0.53 to 0.84 .

\begin{tabular}{|l|l|l|l|}
\hline Reviewer number & Practice experience (years) & Kappa & Agreement \\
\hline 1 & $<5$ & 0.53 & Moderate \\
\hline 2 & $<5$ & 0.70 & Substantial \\
\hline 3 & $<5$ & 0.75 & Substantial \\
\hline 4 & $<5$ & 0.64 & Substantial \\
\hline 5 & $>8$ & 0.84 & Almost perfect \\
\hline 6 & $>8$ & 0.74 & Substantial \\
\hline 7 & $>8$ & 0.84 & Almost perfect \\
\hline 8 & $>8$ & 0.81 & Almost perfect \\
\hline
\end{tabular}

GI pathologists with more than 8 years of practice experience achieved greater agreement with each other than those with less than 5 years of practice experience (kappa 0.69 vs. 0.50 , respectively, $\mathrm{p}<0.001$ ).

Conclusions: This pilot study reveals moderate interobserver agreement on the interpretation of colonic dysplasia in IBD using digitized images. Greater practice experience seems to improve interobserver agreement. This study supports the efficacy of international consultation by digital pathology. 
$655 \quad H y d r o p h i l i c$ Polymer Associated Ischemic Enterocolitis Jesus A Chavez, Wei Chen, Wendy L Frankel, Christina A Arnold. The Ohio State University, Columbus, $\mathrm{OH}$.

Background: Hydrophilic polymer coating of medical devices serves to lubricate the device and prevent device-related complications. The coating can be disrupted and result in downstream pathology via presumed thromboembolism. This process has been reported in the brain, heart, lung, and skin, and has been replicated through anima studies and in vitro histologic processing of the polymer coating. We report the first description of hydrophilic polymer associated ischemic enterocolitis.

Design: Prospective identification of the polymers from gastrointestinal resection specimens from 04/29/2014-08/08/2016 resulted in collection of seven specimens (small bowel $=2$, colon $=4$, aortic thrombus $=1$ ) from three patients.

Results: We report a 4\% incidence of hydrophilic polymer associated ischemic enterocolitis among all patients with an ischemic bowel resection during this study. All patients developed bowel ischemia within one day of aortic repair. All resection specimens showed ischemia, and the polymers were mainly located in the submucosal vessels in areas of ischemia. They appeared as intravascular, serpiginous structures with basophilia. In the patient who developed acute paralysis after the aortic repair, identical polymers were identified in the aortic thrombus and the ischemic bowel segment. We demonstrate that the polymers display an altered morphology over time and with various graft types, and that the degrading polymers are associated with foreign body giant cell reaction. Special stains can be helpful with the polymers turquoise on a colloidal iron, pink on a von Kossa and mucicarmine, and pale blue on a trichrome. Clinical follow-up was available up to 115 weeks: one patient died, and the other two are alive and well. Conclusions: In summary, we report a new diagnostic entity to be considered in the differential of iatrogenic ischemic injuries. Awareness of this entity is important to elucidate the cause of ischemia and to prevent misdiagnosis of the polymers and their associated giant cell reaction as a parasitic infection, granulomatous vasculitis, sarcoidosis, and idiopathic inflammatory bowel disease.

656 Assessment of PD-L1 Expression in Tumor Microenvironment of Mismatch Repair Deficient Colon Cancer: Comparing Two Antibody Clones, SP142 vs. SP263

Zongming E Chen, Angela Bitting, Fan Lin. Geisinger Medical Center, Danville, PA. Background: Mismatchrepair deficient (MMRD) colon cancer harbors increased number of somatic mutations and is potentially more immunogenic. To evade hos immune destruction, the tumor cells utilize check point inhibition mechanisms. Consequently, PD-L1 expression in the tumor microenvironment exhibits prognostic significance in this subtype; and majority patients benefit from anti-PD1 immunotherapy. However, the evaluation of PD-L1 expression may differ depending on different antibody clones. Here, we compared the results using two PD-L1 clones SP142 and SP263.

Design: Immunohistochemistry (IHC) for PD-L1 was performed on 40 MMRD colon adenocarcinomas using tissue microarray sections. The tumors were selected for their lack of MLH1 and PMS2 expression by IHC and positive for BRAF V600E mutation. Two PD-L1 antibodies (clones SP142 and SP263, Ventana, AZ, USA) were optimized according to the vender's protocols and used in assays. A four-tiered grading system was used for evaluating PD-L1 expression on immune and tumor cells, respectively (Table 1). Statistical analysis was performed using two-tailed Fisher exact test.

Results: A significant PD-L1 expression was detected in the tumor microenvironment by both antibodies (Table 1). However, the detection sensitivity for tumor cells was significantly lower using SP142 compared to SP263, particularly in low level of expressers. Although no statistically different in detecting PD-L1 expression in immune cells between the two antibodies, in most cases, the evaluation and scoring was easier using SP142 in the absence of interference from coexisting tumor cell staining pattern

\begin{tabular}{|c|c|c|c|c|c|c|}
\hline \multirow{2}{*}{$\begin{array}{l}\text { Antibody } \\
\text { clones }\end{array}$} & \multicolumn{3}{|c|}{$\begin{array}{l}\text { Stromal and intratumoral immune cells } \\
40 \text { tumors in total }\end{array}$} & \multicolumn{3}{|c|}{$\begin{array}{c}\text { Tumor cells } \\
40 \text { tumors in total }\end{array}$} \\
\hline & $<1 \%$ & $1-5 \%$ & $>5 \%$ & $<1 \%$ & $1-5 \%$ & $>5 \%$ \\
\hline SP142 & 12 & 3 & 25 & 34 & 3 & 3 \\
\hline SP263 & 10 & 10 & 20 & 17 & 12 & 11 \\
\hline${ }^{*}$ p-value & N/A & 0.80 & .37 & N/A & 0.01 & $<0.01$ \\
\hline
\end{tabular}

Conclusions: PD-L1 expression is upregulated in tumor microenvironment of MMRD colon cancer. Depending on the choice of specific antibody clones, significant difference may exist in the evaluation of PD-L1 expression in tumor and immune cells. This variation should be considered when applying PD-L1 expression as a prognostic or predictive biomarker for clinical use.

657 Mucinous Variant of Intrahepatic Cholangiocarcinoma: A Clinicopathological and Immunochemical Study

Zhikai Chi, Amarpreet Bhalla, Hanlin L Wang, Deepa T Patil, Jingmei Lin. Indiana University School of Medicine, Indianapolis, IN; University of California David Geffen School of Medicine, Los Angeles, CA; Robert J. Tomsich Pathology and Laboratory Medicine Institute, Cleveland, $\mathrm{OH}$.

Background: Mucinous variant of intrahepatic cholangiocarcinoma (iCC) is not common. The clinicopathological features and prognosis are far less clear. Design: Six patients who had iCCs with more than $50 \%$ of mucinous differentiation were included in the study that was compared to 81 cases of conventional iCCs. Results: The mean ages in mucinous and conventional iCC groups were 59 (range, $40-82$ ) and 61 (range, 52-82), respectively $(p>0.05)$. The female to male ratio was 0.5 in mucinous group compared to 1.4 in conventional iCC $(p>0.05)$. The size of tumor was $6.2 \mathrm{~cm}$ in mucinous variant which was similar $(6.0 \mathrm{~cm})$ in the conventional group $(p>0.05)$. Majority patients with mucinous iCC $(83 \%)$ presented as at least stage T3, compared to $28 \%$ in the conventional group $(p<0.05)$. Follow-up study showed 3 patients with mucinous variant $(50 \%)$ died of disease $(0-1$ year) with 1-year surviva rate of $17 \%$ compared with $26 \%$ of the conventional group. The average survival time of patients with mucinous iCC was significantly reduced compared to the conventional group $(0.17$ vs 2 years; $p<0.05)$. Compared with 6 conventional iCC cases with matched age, sex and tumor stage , immunohistochemical analysis revealed positive immunoreactivity in MUC1 ( $83 \%$ vs $33 \%)$, MUC2 (29\% vs 0$)$; MUC5AC ( $86 \%$ vs $67 \%)$, MUC6 ( $43 \%$ vs 0$)$, CK7 ( $71 \%$ vs $67 \%)$, CK20 ( 0 vs $33 \%)$, and CDX2 $(14 \%$ vs 0 ) in mucinous and conventional iCCs, respectively. Immunohistochemistry for PMS2 and MSH6 showed intact nuclear staining in all iCC cases, including mucinous variants. P53 showed 66\% immunoreactivity in both mucinous and conventional groups. Conclusions: Mucinous variant constitutes $7.4 \%$ of primary iCCs. Immunophenotypically, mucinous variant is reactive for MUC1, MUC2 and MUC6 that is devoid in the conventional type. Unlike gastrointestinal mucinous adenocarcinoma, mucinous variant of iCCs are often $\mathrm{CK} 7+/ \mathrm{CK} 20$ - and microsatellite stable. Mucinous iCCs likely present at advanced stage upon diagnosis with worse prognosis compared to the conventional counterparts.

658 Target?

Zhikai Chi, Romil Saxena. Indiana University School of Medicine, Indianapolis, IN. Background: Corticosteroids form the mainstay of therapy for microscopic colitis in patients with severe symptoms. However, the relapse rate is considerably high when therapy is discontinued. Long term steroid therapy, on the other hand predisposes to steroid dependence and significant side effects. Identification of specific targets and alternate directed therapies is therefore highly desirable.

Design: Colonic biopsies of collagenous colitis $(\mathrm{n}=29)$ or lymphocytic colitis $(\mathrm{n}=35)$ were identified. Twenty colonic biopsies with no pathologic change served as controls. Immunohistochemistry for tryptase was performed in all cases. Number of mast cells in lamina propria, muscularis mucosae and submucosa in fields with the highest and lowest density were counted. Presence of extracellular tryptase was recorded. The number of fragments that showed inflammation were counted in each case to assess the extent of inflammation.

Results:

\begin{tabular}{|c|c|c|c|c|}
\hline \multicolumn{2}{|l|}{ 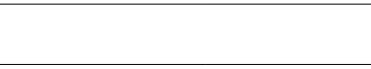 } & $\begin{array}{l}\text { Lymphocytic } \\
\text { colitis }(\mathrm{n}=35)\end{array}$ & $\begin{array}{l}\text { Collagenous } \\
\text { Colitis }(\mathrm{n}=29)\end{array}$ & $\begin{array}{l}\text { Controls } \\
(\mathrm{n}=20)\end{array}$ \\
\hline \multicolumn{2}{|l|}{ Age (mean, range) } & $69(45-93)$ & $68(49-89)$ & $61(52-93)$ \\
\hline \multicolumn{2}{|l|}{ Females $(\%, \mathrm{n})$} & $68 \%(24)$ & $90 \%(26)$ & $70 \%(14)$ \\
\hline \multirow{3}{*}{$\begin{array}{l}\text { Highest count per } \\
\text { high power field } \\
\text { (mean, range) }\end{array}$} & Lamina Propria & $30(13-42)^{* *}$ & $39(19-63)^{* * *}$ & $23(10-37)$ \\
\hline & $\begin{array}{l}\text { Muscularis } \\
\text { Mucosae }\end{array}$ & $1(0-3)$ & $1(0-3)$ & $1(0-1)$ \\
\hline & Submucosa & $12(3-26)$ & $15(4-37)$ & $10(3-22)$ \\
\hline \multirow{3}{*}{$\begin{array}{l}\text { Lowest Count } \\
\text { per high power } \\
\text { field(mean, range) }\end{array}$} & Lamina Propria & $17(6-27)^{* *}$ & $20(8-45)^{* *}$ & $12(3-25)$ \\
\hline & $\begin{array}{l}\text { Muscularis } \\
\text { Mucosae }\end{array}$ & $0(0-1)$ & $0(0-1)$ & $0(0-1)$ \\
\hline & Submucosa & $7(0-21)$ & $8(1-25)$ & $6(3-11)$ \\
\hline \multicolumn{2}{|c|}{ Extracellular tryptase present $(\%, n)$} & $60 \%(21)^{* * * *}$ & $41 \%(12)^{*}$ & $10 \%(2)$ \\
\hline \multicolumn{2}{|c|}{$\%$ of inflammed fragments (range) } & $89 \%(44-100)$ & $93 \%(63-100)$ & \\
\hline \multirow{2}{*}{$\begin{array}{l}\text { Mean highest count } \\
\text { per high power field } \\
\text { in lamina propria } \\
\text { (range) }\end{array}$} & $\begin{array}{l}<80 \% \text { inflammed } \\
\text { fragments }\end{array}$ & $22(13-30 ; \mathrm{n}=8)$ & $\begin{array}{l}45(31- \\
59 ; \mathrm{n}=4)^{* * *} \\
\end{array}$ & \\
\hline & $\begin{array}{l}>80 \% \text { inflammed } \\
\text { fragments }\end{array}$ & $\begin{array}{l}32(17-42 ; \\
n=27)^{* *}\end{array}$ & $\begin{array}{l}38(19- \\
63 ; \mathrm{n}=25)^{* *}\end{array}$ & \\
\hline
\end{tabular}

${ }^{*} \mathrm{p}<0.05, * * \mathrm{p}<0.01, * * * \mathrm{p}<0.001$

Conclusions: The pathogenesis of microscopic colitis is largely unknown and likely to be multifactorial; our findings suggest that mast cells may play a role.

Therapies targeting mast cells, especially those that stabilize cell membranes and prevent degranualtion, may be effective in these patients, thus precluding the need for long term steroid therapy.

Evaluation of mast cell numbers, presence of extracellular tryptase, and extent of inflammation might identify patients most likely to respond to such therapies.

659 Programmed Cell Death-Ligand 1 Expression in Microsatellite Instability-High Gastric Carcinomas

Junhun Cho, Young Hwan Chang, Hyunsik Bae, Kyoung-Mee Kim. Soonchunhyang University Cheonan Hospital, Soonchunhyang University College of Medicine, Cheonan, Korea; Oregon Health \& Science University, Portland, OR; Samsung Medical Center, Sungkyunkwan University School of Medicine, Seoul, Korea.

Background: Programmed death-ligand 1 (PD-L1) is expressed in a subgroup of gastric cancer(GC) that may benefit from immunotherapy. Microsatellite instabilityhigh (MSI) is a potential predictive factor for the response of immune therapy targeting PD-1/PD-L1. However, the interaction between PD-L1 expression and MSI remains poorly understood.

Design: We investigated PD-L1 expression in 78 MSI GC tissues using immunohistochemistry.

Results: Based on PD-L1 expression status, we classified PD-L1 expression cases into tumor cell (PD-L1 ${ }^{\mathrm{TC}+/}$ ) and immune cell $\left(\mathrm{PD}-\mathrm{L} 1^{\mathrm{IC+}+}\right.$ ) expression by pathologic examinations and the results were compared to those of digital automatic quantifications using acquired images with an Aperio slide scanner. Each stain was artificially attributed a color code, and images were overlaid using ImageJ. Pixel colocalization 
was assessed by calculating Manders' overlap coefficient with threshold set by Costes method (tM) using Fiji (Coloc 2 plug-in). We perform cell segmentation using Otsu's method and define PD-L1 positive cell ratio as an absolute number of positive cells per square millimeter. With pathologic observation, we observed PD-L1 expression in $48(61.5 \%)$ cases consisting $7(9.0 \%)$ PD-L $1^{\mathrm{TC}+}$ and $47(60.3 \%)$ PD-L $1^{\mathrm{IC}+}$. PD-L $1^{\mathrm{IC}-}$ was frequently associated with higher lymph node metastasis $(\mathrm{p}=0.027)$ and advanced TNM stages $(\mathrm{p}=0.029)$. Moreover, $\mathrm{PD}-\mathrm{L} 1^{\mathrm{IC}+}$ is an independent favorable prognostic factor in overall survival (versus PD-L $1^{\text {IC-- }}$, hazard ratio, $3.451 ; 95 \%$ confidence interval, $1.172-12.745 ; \mathrm{p}=0.025)$. We found significant correlation between manual and automatic quantifications ( $\mathrm{r}=0.8$ and $\mathrm{P}<0.001$, Spearman test) of PD-L1 positive cells.

Conclusions: In MSI-high GC, PD-L1 expression in immune cells is independently associated with longer survival.

\section{IgG4-Related Disease of the Gastrointestinal Tract: A Tertiary} Care Hospital Experience

Woo Cheal Cho, Saverio Ligato, Richard Cartun, Anshu Trivedi. Hartford Hospital, Hartford, CT.

Background: IgG4-related disease (RD) is a newly recognized rare clinicopathologic entity characterized by dense fibrosis \& lymphoplasmacytic infiltration rich in IgG4positive plasma cells in association with an elevated serum IgG4 level. We report our 10-year experience with IgG4-RD as a tertiary care hospital, with a primary focus on the gastrointestinal tract, \& discuss the challenges associated with the diagnosis.

Design: We searched our laboratory database for patients with suspected IgG4-RD encountered over a 10-year period (2006-2016), using the following terms: "IgG4," "autoimmune pancreatitis," "autoimmune sclerosing cholangitis," "retroperitoneal fibrosis," and "inflammatory pseudotumor." All slides and electronic medical records were retrospectively reviewed.

Results: The material examined consisted of 11 surgical and 5 biopsy specimens, all of which were histologically confirmed to have IgG4-RD in at least 1 organ. $63 \%$ were male and the median age at diagnosis was 60.5 years (range, 15-79). The primary sites in order of frequency were as follows: pancreas (6), mesentery (5), bile duct (3), retroperitoneum (3), \& gallbladder (1). Serum IgG4 levels were obtained in $19 \%$ (3/16) of patients, all of whom were found to have elevated serum IgG4 levels. In 1 patient, a serum total IgG level was obtained instead, which was elevated at $1852 \mathrm{mg} /$ dL. $13 \%(2 / 16)$ of patients had a history of malignancy and $6 \%(1 / 16)$ of patients had another documented autoimmune disease. Of 6 patients with IgG4-related sclerosing pancreatitis, $33 \%(2 / 6)$ of them had a history of alcohol abuse. $13 \%(2 / 16)$ of patients were treated with steroid alone or combination of steroid \& immunosuppressive therapy. Of 2 patients who received treatment, 1 patient experienced a relapse.

Conclusions: In our study, only a small portion of patients were further tested by serology \& received treatment with steroid following tissue diagnosis. Although histology remains the gold standard for the diagnosis of IgG4-RD, the complete spectrum of histologic changes may not be seen, particularly in a biopsy specimen. Given the fact that IgG4-RD is steroid-responsive, increased awareness of this entity among clinicians is crucial not only for accurate \& timely diagnosis but also for proper management.

\begin{tabular}{|c|c|c|c|c|c|c|}
\hline $\begin{array}{l}\text { Patient } \\
\text { No. }\end{array}$ & $\begin{array}{c}\text { Age } \\
\text { (years) }\end{array}$ & Sex & Site(s) of involvement & $\begin{array}{c}\text { Type of } \\
\text { specimen }\end{array}$ & $\begin{array}{c}\text { Serum IgG4 } \\
\text { level } \\
\text { (mg/dL) }\end{array}$ & $\begin{array}{l}\text { Treatment } \\
\text { with } \\
\text { steroid }\end{array}$ \\
\hline 1 & 74 & M & Pancreas & $\mathrm{s}$ & - & $\mathrm{N}$ \\
\hline 2 & 73 & M & $\begin{array}{l}\text { Pancreas, bile duct, } \\
\text { gallbladder }\end{array}$ & $\mathrm{s}$ & - & $\mathrm{N}$ \\
\hline 3 & 47 & M & Pancreas & $B$ & - & $\mathrm{N}$ \\
\hline 4 & 72 & $\mathrm{~F}$ & Pancreas & $B$ & $\cdot$ & $\mathrm{N}$ \\
\hline 5 & 51 & M & Pancreas & $\mathrm{s}$ & $\cdot$ & $\mathrm{N}$ \\
\hline 6 & 79 & $\mathrm{~F}$ & Pancreas & $\mathrm{s}$ & - & $\mathrm{N}$ \\
\hline 7 & 64 & $\mathrm{~F}$ & Bile duct & S & $\begin{array}{c}104.9[4.0- \\
86.0]\end{array}$ & $\mathrm{N}$ \\
\hline 8 & 59 & M & Bile duct & $\mathrm{s}$ & $\begin{array}{c}539[4.0- \\
86.0]\end{array}$ & $\mathrm{N}$ \\
\hline 9 & 15 & M & $\begin{array}{c}\text { Mesentery } \\
\text { (periduodenal) }\end{array}$ & B & $\begin{array}{c}271.9[11.0- \\
157.0]\end{array}$ & $\mathrm{Y}$ \\
\hline 10 & 25 & M & $\begin{array}{c}\text { Mesentery } \\
\text { (periappendiceal) }\end{array}$ & $\mathrm{s}$ & - & $\mathrm{Y}(\&$ 6-MP) \\
\hline 11 & 61 & M & Mesentery (pericolonic) & $\mathrm{s}$ & - & $\mathrm{N}$ \\
\hline 12 & 68 & $\mathrm{~F}$ & Mesentery (periileal) & $\mathrm{s}$ & - & $\mathrm{N}$ \\
\hline 13 & 61 & M & Mesentery (pericolonic) & $\mathrm{s}$ & $\cdot$ & $\mathrm{N}$ \\
\hline 14 & 46 & M & Retroperitoneum & $B$ & - & $\mathrm{N}$ \\
\hline 15 & 41 & $\mathrm{~F}$ & Retroperitoneum & $\mathrm{s}$ & - & $\mathrm{N}$ \\
\hline 16 & 60 & $\mathrm{~F}$ & \begin{tabular}{|l} 
Retroperitoneum \\
\end{tabular} & $\mathrm{B}$ & 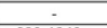 & $\mathrm{N}$ \\
\hline \multicolumn{7}{|c|}{$\begin{array}{l}\text { Patient } 1 \text { had a markedly elevated serum lgG level of } 1852 \mathrm{mg} / \mathrm{dL} \text { (reference, } 639-1349 \mathrm{mg} / \mathrm{dL} \text { ), but } \\
\text { serum lgG4 level was not obtained. History of autoimmune disease was seen in Patient } 7 \text {. History of } \\
\text { malignancy was seen in Patients } 18 \text {. 7. History of alcohol abuse was seen in Patients } 28 \text { \&. Relapse } \\
\text { was seen in Patient } 10 \text { ( } 2 \text { months following treatment). } 6 \text {-MP, } 6 \text {-mercaptopurine; } S \text {, surgical; B, biopsy. }\end{array}$} \\
\hline
\end{tabular}

661 DNA Flow Cytometric Analysis of Barrett's Esophagus-Related Dysplasia Using Paraffin-Embedded Tissue: DNA Content Abnormality Can Serve as Both Diagnostic Marker of Dysplasia and Predictive Marker of Neoplastic Progression

Won-Tak Choi, Peter S Rabinovitch, Thomas Small, Danning Huang, Aras N Mattis, Sanjay Kakar. UCSF Medical Center, San Francisco, CA; University of Washington, Seattle, WA; SUNY Upstate Medical University, Syracuse, NY.

Background: The diagnosis of dysplasia in Barrett's esophagus (BE) can be challenging, and reliable ancillary techniques are not available. Flow cytometry for DNA content has been shown to be a good biomarker for predicting esophageal adenocarcinoma (EAC). These studies have used fresh tissue, which is difficult in routine practice and does not allow correlation of morphologic findings with flow cytometry results. This study examines the utility of DNA content analysis by flow cytometry in formalin-fixed paraffin-embedded (FFPE) tissue in BE with dysplasia.
Design: DNA flow cytometry was performed using FFPE tissue from 78 high grade dysplasia (HGD) (36 biopsies, 42 resections), 14 low grade dysplasia (LGD) (11 biopsies, 3 resections), and $9 \mathrm{BE}$ without dysplasia. Three to four 60 micron thick sections were cut from each block, and area of interest was dissected for analysis. Results: DNA content abnormality (aneuploidy and/or elevated 4N fraction) was identified in $74(95 \%)$ HGD, 5 (36\%) LGD, and no BE case without dysplasia. The DNA content abnormality was similar in biopsies (94\%) and resections (95\%) in HGD cases. Seventy-three percent of LGD cases with DNA content abnormality progressed to $\mathrm{HGD} / \mathrm{EAC}$ at 1 year, with $100 \%$ progression at 4 years $(\mathrm{p}=0.037)$. By contrast, the 1 -year detection rate of $\mathrm{HGD} / \mathrm{EAC}$ for LGD cases in the setting of normal DNA flow cytometric findings was $17 \%$, which remained unchanged at 12 years. The hazard ratio for subsequent detection of HGD/EAC for patients with DNA content abnormality detected at baseline LGD was 7.23 ( $p=0.039 ; 95 \%$ confidence interval, $1.065-141.52$ ). Conclusions: This study demonstrates the promise of flow cytometry using FFPE tissue in the diagnosis and risk stratification of dysplasia in BE. The majority of HGD cases (95\%) show DNA content abnormality. LGD with DNA content abnormality is at higher risk for progression to $\mathrm{HGD} / \mathrm{EAC}$.

\begin{tabular}{|c|c|c|c|}
\hline & $\begin{array}{c}\text { No dysplasia } \\
(\mathrm{n}=9)\end{array}$ & LGD (n=14) & $\begin{array}{c}\text { HGD } \\
(\mathrm{n}=78)\end{array}$ \\
\hline DNA content abnormality & $0(0 \%)$ & $5(36 \%)$ & $74(95 \%)$ \\
\hline $\begin{array}{c}\text { Progression to HGD/EAC } \\
\text { at 1 year }\end{array}$ & - & $\begin{array}{c}73 \%\left(\begin{array}{c}\text { (with abnormal DNA } \\
\text { content) }\end{array}\right. \\
17 \%\left(\begin{array}{c}\text { (without abnormal DNA } \\
\text { content) }\end{array}\right.\end{array}$ & - \\
\hline
\end{tabular}

\section{Gastric Pyloric Gland Adenoma: A Multicenter Clinicopathologic} Study of 65 Cases

Won-Tak Choi, Ian Brown, Tetsuo Ushiku, Masato Yozu, Namrata Setia, Amitabh Srivastava, Melanie Johncilla, Rish K Pai, Masashi Fukayama, Joseph Misdraji, Gregory Y Lauwers. UCSF Medical Center, San Francisco, CA; Envoi Pathology, Kelvin Grove Qld, Australia; University of Tokyo, Tokyo, Japan; Massachusetts General Hospital, Boston, MA; University of Chicago, Chicago, IL; Brigham and Women's Hospital, Boston, MA; Mayo Clinic, Phoenix, AZ.

Background: Gastric pyloric gland adenoma (PGA) is a rare neoplasm known to occur in the setting of autoimmune gastritis (AIG), familial adenomatous polyposis (FAP), or Lynch syndrome. Although PGA may have a high malignant potential, it remains underrecognized, and there is limited information regarding its clinicopathologic features and risk factors for high-grade dysplasia (HGD) or adenocarcinoma.

Design: 65 PGAs from 56 patients were reviewed, and immunohistochemistry for MUC6 (pyloric gland mucin marker) and MUC5AC (foveolar mucin marker) was performed.

Results: PGA occurred more commonly in females (52\%) with a mean age of 68 . It usually appeared as a polypoid lesion $(80 \%)$ in the gastric body $(48 \%)$ but also presented as a mass $(6 \%)$, dome-shaped lesion $(5 \%)$, or nodule $(3 \%)$ in other parts of stomach: fundus $(15 \%)$, cardia $(8 \%)$, antrum $(6 \%)$, or pylorus $(3 \%)$. Among 45 PGAs with available background mucosa, 12 cases $(27 \%)$ developed in a background of AIG; the remaining $73 \%$ was not associated with AIG, including 16 in normal mucosa $(36 \%)$. Five patients $(9 \%)$ had FAP. The average size of PGAs was $2.3 \mathrm{~cm}$. Thirty-six of 65 cases (55\%) showed low-grade dysplasia (LGD) with an average size of $1.7 \mathrm{~cm}$, while 24 cases of HGD (37\%) were larger (average $3.4 \mathrm{~cm}$ ) including 8 cases $(33 \%)$ with adenocarcinoma. Tubulovillous architecture was more common in HGD (52\%) than LGD cases $(33 \%)$. In contrast with other series that reported a predominant pyloric-type (MUC6+, $<30 \%$ MUC5AC + ) as the most common phenotype, typically arising in a background of AIG, our series showed that the majority of PGAs $(51 \%)$ coexpressed MUC5AC with MUC6 in an intermixed pattern, followed by $24 \%$ of a predominant pyloric-type and $22 \%$ of a pure pyloric-type. PGAs with HGD and/or adenocarcinoma were more frequently associated with AIG (37\%) compared to those with LGD (24\%). Among 45 patients with known clinical follow-up, Only 3 patients $(7 \%)$ had a recurrence within a year.

Conclusions: PGA frequently demonstrates a combination of pyloric and foveolar epithelium and coexpresses MUC5A with MUC6 (mixed type). Many cases are not associated with AIG or a genetic syndrome. Although the occurrence of HGD and/or adenocarcinoma is increased with the size of the lesion and association with AIG and tubulovillous architecture, recurrence is low.

663 Concordance Between Mismatch Repair Status from Primary Colorectal Carcinoma and Distant Metastasis

Lani K Clinton, Thomas Plesec. Cleveland Clinic, Cleveland, $\mathrm{OH}$.

Background: Approximately $50-60 \%$ of colorectal cancer (CRC) patients develop metastases; often these patients are not surgical candidates. In these stage IV CRC patients, DNA mismatch repair (MMR) status helps guide medical management. In a recent phase II clinical trial, patients with MMR deficient CRCs responded significantly better to the anti-programmed death 1 (PD-1) inhibitor, pembrolizumab, than patients with MMR competent tumors. However, there are very limited data on the concordance of MMR status between primary CRC and distant metastasis, which causes uncertainty regarding the best tissue to assess for MMR status.

Design: After IRB approval, we reviewed specimens from patients who underwent extended $R A S$ testing (between $3 / 14-8 / 16$ ), indicating stage IV disease. Initial MMR testing was done by polymerase chain reaction (PCR), immunohistochemistry (IHC), or both. IHC for PMS2 and MSH6 was performed on the metastases, given the wellestablished and robust correlation of PMS2 and MSH6 expression with MSI PCR status and expression of all 4 MMR proteins by IHC. 
Results: Among 86 patients with metastatic CRC, 54 (63\%) had sufficient material for MMR IHC. Thirty (94\%) insufficient cases were cytologic specimens that were previously used for other ancillary tests. Methodology for MMR testing in the primary tumor included 34 (63\%) PCR/IHC, 14 (26\%) IHC only, and 6 (11\%) PCR only. Among 54 patients with sufficient metastatic tissue for MMR testing, 49 (92\%) had tissue biopsies/resections, and $5(9 \%)$ had cytologic specimens, including a pleural fluid. Regarding metastatic site, there were $22(41 \%)$ liver, $20(37 \%)$ soft tissue, $8(15 \%)$ lung, $2(4 \%)$ gynecologic, $1(2 \%)$ bone, and $1(2 \%)$ brain. Six (11\%) were initially diagnosed with metastasis, $15(28 \%)$ were diagnosed concurrently, and $33(61 \%)$ patients were diagnosed with metastasis at a median interval of 18.5 mos (range 1-63 mos) after the primary CRC. Fifty-one (94\%) metastases showed intact expression of PMS2 and MSH6, and three (6\%) had loss of expression of either PMS2 or MSH6; IHC results on the metastases matched the primary tumor with $100 \%$ concordance.

Conclusions: We found $100 \%$ agreement for PMS2 and MSH6 protein expression in the metastasis with the MMR status (either PCR or IHC) of the primary in both tissue biopsies and cytologic specimens. Thus, our data support the use of metastatic tissue, including cytology, for assessment of MMR status, particularly those without available samples from their primary tumor. Up front preparation of unstained slides from cytology cell blocks may help ensure adequate material for future MMR IHC testing.

\section{Can Metastatic Lobular Carcinoma Be Reliably Distinguished} from Diffuse-Type Gastric Adenocarcinoma?

Lani K Clinton, Thomas Plesec, John R Goldblum, Kaveh Hajifathalian, Deepa T Patil. Cleveland Clinic, Cleveland, $\mathrm{OH}$.

Background: Metastatic invasive lobular carcinoma (mILC) to the GI tract is a clinical and pathologic mimic of primary diffuse-type gastric adenocarcinoma (PDGA). Although ER and GATA3 are highly sensitive and specific for ILC, we have seen anecdotal cases that lack ER/GATA3 expression. Given the clinical impact of this distinction, especially in the absence of relevant history, we studied clinicopathologic features of a large cohort mILC to the GI tract compared to PDGA. We assessed interobserver agreement among pathologists to apply select histologic features to differentiate these entities.

Design: We reviewed $29 \mathrm{mILCs}(62 \% \mathrm{bx})$ and 44 PDGAs (98\% bx)(1992-2016) for cytology [epithelioid (non-foamy) or globoid (foamy cytoplasm], architecture (single file, nests or anastomosing cords), and nuclear pleomorphism (none/minimal-2-3x lymphocyte or pleomorphic $>3-4 \mathrm{x}$ lymphocyte). Those with intestinalized gastritis and gland formation were excluded. Endoscopic findings and breast cancer history were recorded. ER, GATA3, and SATB2 was done on all cases. Interobserver agreement by 3 pathologists using aforementioend features to differentiate mILC and PDGA was measured using $\kappa$ statistic. All comparative tests were 2 -sided.

Results: Among 29 mILCs, 27 (93\%) had history of ILC, while GI bx was the initial diagnosis in 2 . Diffuse thickening, ulcer, and nodule/mass was significantly more common in PDGAs $(20 \%, 27 \%, 43 \%$ vs $7 \%, 10 \%, 10 \%$; p <.001); erythema narrowing was significantly common in $\operatorname{mILC}(38 \%$ vs $9 \%, \mathrm{p}<.001)$. mILCs were more often epithelioid $(100 \%$ vs $39 \%, \mathrm{p}<.001)$ with no/minimal pleomorphism $(79 \%$ vs $30 \%, \mathrm{p}<.001)$, and single file pattern $(62 \%$ vs $2 \%, \mathrm{p}<0.001)$; PDGAs were more globoid $(61 \%$ vs $0 \%, \mathrm{p}<.001)$, pleomorphic $(70 \%$ vs $21 \%, \mathrm{p}<.001)$, nested $(68 \%$ vs $38 \%, \mathrm{p}<.05)$, and anastomosing ( $30 \%$ vs $0 \%, \mathrm{p}<.01)$. Using these cytoarchitectural features, interobserver agreement ( 3 pathologists) to differentiate mILC from PDGA was very good $(\kappa=.77)$. SATB2 was positive in $13(30 \%)$ PDGAs and $0 \mathrm{mILCs}(\mathrm{p}<.01)$. GATA 3 and ER were $100 \%$ specific, and sensitivities were $83 \%$ and $72 \%$, respectively; PDGAs were negative $(p<.01) .5(17 \%)$ GATA3/ER negative mILCs were correctly identified by 3 observers using these histologic criteria.

Conclusions: Monotonous, epithelioid cells, arranged in a single file are significantly associated with mILC, whereas globoid (foamy cytoplasm) morphology, nuclear pleomorphism, and an anastomosing pattern are characteristic of PDGA. These histologic features can be extremely helpful in small biopsies that lack ER and GATA3 expression; SATB2 has limited sensitivity but high specificity for PDGA.

665 Potential Biomarkers to Delineate Tumors of Pancreatic Origin Ryan Coates, Valerie Cortright, Jeannette Mitchell, Abiy Ambaye, Michelle X Yang. University of Vermont Medical Center, Burlington, VT.

Background: Clinical diagnosis of pancreatic origin of tumors including adenocarcinoma, neuroendocrine tumor and others remains challenging in both biopsy and cytology specimens. Immunohistochemistry plays a key role in rendering a definitive diagnosis. Exploring tissue specific biomarkers for the diagnosis of pancreatic tumors remains significantly important. With these challenges in surgical pathology, we decided to search for pancreatic specific factors involving in pancreatic organogenesis and identified three key pancreatic lineage-specific transcriptional factors (TF) critical for pancreas development, including NKX2.2, PTF1A and PDX1. We investigated the diagnostic value of these TFs in pancreatic tumors.

Design: A total of 83 tumors from resection or biopsy at our institute were retrospectively retrieved from the archived blocks from January 2010 to December 2013. Tissue microarrays with $2-\mathrm{mm}$ core were made from following tumors, including 33 pancreatic ductal adenocarcinomas (PDAC); 14 well-differentiated neuroendocrine tumors (NET) from the stomach (2), duodenum (1), ileum (1), appendix (1), colon (1), rectum (1), pancreas (5), lung (1); liver metastatic NET (1); and 13 carcinomas from lung adenocarcinoma (1), lung squamous carcinoma (1), breast ductal (4), ovary serous (1), bladder urothelial (1), and prostate (1). All TMA cores were duplicated, except for a biopsy from the rectum. This pilot study is approved by the IRB (\#CHRMS 17-0059). Results: All three TFs were strongly expressed in the nucleus of normal pancreatic islet cells as internal positive controls. None of the PDACs or other carcinomas were positive for PDX1 expression. PPF1A was less specific and was positive in many non-pancreatic cancers of origin with variable intensity. Interestingly, NKX2.2 was strongly positive in all pancreatic NETs, although some of them were negative for both chromogranin or synaptophysin. Interestingly, NKX2.2 was also positive in all NETs in the lower gastrointestinal (GI) tract, but negative in upper GI such as gastric and all other NETs of non-GI organs. One liver metastatic NET was also positive for NKX2.2. Conclusions: NKX2.2 is a potential biomarker for NET of pancreatic and lower GI tract origin, especially for the origin of liver metastatic NETs and when other neuroendocrine markers are negative. We understand the limitations of this pilot study and more followup cases will be necessary for final conclusions.

666 Significance of Positive and Inhibitory Regulators in the TGF- $\beta$ Signaling Pathway in Colorectal Cancers

Ryan Coates, Juli-Anne Gardner, Valerie Cortright, Jeannette Mitchell, Takamaru Ashikaga, Joan Skelly, Michelle X Yang. University of Vermont Medical Center, Burlington, VT.

Background: TGF- $\beta$ signaling pathway is among the most common molecular changes in colorectal cancer (CRC). High levels of TGF- $\beta 1$ in $C R C$ were associated with poor clinical outcome and high-risk of relapse. Combined prevalence of SMAD4, SMAD2, and SMAD 3 mutations were seen in up to $50 \% \mathrm{CRCs}$. Little is known about the critical inhibitory regulator in the TGF- $\beta$ signaling pathway in CRC: PPM1A, aka protein phosphatase $2 \mathrm{C}$ alpha, a phosphatase involved in inhibition of the TGF- $\beta$ signaling pathway. Overexpression of PPM1A activates TP53, leading to cell cycle arrest and apoptosis, indicating a tumor suppressive role. We set out to understand the function of PPM1A and its relationship to Smad4 and TGF- $\beta$ in CRC

Design: A total of 177 surgically resected primary CRCs were retrospectively collected from January 2007 to December 2010. All pathologic stages with follow-up time of $\geq$ 5 years are included. IHC for PPM1A, Smad4, and TGF- $\beta 1$ were performed on 2-mm core the tissue microarrays. Nuclear stains on IHC were graded using a 3-tier method for SMAD4 based on intensity: strong (2), moderate (1) and weak/negative (0); and a 2 -tier system for PPM1A and TGF- $\beta$ : strong (1) and weak/negative (0). All statistical analyses were conducted using the Systat software (version 11). Overall survival for each subgroup was examined using Cox Proportional Hazard function models and Kaplan Meier plots.

Results: PPM1A and Smad4 protein expression is positively correlated to TGF- $\beta$ expression, but PPM1A is not correlated with Smad4. . Weak or loss of Smad4, PPM1A and TGF- $\beta$ was observed in 67,77 , and 8 cases, respectively. Strong SMAD4 expression was associated with tumor infiltrating lymphocytes $(\mathrm{p}=0.016)$ and presence of precursor adenoma $(\mathrm{p}=0.017)$. Loss of SMAD4 expression correlates weakly to metastasis $(\mathrm{p}=0.07)$ and perineural invasion $(\mathrm{p}=0.09)$. PPM1 A and TGF- $\beta$ expression is not correlated to any of the clinicopathological parameters. A strong Smad4 expression was significantly associated with better overall survival. High levels of nuclear PPM1A and TGF- $\beta$ also showed better survival tendency, although the $\mathrm{p}$ value was borderline ( 0.067 and 0.16 , respectively).

Conclusions: To our knowledge, this is the first study to demonstrate protein expression patterns of three important proteins in the TGF- $\beta$ signaling pathway in colorectal cancers and their correlations to clinicopathological parameters and overall survival. Loss of inhibitory regulators in the TGF- $\beta$ signaling pathway such as loss of PPM1A and loss of TGF- $\beta$ appears to be additional important molecular events in colorectal cancers.

667 "Rapid Progression" to High-Grade Dysplasia or Adenocarcinoma in Barrett's Esophagus Is Associated with Prevalent or Missed Neoplasia at Index Endoscopy

Aaron J Cohen, Amitabh Srivastava. Brigham and Women's Hospital, Boston, MA. Background: Barrett's esophagus (BE) is a premalignant condition that confers an increased risk of progression to esophageal adenocarcinoma (EAC). Rarely, carcinoma develops in BE patients within 1-2 years of their index endoscopy. It is unclear whether this represents prevalent neoplasia missed on initial endoscopy or rapidly progressive incident neoplasia. We examined clinical, endoscopic, and pathological factors associated with a diagnosis of high grade dysplasia (HGD) or EAC within one year of $\mathrm{BE}$ diagnosis.

Design: A retrospective analysis of patients diagnosed with BE from 1989-2015 was performed. Progressors were defined as patients diagnosed as BE without dysplasia, indefinite for dysplasia or low grade dysplasia (LGD) on initial exam, who were subsequently diagnosed with HGD or EAC. An age, gender and BE length matched group of non-progressors was also identified and defined as BE patients who never had a diagnosis of HGD or EAC on initial or follow up exams. Chart review was performed for demographic data, endoscopic findings including $B E$ length and number of biopsies obtained, and pathologic diagnosis at each examination

Results: A total of 48 progressors (28 EAC; 20 HGD) were identified with an average time to progression of $6.04 \mathrm{yrs}$ (range $0.1-19.75 \mathrm{yrs}$ ). Of these, 8 progressed in $<1$ year $(16.7 \%)$, and 15 in $<2$ years $(31.2 \%)$. The average age the time of BE diagnosis was $57.0 \mathrm{yrs}, 43(89.6 \%)$ were male, and the average BMI at the time of diagnosis was 27.5 . $9 / 48$ progressors had LGD diagnosis at index exam. Although patients with rapid $(<1$ year) progression had higher rate of LGD at index examination $(50 \%$ vs $12.5 \%$, $\mathrm{p}=$ $0.09)$ and a longer BE segment length $(6.8 \mathrm{~cm}$ vs $5.2 \mathrm{~cm}, \mathrm{p}=0.47)$ neither of the two parameters were statistically significant. When patients with LGD at initial diagnosis were removed from analysis only $4 / 39(10.2 \%)$ patients remained who progressed to $\mathrm{HGD}$ or EAC in $<1$ year. The mean BE segment length in these four patients was $8.0 \mathrm{~cm}$ (compared to $4.81 \mathrm{~cm}$ for the remaining progressors). An average of only 10.7 biopsies per patient were obtained in three of four patients in this group and no information regarding biopsy protocol was available in one patient.

Conclusions: Rapid progression ( $<1$ year) was associated with a longer BE length and LGD suggesting prevalent dysplasia at index examination. Only 4 rapid progressors without LGD on initial endoscopy were identified and appear to have been suboptimally biopsied raising the possibility of missed neoplasia. These findings need to be validated in future studies. 
668 HER2/ ERBB2 Amplification in Colorectal Carcinoma Is Associated with KRAS Wild Type Status, Microsatellite Stability, and Left Sided Tumors

Paolo Cotzia, Deepu Alex, Sumit Middha, Jaclyn Hechtman, Sandy Liu, Deepthi Rao, Efsevia Vakiani, David S Klimstra, Jinru Shia. Memorial Sloan Kettering Cancer Center, New York, NY.

Background: HER2 amplification has been detected in a subset of colorectal carcinoma (CRC), and is potentially a novel target for selected patients. Here, we investigate the clinicopathological and molecular features of a series of HER2 amplified CRC (HCRC) and explore potential morphological and molecular characteristics of this subset.

Design: A total of 1170 cases of advanced CRC and molecular data from a 410 gene hybrid capture next generation sequencing assay were retrospectively analyzed for presence or absence of HER2 amplification (HCRC and NHCRC respectively), clinical and histologic features, and molecular features including RAS and TP53 mutation as well as microsatellite instability (MSI) status. Primary site was classified as right (cecum to transverse colon) or left (descending to rectum). HER2 amplification was defined by a $\log 2$ ratio $\geq 1$ of HER2 copies in tumor compared to that in matched normal.

Results: 33 patients harbored HER 2 amplification $(2.8 \%$ of all CRC) [male $=25$, female $=8]$ with a median age of 51 years (range: 30-72). Histologic features included moderate differentiation $(\mathrm{n}=24)$, poorly differentiation $(\mathrm{n}=8)$, and micropapillary histology $(\mathrm{n}=1)$. HCRC was predominantly left sided $(76 \%)$. The most frequently mutated gene of the HCRC group was TP53 (82\%). In HCRC, the frequency of RTK pathway alterations was significantly lower $27.7 \%$ compared $63.8 \%$ in NHCRC $(\mathrm{p}<0.01)$. KRAS mutations occurred in $18 \%$ of HCRC compared to $44 \%$ of NHCRC $(\mathrm{p}<0.01)$. PIK3CA alterations were found in $6 \%$ of HCRC compared to $20 \%$ in NHCRC $(\mathrm{p}<0.05)$. All 33 HCRC were microsatellite stable (MSS).

Conclusions: HER2 amplifications occur in approximately $3 \%$ of advanced CRC. HCRC are most often left-sided, KRAS wild type, and MSS. This molecular subgroup of advanced CRC with less common downstream MAPK pathway mutations may benefit from anti-HER2 therapy.

\section{Acute Esophageal Necrosis (Black Esophagus). Detailed} Pathologic Description of Five Cases

Isabelle H Cui, Gerardo Aristi Urista, Rhonda K Yantiss, Erika Hissong, Steven Salvatore, Jose Jessurun. New York Presbyterian-Weill Cornell Medicine, New York, NY; Hospital General de México, Mexico City, Mexico.

Background: Acute esophageal necrosis (AEN) is a rare severe form of esophageal injury that develops in older patients with several comorbidities. It has a characteristic gross and endoscopic appearance with diffuse black discoloration of the esophageal mucosa. To date most cases have been reported in the clinical literature and the histologic features have not been well characterized. In this study we describe the histopathologic and immunohistochemical features of five cases of AEN to improve its recognition in biopsy material and to contribute to the understanding of its pathogenesis.

Design: The study group consisted of five patients evaluated at the time of autopsy (3) or biopsy review (2). The specimens were assessed for type of necrosis, distribution of inflammatory cells, vascular injury, thrombosis and pigment deposits. Cases were evaluated with Prussian blue stains and immunohistochemical stains that assess inflammatory cells (myeloperoxidase, CD20, CD3 and CD163), squamous epithelial cells (pancytokeratin and p40) and muscle (smooth muscle actin).

Results: Patients age, sex, clinical conditions and the type of specimen are summarized in the table. All specimens show complete mucosal necrosis with a distinct band of coagulative necrosis composed of crowded nuclei reminiscent of necrotic inflammatory / lymphoid cells. In this area and throughout the esophageal wall in autopsy specimens were abundant scattered CD163 positive macrophages. However most cells were negative for lymphoid, granulocytic and squamous epithelial markers. Prussian bluenegative intra and extravascular brown pigment deposits and intravascular thrombi were present.

\begin{tabular}{|c|c|c|c|}
\hline Age & Sex & Associated Conditions & $\begin{array}{c}\text { Diagnostic } \\
\text { specimen }\end{array}$ \\
\hline 70 & M & Advanced prostate cancer, duodenal ulcer, hypovolemic shock & Autopsy \\
\hline 43 & M & Diabetes mellitus, severe vomiting & Autopsy \\
\hline 55 & F & Severe vomiting, hypovolemic shock & Autopsy \\
\hline 55 & M & Alcoholic cirrhosis & Biopsy \\
\hline 66 & M & Alcoholic cirrhosis & Biopsy \\
\hline
\end{tabular}

Conclusions: Dense basophilic coagulative necrosis and Prussian blue-negative pigment deposits are pathognomonic histopathologic findings of AEN. Macrophage recruitment and intravascular thrombi may participate in its pathogenesis.

670 Ambiguous Adenoma Reporting Leads to Worse Patient Outcomes

Daniel N Dang, Suntrea TG Hammer. UT Southwestern, Dallas, TX.

Background: The American Gastroenterological Society has discrete colorectal surveillance screening guidelines with recommended surveillance intervals (SI) based on the number adenomatous polyps (AP) and the presence of high-risk features. Accurate pathologic reporting is essential in determining appropriate intervals. Incongruity often occurs between histologic tissue pieces and the endoscopic polyp number, leading to ambiguous phrasing like "fragments of tubular adenoma". We explore the effect of reporting style on SI and patient outcome.

Design: We identified cases of APs between 06/2008 and 03/2011 in the UTSW Pathology Database. We excluded cases with histories of poor bowel preparation, polyposis syndromes, inflammatory bowel disease, and invasive carcinomas. Unenumerated APs served as study cases and enumerated APs as controls. High-risk features were categorized as $\geq 3 \mathrm{APs}, \mathrm{APs} \geq 1 \mathrm{~cm}$, villous architecture, and high grade dysplasia. Patient demographics, endoscopic polyp number, SI, repeat endoscopic findings, gross exam findings, and final pathologic diagnosis were recorded. Diagnostic categories were then correlated with recommended SIs and patient outcomes. We calculated statistical significance with unpaired t-tests and the chi-squared test for proportions.

Results: From 955 cases, 298 were eligible (study: 147, Control: 151). The M:F ratio $(53 \%$ vs $56 \%, \mathrm{p}=0.60)$ and mean age ( 60.8 vs $60.1 \mathrm{yrs}, \mathrm{p}=0.43$ ) were not significantly different between study and controls, respectively.

The study group had 85 patients with $1-2 \mathrm{APs}, 48$ with $\geq 3 \mathrm{APs}$, and 20 with advanced APs, compared to 109,14 , and 26 in the control group, respectively.

SIs statistically differed between the 2 groups. Both had proper follow up in the 1-2 AP category ( 52.8 mos study vs 53.6 mos control, $p=0.74$ ). SIs in those with highrisk features were prolonged in the study group vs controls $(41.7 \mathrm{mos}$ vs $33.6 \mathrm{mos}$, $\mathrm{p}=0.012$ ). The interval in study patients with $\geq 3 \mathrm{APs}$ was significantly longer than suggested $(\mathrm{p}=0.0001)$

The incidence of advanced APs and $\geq 3$ APs was greater in the study group vs controls $(24.6 \%$ vs $2.6 \%, \mathrm{p}=0.0035)$ at follow up. The study group also had 3 interval adenocarcinomas.

Conclusions: Diagnostic reporting of APs has a significant impact on SI and patient outcome. Appropriate intervals were seen with polyp enumeration compared to ambiguous reporting. Ambiguous reporting led to significantly increased high-risk disease, including 3 interval adenocarcinomas. We recommend a clear enumeration of polyps in correlation with endoscopic findings whenever possible.

671 Invasive Carcinomas of the Ampulla with Deceptively Bland Patterns: An Analysis of 35 Cases

Drew Davis, Yue Xue, Michelle D Reid, Bahar Memis, Alyssa Krasinskas, Volkan Adsay. Emory University School of Medicine, Atlanta, GA.

Background: Due to the architectural and glandular complexity of the organ, distinguishing invasive carcinoma from pre-invasive neoplasms (with pagetoid extension into lobules) is a well-known challenge in the ampulla.

Design: 35 resected invasive ampullary carcinomas with deceptively benign or noninvasive appearance were identified and analyzed.

Results: Four distinct patterns were elucidated. 1. Villous adenoma-like pattern $(n=14)$ characterized by villiform organized papillae with adenomatous cytology and a lobular growth pattern that rendered them indistinguishable from a villous adenoma. 2. Mesonephroid adenocarcinoma $(n=14)$ composed of small, round, well-formed tubular glands with cuboidal cells and intraluminal eosinophilic material which mimic mesonephric remnants. 3. Compact blunt invasion $(n=6)$ with lobulated groups of rounded glands showing "pushing-border" invasion and lacking a stromal response. Some of these cases demonstrated a papillary pattern. 4. Adenoma-malignum pattern $(n=1)$ forming extremely well-differentiated tubules that resemble normal endocervical glands. Demographic information was available for 23 cases and revealed no significant differences in age, sex, pathologic stage or survival between the four patterns.

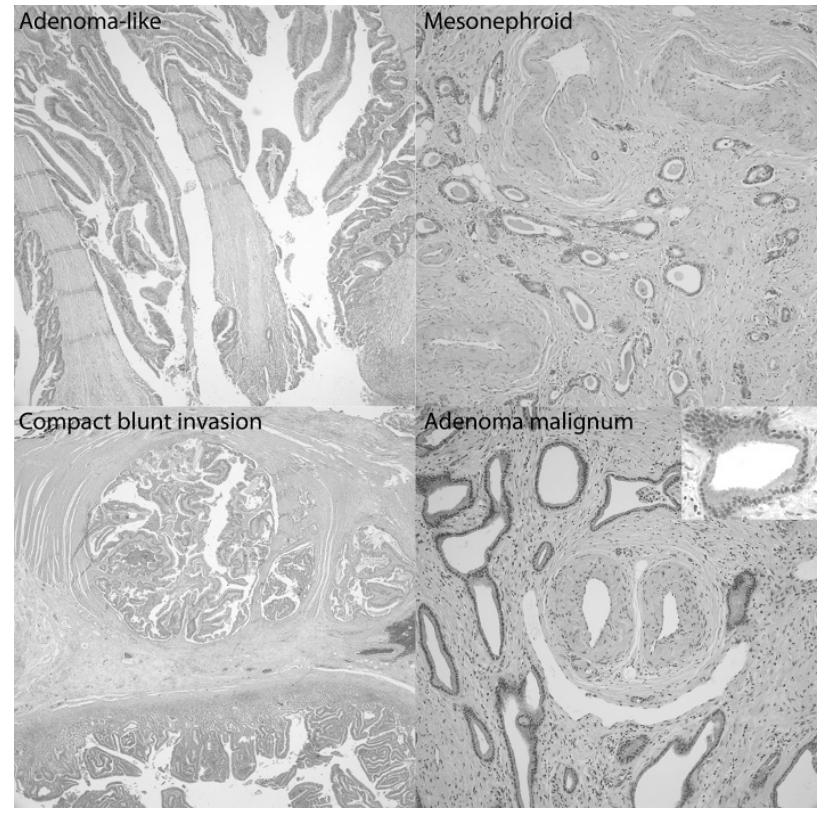

Conclusions: It is important to recognize these deceptive patterns of invasive carcinoma in ampullary carcinomas because they are highly prone to misdiagnosis, especially in biopsies. Some of these peculiar patterns may be the result of specific molecular alterations, as has been shown for the adenoma-like invasive colorectal carcinomas (Gonzalez, PMCID: PMC4619179) and as such may warrant further study. 
672 Assessing and Reporting Tumor Budding in Colorectal Cancer: Recommendations Based on the International Tumor Budding Consensus Conference (ITBCC 2016)

Heather Dawson, Alessandro Lugli, Richard Kirsch, Inti Zlobec, Robert H Riddell, Gieri Cathomas, Fred Bosman, Philip Quirke. Institute of Pathology, University of Bern, Bern, Switzerland; University of Toronto, Toronto, Canada; Institute of Pathology, Kantonsspital Liestal, Liestal, Switzerland; University Institute of Pathology, Lausanne University Medical Center, Lausanne, Switzerland; Leeds Institute of Cancer and Pathology, University of Leeds, Leeds, United Kingdom.

Background: Tumor budding has been consistently shown to be an independent prognostic factor in colorectal cancer (CRC). However, its implementation in daily routine and integration in guidelines have been held back largely due to the lack of a standardized scoring method. Therefore, the aim of the international tumor budding consensus conference (ITBCC) was to agree on a scoring system for tumor budding in CRC.

Design: ITBCC was held in April 2016 in Bern, Switzerland. The consensus group consisted of 23 voting members from eleven different countries with expertise in tumor budding. Prior to the meeting, a systematic search of the literature was performed, forming the basis of the initial statements proposed by the steering committee. The Grading of Recommendation Assessment, Development and Evaluation (GRADE) approach was used to assess the strength of recommendation and quality of evidence. The statements were discussed in 9 sessions, finalized and voted on by the participants. Results: Consensus statements include the following:

- A tumor bud is defined as a single tumor cell or a cell cluster consisting of 4 cells or less. - Tumor budding is an independent predictor of lymph node metastases in CRC and an independent predictor of survival in Stage II CRC.

- Intratumoral budding exists in CRC and has been shown to be related to lymph node metastases.

- Tumor budding and grade are not the same.

- Tumor budding is counted on H\&E and assessed in the hotspot $\left(0.785 \mathrm{~mm}^{2}\right)$ at the invasive front. A 3-tier system should be reported together with the budding count.

- Tumor budding should be taken into account with other clinico-pathological features in a multidisciplinary setting.

- Tumor budding should be included in guidelines for CRC reporting.

Conclusions: Tumor budding is an independent prognostic parameter in CRC, strongly affects the management of stage I and stage II CRC patients and should be therefore included in CRC reporting, guidelines and staging systems.

\section{Tumor Budding Assessed by the International Tumor Budding Consensus Conference (ITBCC) Recommendations Is a Strong Predictor} of Disease-Free Survival in Stage II Colorectal Cancer

Heather Dawson, Naziheh Assarzadegan, Richard Kirsch, Robert H Riddell, Inti Zlobec, Alessandro Lugli. Institute of Pathology, University of Bern, Bern, Switzerland; University of Florida, Gainesville, FL; Mount Sinai Hospital, University of Toronto, Toronto, Canada.

Background: Tumor budding has been shown to be a strong and independent prognostic factor in colorectal cancer (CRC) and has the potential to guide management in Stage I and Stage II tumors. The International Tumor Budding Consensus Conference (ITBCC), held in April 2016 in Bern, Switzerland, agreed on a set of recommendations for assessing and reporting tumor budding in CRC. The aim of this study was to validate the method proposed by ITBCC in the clinically relevant scenario of Stage II CRC.

Design: 151 Stage II CRC patients were included in this study. Budding was assessed on scanned H\&E-stained slides in a single hot spot measuring $0.785 \mathrm{~mm}^{2}$. Cutoffs as defined by ITBCC were used: Low (Bd1): 0-4 buds, intermediate (Bd2): 5-9 buds, high (Bd3): $\geq 10$ buds. Associations with clinicopathological features, disease free survival (DFS) and overall survival (OS) were examined.

Results: The average number of buds/hotspot was 6.8 (median 5.0, min 0, max 36). $43.1 \%$ of cases were $\mathrm{Bd} 1,27.2 \% \mathrm{Bd} 2$ and $29.8 \% \mathrm{Bd} 3$. As a continuous variable, tumor budding was associated with extramural venous invasion (EMVI) $(\mathrm{p}=0.05)$. Tumor budding was associated with poorer OS in univariate analysis $(\mathrm{p}=0.0386$, HR 1.048 , $95 \%$ CI 1.002-1.095). For 3- and 5- year DFS, Bd3 was associated with worse survival in comparison with $\mathrm{Bd} 1 / 2$ ( $\mathrm{p}=0.0031$ and $\mathrm{p}=0.0025$, respectively). Tumor budding retained its prognostic effects in multivariate analysis for DFS adjusting for $\mathrm{pT}$, tumor grade and EMVI ( $\mathrm{p}=0.006, \mathrm{HR} 3.293,95 \% \mathrm{CI} 1.66-6.53)$.

Conclusions: This study provides promising data on tumor budding assessed by the ITBCC method in Stage II CRC patients. Especially Bd3 shows a detrimental adverse impact on DFS in comparison to $\mathrm{Bd} 1 / \mathrm{Bd} 2$. Based on the ITBCC statements tumor budding should be included in reporting guidelines and staging systems in CRC.

674 Co-p53/Ki-67 Immunostaining and NGS Molecular Analysis as an Efficient Approach to Detect Mutant p53 and IBD-Associated Dysplasia Audrey Deeken-Draisey, Haonan Li, Xiaoming You, Nike T Beaubier, Sambasiva Rao, Jie Liao, Guang-Yu Yang. Northwestern, Chicago, IL.

Background: It is challenging to distinguish reactive epithelium from low and high grade dysplasia in chronic active inflammation in IBD. Analysis of genomic mutations has identified $p 53$ missense point mutation as an early driver mutation in IBD-associated colorectal carcinogenesis and results in its protein accumulation in nuclei. Active inflammation and oxidative stress induce wild type p53 protein expression and there are no well-established criteria to distinguish mutant $\mathrm{p} 53$ from wild type immunohistochemically (IHC). This study aims to identify clonal dysplastic epithelium in IBD with co-p53/Ki-67 IHC and next generation sequencing (NGS) to detect mutant $p 53$.
Design: 66 IBD cases were identified: 35 low-grade, 7 high-grade, 3 indefinite dysplasia, and 21 IBD without dysplasia. 38 colon adenocarcinomas were included for this study (18 with $p 53$ missense mutation and 20 with no $p 53$ mutation). IHC for $\mathrm{p} 53$ and Ki-67 was performed on selected slides from each case with appropriate controls. Nuclear positivity of p53 and Ki-67 in epithelial cells/lesional tissue from 5 high power fields was analyzed. P53 positive staining was calculated as positive staining cells $<,=$, or $>$ Ki-67 proliferative cells. With NGS approach, 553 mutation was performed for IHC positive dysplasia.

Results: With Co-p53/Ki-67 IHC, all 18 adenocarcinoma with p53 mutation displayed strong/intense $\mathrm{p} 53$ staining and was $\geq \mathrm{Ki}-67$ labeled cells, while all 20 adenocarcinoma without $\mathrm{p} 53$ mutation exhibited no/scattered p53 positive cells and p53 was much lower than Ki-67 labeled cells. The 21 IBD without dysplasia and 3 indefinite showed scattered $\mathrm{p} 53$ positive cells (Fig.A). Strong/intense p 53 staining was present in $75 \%$ of low-grade (26/35, Fig.C) and all high-grade dysplasia (7/7, Fig.E). The ratio of p53:ki-67 positive cells was $<1$ in non-dysplastic IBD (Fig.B), $\geq 1$ in low-grade (Fig.D) and high-grade dysplasia (Fig.F). p53 missense mutation was confirmed in these dysplastic lesions with NGS.
P53
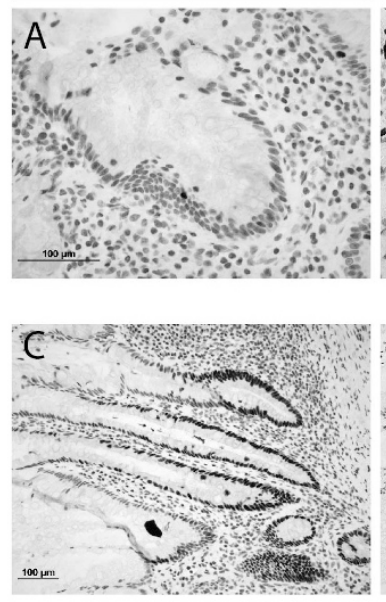
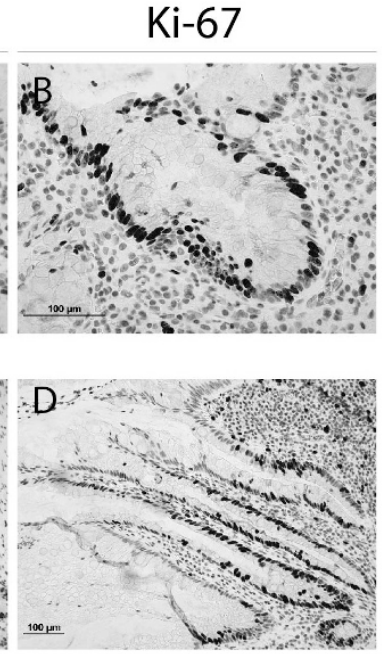

1000

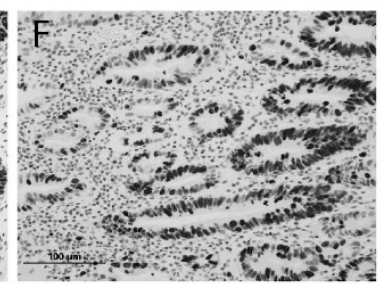

Conclusions: Our results demonstrate that 1) co-p53/Ki-67 IHC using criteria of p53 positive cells $\geq \mathrm{Ki}-67$ labeled cells is an efficient approach to detect mutant $\mathrm{p} 53$ and is further confirmed by NGS; and 2) co-p53/Ki-67 IHC is a practical approach (sensitive and specific) to detect IBD-associated dysplasia.

675 Downregulation of Friend Leukemia Integration-1 (FLI1) Characterizes the Stepwise Progression from Normal Stomach Glands to Gastric Adenocarcinomas

Armando Del Portillo, Aqiba Bokhari, Anne Koehne de Gonzalez, Elena V Komissarova, Helen E Remotti, Jorge Sepulveda, Antonia Sepulveda. Columbia University, New York, NY.

Background: Gastric adenocarcinoma (GC) is the 5th most common cancer worldwide but is the 3rd leading cause of cancer death. FLI1 is an ETS family transcription factor that regulates genes involved in proliferation and differentiation. FLI1 is implicated in tumorigenesis, such as in Ewing's sarcoma where a translocation creates an EWS-FLI1 oncogenic fusion protein. However, few studies have examined the role of FLI1 in epithelial cancers. In human breast cancer cells, overexpression of $F L I I$ led to inhibition of apoptosis, thereby promoting survival and malignant potential. In a murine breast cancer model, downregulation of $F L I I$ promoted breast carcinogenesis. In human GCs, we recently reported that $F L I I$ expression is inversely correlated with $\mathrm{CpG}$ methylation levels in GCs, suggesting a tumor suppressor role. However, the distribution of FLII expression in the normal stomach and a potential association with clinicopathological features of GC have not been reported.

Design: To examine FLI1 in situ, we analyzed 8 normal stomach controls (antral and oxyntic mucosa), 8 stomachs with intestinal metaplasia (IM) and 91 human GCs by immunohistochemistry (IHC) for FLI1 (clone MRQ-1, Cell Marque) on formalin-fixed paraffin embedded tissue. Tissue microarrays were scored for nuclear intensity $(0,1$ or 2) and for percentage of tumor cells at each intensity. To capture the variability of a tumor, a composite FLI1 IHC score was calculated by summing the frequency of cells for each intensity score x 100, with a maximum possible score of 200. Correlation analyses were determined between tumor clinicopathologic features (tumor stage and subtype) and FLII expression. 
Results: Normal antral and oxyntic mucosa and IM showed strong nuclear staining at similar levels to internal positive controls (endothelial cells and lymphocytes). Most GCs showed weak staining with an overall lower composite score as compared to normal ( $\mathrm{P}$ $<1 \mathrm{x} 10 \mathrm{e}-17)$ and IM $(\mathrm{P}<1 \times 10 \mathrm{e}-26)$. Normal gastric glandular tissue showed an average score of 187.9, IM an average of 193.7, and GC an average of 108.2. No significant difference in FLII expression was seen between tumor subtypes or tumor stage.

Conclusions: FLII expression is significantly decreased in human GCs as compared to normal stomach glandular tissue and gastric IM. Our findings suggest that FLII downregulation may play a role in GC development and progression and support a role for FLII as a tumor suppressor gene.

676

Loss of MAdCAM-1 Expression in Colorectal Adenocarcinoma Kelly Devereaux, Adam Gomez, Sushama Varma, Reetesh Pai, Nir Modiano, Teri Longacre, Rob West, Megan Troxell. Stanford Health Care, Stanford, CA; University of Pittsburgh School of Medicine, Pittsburgh, PA; Oregon Health \& Science University, Portland, OR.

Background: Decreased tumor-infiltrating lymphocytes (TILs) in colorectal carcinoma (CRC) is associated with a poorer overall and recurrence-free survival; however, the mechanisms underlying immune evasion and reduced TIL trafficking and recruitment are not yet well characterized. Lymphocytes expressing the alpha4beta7 integrin specifically home to gastrointestinal mucosal tissue via their interaction with Mucosal Addressin Cell Adhesion Molecule-1 (MAdCAM-1), a spatially restricted addressin which has been shown to be overexpressed in inflammatory bowel disease (IBD). Little is known about this pathway of lymphocyte recruitment in CRC.

Design: Tissue microarrays were constructed with over 200 patient samples of $\mathrm{CRC}$, the majority of which have normal, matched controls. Immunohistochemistry for MAdCAM-1 was performed and scored as positive or negative staining of the endothelium of vessels within normal colonic mucosa or CRC.

Results: Of the 206 colorectal carcinoma cases with scoreable matched normal colonic mucosa controls, $92.2 \%(190 / 206)$ of the CRC show loss of MAdCAM-1 within the vessel endothelium, whereas only small minority of $7.8 \%(16 / 209)$ show retained expression (Table 1). Interestingly, the majority of the CRC cases that show MAdCAM-1 expression demonstrate at least focal mucinous features. Additionally, out of $3 \mathrm{CRC}$ from patients with inflammatory bowel disease, MadCAM-1 expression is negative in 2 cancers, and positive in 1 superficially invasive case (full sections stained).

\begin{tabular}{|c|c|c|c|}
\hline MadCAM-1 expression & Normal + & Normal - & Total $\mathrm{n}=$ \\
\hline Tumor + & $15(7.3 \%)$ & $1(0.5 \%)$ & $16(7.8 \%)$ \\
\hline Tumor - & $141(68.4 \%)$ & $49(23.8 \%)$ & $190(92.2 \%)$ \\
\hline Total $\mathrm{n}=$ & $156(75.7 \%)$ & $50(24.3 \%)$ & $=206$ \\
\hline
\end{tabular}

Conclusions: In this study, we demonstrate a striking loss of MAdCAM-1 expression within the endothelium in $92.2 \%(190 / 206)$ of CRC. Future studies will help elucidate whether loss of MAdCAM-1 expression in colorectal cancer may be one of the mechanisms of immune modulation in GI tract carcinogenesis. Given that novel monoclonal antibodies targeting the alpha4beta 7 integrin have recently been approved to treat refractory IBD, the role of MAdCAM-1 in the tumor microenvironment and/or $\mathrm{CRC}$ will be important for treated patients.

\section{Applications of Phosphohistone H3 and Ki-67 by Immunohistochemistry in Aiding Mitotic Counting in Gastrointestinal Stromal Tumors (GISTs)}

Yanli Ding, Qing Zhao. Brigham and Women's Hospital, Boston, MA; Boston University Boston Medical Center, Boston, MA.

Background: GIST is one of the most common mesenchymal tumors involving GI tract. The latest criteria for tumor risk prediction depends on tumor location, size and proliferating index rate (PIR) measured by mitotic count (MC). Accurately measurement of tumor PIR plays critical role in tumor grading and management. It is required to count 50 high power fields (HPFs) on selected hot spots on hemotoxylin and eosin (H\&E) slides. Low yield of reproducibility and great intraobserver variability had always been a challenge. We proposed to apply immunostains with phosphohistone H3 (PPH3), a marker for detecting only those mitotically active cells as they are recognized on H\&E. Ki-67 will be used for checking the overall PIR using image-assisted analysis (IAA). Design: Total of 34 resection specimens were retrospectively reviewed. 24 cases were from stomach, 9 from small bowel and 1 from colon. Patients' median age is 65 (37 to 85$)$. Female:male ratio is 0.65 . Tumor average size is $5.3 \mathrm{~cm}(0.5 \mathrm{~cm}$ to $16 \mathrm{~cm}) .21$ $(67 \%)$ GIST tumors showed spindled, $2(3 \%)$ showed epitheloid and $10(29 \%)$ showed mixed histology. The numbers of positive cells highlighted by PPH3 immunostain were counted manually in $50 \mathrm{HPFs}$. These results were compared in parallel to MCs on H\&E slides. Immunostains for $\mathrm{Ki}-67$ analyzed by IAA were used for measuring the overall PIR. Correlation coefficient analysis and student t-test for p-value were analyzed.

Results: 23 (out of 34,68\%) cases were low risk tumors, 11 (out of $34,32 \%$ ) were in high risk group. The MCs range from 0 to 58 (per $50 \mathrm{HPFs).} \mathrm{The} \mathrm{PIR} \mathrm{measured} \mathrm{by} \mathrm{Ki-67}$ immunostain using IAA showed a range of $1 \%$ to $25 \% .33$ out of 34 cases show positive nuclear staining for $\mathrm{PPH} 3$, with unique nuclear contours that can be easily recognized by IHC, ranged from 0 to 100 . All low risk group tumors showed $0-5$ mitotic figures, $0 \%$ to $11 \%$ PIR by Ki-67 and 0 to 10 mitotic counts by PPH3. While the high risk tumors have a cutoff of $>10 \%$ (range of $10 \%$ to $25 \%$ ) PIR by Ki-67, $>12$ mitotic count (range of 12 to 100) by PPH3. There is a strong correlation $(\mathrm{r}=0.81, \mathrm{P}<0.05)$ between PIR measured by Ki-67 and MCs on H\&E stain. PHH3 IHC assisted-MC and actual $\mathrm{MC}$ showed a significantly strong correlation $(\mathrm{r}=0.78, \mathrm{P}<0.05)$.
Conclusions: Our study indicated that application of immunostains with Ki-67 and PPH3 displayed great values for accurately monitoring the mitotic count. More cases are needed for conclusive cutoffs from multiple institution in the future.

$678 \quad$ Brushing Cytology with Adjunctive FISH and Biomarker Analyses Is Highly Sensitive and Specific in the Early Detection of LowGrade Dysplasia in Barrett's Esophagus

Yimin Dong, Jing Zhai, Daniel Megna, Ashish Prasad, Sam Moussa, David Baunoch, Anil Prasad. College of Medicine, University of Arizona, Tucson, AZ; Northwest Medical Center, Tucson, AZ; College of Public Health, University of Arizona, Tucson, AZ; Endo Associates of Staten Island, Staten Island, NY; Foothill High School, Tucson, AZ; Adobe Gastroenterology, PLLC., Tucson, AZ; Pathnostics, Inc., Irvine, CA.

Background: Four-quadrant biopsies are utilized to evaluate presece of dysplasia in Barrett's esophagus (BE). Studies suggest that brushing cytology analysis seems superior to traditional biopsy interpretation based on sampling error reduction, better patient compliance and less time consumption. In this study, we explore the genomic alterations and protein expression profiles on brushing cytology samples of BE patients. Compared to biopsy, cytologic assessment along with molecular genomic assays, DNA ploidy analysis and protein expression profiles maybe more reliable in the detection of low-grade dysplasia (LGD) in BE, and provide additional objective methods in the surveillance of BE patients.

Design: 96 patients visited two GI clinics from 5/01/2015 to 8/31/2016. Results of brushing cytology were compared with concurrent biopsy samples. Immunohistochemical (IHC) analyses (Ki-67, p53 and AMACR) and FISH assays to detect genomic alterations (ERBB2 at 17q12, p16 at 9p21, ZNF217 at 20q13, and cMYC at 8q24) were applied on the cytology specimens. Statistical analysis $\left(\mathrm{Chi}^{2}\right.$ test, logistic regression \& prediction models) was performed.

Results: 81 of 96 cases had concurrent cytology and histopathology results. 30 cases were diagnosed as LGD by cytology and biopsy showed LGD in 21 cases. In comparison to BE without dysplasia, LGD cytology specimens showed aneuploidy ( $95 \%$ vs. $7.5 \%$ ), loss of p16 gene (57\% vs. $11 \%)$ high Ki-67 (90\% vs. $12 \%)$ and p53 mutation $(91 \%$ vs. $12 \%) .9$ cases were positive for LGD on cytology but negative on biopsy, among which 8 cases had p16 gene loss with p53 mutation.

Conclusions: BE brushing cytology is sensitive (91\%) and specific (87\%) in detecting LGD. Loss of p 16 gene and 553 mutation are common in BE-LGD. Chromosomal gains of ERBB2, CMYC, ZNF217 and AMACR protein expression do not correlate with LGD. In comparison to histopathology and cytology, loss of p16 and p53 mutation corroborated with cytology. This consistency between cytomorphology and $\mathrm{p} 16$ gene loss may suggest their early predictive roles in progression of $\mathrm{BE}$ dysplasia.

\section{Lateral Spread in Esophageal Squamous Cell Neoplasia}

Ogechukwu Pearl Eze, Yaman Tarabishy, Kevin Waters, Maryam Pezhouh, Lothar Veits, Andrea May, Christian Ell, Elizabeth A Montgomery, Michael Vieth. Johns Hopkins Hospital, Baltimore, MD; Klinikum Bayreuth, Bayreuth, Germany.

Background: Low grade squamous dysplasia (LGD) is defined as dysplasia of basal third with slight cellular pleomorphism and preserved stratification. Epithelial changes confined to the basal layer with marked nuclear pleomorphism and hyperchromasia exceeding that expected in LGD are reported as lateral spread of squamous cell neoplasia but not as LGD in Japan. Most esophageal squamous carcinomas (SCC) show intraepithelial spread of carcinoma (cancerization of adjoining squamous epithelium) for $\geq 2 \mathrm{~mm}$, confined to the basal layer or involving the entire epithelial thickness. Clinical significance of lateral spread of intraepithelial squamous neoplasia is unknown.

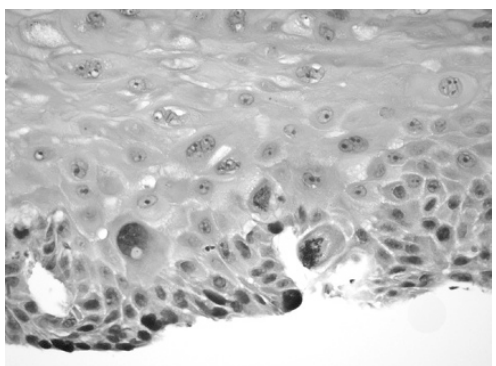

Design: Materials from 96 patients with lateral spread of squamous cell neoplasia confined to the basal/parabasal cell layer from 2 institutions from 2007 to 2016 were retrospectively reviewed.

Results: There was follow-up for 66 of the 96 cases (range; 1-63 months). There was a male predominance (M:F-3:1) and a mean age of $66.6 \pm 9.11$ years. Synchronous invasive SCC was detected in 41 patients and 25 had biopsy diagnosis of lateral spread only. 4 patients had prior or subsequent diagnoses of invasive SCC. 1 patient had a prior diagnosis of lateral spread, 5 with SCC in situ (Tis) and 9 with invasive SCC (T1a:8, $\mathrm{T} 2: 1$ ), 18 patients were shown to have invasive SCC after a biopsy diagnosis of lateral spread, with 5 showing persisting lateral spread. Following endoscopic resection, 8 patients had Tis, 3 had T1a and 1 had T1b carcinoma, and 2 patients had histologically normal squamous epithelium (follow-up, 33 months). 3 patients developed well differentiated verrucous SCC.

Conclusions: Esophageal SCC and lateral spread of neoplasia were diagnosed concomitantly in 41 patients $(42.7 \%) .18$ patients $(72 \%)$ with an initial biopsy diagnosis of lateral spread of neoplasia were later shown to have invasive carcinoma. Due to the high risk of subsequent detection of SCC in patients with lateral spread of squamous neoplasia in this study, we conclude that lateral spread of neoplasia is a marker of 
unsampled invasive SCC and may explain field carcinogenesis of squamous cell neoplasia. A microscopic diagnosis of lateral spread should therefore lead to careful mapping, as it could indicate an adjacent invasive SCC.

$680 \quad$ Upper Gastrointestinal Lesions in Peutz-Jeghers Syndrome Ogechukwu Pearl Eze, Yaman Tarabishy, Kevin Waters, Maryam Pezhouh, Francis Giardiello, Lysandra Voltaggio, Elizabeth A Montgomery. Johns Hopkins Hospital, Baltimore, MD.

Background: Peutz-Jeghers syndrome (PJS) is a hereditary autosomal dominant disorder characterized by multiple gastrointestinal (GI) hamartomatous polyps with a predilection for the jejunum, and mucocutaneous pigmentation. Affected patients are at increased risk of stomach, colorectal and pancreas adenocarcinoma. Since patients often present with intussusception and have small bowel lesions surgically resected, upper endoscopic findings for PJS are not well studied.

Design: Patients with PJS (7) who had undergone upper endoscopic biopsies that included gastric samples and whose slides were available for review were identified at a single institution over a 25 -year period. Upper endoscopies and corresponding upper GI biopsy specimens from these patients were reviewed. Biopsy specimens were further studied to characterize upper gastrointestinal lesions in Peutz-Jeghers patients. Results: The 7 patients consisted of 4 males and 3 females ranging in age from 1267 years. There were 18 gastric, 10 duodenal, and 2 jejunal polyps sampled from 14 endoscopic procedures in the 7 patients. Of a total of 18 gastric polyps, 3 could be diagnosed as PJ polyps based on their morphologic features (arborizing smooth muscle separating lobules of site specific mucosa), whereas the remaining 15 were indistinguishable from gastric hyperplastic polyps and characterized by foveolar hyperplasia with scant disorganized smooth muscle strands. One patient had a fundic gland polyp. One of the gastric polyps had low grade dysplasia (LGD). The background gastric mucosa, evaluable in 14 cases, lacked gastritis. There were 10 duodenal polyps sampled from the same patients all of which were non-diagnostic as PJ polyps. Two had LGD. There were 2 jejunal PJ polyps from a single patient, both without dysplasia. Conclusions: Patients with PJS who undergo upper tract screening frequently harbor gastric polyps, which are often non-diagnostic as PJ polyps in isolation without knowledge of the history of PJS. Since some have dysplasia, gastric screening should be included in the follow-up of patients with the syndrome.

681 Epstein-Barr Virus and Its Association with Fascin Expression in Colorectal Cancers in Syrian Population: A Tissue Microarray Study

Hanan Farghaly, Noor Al-Antary, Ala-Eddin Al-Moustafa. Hamad Medical Corporation (HMC), Doha, Qatar; Qatar College of Medicine, Doha, Qatar.

Background: Colorectal cancer (CRC) is the third most common malignancy in both men and women worldwide. Colorectal carcinogenesis is a complex, multistep process involving environmental and lifestyle features as well as sequential genetic changes. Epstein-Barr virus (EBV) has been recently shown to be present in human cancers, which could play an important role in the initiation and progression of these cancers. Fascin is an actin cross-linking protein involved in cell migration and adhesion. Little is known of EBV and its association with Fascin expression and colon cancer. This study explores the prevalence of EBV in CRC cases collected from Syrian patients using polymerase chain reaction (PCR) and tissue microarray (TMA) analysis.

Design: All diagnoses of CRC between 2005 and 2010 were identified. Diagnosis of colonic adenocarcinoma and grades were confirmed. A total of 102 colonic adenocarcinoma were identified ( 53 females and 49 males) with a median age of 49 years. Formalin fixed paraffin embedded tumor tissue blocks were selected for analysis. Five $\mu \mathrm{g}$ of purified DNA from each sample was analyzed for EBV by PCR using specific primers for LMP1 and EBNA1 genes. DNAs from human normal colorectal cells were used as negative controls for our PCR analysis. Immunohistochemical staining analysis of CRC was used for LMP1 and Fascin expression.

Results: Our results show that EBV is present in 37 out of 102 CRC patients (36.27\%). Additionally, the expression of LMP1 onco-protein of EBV was found to be directly correlated with Fascin expression/over-expression and tumor grade. The majority of CRC patients tumor with Fascin expression/over-expression are intermediate/high grade. This is the first report regarding the presence of EBV and its association with cancer phenotype and Fascin expression in CRC in the Middle East.

Conclusions: Our study supports the association of EBV and CRC and its prognostic significance of tumor grade. Consequently, additional studies are needed to expose the role of EBV in CRC initiation and progression.

682 Immunohistochemical Testing of Biopsy Specimens Is Warranted in Lynch Syndrome Screening

Jennifer J Findeis-Hosey, Karina Hiroshige, Bibiana Steinbauer. Univ. of Rochester Med. Ctr., Rochester, NY; University of Florida COM, Jacksonville, FL.

Background: Lynch syndrome (LS) most commonly results from germline mutations in the mismatch repair (MMR) genes $M L H 1, M S H 2, M S H 6$, and PMS2. Immunohistochemical (IHC) testing for the protein products of these genes can be performed on formalin-fixed paraffin-embedded tissue specimens as a screening tool for LS. We have previously reported a retrospective analysis of paired biopsy-resection colorectal carcinoma (CRC) specimens demonstrating 93\% concordance between biopsy and resection MMR protein status. Here we outline the results of our clinically-based prospective LS IHC screening in CRC biopsy specimens.

Design: All CRC specimens from Sept 2015-Sept 2016 were prospectively examined for MLH1, MSH2, MSH6, and PMS2 IHC staining. Nuclear staining in greater than $1 \%$ of tumor cells was considered positive. Forty-one patients were identified where IHC testing was performed at our institution on both the biopsy and resection specimens. IHC staining of the biopsy and resection specimens were examined.
Results: Of the 41 cases, the median age of diagnosis was 63 years old (range: $37-93$ years). Thirty-one $(75.6 \%)$ of the 41 cases demonstrated positive staining in all four MMR proteins on both biopsy and resection specimens. Ten (24.4\%) cases demonstrated loss of at least one MMR protein on resection specimen; nine of these cases demonstrated loss of the same MMR protein(s) on biopsy specimen. The one case where there was differing staining between the biopsy and resection specimens was a case of loss of MSH6 staining secondary to neoadjuvant treatment. This type of treatment-induced loss has been well documented and was not considered a discordant result. As such, the overall concordance rate was $100 \%$.

Conclusions: As screening of CRCs for LS markers becomes more commonplace, pathologists need to consider which specimens should be tested for greatest clinical impact. This data provides further evidence that MMR IHC testing of endoscopic biopsy specimens yields similar results to those obtained on resection specimens. While issues such as tumor heterogeneity have been raised previously, our data has not demonstrated any significant difference between the results found on biopsy and resection specimens.

\section{Crospovidone: A Common Incidental Finding in Gastrointestinal} Pathology Specimens

Jennifer J Findeis-Hosey, Raul S Gonzalez. Univ. of Rochester Med. Ctr., Rochester, NY. Background: Crospovidone is an inert polymer used as a filler substance in pharmaceutical tablets. The material is classified as a polyvinylpyrrolidone and has been studied in the lungs of intravenous drug users, but it has not been scrutinized in the gastrointestinal tract, to which it has much easier access.

Design: We identified fragments of crospovidone in 34 gastrointestinal pathology specimens from 33 patients; 30 of these were identified during a focused, six-month prospective analysis of incoming cases. Patient age and sex, organ, pathologic diagnosis, amount of crospovidone, and presence of other foreign material were noted.

Results: Median patient age was 65, and male:female ratio was 20:13. Two patients were children, one of whom had Crohn disease. The specimens included 31 biopsies and three resections, from the colon $(n=20)$, esophagus $(n=6)$, stomach $(n=4)$, small intestine $(n=2)$, appendix $(n=1)$, and gallbladder $(n=1)$. Crospovidone generally appeared as a coral-like substance colored a deep or shiny purple (Fig. 1A); it was pink in two cases. Material was scant in most specimens, though prominent in six. It was typically seen along the surface of the specimen, not invading or injuring tissue, though it was embedded within an ulcer in two cases; foreign body giant cell reaction was not seen, nor were foamy histiocytes characteristic of so-called "polyvinylpyrrolidone granuloma." Additional fecal debris was seen in 12 cases. The patient with crospovidone in the gallbladder had previously undergone biliary stenting (Fig. 1B). There was no particular association with clinical symptoms or with other pathologic diagnoses, which included colonic adenoma $(n=11)$, colonic adenocarcinoma $(n=2)$, sloughing esophagitis $(n=1)$, and iron pill gastritis $(\mathrm{n}=1)$.

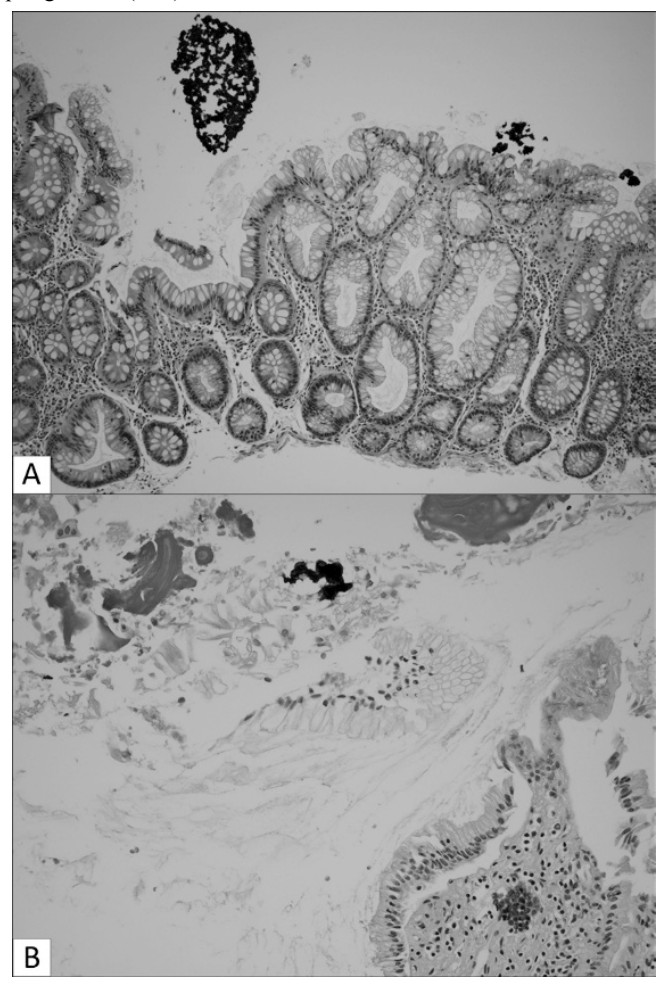

Conclusions: Crospovidone is an easily overlooked incidental finding in gastrointestinal specimens that appears to be somewhat common when sought. It causes no tissue damage or foreign body reaction, and it has no particular link to other pathologic diagnoses, including pill-induced injury. Its presence is of little consequence, though it should not be mistaken for calcifications (which may signify a pathologic process elsewhere) or other pill material (some of which is capable of causing tissue injury). 
$684 \quad$ High Level Microsatellite Instability in Appendiceal Carcinomas Wai Chin Foo, Michael Overman, Eduardo Vilar Sanchez, Susan Abraham, Melissa Taggart. M.D. Anderson Cancer Center, Houston, TX.

Background: High level microsatellite instability (MSI-H) is found in approximately $15 \%$ of all colorectal carcinomas (CRCs) and in at least $20 \%$ of right-sided colon cancers. In contrast, MSI-H appendiceal carcinomas are rare, with only 8 previously reported cases.

Design: We studied 176 appendiceal carcinomas in which MSI status was determined (using immunohistochemistry [IHC] for the DNA mismatch repair proteins MLH1, PMS2, MSH2, and MSH6 in 152 cases; by polymerase chain reaction [PCR] in 8 cases; and both IHC and PCR in 16 cases). Medical records were reviewed to determine personal and family histories of Lynch syndrome-type tumors; results of germline testing; and histologic features of the tumors in patients with MSI-H appendiceal carcinomas.

Results: Six (3.4\%) patients had MSI-H appendiceal carcinomas. These included 4 $(67 \%)$ females and $2(33 \%)$ males with a mean age of $45.8 \mathrm{yrs}$ (range, $32-59 \mathrm{yrs)}$. On histologic review, 5 tumors were comprised of invasive colonic-type adenocarcinomas ( 3 moderately differentiated, 2 poorly differentiated, 2 mucinous, and one with $<50 \%$ mucinous component) and one tumor was comprised of poorly differentiated adenocarcinoma with a variegated morphology including signet ring cell, mucinous, and undifferentiated components. In 2 patients, there was a known history of Lynch syndrome with deleterious MLH1 germline mutations and loss of MLH1/PMS2 staining in the appendiceal carcinomas by IHC. In 3 other cases, tumors showed loss of MSH2/ MSH6 expression; one of these patients underwent germline testing with no detected mutations in MSH2, MSH6, or EPCAM; the second carried a clinical diagnosis of Lynch syndrome and a significant family history of Lynch syndrome-associated carcinomas, but germline status was unknown; while the third had no known clinical or germline testing for Lynch syndrome. The final MSI-H appendiceal carcinoma demonstrated allelic shifts in 5 of 5 mononuclear repeats by PCR but intact IHC.

Conclusions: We report the largest series of appendiceal carcinomas tested for MSI status. Our findings confirm a previous study showing MSI-H in approximately $3 \%$ of appendiceal carcinomas. Unlike CRC, sporadic MSI-H due to MLH1 hypermethylation does not appear to occur in the appendix. The prevalence of MSI-H in appendiceal carcinoma is similar to the reported prevalence of MSI-H CRCs that are not associated with somatic hypermethylation of the MLH1 promotor. This suggests that MSI testing of appendiceal carcinomas has a similar efficacy to MSI testing of CRC for the detection of Lynch syndrome.

685 ALDH Expression in Serrated Polyps Highlights the Role of Stem Cells

Danielle Fortuna, Bruce Boman, Juan P Palazzo. Thomas Jefferson University Hospital, Philadelphia, PA.

Background: The study of stem cells (SCs) continues to evolve, as the cancer SC concept proves to play a significant role in understanding oncogenesis. In colon, discovering markers of these pluripotent cell compartments is actively investigated with aims to identify and potentially eliminate populations of tumor-initiating cells. Aldehyde dehydrogenase (ALDH), a ubiquitous subcellular oxidizing enzyme, modulates cell differentiation and proliferation and has been shown to be a marker of normal SCs residing in colonic crypts, as well as malignant human colonic SCs. As serrated lesion progression differs from the conventional carcinoma sequence, we examined ALDH expression by immunohistochemistry and the distribution of SCs in SPs (hyperplastic polyps (HP) and sessile serrated adenomas (SSA) \pm dysplasia).

Design: Immunohistochemistry was performed on 39 paraffin-embedded SPs $(6 \mathrm{HPs}, 30$ SSAs, 3 SSAs with dysplasia) and sections of normal colon using anti-ALDH antibody (BD Biosciences, San Jose, CA). The positive control was liver tissue. Staining intensity and distribution in both SP and available adjacent normal colonic mucosa was assessed. This study is IRB-approved.

Results: The estimated number of (individual strongly-positive) ALDH-SCs per crypt along with any cytoplasmic staining in the colonic epithelium was assessed. Multiple sections of normal colonic mucosa showed the following ALDH expression: approximately $20 \mathrm{ALDH}-\mathrm{SCs}$ per crypt (mostly in the SC niche) with weak cytoplasmic staining of the epithelium. $100 \%$ of SPs showed an overall decreased presence of ALDHSCs. $87 \%$ of adjacent normal colon also showed decreased ALDH-SCs. However, the majority of SPs $(74 \%)$ and adjacent normal mucosa $(67 \%)$ showed focal areas of abnormal, increased cytoplasmic staining of ALDH. Additionally, well-demarcated areas of increased ALDH staining highlighted presence of dysplasia in SSAs.

Conclusions: As a proxy of enzyme activity, ALDH staining highlights aberrant ALDH expression in SPs. Although the number of ALDH-SCs is decreased, SPs along with their adjacent normal mucosa show areas of overall increased ALDH cytoplasmic expression in the epithelium. Given that ALDH is a marker of "stem-ness," this may suggest that the serrated pathway involves preservation of SC-like metabolic properties, changes which also involve adjacent, histologically normal mucosa. These findings may help further explain the characteristics and continuum of serrated lesions and their progression.

686 Microscopic Tumor Deposits in the Liver Are Frequently Present in Patients with Liver Metastases from Digestive Neuroendocrine Tumors William Gibson, Raul S Gonzalez, Eric Liu, Chanjuan Shi. Vanderbilt University Medical Center, Nashville, TN.

Background: While most patients with digestive well-differentiated neuroendocrine tumor (NET) have an indolent clinical course, metastasis to the liver is frequent. Debulking surgery is commonly used to reduce tumor volume and eliminate tumorassociated syndromes, such as carcinoid syndrome. Here we present a series of digestive NET cases with liver metastases and examine the liver resections for microscopic tumor deposits.
Design: The Surgical Pathology archives were searched for digestive NETs with resected liver metastases. 117 cases were identified over a 14 year period (7/2002 to 8/2016). Among them, 73 cases had slides available for review and sufficient background liver for evaluation of microscopic tumor deposits. Microscopic tumor deposits were defined as grossly invisible, small tumor clusters $\leq 1 \mathrm{~mm}$ in size. Electronic medical records were also reviewed for patient demographics and follow-up data, including disease-specific survival.

Results: The set of 73 cases included 44 females and 29 males, with age ranging from 19 to 82 (mean age $=57$ ). It consisted of 45 small intestinal, 24 pancreatic, 2 duodenal, 1 ampullary, and 1 rectal NETs. Of the 73 total cases, $37(51 \%)$ had microscopic tumor deposits in the liver and $36(49 \%)$ did not. Of the two most common primary sites, the incidence of liver microscopic tumor deposits tended to be higher from the small intestine than from the pancreas $(28 / 45,62 \%$ versus $9 / 24,38 \% ; \mathrm{p}=0.076)$. The tumor deposits were seen in the portal tract with extension to the periportal region or in the lobules. Of the patients with tumor deposits, 15 died of the disease (41\%). Of the patients without tumor deposits, 3 died of the disease (8\%). Kaplan-Meier survival analysis after resection of liver lesions shows a 5 -year survival of $68 \%$ in those with tumor deposits compared to $97 \%$ for those without tumor deposits.

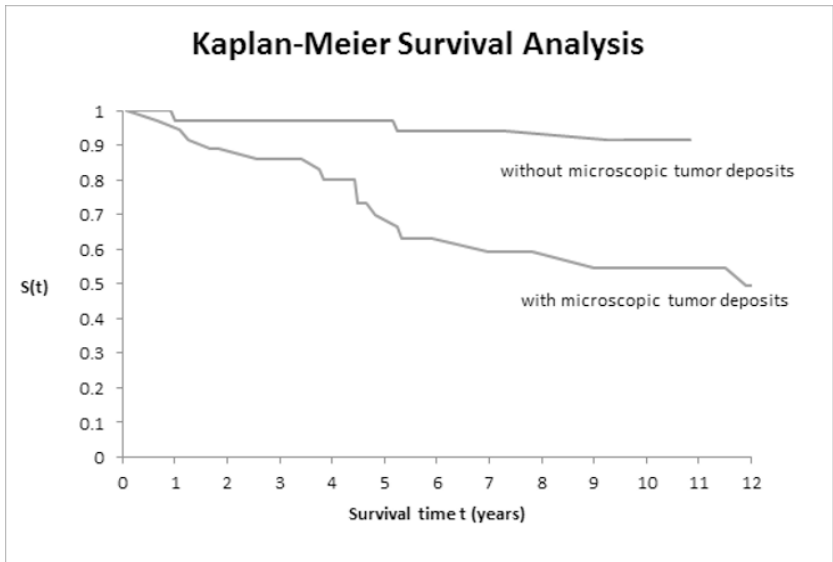

Conclusions: Microscopic tumor deposits in the liver are common in patients with metastatic digestive NETs and are associated with poor prognosis. These data suggest complete surgical resection of liver metastases is likely impossible in most of the patients. Other approaches such as systemic molecular targeted therapy (e.g. somatostatin-receptor based therapy) may be a better option.

687 Raman Spectroscopic Characterization of Submucosal Fat Deposition: A Marker of Inflammatory Bowel Disease?

William Gibson, Isaac J Pence, Molly Cone, Kay Washington, Anita MahadevanJansen. Vanderbilt University Medical Center, Nashville, TN; Vanderbilt University, Nashville, TN.

Background: Increased submucosal fat deposition of the bowel wall has been suggested in the radiology literature as a chronic feature of inflammatory bowel disease (IBD). Raman spectroscopy is an optical technique that probes the vibrational energy transitions of molecules during interaction with light. Here, we demonstrate ex vivo Raman spectra collected from eight partial colectomy specimens from IBD patients and correlate the signal data with histologic changes.

Design: Eight patients from a single institution who had a portion of their colon and/ or terminal ileum surgically removed from 5/2015 to 6/2016 were enrolled. Surgical indications were Crohn's disease (4), ulcerative colitis (3), and diverticulitis (1). A portable Raman spectroscopy system with $785 \mathrm{~nm}$ diode laser was used to grossly map 370-spectra across all specimens. 114 corresponding tissue sections were scored by a single pathologist for relative thicknesses of mucosa, submucosa and muscularis propria, presence and severity of active inflammation, mucosal crypt distortion, mucosal basal plasmacytosis, lymphoid hyperplasia, submucosal fat deposition, submucosal fibrosis, and submucosal vascular prominence. Histologic findings were compared to the Raman signals.

Results: Of the features examined, submucosal fat deposition showed the strongest correlation between Raman spectroscopy and histologic examination $(r=0.53$, $p$ $<0.001$ ). One case showed grossly homogenous mucosa with two markedly different spectroscopic signals taken a few centimeters apart, the only histologic difference being levels of submucosal fat (Fig. 2). Submucosal fat deposition showed strong correlation with IBD activity $(r=0.58, p<0.001)$ and mucosal crypt distortion $(r=0.58, p<0.001)$. The diverticulitis resection showed minimal active or chronic inflammatory changes and served as a control for the Raman spectroscopy signal. 


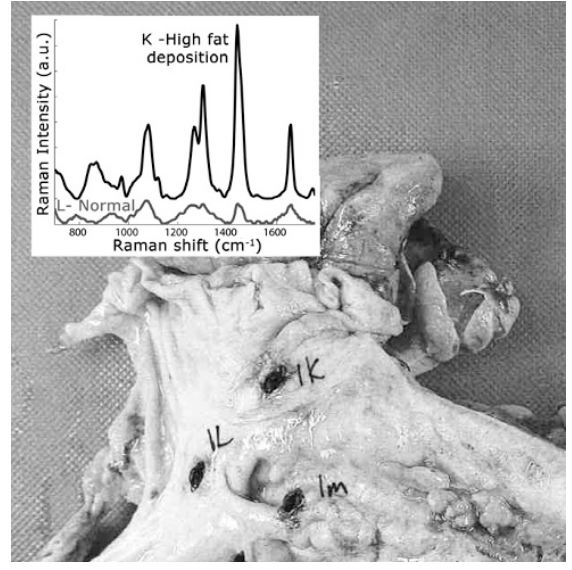

Conclusions: These preliminary results support the potential value of non-invasive assessment of submucosal fat deposition as a feature of IBD. Raman spectroscopy is a potential tool to further study this relationship. In the future, endoscopic application of Raman spectroscopy may serve as a diagnostic adjunct to tissue biopsy.

688 Detection of Mutations in DNA Polymerase $\varepsilon$ (POLE) in Colorectal Carcinoma with Intact Mismatch Repair Proteins: Immunotherapeutic Implications of Ultramutation

Adam J Gomez, Ann Burton, David Steiner, James Zehnder, Teri Longacre. Stanford Health Care, Stanford, CA.

Background: Analysis of The Cancer Genome Atlas data identified fifteen colorectal carcinomas (CRC) that had one or more mutations in DNA polymerase $\varepsilon$ (POLE) and demonstrated hypermutation phenotypes similar to carcinomas with microsatellite instability (MSI), although half of these POLE-mutated cases were either microsatellite stable or had low-level MSI. There is little known about the morphologic spectrum and response to therapy in CRC with POLE mutations, although these tumors have demonstrated an increase in neoantigens and enrichment for the presence of inhibitory molecules including PD-L1.

Design: A retrospective search of our pathology database was performed to identify consecutive CRC resection specimens with intact mismatch repair protein (MMR) expression by immunohistochemistry from January 2015 to April 2016 . We performed PCR using primers targeting the POLE mutation hotspots in the exonuclease domain with subsequent Sanger sequencing. Additional cases were identified by our institution's next-generation sequencing (NGS) somatic mutation panel that includes POLE. Cases were reviewed to assess tumor morphology and cellularity.

Results: Of 64 consecutive CRC resection specimens with intact MMR, 4 carcinomas from 3 patients had POLE exonuclease domain mutations (Table 1). Additionally, 1 metastatic CRC with intact MMR was found to harbor a POLE mutation by NGS performed on the lymph node specimen (patient 2).

\begin{tabular}{|c|c|c|c|}
\hline Patient & POLE Mutation & Morphology & Tumor $\%$ \\
\hline 1 & c. $857 \mathrm{C}>\mathrm{G}$, p.P286R & Increased TIL & $90 \%$ \\
\hline 2 & c. $1231 \mathrm{G}>\mathrm{T}, \mathrm{p} . \mathrm{V} 411 \mathrm{~L}$ & Mucinous/signet ring & $<10 \%$ \\
\hline $\begin{array}{c}3(2 \\
\text { foci })\end{array}$ & $\begin{array}{l}\text { c. } 1092 \mathrm{G}>\mathrm{A}, \mathrm{p} \cdot \mathrm{G} 364 \mathrm{Gc} \cdot 1092 \mathrm{G}>\mathrm{A}, \\
\text { p.G364G }\end{array}$ & $\begin{array}{l}\text { Mucinous/signet } \\
\text { ringMicropapillary }\end{array}$ & $80 \% 80 \%$ \\
\hline 4 & c. $857 \mathrm{C}>\mathrm{G}, \mathrm{p} . \mathrm{P} 286 \mathrm{R}$ & Increased TIL & $90 \%$ \\
\hline
\end{tabular}

Conclusions: In this study, we demonstrate 3/64 (4.7\%) of consecutive MMR-intact $\mathrm{CRC}$ resection specimens and 1 metastatic CRC with intact MMR harbor POLE mutations with histologic features of increased TIL \& mucinous/signet ring morphology. Given the ultramutation phenotype and increased neoantigen formation in $P O L E$ mutated CRC, 2 patients were considered for treatment as MMR-deficient carcinomas with pembrolizumab targeting the PD-1 receptor. Ultimately, clinical follow-up of these patients will further our understanding of the biological response to novel therapies targeting PD-1 and PD-L1 in the setting of concurrent POLE mutation and intact MMR.

\section{Gastroblastoma Harbors Recurrent Somatic MALAT1-GLI1}

\section{Fusion Gene}

Rondell Graham, Asha Nair, Jaime Davila, Long Jin, Patrick P Bedroske, Jin Jen, William R Sukov, Tsung-Teh Wu, Henry D Appelman, Jorge Torres-Mora, Kyle D Perry, Lizhi Zhang, Andrew L Folpe. Mayo Clinic, Rochester, MN; University of Michigan, Ann Arbor, MI.

Background: Gastroblastoma is a rare distinctive biphasic tumor of the stomach. The molecular biology of gastroblastoma has not been studied.

Design: We retrieved 2 gastroblastomas from the consultation practices of the authors and performed transcriptome sequencing on formalin fixed paraffin embedded tissue. The data were analyzed with the MAP-RSeq bioinformatics pipeline. Recurrent predicted fusion genes were validated at genomic and RNA levels by FISH and RT-PCR. The presence of the fusion gene was confirmed on an additional paraffin embedded case of gastroblastoma. Control cases of synovial sarcoma $(n=7)$, GIST $(n=2)$, plexiform fibromyxoma $(n=2)$, leiomyoma $(n=5)$, leiomyosarcoma $(n=5)$, Ewing sarcoma $(n=5)$, Wilms tumor $(\mathrm{n}=2)$, desmoid-type fibromatosis $(\mathrm{n}=5)$ and normal gastric mucosa and muscularis propria $(\mathrm{n}=2)$ were also analyzed by FISH and RT-PCR.
Results: The gastroblastomas affected 2 males ( 9 and 30 years old) and a 25 -year-old woman. The tumors arose from the gastric antrum $(\mathrm{n}=2)$ and body. In the first case, a lymph node metastasis was also present. Both initial gastroblastomas harbored recurrent somatic MALAT1-GLII fusion transcripts which were predicted to retain the key domains of GLI1. The fusion gene was validated at the genomic levels by break apart and dual fusion FISH and at RNA levels. The metastasis and the $3^{\text {rd }}$ case of gastroblastoma also harbored the MALAT1-GLII fusion transcript. A reciprocal fusion transcript was identified in all 3 cases. As expected, plexiform fibromyxoma was positive for the MALAT1-GLI1 gene but none of the other control tissues harbored MALAT1-GLI1. qPCR and immunohistochemistry confirmed overexpression of GLI1 in the cases of gastroblastomas.

Conclusions: We have identified an oncogenic MALAT1-GLII gene in 3 cases of gastroblastoma. The fusion gene is predicted to encode a protein which includes the zinc finger domains of GLI1 and results in overexpression of GLI1 protein. Ongoing gene expression profiling and pathway analysis may help to elucidate the relationship between gastroblastoma and plexiform fibromyxoma.

690 Histopathology of Chronic Antibody-Mediated Rejection in Pediatric Liver Transplantation: Is Obliterative Portal Venopathy a Novel Finding?

Marjorie-Anne R Guerra, Laura J Wozniak, Jason V Scapa, Bita V Naini. UCLA, Los Angeles, CA.

Background: Obliterative portal venopathy (OPV) is a cause of non-cirrhotic portal hypertension and is characterized by luminal narrowing or loss of small portal veins with or without nodular regenerative hyperplasia. OPV has previously been reported as a histopathologic finding in adult liver transplant recipients who have circulating donor-specific antibodies (DSA). The Banff Working Group on Liver Allograft Pathology recently published diagnostic criteria for chronic antibody-mediated rejection (AMR) in liver allografts, and defines characteristic histopathologic findings in the presence of circulating HLA DSAs within three months of biopsy. However, the specified histopathologic findings do not include OPV. Our aim is to describe OPV in association with other histopathologic features in pediatric liver transplant recipients with chronic AMR.

Design: We evaluated liver biopsies from pediatric liver transplant recipients over a two year period of 2014-2016, who had OPV on liver biopsy and were clinically diagnosed with chronic AMR. Four patients were identified and a complete histopathologic evaluation was performed in association with liver biochemistry and presence of DSAs. Results: The four patients ranged in age from 6 to 18 years of age and received their liver transplant from 2 to 14 years prior. All four patients had OPV and circulating class II DSAs. All the cases had at least minimal portal and/or perivenular inflammation and mild to moderate portal or sinusoidal fibrosis, which are features consistent with chronic AMR. Although none of the cases showed cirrhosis, three cases had associated nodular regenerative hyperplasia present. Portal vein thrombosis and/or stenosis was excluded in all cases by MR venogram. Interestingly, all four patients had varying clinical features of portal hypertension. While only one case had C4d stain performed, it was found to be diffusely positive. Two patients ultimately experienced graft failure and are currently re-listed for transplant.

Conclusions: OPV is a histopathologic finding associated with chronic AMR in pediatric recipients of liver allografts. Clinicians caring for liver transplant recipients with OPV should consider checking for the presence of DSA, particular class II, to evaluate underlying etiology of portal venopathy in order to rule out AMR. Further studies are needed to elucidate whether portal venopathy is amenable to medical therapy.

691 Challenges in Equivocal Score (2+) HER2 Results for Gastric/ Gastroesophageal Junction Adenocarcinomas

Shaheed W Hakim, Hala Faragalla, Kiran Jakate, Catherine Streutker. St. Michael's Hospital, Toronto, ON, Canada.

Background: HER2 is expressed in $10-25 \%$ of gastric/gastroesophageal adenocarcinomas (GA) and is targetable with trastuzumab. Patients with HER $23+$ by immunohistochemistry (IHC) or 2+/equivocal staining with amplification by in situ hybridization (ISH) are eligible for therapy. Variance in interpretation of what constitutes $2+/$ equivocal staining can lead to increased numbers of cases tested by ISH, which will increase costs and potentially delay treatment. We explore the variables that may hinder a more definitive score on IHC.

Design: 54 patients with GA and with HER2 2+ IHC staining and their corresponding ISH results were obtained from our database. Cases were assessed for histological subtype of GA, HER2 staining intensity and staining pattern. Immunoreactivity for $2+/$ equivocal staining was assessed by the strict definition used in the ToGA trial criteria - tumour clusters $(<$ or $=5$ cells) with weak to moderate complete, basolateral or lateral membranous reactivity, visible at 10-20x magnification. ISH results were then compared with each variable.

Results: Of the 54 cases, $74 \%$ (40/54) were assessed to have a true score of $2+.22 \%$ $(12 / 54)$ were rescored as $1+$ and $4 \%(2 / 54)$ were rescored as $3+$. ISH showed HER2 amplification $(\geq 2.0)$ in $15 \%(8 / 54)$. None of the cases rescored as $1+$ on IHC were amplified by ISH. Both cases rescored as 3+ were amplified on ISH. Cytoplasmic HER2 staining was observed in 14 of the total cases. Of the cases rescored as $1+, 3$ were diffuse gastric cancer; 2 of these had significant cytoplasmic staining. 2 intestinal type adenocarcinomas also showed cytoplasmic staining; one of these was rescored as a 3+. 2 of the cases with significant cytoplasmic staining on HER2 IHC were ISH+.

Conclusions: Assessment for HER2 scoring can be subjective; benefit of the doubt is often given for borderline staining, resulting in increased rates of ISH. Our results show a significant rate of downgrading of cases from $2+$ to $1+$ staining, with occasional upgrading to $3+$. Our rate of IHC $2+/ \mathrm{ISH}+$ was $15 \%$ before rescoring and $20 \%$ after; this is lower than $27 \%$ seen in the ToGA trial, consistent with the inclusion of cases which are actually negative on IHC. These results suggest that applying strict criteria is 
more cost effective and does not miss cases which are truly positive. However, variables such as cytoplasmic staining can act as confounders. Ultimately, for the truly borderline and challenging scores, ISH should be considered for the advocacy of patient care.

\section{Novel Classification of Dysplasia in IBD}

Noam Harpaz, John R Goldblum, Neil Shepherd, Robert H Riddell, Carlos A Rubio, Michael Vieth, Robert D Odze. Icahn School of Medicine at Mount Sinai, New York, NY; Cleveland Clinic, Cleveland, OH; Gloucestershire Cellular Pathology Laboratory, Gloucester, United Kingdom; Mount Sinai Hospital, Toronto, Canada; Karolinska Institutet, Stockholm, Sweden; Institute of Pathology, Bayreuth Clinic, Bayreuth, Germany; Brigham and Women's Hospital, Boston, MA.

Background: The classification system for diagnosis and grading of dysplasia in IBD proposed in 1983 by Riddell et al. has been widely adopted as the standard for clinical and research purposes, but there has been little subsequent effort to address the recently recognized morphological and biological diversity of dysplasia. The aim of this study was to organize dysplasia into morphological categories so that future studies on their biology and natural history can be performed.

Design: Seven GI pathologists with particular research expertise in IBD contributed a total of 200 electronic images of dysplasia. Two pathologists (NH and RO) collated and reviewed all of the images and separated them into morphologic categories based on a variety of architectural and cytologic features. A test set of 35 images was sent to the other participants who were asked to place them into one of these categories if possible. Each category was represented by 1 or 2 sample images and a written description of its morphologic features.

Results: Seven categories of dysplasia were recognized. They included dysplasia with terminal epithelial differentiation (Type 1), sessile serrated polyp/adenoma-like dysplasia (Type 2), traditional serrated adenoma-like dysplasia (Type 3), conventional adenoma-like dysplasia (Type 4), hypermucinous dysplasia (Type 5), goblet cell depleted dysplasia (Type 6), and serrated dysplasia NOS (Type 7). Overall, diagnostic agreement for each dysplasia category was very good, with $\geq 4$ pathologists in agreement in $23 / 35$ cases $(66 \%)$. Diagnostic agreement was highest for Type 6 (92\% agreement), intermediate for Types $1,2,4,5$ and $7(\geq 60 \%$ agreement) and lowest for Types 3 $(<50 \%$ agreement) . Types 1 and 6 have not been formally described hitherto. They are characterized by flat configuration, non-crowded crypt pattern and cytoplasmic features that either simulate the repertoire of normal colonic epithelial cells (Type 1) or are devoid of goblet cells (Type 6)

Conclusions: We successfully classified the broad spectrum of morphologic dysplasia in IBD into 7 distinct categories. The system was validated and shown to result in very good agreement by expert GI pathologists. This classification should provide a basis for further studies of the biology and natural history of the various subtypes of dysplasia.

693

TFE3 and SOX11 as Novel Diagnostic Markers for Solid Pseudopapillary Neoplasms

Grant Harrison, Amanda Hemmerich, Diana Cardona, Cynthia Guy, Shannon McCall, Xuefeng Zhang. Duke University Medical Center, Durham, NC.

Background: Solid pseudopapillary neoplasms (SPN) are rare malignant neoplasms with an indolent disease course. The diagnosis of SPN can be straightforward in cases with typical morphology and immunohistochemical profiles; however, morphologic variants or small samples can make the histologic diagnosis more challenging. Other pancreatic tumors, including acinar cell carcinoma, neuroendocrine tumor, and pancreatoblastoma, may share similar features as SPN, potentially hindering a definitive diagnosis in selected cases. In this study, we propose transcription factor E3 (TFE3) and SOX11 as novel diagnostic markers for SPN. TFE3 is involved in the Wnt/ $\beta$ catenin signaling pathway, which is vital in the pathogenesis of SPN. Previously, we have shown TFE3 to be a sensitive and specific marker for SPN (16 cases). SOX11 is a transcription factor, and is upregulated in mantle cell lymphoma and glioma. SOX11 mRNA overexpression has been recently reported in SPN.

Design: The pathology databases were searched from 2000 to 2015 . Thirty-one cases of surgically resected SPN were retrieved. Other types of tumors from the same time period were used as controls, including consecutive cases of acinar cell carcinoma (2), pancreatoblastoma (3), and selected neuroendocrine tumors (24). Immunohistochemistry for TFE3 and SOX11 was performed and scored independently by 2 pathologists in a masked manner.

Results: Nuclear immunoreactivity for TFE3 was detected in 30/31 of the SPN and in $4 / 29$ of the control tumors, resulting in a sensitivity of $97 \%$ and specificity of $86 \%$. Nuclear immunoreactivity for SOX11 was detected in all of the SPN and in 5/29 of the control tumors, resulting in a sensitivity of $100 \%$ and specificity of $83 \%$. When the 2 markers were combined and tumors with immunoreactivity to both markers were considered as positive, the specificity was increased to $93 \%$, with a slight sacrifice of sensitivity to $97 \%$. The results were summarized in Table 1 .

\begin{tabular}{|l|c|c|c|c|}
\hline \multicolumn{5}{|l|}{ Table 1: Diagnostic values of TFE3 and SOX11 in SPN } \\
\hline & Sensitivity & Specificity & Positive predictive value & Negative predictive value \\
\hline TFE3 & $97 \%$ & $86 \%$ & $88 \%$ & $96 \%$ \\
\hline SOX11 & $100 \%$ & $83 \%$ & $86 \%$ & $100 \%$ \\
\hline Both + & $97 \%$ & $93 \%$ & $94 \%$ & $96 \%$ \\
\hline Either + & $100 \%$ & $76 \%$ & $82 \%$ & $100 \%$ \\
\hline
\end{tabular}

Conclusions: TFE3 and SOX11 are highly sensitive and specific immunohistochemical markers for SPN, and may be of value in distinguishing between SPN and other pancreatic tumors. The combination of the 2 markers offers optimized sensitivity and specificity.
$694 \quad$ Histology of Colorectal Adenocarcinoma with Double Somatic Mismatch Repair Mutations Is Indistinguishable from Those Caused by Lynch Syndrome

Jessica Hemminger, Rachel Pearlman, Sigurdis Haraldsdottir, Deborah Knight, Heather Hampel, Wendy L Frankel. The Ohio State University Wexner Medical Center, Comprehensive Cancer Center, Columbus, $\mathrm{OH}$.

Background: Lynch syndrome (LS) is an inherited cancer predisposition syndrome caused by germline mutations in mismatch repair (MMR) genes, $\mathrm{MLH1}, \mathrm{MSH} 2$ (EPCAM), MSH6, and PMS2, followed by a second hit to the remaining allele leading to cancer. Universal tumor screening among newly diagnosed colorectal carcinomas (CRC) for LS, using microsatellite instability (MSI) and/or immunohistochemistry (IHC), has identified tumors with unexplained MMR deficiency (abnormal screening, absent $\mathrm{MLH1}$ methylation, no detectable germline MMR mutation). It is known that $68 \%$ of patients with unexplained MMR deficiency have double somatic (DS) mutations in the MMR gene corresponding with the absent protein by IHC. We determined whether histomorphology could distinguish patients with DS mutations from those with LS.

Design: CRC patients with DS mutations were identified from population based cohorts of CRC from Iceland (2000-2009), Columbus, Ohio (1999-2005), and the state of Ohio (2013-present). Next-generation sequencing was performed using Coloseq Tumor (University of Washington) on available tumors with unexplained MMR deficiency. Patients with LS from Ohio cohorts were the comparison group. Age, gender, tumor site and histologic features were evaluated. Tumor infiltrating lymphocytes (TIL) and Crohn's-like reaction (CLR) were scored 0,1 , and 2. Histologic subtypes associated with MSI (mucinous, signet ring, poorly differentiated, medullary) and necrosis were noted. Results: We identified 43 tumors with DS mutations and 48 from patients with LS. Compared to LS, tumors with DS mutations were from older patients $(p=0.001)$ and involved right or transverse more often than left colon $(p=0.003)$. There was no significant difference in gender or histologic features. The majority of DS tumors had mutations in $M L H 1(56 \%)$ and $M S H 2(33 \%)$, while most LS tumors had mutations in MSH2 (50\%) and MLH1 (21\%).

\begin{tabular}{|c|c|c|c|c|c|c|c|}
\hline & $\begin{array}{c}\text { Age, mean } \\
\text { (SD) }\end{array}$ & $\mathrm{M} / \mathrm{F}$ & $\begin{array}{c}\text { Right or } \\
\text { Transverse/ } \\
\text { Left }\end{array}$ & $1+/ 2+$ & $1+/ 2+$ & Necrosis & $\begin{array}{c}\text { MSI } \\
\text { Histology }\end{array}$ \\
\hline $\begin{array}{c}\mathrm{DS} \\
(\mathrm{n}=43)\end{array}$ & $60.3(14.6)$ & $\begin{array}{c}60 \% / \\
40 \%\end{array}$ & $88 \% / 12 \%$ & $\begin{array}{c}26 \% / \\
56 \%\end{array}$ & $\begin{array}{c}37 \% / \\
44 \%\end{array}$ & $37 \%$ & $86 \%$ \\
\hline $\begin{array}{c}\mathrm{LS} \\
(\mathrm{n}=48)\end{array}$ & $51.3(10.9)$ & $\begin{array}{c}57 \% / \\
43 \%\end{array}$ & $61 \% / 39 \%$ & $\begin{array}{c}15 \% / \\
60 \%\end{array}$ & $\begin{array}{c}38 \% / \\
40 \%\end{array}$ & $36 \%$ & $73 \%$ \\
\hline
\end{tabular}

Conclusions: The histology of tumors with DS mutations is indistinguishable from those caused by LS. Tumor sequencing for evaluation of DS mutations is recommended for patients with unexplained MMR deficiency to clarify sporadic versus hereditary CRC.

\section{9} with Literature Review

Richard A Hickman, Azore-Dee Bradshaw, Abul Ala Syed Rifat Mannan, Antonio G Neto, Susan Kornacki, Cristina Hajdu, Neil Theise, Jonathan Melamed. NYU Langone Medical Center, New York, NY; Mount Sinai Hospital, New York, NY.

Background: Russell body gastritis (RBG) is a rare chronic inflammatory condition in which there is a localized mucosal accumulation of plasma cells filled with immunoglobulins. The clinicopathologic significance of RBG is poorly understood. We performed a multicenter, retrospective study of RBG and incorporated published reports to further clarify the significance of this lesion.

Design: We retrospectively identified cases of RBG using natural language searches for "Russell body" and "Russell body gastritis" at 3 tertiary care institutions since 1998 until September 2016. PubMed search terms included "Russell body gastritis". Cases were reviewed, tabulated and incorporated into this series.

Results: Within our institutions, 9 cases were reported with findings consistent with RBG; an additional 32 cases were found on PubMed. All cases were biopsy specimens. Indications for biopsy were mostly dyspepsia $(n=25,61 \%)$. Twenty-three $(55 \%)$ patients were men and the mean patient age was 62 years (range: $24-88$ years). The most commonly affected site was antral mucosa $(56 \%)$, followed by oxyntic mucosa $(29 \%)$, gastroesophageal junction $(11 \%)$ and the transition zone $(4.4 \%)$. H. pylori was associated in 18 cases $(43 \%)$, usually at the site of RBG $(n=16,89 \%)$. For those cases not associated with $H$. pylori, 3 were associated with immunodeficiency (HIV or receiving immunosuppressive therapy) and 1 at the site of iron pill gastritis.

Of 41 cases, 36 cases were assessed for kappa/ lambda light chain restriction (LCR), 7 of which exhibited LCR. Of cases with LCR, 1 had MGUS and another had MALT lymphoma. Three cases without documented LCR developed a subsequent lymphoproliferative disorder (LD).

\begin{tabular}{|l|l|l|l|l|l|}
\hline Cohorts & $\begin{array}{l}\text { H. pylori } \\
\text { associated }\end{array}$ & $\begin{array}{l}\text { LCR /cases } \\
\text { evaluated } \\
\text { for LCR }\end{array}$ & LD & Site & LD \\
\hline $\begin{array}{l}\text { Current study } \\
(\mathrm{n}=9)\end{array}$ & 1 of 9 & $2 / 5$ & $\begin{array}{l}1 \\
\text { of } 9\end{array}$ & $\begin{array}{l}\text { Extra-gastric } \\
(\mathrm{n}=1)\end{array}$ & $\begin{array}{l}\text { Mantle cell lymphoma } \\
(\mathrm{n}=1)\end{array}$ \\
\hline $\begin{array}{l}\text { Literature case } \\
\text { review (n=32) }\end{array}$ & 17 of 32 & $5 / 31$ & $\begin{array}{l}4 \text { of } \\
32\end{array}$ & $\begin{array}{l}\text { Extra-gastric } \\
(\mathrm{n}=3) \\
\text { Gastric }(\mathrm{n}=1)\end{array}$ & $\begin{array}{l}\text { MGUS (n=1) } \\
\text { Post-transplant LD } \\
(\mathrm{n}=1)\end{array}$ \\
$\begin{array}{l}\text { Multiple myeloma }(\mathrm{n}=1) \\
\text { Gastric MALT } \\
\text { lymphoma (n=1) }\end{array}$ \\
\hline Total (n=41) & 18 of 41 & $7 / 36$ & $\begin{array}{l}5 \text { of } \\
41\end{array}$ & & \\
\hline
\end{tabular}


Conclusions: RBG is a rarely reported entity, typically found incidentally within antral mucosa in association with $H$. pylori infection. A small proportion of cases are associated with a LD, often in extra-gastric locations. Cases of RBG should be considered for plasma cell LCR analysis. The association between RBG and LD warrants further evaluation to determine the risk, if any, for later LD.

696

Examination of Luminal Debris Aids Distinction Between Peptic Ulcers and Ulcerated Gastric Cancers

Erika Hissong, Jose Jessurun, Rhonda K Yantiss. New York Presbyterian-Weill Cornell Medicine, New York, NY.

Background: Ulcerated gastric carcinomas can be difficult to distinguish from peptic ulcers, especially in small biopsy samples. Cancers often show superficial necrosis colonized by bacteria and/or fungi due to underlying atrophic gastritis, hypochlorhydria, and incomplete sterilization of luminal contents. On the other hand, most peptic ulcers develop in patients with adequate acid production; organisms encountered in biopsy samples usually represent $H$. pylori or oral contaminants. We performed this study to determine whether analysis of luminal debris distinguishes benign gastric ulcers from ulcerated cancers.

Design: We retrospectively identified H\&E-stained sections from 100 gastric biopsy samples, including 50 ulcerated adenocarcinomas (study cases) and 50 benign ulcers (controls). Luminal debris was evaluated for type of inflammation, karyorrhectic debris, blood, fibrin, bacteria, and yeast; H. pylori infection, gastritis, chemical gastropathy, and intestinal metaplasia were noted when present in background mucosae. Confirmatory Warthin-Starry stains were performed in all cases.

Results: Study patients (mean: 69 years) and controls (mean: 65 years) were similar. Cancers occurred throughout the stomach and showed tubular $(n=23,46 \%)$, diffuse $(\mathrm{n}=15,30 \%)$, or mixed $(\mathrm{n}=11,22 \%)$ morphology; one case was a mucinous carcinoma. Study cases showed intestinal metaplasia more frequently than controls $(50 \% \mathrm{vs}$. $28 \%, \mathrm{p}=0.04)$ with less frequent chemical gastropathy $(10 \% v s .52 \%, \mathrm{p}=0.0002)$ in background mucosae. Neutrophils, eosinophils, karyorrhectic debris, fibrin, and blood were commonly present in luminal debris in both groups. However, most cancers contained numerous, readily apparent non-H.pylori bacteria $(n=38,76 \%)$, filamentous bacteria $(\mathrm{n}=8,16 \%)$, and/or fungi $(\mathrm{n}=9,18 \%)$. Only $5(10 \%)$ peptic ulcers contained non- $H$. pylori bacteria $(\mathrm{p}=0.0001)$, which were usually scant. Filamentous bacteria and yeast were identified in one control $(2 \%)$ with atrophic gastric mucosa $(\mathrm{p}=0.015$ and $\mathrm{p}=0.008$ compared to study group).

Conclusions: Gastric carcinomas often develop in patients with hypochlorhydria and are frequently colonized by bacteria and/or fungi. In contrast, non-H. pylori organisms are infrequently detected in exudates from benign ulcers. Detection of filamentous bacteria or fungi in luminal debris of gastric "ulcers" should raise suspicion for malignancy, prompting careful correlation with endoscopic findings, deeper tissue sections, or additional sampling.

697 Polypoid Hyperplasia of the Colonic Mucosa (PH). A Pathologic and Molecular Reassessment of Endoscopically but Not Histologically Apparent Polyps

Erika Hissong, Helen Fernandes, Jose Jessurun. New York Presbyterian-Weill Cornell Medicine, New York, NY.

Background: Endoscopically apparent colonic polyps are frequently biopsied which lack the microscopic features of well-characterized, non-dysplastic or dysplastic polyps. Diagnostic terms used for these lesions range include normal mucosa, benign mucosal polyp and goblet cell rich hyperplastic polyp. This study was designed to characterize these lesions.

Design: We prospectively collected 169 polyp biopsies that lacked the microscopic characteristics of conventional polyps. Polypoid mucosa was compared with normal on the same slide. Crypts were assessed for cellularity, cell type, length/thickness, luminal width, serration, and nuclear stratification, hyperchromasia and polarization. Next generation sequencing (NGS) was performed on 30 polyps using a targeted panel interrogating somatic single nucleotide variants in 50 cancer-related genes.

Results: There were 81 males and 88 females, with a mean age of 59 years. All lesions were sessile and less than $0.5 \mathrm{~cm}$. The polypoid mucosa was expanded by elongated and widened, non-serrated tubular crypts with distended luminal openings. Goblet cells were similar or slightly increased compared to the background mucosa. No nuclear abnormalities or increased mitoses were present. PH represented $11 \%$ of all polyps. Synchronous hyperplastic, sessile serrated adenomas and dysplastic polyps were found in $30 \%, 7 \%$, and $51 \%$ of patients respectively. Most polyps were located in the distal colon and rectum $(60 \%)$ followed by the proximal colon $(29 \%)$ and transverse colon $(11 \%)$. Molecular analysis of 30 cases showed $K R A S$ mutations in $31 \%$ of polyps and $B R A F$ V600E mutation in one polyp. These mutations were not found in 20 samples of normal colonic mucosa.

Conclusions: Polyps which lack the typical features of conventional serrated or dysplastic polyps show subtle but identifiable changes of mucosal hyperplasia. Moreover, $31 \%$ of these polyps show KRAS mutations. We propose the term polypoid hyperplasia of the colonic mucosa rather than goblet cell rich hyperplastic polyp or other terms to conceptually separate these polyps from the serrated polyp family since there are differences in their histologic and molecular characteristics. Their clinical significance, if any, awaits to be determined.

\section{Appendicitis}

Bela Horvath, Ian Brown, Namrata Setia, Anthony Mattia, Laura Lamps, Gregory E Lauwers, Joseph Misdraji. Massachusetts General Hospital, Boston, MA; Envoi Specialist Pathologists, Kelvin Grove, QLD, Australia; University of Chicago, Chicago, IL; University of Arkansas for Medical Sciences, Little Rock, AR; University of California, San Francisco, CA.

Background: Actinomycosis is a relatively rare chronic infection caused by filamentous, Gram-positive bacteria. It most commonly involves the cervicofacial region, but a few case reports describe Actinomyces as a causative agent in appendicitis. We and others have encountered rare cases of appendices with Crohn's-like features that had Actinomyces. We aimed to characterize the histologic features of this rare entity and to determine if Crohn's-like histology was characteristic of Actinomyces-related appendicitis.

Design: Cases were contributed from 5 institutions, either from consults or cases identified during routine sign-out. Additionally, the surgical pathology files at MGH were searched over a 5 years period to find cases, by searching for the term Actinomyces but also Crohn's, transmural, granuloma, or chronic appendicitis: 34 cases were reviewed and 3 cases with Actinomyces and typical histology were included. A control group consisted of 100 consecutive cases of acute appendicitis

Results: We collected 19 appendectomy specimens with Actinomyces. Four cases presented as a mass lesion with clinical concern for malignancy. On histologic review, 14 cases had characteristic morphology, with moderate to marked lymphoid hyperplasia, transmural lymphoid aggregates, and marked periappendiceal fibrosis. Six cases also had granulomas, four with numerous granulomas. In the control group, one specimen had Actinomyces in the lumen but did not show typical morphology. None of the other controls had a Crohn's-like appearance. Of 8 patients with follow up, one (with numerous granulomas) developed symptoms compatible with Crohn's disease.

Conclusions: Actinomyces can cause a Crohn's-like appearance, with follicular hyperplasia, transmural inflammation, and occasionally granulomas. Recognition of this pattern could help pathologists to consider Actinomycotic appendicitis when an appendix is submitted with a clinical concern for malignancy and/or has histologic features that suggest Crohn's disease or "chronic appendicitis".

699 Clinicopathologic Features of Low-Grade Appendicea Mucinous Neoplasms: A Single-Institution Experience of 104 Cases

Aaron R Huber, Jennifer J Findeis-Hosey, Raul S Gonzalez. University of Rochester Medical Center, Rochester, NY.

Background: Low-grade Appendiceal Mucinous Neoplasm (LAMN) is an enigmatic tumor that replaces normal epithelium with low-grade mucinous epithelium. They lack the capacity for destructive invasion but can dissect through the appendiceal wall, extruding mucin and/or epithelium and causing pseudomyxoma peritonei (PP). This behavior is well-known, but the histologic spectrum of LAMN has not been fully examined.

Design: We identified 104 cases of previously diagnosed LAMN or "mucinous cystadenoma." H\&E-stained slides from each were reviewed, and pathologic parameters were noted, including diagnosis, size, whether the appendix was entirely submitted or dilated, wall involvement, margin status, grade of cytologic atypia, and presence of mucosal Schwann cell proliferation and/or PP. Clinical parameters noted included age, race, sex, radiographic findings, and outcome.

Results: The patients were 64 females and 40 males, with a median age of 59 years (range: 15-95). Most $(90,87 \%)$ were white. History was available for 97 patients. The majority $(60,62 \%)$ were symptomatic, typically with abdominal pain; the remaining LAMNs were discovered incidentally during surgery $(n=24)$, imaging $(n=10)$, or colonoscopy $(n=3)$. Seventy-five tumors $(72 \%)$ were grossly dilated, though the entire appendix was submitted in only $48(46 \%)$ cases. Lesion size ranged from 0.5 to $19.0 \mathrm{~cm}$. Most cases showed low-grade dysplasia; only one showed focal high-grade dysplasia. Thirty (29\%) demonstrated epithelial denudation (focal: 19, near-complete: 11); these cases were often markedly dilated and contained luminal microcalcifications. Thirty $(29 \%)$ had mucosal Schwann cell proliferation. Seventy-two $(69 \%)$ had some degree of appendiceal wall involvement, and $25(24 \%)$ were associated with histopathologic evidence of PP (11 with cellular mucin, 14 with acellular mucin). Four had a positive proximal margin (3 luminal epithelium, 1 luminal acellular mucin). Only one patient died of disease, and one other was alive with disease at last follow-up.

Conclusions: Previous LAMN studies have utilized both departmental and extradepartmental material; our single-institution review demonstrated lower rates of denudation and PP than some prior studies. Some LAMNs may be markedly dilated, with extensive denudation and microcalcification. Appendiceal mucosal Schwann cell proliferation has been associated with normal appendixes and appendiceal diverticula, but not previously with LAMN; evidence of this process on initial sampling should prompt further sampling to exclude a LAMN.

$700 \quad$ Metastatic Breast Carcinoma with Signet-Ring Features versus Gastrointestinal Signet-Ring Tumors: Assessment of Immunohistochemical Markers

Yiang Hui, Kara A Lombardo, Murray B Resnick, Yihong Wang. Brown University/ Rhode Island Hospital, Providence, RI.

Background: Metastatic breast carcinomas to the stomach may show signet ring features mimicking primary gastric adenocarcinomas. These cannot be differentiated by morphology. Although immunohistochemistry (IHC) is useful, relatively newer markers such as GATA- 3 have not been evaluated in this context. We aimed to assess breast carcinomas exhibiting signet ring morphology and GI primary signet-ring tumors by IHC to delineate an optimal panel for making this distinction and identify its associated pitfalls. 
Design: Primary breast and gastrointestinal carcinomas were reviewed for signet ring features. CK7, CK20, ER, PR, HER2, CDX2, e-cadherin, GATA-3, and HepPar IHC was performed. ER, PR, and HER2 studies were scored per $2013 \mathrm{ASCO} / \mathrm{CAP}$ guidelines. HER2 CISH was performed if IHC was equivocal. Statistical analysis was performed by Fisher's exact test.

Results: Forty-three cases meeting study criteria were identified. Of 20 primary breast carcinomas, 12 were ductal, 7 lobular, and 1 mucinous. Among 23 primary GI tumors, 12 were gastric. CK20, ER, PR, CDX2 and GATA-3 showed significant differences between breast and GI groups (Table 1).

\begin{tabular}{|l|l|l|l|}
\hline & Breast (\%) [n=20] & $\begin{array}{l}\text { Gastrointestinal (\%) } \\
{[\mathrm{n}=23]}\end{array}$ & \\
\hline CK7 + & $16(80)$ & $18(78)$ & $\mathrm{p}=0.99$ \\
\hline CK7 - & $4(20)$ & $5(22)$ & \\
\hline CK20 + & $2(10)$ & $18(78)$ & $\mathrm{p}<0.001$ \\
\hline CK20 - & $18(90)$ & $5(22)$ & \\
\hline E-cad + & $17(65)$ & $10(43)$ & $\mathrm{p}=0.08$ \\
\hline E-cad - & $7(35)$ & $13(57)$ & \\
\hline ER + & $16(80)$ & $0(0)$ & $\mathrm{p}<0.001$ \\
\hline ER - & $4(20)$ & $23(100)$ & \\
\hline PR + & $11(55)$ & $0(0)$ & $\mathrm{p}<0.001$ \\
\hline PR - & $9(45)$ & $23(100)$ & \\
\hline HER2 + & $4(20)$ & $1(4)$ & $\mathrm{p}=0.167$ \\
\hline HER2 - & $16(80)$ & $22(96)$ & \\
\hline CDX2 + & $1(5)$ & $20(87)$ & $\mathrm{p}<0.001$ \\
\hline CDX2 - & $19(95)$ & $3(13)$ & \\
\hline GATA-3 + & $19(95)$ & $0(0)$ & $\mathrm{p}<0.001$ \\
\hline GATA-3 - & $1(5)$ & $23(100)$ & \\
\hline HepPar + & $2(10)$ & $9(39)$ & $\mathrm{p}=0.43$ \\
\hline HepPar - & $18(90)$ & $14(61)$ & \\
\hline A & & & \\
\hline
\end{tabular}

Among breast primaries, GATA-3 was negative in a lobular carcinoma which was CK7, ER, PR, and HER2-positive. CDX2 was positive in another lobular carcinoma with gastric metastases expressing CK20, ER, PR, and GATA-3.

Conclusions: In rare cases, metastatic breast carcinomas showing signet ring features may express markers commonly found in GI primary signet ring carcinomas or lose expression of common breast markers. While relatively newer markers such as CDX2 and GATA-3 may show unexpected expression patterns, our data suggest that CK20, ER, PR, CDX2, and GATA-3 would be most effective in working up this differential in such cases.

$701 \quad$ Histopathologic Changes in the Gastrointestinal Tract During Anti-Tumor Necrosis Factor- $\alpha$ Therapy

Danielle Hutchings, Lysandra Voltaggio. Johns Hopkins School of Medicine, Baltimore, MD.

Background: Sarcoid-like granulomas in patients taking tumor necrosis factor- $\alpha$ (TNF- $\alpha$ ) therapy have been described in the lungs, lymph nodes, skin, brain and bone marrow. Additionally, there are reports of patients developing inflammatory bowel disease (IBD) while on this therapy. The aim of this study was to identify histopathologic changes in the gastrointestinal (GI) tract of patients taking anti-TNF- $\alpha$ therapy.

Design: 260 patients taking anti-TNF- $\alpha$ therapy were identified through the outpatient pharmacy. 48 had GI biopsies taken. The medical record of each patient was reviewed. 32 patients with IBD and 1 with sarcoidosis were excluded. 10 of the 16 remaining had GI biopsies performed while taking anti-TNF- $\alpha$ therapy and the histopathology was reviewed for each.

Results: Results are summarized in Table 1. The underlying condition requiring antiTNF- $\alpha$ therapy was rheumatoid arthritis in 5 patients, spondyloarthropathy in 3 , and psoriasis in 2.5 patients were taking etanercept, 4 adalimumab, and 1 certolizumab pegol. 8 patients were symptomatic and 2 underwent screening colonoscopies. Increased apoptotic bodies were seen in upper and/or lower biopsies in 6 patients ( 2 adalimumab, 4 etanercept). 3 patients on etanercept had findings other than or in addition to increased apoptosis. The first had sarcoid-like granulomas in the stomach, descending, and rectosigmoid colon, which persisted in the stomach (though poorly formed) one year after the drug was stopped. The second had active chronic inflammatory bowel disease and the third had chronic atrophic gastritis with intestinal metaplasia and prominent eosinophils.

Conclusions: These results suggest a rare but clinically significant association between anti-TNF- $\alpha$ therapy, especially etanercept, and the development GI disease, including sarcoid-like granulomas, IBD, chronic atrophic gastritis, and increased apoptosis, further contributing to the ever expanding repertoire of drug associated injury in the GI tract.

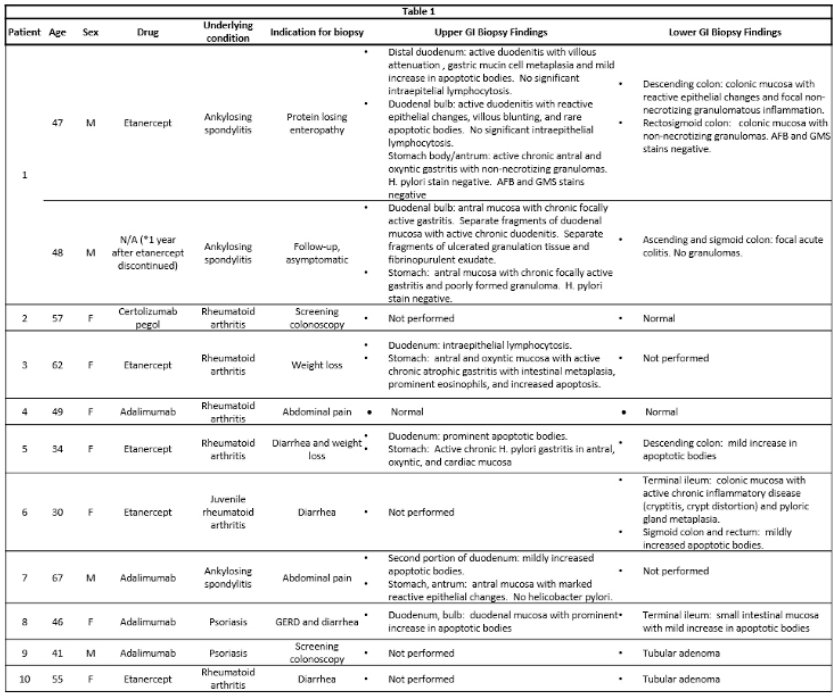

702 Mismatch Repair System Molecule Immunophenotype and BRAF V600E Genotype of PD-L1-Positive Primary and Metastatic Colorectal Carcinomas

Shingo Inaguma, Anna Felisiak-Golabek, Zengfeng Wang, Jerzy Lasota, Markku M Miettinen. Aichi Medical University, Nagakute, Aichi, Japan; National Institutes of Health, Bethesda, MD.

Background: The programmed cell death ligand-1/programmed cell death 1 (PD-L1/ PD-1) pathway is crucial for the modulation of immune responses and self-tolerance. Aberrantly expressed PD-L1 allows tumor cells to evade host immune system and is considered to be a mechanism of adaptive immune resistance. Immunohistochemical detection of PD-L1 expression is a predictor of clinical response of PD-L1-positive tumors (including colorectal carcinomas) to inhibitor treatment. The aim of this study was to characterize mismatch repair (MMR) system molecule immunophenotype and BRAF V600E genotype of primary and metastatic PDL-1-positive colorectal carcinomas (CRCs).

Design: Four hundred fifty-four primary and 189 metastatic CRCs were evaluated immunohistochemically for PD-L1 and MMR system molecule expressions. Immunohistochemistry was performed on Leica Bond-Max automatic immunostainer using 1:200 diluted anti-PD-L1 rabbit monoclonal antibody E1L3N and bond AR2 Leica protocol. A cut of-value for PD-L1 positivity was $5 \%$ of positive cell present in the sample. DNA samples from randomly selected 16 primary and 13 metastatic PDL1-positive CRCs were evaluated for BRAFV600E mutation using PCR amplification and Sanger sequencing.

Results: Fifty-four (12\%) of 454 primary CRCs revealed PDL-1 expression either on the cell membrane $(\mathrm{n}=30[7 \%])$ or in the cytoplasm $(\mathrm{n}=24[5 \%])$. Twenty-six $(14 \%)$ of 189 metastatic tumors expressed PD-L1. The cytoplasmic staining was seen in great majority of cases $(n=23[89 \%])$, while only a small fraction of metastases displayed membrane PD-L1 positivity. MMR-deficiency was detected in 40 (74\%) of 54 PD-L1positive primary CRCs and in $3(12 \%)$ of 26 PDL-1-positive metastatic tumors. BRAF V600E mutation was found in 14 (88\%) of 16 PD-L1 -positive primary CRCs and in $3(23 \%)$ of 13 PD-L1-positive metastatic tumors.

Conclusions: Primary CRCs with PD-L1 expression showed substantially higher frequency of MMR-deficiency and BRAF V600E genotype compare to previously published data of unselected CRCs. However, PD-L1-positive primary and metastatic CRCs revealed different MMR immunophenotype and BRAF V600E genotype. While majority of primary PD-L1 positive tumors displayed MMR-deficiency and BRAF V600E mutation, considerably smaller fraction of PD-L1 positive metastatic tumors showed MMR-deficiency and carried BRAF V600E mutation. Comprehensive evaluation of consequent biopsies from CRCs at the different stage of progression are necessary to pinpoint genetic and epigenetic changes responsible for this phenomenon.

703 Gastritis and Intestinal Metaplasia in Vertical Sleeve Gastrectomy: A Cause for Systematic Preoperative Screening and Followup in Bariatric Surgery Patients

Shriram Jakate, Mark Pool. Rush University Medical Center, Chicago, IL.

Background: Increasing numbers of patients with morbid obesity and associated disorders qualify for bariatric surgical interventions. Among the available types of bariatric procedures, only vertical sleeve gastrectomy (VSG) typically produces a resected gastrectomy specimen for pathological evaluation. Apart from the required metabolic, nutritional and psychological screening and followup, bariatric surgeons vary in their choice of other preoperative evaluations such as esophagogastroduodenoscopy (EGD) and upper GI series. If screening EGD is performed, gastric biopsies are not generally procured unless gastric mucosa appears abnormal. We reviewed VSG gastrectomy specimens to determine if pathological findings would influence preoperative screening and/or postoperative followup.

Design: From our clinical and pathology databases, we retrieved 301 VSG specimens (226 females and 75 males, $\mathrm{F}>\mathrm{M}=3: 1$, ages 23-65, mean age 44) in a six-year period (2011-2016). The pathology reports and slides were reviewed for specific and nonspecific 
types of gastritis, Helicobacter organisms (including review of immunohistochemical stain), intestinal metaplasia, dysplasia and other findings if present. Database was also searched for treatment for Helicobacter gastritis and followup biopsies.

Results: Active moderate to severe Helicobacter gastritis was seen in 30 patients $(10 \%$ cases), 12 of which also showed intestinal metaplasia. 174 patients $(57 \%)$ showed mild to moderate chronic gastritis with no Helicobacter organisms and 20 of these also showed intestinal metaplasia. No dysplasia or carcinoma was seen in any patient. Other than these findings, relatively few other patients showed incidental findings such as fundic gland polyps (8), autoimmune-type atrophic gastritis (1), $1.5 \mathrm{~cm}$ lipoma (1), $0.7 \mathrm{~cm}$ GIST (1) with no mitosis, and a $1.7 \mathrm{~cm}$ glomus tumor (1). Helicobacter infection was medically treated but no patient with intestinal metaplasia with or without Helicobacter gastritis (10\% of all patients) had followup biopsies.

Conclusions: Two thirds of all sleeve gastrectomy specimens $(67 \%)$ have chronic gastritis and $10 \%$ have active Helicobacter infection and/or intestinal metaplasia. These patients have no preoperative screening specifically for chronic gastritis or postoperative followup biopsy evaluation when intestinal metaplasia is associated with gastritis. Since the long-term effects of such potentially pre-neoplastic changes in the gastric remnant are uncertain, screening and postoperative followup in VSG bariatric surgery may need modifications.

\section{Tissue Transglutaminase Immunohistochemistry: A Potentially Useful Adjunct Marker for a Tissue Diagnosis of Celiac Disease in Patients with Normal Duodenal Biopsies}

Sarah Jamshed, Karen Dresser, Ninfa M Candela, Xiaofei Wang. University of Massachusetts Medical School, Worcester, MA.

Background: Duodenal biopsy is considered the 'gold standard' in the diagnosis of celiac disease. However, they are often non-specific or histologically unremarkable, providing minimal clinical information. The aim of this study is to investigate the presence of tissue transglutaminase (tTG) in the duodenal mucosa of patients with positive celiac serology and pathologically normal biopsies, and assess whether tTG immunohistochemistry is a useful tool in the diagnosis of evolving celiac disease in an otherwise normal duodenal mucosa.

Design: Cases of duodenal biopsies were retrieved from our institution's database, and divided into positive and negative controls, and test group. The positive control included those with histologic features of celiac disease and serum antibodies. Biopsies with chronic duodenitis and negative serology acted as negative control. The test group included those with clinical symptoms, circulating antibodies, and preserved villous architecture. An antibody to tTG was used; dark brown, granular, cytoplasmic staining within surface epithelial enterocytes was considered positive.

Results: Forty-seven cases were selected and the stained slides were blindly reviewed. Staining was reported as positive or negative. The positive control group included 15 cases (median age 16; M=4, $\mathrm{F}=11$ ) and demonstrated positive staining in $14 / 15$ cases (sensitivity $=93 \%$ ). Ten cases were used as negative control (median age $15.5 ; \mathrm{M}=3$, $\mathrm{F}=7$ ) and $10 / 10$ cases stained negatively (specificity $=100 \%$ ). The test group included 22 cases (median age 27.5; M=4, F=18). Positive staining was seen in 15/22 cases $(68 \%)$, with appropriate staining of surface epithelium and accompanying absence within mucosal crypts. One case (4\%) was indeterminate and six cases $(27 \%)$ were negative. Interestingly, the intensity of staining was similar between both new and advanced cases of celiac disease.

Conclusions: Our study shows high sensitivity and specificity for tTG immunohistochemistry and may be used as an ancillary study in biopsies which appear pathologically normal or with non-specific inflammatory changes. When paired with a high clinical index of suspicion, positive staining favors celiac disease over chronic duodenitis. The small sample size was the main limitation. Additionly, only new diagnoses were selected, so no follow up data was analyzed. Further studies are warranted to investigate these factors.

705 Distinguishing Hyperplastic Polyps from Small $(<1 \mathrm{~cm})$ Sessile Serrated Adenoma/Polyps - How Good Are We?

Rahul Jawale, John R Goldblum, Daniela Allende, Michael Cruise, Ilyssa Gordon, Walter Henricks, Jennifer Jeung, Keith Lai, James Lapinski, Thomas Plesec, Scott Robertson, Erica Savage, Rocio Lopez, Carol Burke, Deepa T Patil. Cleveland Clinic, Cleveland, $\mathrm{OH}$.

Background: Sessile serrated adenoma/polyps(SSP) are usually large $(>1 \mathrm{~cm})$, proximal and associated with cancer risk. Consensus recommendations endorse $\geq 1$ unequivocal abnormal shaped crypt to diagnose SSP. In addition to histology, pathologists use location and size to differentiate hyperplastic polyp(HP) from SSP. Given different surveillance implications, we performed an interobserver analysis to assess the ability of $11 \mathrm{GI}$ pathologists blinded to polyp size or location to apply consensus criteria to differentiate HPs from SSPs $(6-9 \mathrm{~mm})$.

Design: 68 polyps $<1 \mathrm{~cm}$ in size were reviewed, classified as $30 \mathrm{SSP}$ and $38 \mathrm{HP}$; clinical, endoscopic and consensus criteria were used to establish a reference diagnosis. Cases were randomized and blindly reviewed in two rounds, without and with knowledge of polyp location. Dilatation limited to upper $2 / 3$ rd of crypt, crypt base dilatation, basement membrane(BM) thickening, L/T shaped crypts, and prolapse change were recorded. Cases were analyzed as correct vs incorrect based on reference diagnosis. $\kappa$-statistics was used to assess interobserver agreement.

Results: The mean patient age was 62 yrs (40 F,28 M). 21(70\%) SSPs were right(rt) sided, $9(30 \%)$ were left(lt) sided; $12(40 \%)$ were $\leq 5 \mathrm{~mm}$ and $18(60 \%)$ were $6-9 \mathrm{~mm}$. $12(32 \%)$ HPs were rt sided, $26(68 \%)$ were lt sided, $21(55 \%)$ were $\leq 5 \mathrm{~mm}$, and $17(45 \%)$ were $6-9 \mathrm{~mm}$. Interobserver agreement was weak $(\kappa-0.41)$ in round 1 , but changed significantly to 0.5 in round $2(\mathrm{p}<.05)$ for polyps $\leq 5 \mathrm{~mm}$. When $8 / 11$ pathologists agreed(consensus diagnosis), features reported with SSP included basal dilatation( $92 \%$ ), L/T shaped crypts $(53 \%)$, and prolapse change $(18 \%)$, while dilatation of upper $2 / 3^{\text {rd }}$ of crypts( $73 \%)$, BM thickening $(22 \%)$, and prolapse change $(51 \%)$ were reported in HP. Prolapse change in HP $6-9 \mathrm{~mm}$ was the only feature that significantly changed between two rounds. A change in consensus diagnosis between two rounds was seen in $12 / 68(18 \%)$ polyps. In $8 / 12 \mathrm{rt}$ sided polyps, a correct diagnosis of HP was changed to SSP in 7 cases; while in 1 case, an incorrect diagnosis of HP was changed to SSP. In 4/12 lt sided polyps, a correct diagnosis of SSP was changed to HP in 2 cases; SSP was changed to a correct diagnosis of HP in the remaining 2 cases.

Conclusions: Classification of small serrated polyps is difficult, and is significantly influenced by location. Our results indicate a need for long term follow-up studies,and suggest reconsidering surveillance guidelines for serrated polyps $<1 \mathrm{~cm}$ in size.

\section{Recent Increase in Gastric Carcinoma and Characterization of}

\section{Gastric Polyp Burden in Western Patients with FAP}

Rahul Jawale, Gautam Mankaney, Pamela Leone, Lisa La Guardia, Margaret O'Malley, James Church, Carol Burke, Deepa T Patil, Scott Robertson, Michael Cruise. Cleveland Clinic, Cleveland, $\mathrm{OH}$.

Background: Gastric carcinomas (GC) are rarely reported in Western patients with familial adenomatous polyposis (FAP) and there are no specific surveillance recommendations for FAP related GC. We describe a recently observed increase in the incidence of GC in our FAP patients $(n=11)$.

Design: We performed clinicopathologic assessment of FAP related gastric polyps(GP) and GC of all cases of GC $(\mathrm{n}=11)$ in patients with FAP at a single-center with an inherited colorectal cancer registry $(\mathrm{n}=767)$. The standardized incidence ratio (SIR) was calculated using the SEER database of GC. GPs and GCs from 10/11 FAP patients were reviewed by pathologists for subtype, dysplasia, and associated histological features. Results: 11 cases of GC were identified with an SIR of 154. The mean age at GC diagnosis was 53.4 (25-75)yrs. The mean EGD surveillance period was 11 (4-20)yrs with surveillance interval of 5-15 months. Metastatic disease was detected in 5 patients. 161 gastric polyps from 10 patients were evaluated. There were 85 FGPs, 32 pyloric gland adenomas (PGA), 7 gastric adenoma-intestinal type (GA-IT), 29 gastric adenomafoveolar type (GA-FT) and 8 mixed polyps (MP) $(n=8) .48(56 \%)$ fundic gland polyps (FGP) were without dysplasia. 37/85 FGPs had LGD and none had HGD in a pure form. $22 / 32(69 \%)$ of PGAs had HGD, 7/22(32\%) PGA with HGD had an adenocarcinoma. All 7 GA-IT had LGD. 21/29(72\%) GA-FT had LGD, 8/29 had HGD and 1 was with adenocarcinoma. MPs $(n=8)$ all demonstrated HGD with $3 / 8$ demonstrated squamoid/ neuroendocrine phenotype and were associated with adenocarcinoma. Interestingly, 2 PGA, HGD had TSA features and 3 PGA,HGD had a lymphoid stroma.

13/161 GPs ( $8 \%$ ) had adenocarcinoma (IMC or invasive) with 3 patients having multiple tumors. 7/13 (54\%) arose in a background of pure PGA with HGD and 1 in GA-FT with HGD. 5 adenocarcinomas arose in MPs, and 4 of these polyps had PGA with HGD $(80 \%)$ and 3 with squamoid/neuroendocrine phenotype $(60 \%)$.

Conclusions: Our data demonstrates a rapid alteration in the GC spectrum in FAP in the last decade with acceleration in the last 5 years with an overall SIR of 154. FAP patients harbor a spectrum of polyps including FGP, PGA, GA-IT and GA-FT and MP. MP and PGA, HGD appear to have the greatest association with GC in FAP patients. A squamoid/neuroendocrine phenotype can be associated with MPs and co-exist with GC. GC should remain a consideration in Western patients with FAP, especially if the overall polyp burden comprises PGAs or MPs.

\section{$707 \quad$ EZH2 as a Useful Diagnostic Tool for Grading Gastrointestinal} (GI) Neuroendocrine Tumors

Rajeswari Jayakumar, Jia Qin, MA Siddiqi, Raavi Gupta. SUNY Downstate Medical Center, Brooklyn, NY; Massachusetts General Hospital, Boston, MA.

Background: Enhancer of zeste homolog 2 (EZH2) is an enzymatic subunit of polycomb repressive complex 2 which plays an important role in chromatin compaction and gene silencing, and has been shown to be increased in various high grade tumors. Increased EZH2 expression has been shown to correlate with high grade lung neuroendocrine tumors. Its expression in gastrointestinal(GI) neuroendocrine tumors has not been investigated. Here we report on its usefulness along with $\mathrm{Ki}-67$ as a marker for grading GI neuroendocrine tumors.

Design: Sixty-four cases of gastrointestinal and pancreatic neuroendocrine tumors of grade $1(\mathrm{~N}=39)$, grade $2(\mathrm{~N}=17)$ and grade $3(\mathrm{~N}=8)$ were retrieved and re-evaluated. Immunostaining for EZH2 and Ki-67 was performed (Ventana Medical Systems, Tucson, AZ), and percent positive cells were enumerated. Mitosis/10 high power fields was recorded. The data were analyzed by 2-tailed Mann-Whitney U test between the different tumor grades, and by Pearson correlation between EZH2, Ki-67 and mitosis. $P$ value of $<0.05$ was considered statistically significant.

Results: EZH2 expression was significantly high in G3 than G1 $(\mathrm{P}<0.0001)$ and G2 $(\mathrm{P}=0.0001)$. There was no significant difference in $\mathrm{EZH} 2$ expression between $\mathrm{G} 1$ and $\mathrm{G} 2(\mathrm{P}=0.9093)$. Differences between tumor grades with respect to $\mathrm{Ki}-67$ and mitosis were all significant $(\mathrm{P}=0.0001)$. Correlations between $\mathrm{EZH} 2$ and $\mathrm{Ki}-67, \mathrm{EZH} 2$ and mitosis, Ki-67 and mitosis were all statistically significant $(\mathrm{P}<0.0001)$. Ki-67 stained the infiltrating lymphocytes while EZH2 stained only the tumor cells.

\begin{tabular}{|c|c|c|c|}
\hline \multirow{2}{*}{ Tumor Grade } & \multicolumn{2}{|c|}{$\begin{array}{c}\text { Percent cells positive } \\
\text { Mean (Range) }\end{array}$} & \multirow{2}{*}{ Mitosis/10 HPFMean (Range) } \\
\cline { 2 - 3 } & EZH2 & Ki-67 & \\
\hline G1 $(\mathrm{n}=39)$ & $1(0-2)$ & $1.3(0-2)$ & $0.46(0-1)$ \\
\hline G2 $(n=17)$ & $1.2(0-6)$ & $4.2(0-11)$ & $3.3(0-10)$ \\
\hline G3 $(n=8)$ & $63(40-80)$ & $45.5(21-70)$ & $25.8(18-36)$ \\
\hline
\end{tabular}


Conclusions: Increased EZH2 expression correlates with Ki-67 expression and mitosis in GI neuroendocrine tumors of grades 1 through 3. Hence, EZH2 expression can be used as an additional diagnostic marker in GI neuroendocrine tumors. Relative to Ki-67, lack of EZH2 expression in lymphocytes is diagnostically advantageous. As in other cancers, EZH2 can be used as a potential therapeutic target in neuroendocrine tumors.

708 Tumor Markers

William Richard Jeck, Kshitij S Arora, Elena Brachtel, Vikram Deshpande. Massachusetts General Hospital, Boston, MA.

Background: Immunohistochemical and in-situ hybridization markers of tumor type are invaluable in unwinding the diagnosis of poorly-differentiated and histologically similar tumors. To identify superior markers, we analyzed The Cancer Genome Atlas (TCGA) RNAseq data to find genes with highly elevated expression in specific tumor types.

Design: We analyzed normalized RNAseq data over 10,000 tumors from 33 tumor types across 20533 quantified genes from TCGA archives. We first analyzed expression of existing markers to confirm that RNA expression differences recapitulate known immunohistochemical staining patterns. We next identified genes maximizing the receiver-operator curve with respect to a specific tumor type. Finally, we sought to confirm selected markers by RNA in-situ hybridization assay in tissue microarrays from six tumor types: 28 breast carcinomas, 12 hepatocellular carcinomas, 22 colorectal carcinomas, 19 cholangiocarcinomas, 22 esophageal adenocarcinomas, and 46 pancreatic ductal adenocarcinomas.

Results: TCGA RNAseq expression profiles for well-known markers matched known differential IHC staining (e.g. KER7, KER20, WT1, ARG1, MITF, PAX8, GATA3, SYN, and KIT), with a few notable exceptions (e.g. TTF1). Our analysis for novel markers produced hundreds of potential gene targets. In-situ hybridization testing was used for two selected markers: ABOB (Apolipoprotein B, a marker for HCC), and PRLR (Prolactin Receptor, a marker for breast cancer). APOB stained $100 \%$ of HCCs and $68 \%$ of cholangiocarcinomas, with some off-target staining in 2 of 22 esophageal adenocarcinomas $(9 \%)$, without staining in other tumors. APOB did not stain bile duct carcinomas, instead staining exclusively intrahepatic cholangiocarcinoma. PRLR proved a highly specific marker, staining $46 \%$ of breast cancers, but none of the other tumor types.

Conclusions: We find that TCGA RNAseq data is a useful tool for markers development. Additional optimization of the informatics and ISH techniques may yield powerful markers for identification of tumor origin. Expansion of the base RNAseq data set to include rare tumors and metastatic specimens could further widen the usefulness and validity of this approach

\section{Mismatch Repair Deficiency (MMR-D) and Programmed Death} Ligand-1 (PD-L1) Expression in Gastric Adenocarcinomas

Taylor M Jenkins, Rashmi Tondon, Li-Ping Wang, Amy Ziober, Priti Lal, Stuti G Shroff, Emma E Furth, Kristen M Stashek. University of Pennsylvania, Philadelphia, PA Background: Immunohistochemical staining (IHC) for MMR (MLH1, MSH2, MSH6, and PMS2) is not routinely performed on gastric adenocarcinoma (GA) as is commonly done in colon cancers, in which microsatellite instability and its high mutational frequency resulting in neo-antigens correlates with high expression of PDL1. The clinical and pathologic features of MMR-deficient (MMR-D) GA are not well known. The goals of our study were to assess the prevalence of MMR-D in GA and its correlation with PD-L1 expression, whose expression offers an immunotherapy target with checkpoint inhibitors.

Design: 52 patients with GA who underwent resection from 1/12-6/16 were reviewed and categorized as diffuse or intestinal-type. Tissue microarrays were constructed with each tumor cored in triplicate. IHC (with adequate controls) for the MMR proteins, PD-L1 and in-situ hybridization for Epstein-Barr (ISH-EBV) were performed. ISH-EBV and MMR stains were categorized as positive/retained versus negative/absent. PD-L1 expression was considered absent if $<1 \%$ of cells showed partial/complete membranous staining, low expression if $1-49 \%$ showed staining, and high if $>49 \%$ showed staining. Results: See tables 1-2. Chi-square/T-test were applied.

\begin{tabular}{|l|c|c|}
\hline & Intestinal $(\mathrm{n}=38)$ & Diffuse $(\mathrm{n}=14)$ \\
\hline Age (avg) (range) & $70(40-92)$ & $60(37-81)$ \\
\hline Sex (\% male) & $55 \%$ & $29 \%$ \\
\hline MMR deficient & $26 \%(10 / 38)$ & $0(0 / 14)$ \\
\hline EBV positive & $5 \%(2 / 38)$ & $7 \%(1 / 14)$ \\
\hline High PD-L1 expression & $11 \%(4 / 38)$ & $0(0 / 14)$ \\
\hline NGS data (n=8) & Normal (1), FBXW7 (1), & TP53 (3), Normal (1), \\
\hline Syndromes & CTNNB1/GNAS/KRAS (1) & ATM \\
\hline
\end{tabular}

\begin{tabular}{|l|c|c|c|}
\hline \multicolumn{4}{|c|}{ Clinical and Pathologic Features of Intestinal-Type GA (n=38) } \\
\hline & NOS (n=26) & MMR deficient (n=10) & EBV pos (n=2) \\
\hline Age (avg) (range) & $68(40-87)$ & $75(61-92)$ & $77(72-82)$ \\
\hline Sex (\% male) & $62 \%$ & $40 \%$ & $50 \%$ \\
\hline PD-L1 expression & $88 \%(23 / 26)$ & $30 \%(3 / 10)$ & $50 \%(1 / 2)$ \\
\hline -Negative & $8 \%(2 / 26)$ & $50 \%(5 / 10)$ & 0 \\
\hline -Low (1-49\%) & $4 \%(1 / 26)$ & $20 \%(2 / 10)$ & $50 \%(1 / 2)$ \\
\hline -High (>50\%)
\end{tabular}

Conclusions: The majority of GA cases were intestinal type (73\%). Of these, MMR-D was noted in $26 \%$ and had higher frequencies of PD-L1 expression ( $p>.001)$ compared to proficient cases, with only one case showing high expression (4\%). The remaining cases were diffuse-type (27\%) and none showed loss of MMR or high PD-L1 expression. In summary, MMR-D intestinal-type GA has higher frequencies of PD-L1 expression and may represent a potential immunotherapy target with PD-1 inhibitors.

710 IBD Specific Phenotypes of Colorectal Carcinomas Are Associated with Unique Molecular Alterations

Melanie E Johncilla, Neal I Lindeman, Mikhail Lisovsky, Amitabh Srivastava. Brigham and Women's Hospital, Boston, MA; Dartmouth-Hitchcock Medical Center, Lebanon, $\mathrm{NH}$

Background: Patients with inflammatory bowel disease (IBD) are at increased risk for colorectal cancer (CRC). IBD-associated adenocarcinomas (IBD-AA) may resemble conventional adenocarcinoma (ACA) but may also show a spectrum of phenotypes seldom seen in sporadic CRC. These include low grade tubuloglandular adenocarcinomas (LGTA), ACA arising in hypermucinous dysplasia, and well differentiated ACA rich in goblet cells or gastric type mucin. Our aim was to determine whether these unique IBD-AA are associated with molecular alterations distinct from sporadic CRC.

Design: Inclusion criteria for IBD-AA were: ACA with the distinct features mentioned, those arising in a non-adenoma like DALM or those with multifocal dysplasia. Carcinomas with features resembling sporadic CRC were excluded unless one of the latter two criteria were fulfilled. DNA was extracted from $10 \mathrm{4u}$ slides from FFPE blocks. Illumina TruSeq ${ }^{\mathrm{TM}}$ was used to prepare the library for sequencing the exons of a custom 300 gene panel using an Illumina HiSeq 2000.

Results: 15 tumors met our criteria for IBD-AAs. Patient age ranged from 31-93 yrs (median:61). Six of 15 IBD-AAs showed a gastric phenotype similar to upper GI tract adenocarcinomas, 3 were associated with hypermucinous dysplasia, and 1 was a LGTA. Two ACA with serrated dysplasia, two mucinous ACA and 1 conventional CRC were associated with either multifocal dysplasia or a non-adenoma like DALM The number of mutations per case ranged from $1-53$ (median: 7). The most frequent mutations were TP53 (11/15) spread across the entire morphological spectrum of IBDAA. CDKN2A (3/15), MECOM (2/15) and IDH1 (2/15) were some low frequency mutations seen. ACA with a gastric phenotype showed TP53 (4/6), CDKN2A (2/6), $\operatorname{ARID1A}(1 / 6)$ and $\mathrm{CDH} 1(1 / 6)$, mutations similar to those in gastric carcinomas. ACA in hypermucinous dysplasia had TP53 (2/3) and ARID1B (1/3) mutations and one had an IDH1 R132C mutation, described in cholangiocarcinomas. The conventional CRC had few mutations similar to sporadic CRCs including TP53 and ARID1a. The two mucinous CRC had no KRAS or BRAF mutations suggesting that these tumors may be distinct from conventional mucinous ACA.

Conclusions: TP53 mutations are highly prevalent in IBD-AA irrespective of histological tumor type and grade. IBD-AA that show a gastric phenotype also harbor molecular alterations similar to those seen in upper GI tract ACA. These findings have implications for neoplasia diagnosis and treatment for patients with $\mathrm{CRC}$ in the setting of IBD.

711 Identification of Unique Mutant Gene Profiles and Morphologic Features in a Large Cohort of BRAF-Mutant Colon Adenocarcinomas Using Next Generation Sequencing Approach

Ryan D Jones, David Dittmann, Nike T Beaubier, Juehua Gao, Guang-Yu Yang. Northwestern University, Chicago, IL.

Background: Although only approximately $10 \%$ of colorectal cancers (CRC) carry BRAF mutations, they harbor a worse prognosis with aggressive behavior. BRAFmutation essentially excludes the possibility of Lynch Syndrome, but these commonly lose MLH1 mismatch repair protein likely due to sporadic promoter methylation. Whether other cancer-related gene alterations in collaboration with mutant BRAF leads to carcinogenesis is not well known. This study utilizes next generation sequencing (NGS) together with immunohistochemistry (IHC) to analyze the mutation profile of cancer-related genes, expression of mismatch repair proteins, and morphology to characterize the key molecular and morphologic features in BRAF-mutant CRC. Design: Through searching the large clinical CRC cohort from Northwestern Memorial Hospital (NMH) since 2014 (total 376 cases), 45 BRAF-mutant CRC were identified using the standard clinical approaches of V600E allele-specific PCR or NGS. All of these were further analyzed for key cancer-related gene mutation profile using NGS approach. Immunohistochemical stains were performed for MLH1, PMS2, MSH2, and MSH6 protein expression with proper controls.

Results: In our NMH cohort, the frequency of BRAF-mutant CRC was $12.0 \%(45 / 376)$. Among these 42/45(93.3\%) were BRAF p.V600E and 3/45(6.7\%) were non-p.V600E including p.N5815, p.G606V, and p.D594G. Concomitant mutations in other cancerrelated genes include 16 TP53 (35.6\%), 9 PIK3CA (20\%), 4 SMAD4 (8.9\%), one KRAS codon 14 mutation, and one NRAS. 48.9\%(22/45) BRAF-mutant CRC exhibited loss of MLH1 and PMS2, with higher trends higher in cases with p53 WT (16/29, 55.2\%) compared with p53-mutations (6/16, 37.5\%). Morphologic analysis showed 82\%(37/45) were right-sided, $89 \%(40 / 45)$ were high-grade (moderately to poorly differentiated), and $34 \%(15 / 45)$ had mucinous features.

Conclusions: Our results indicate that in addition to the common p.V600E mutation, non-p.V600E mutations are present in CRC and the frequency of BRAF mutation in $\mathrm{CRC}$ is $12.0 \%$. The cancer-related gene mutation profile and IHC demonstrate that 1 ) concomitant mutations in p53, PIK3A and SMAD4 are frequently identified, 2) first report on concomitant mutations in KRAS codon 14 and NRAS, and 3) loss of MLH1 expression is frequent but not as high as literature reports. All of these data imply BRAF mutation together with either loss MLH1 or other key gene mutations (P53, PIK3A and SMAD4) are crucial for driving carcinogenesis and targeting these mutations would be significant for developing therapeutic approaches. 
712 Mucin Rich Variant of Traditional Serrated Adenoma (MrTSA): A Distinct Morphological Variant

Sangeetha N Kalimuthu, Stefano Serra, Sara Hafezi-Bakthiari, Richard Colling, Lai Mun Wang, Runjan Chetty. UHN, Toronto, ON, Canada; OUH, Oxford, Oxfordshire, United Kingdom.

Background: Traditional serrated adenomas (TSAs) account for $\sim 5 \%$ of serrated polyps, typically characterised by: exophytic villiform architecture, eosinophilic cells with brush border, indented, flat-topped luminal serrations. However, a small subset has been observed to have a mucin-rich (Mr) component. We aimed to perform a clinicopathological correlation to determine if MrTSA has unique features and ascertain differences with classic TSA (cTSA).

Design: A total of 156 TSAs were retrieved from the archives of 2 Pathology departments (UHN, Toronto and Oxford) from 2010-2016. Patient demographics and site were documented and 16 morphologic variables were evaluated including growth pattern, frequency of ectopic crypt foci (ECF), concurrence with other polyps, type of serration, presence of dysmaturation/dystrophic goblet cells (GCs) or other secretory cells, degree of inflammation and type/degree of dysplasia. TSAs with $50 \% />\mathrm{GCs}$ (GC:eosinophilic absorptive cell ratio= at least 1:1) were arbitrarily classified as MrTSAs. The number of ECFs were quantified as low (1-10/x20 magnification field [MF]) and high (>10/x20MF). Results: Of 156 TSAs, 23 fulfilled the criteria of MrTSA. More males had MrTSAs $(65 \%)$ than cTSAs $(55 \%)$ but no age difference noted. Whilst both groups showed a predilection for the left colon, MrTSAs were more frequent in the right colon $(39 \%)$ compared to cTSA $(10 \%),(p=0.012)$. Five MrTSAs $(22 \%)$ and 70 cTSAs $(52 \%)$ were associated with other polyp types, respectively. Conventional adenomatous dysplasia was present in 4/23 MrTSAs (low grade= 3; high grade=1). Distinctive morphologic features of MrTSA were variable growth pattern (endophytic [9\%], mixed $[30 \%]$,villiform/exophytic [61\%]) and significantly lower frequency of ECFs $(61 \%)$ than cTSA $(4 \%),(\mathrm{p}=0.001)$. However, all MrTSAs uniformly retain characteristic luminal serrations, at least focally. Also, inflamed endophytic MrTSAs can simulate inflammatory polyps and mimic hamartomatous polyps when there is a predominance of GCs $(>90 \%)$.

Conclusions: Our study highlights MrTSA as a distinct morphologic variant of TSA, characterized by a miscellany of features including $>50 \% \mathrm{GCs}$, fewer ECF $(<10 / \mathrm{x} 20 \mathrm{MF})$ but with preservation of archetypal luminal serrations. The biological significance of this variant is unknown; however, disruptions in Wnt signalling have been documented in TSAs and concurrent inactivation in Notch signalling may result in abnormal proliferation of GCs. Awareness of this variant is crucial to avoid misdiagnosis, given their association with aggressive type of colorectal cancer.

713 Complete Histopathologic Examination of Prophylactic Gastrectomy Specimens for CDH1 Germline Mutation: Is It Warranted in Routine Clinical Practice?

Dipti M Karamchandani, Zhaohai Yang. Penn State Hershey Medical Center, Hershey, PA.

Background: Hereditary diffuse gastric cancer (HDGC) is an autosomal dominant familial cancer syndrome. Germline CDH1 (E-cadherin) mutation is detected in $25-40 \%$ of tested families. Some prior studies performed complete histologic examination of these gastrectomies in an attempt to characterize the microscopic lesions which may have implications for targeted endoscopic surveillance biopsies. However, no prior study has questioned the utility of histologic examination of the entire specimen in routine clinical practice. Presently, there is no uniform protocol regarding the extent of histopathologic examination and many academic institutions submit the entire stomach In this study, we question the utility of complete histologic examination of the stomach in HDGC patients in routine clinical practice.

Design: A retrospective review of total gastrectomy specimens performed for $\mathrm{CDH} 1$ mutations from 2000-2015 revealed 5 prophylactic gastrectomies: 3 were entirely submitted with mapping and 2 (performed in 2004) were partially submitted. A complete re-review of all available slides for these cases was performed.

Results: No gross lesions were identified in any of the 5 specimens. The results of completely submitted specimens are tabulated in Table 1 .

\begin{tabular}{|l|l|l|l|l|l|l|l|}
\hline & Age & Sex & $\begin{array}{l}\text { Total pieces; } \\
\text { blocks }\end{array}$ & Pathology & $\begin{array}{l}\text { Foci in fundus/ } \\
\text { upper body }\end{array}$ & $\begin{array}{l}\text { Foci in mid- } \\
\text { lower body }\end{array}$ & $\begin{array}{l}\text { Foci in } \\
\text { antrum }\end{array}$ \\
\hline 1 & 33 & F & $510 ; 204$ & In situ CA & 6 & 0 & 0 \\
\hline 2 & 21 & F & $453 ; 199$ & IMC & 27 & 8 & 0 \\
\hline 3 & 53 & F & $430 ; 170$ & IMC & 6 & 0 & 0 \\
\hline
\end{tabular}

(F-female, IMC-Intramucosal carcinoma, CA- carcinoma).

For the 2 patients ( 35 year old male, 32 year old female) with incompletely submitted specimens (20 pieces and 25 pieces), 9 and 7 foci of IMC respectively were found in fundus and body and none were seen in the antrum .Further characterization as to the exact site of the body could not be delineated.

Conclusions: The patients with prophylactic gastrectomy in our series had multiple microscopic foci of in situ and/or IMC predominantly in the fundus and upper body. Noticeably, no foci in antrum were seen. No submucosal or deeper invasion was seen in any of the above specimens. The results suggest that for routine clinical practice, entire submission of prophylactic gastrectomy in HDGC patients is probably not justified given the time and cost, and especially given that there is no change in tumor stage and further clinical management. We propose to target the fundus/upper body to get maximum number of clinically significant lesions.
714 Gastric Type (Pyloric/Foveolar Gland) Adenomas of the Duodenum: A Clinicopathologic Analysis of 11 Cases

Juliana O Kissiedu, Bahar Memis, Takashi Muraki, Alyssa Krasinskas, Michelle D Reid, Yue Xue, Volkan Adsay. Emory Uni, Atlanta, GA.

Background: Gastric-type adenomatous lesions especially those of "pyloric gland type" are gaining increasing recognition. However, there is limited data regarding these lesions in the duodenum.

Design: Clinicopathologic characteristics of 11 cases of pyloric/foveolar type adenoma occurring in the duodenum were investigated.

Results: The median age was 72 with a marked female predominance(F:M 9:2). The original diagnosis was either pyloric gland or foveolar/pyloric adenoma with a median size of $1 \mathrm{~cm}($ largest, $2.3 \mathrm{~cm}$ ). Histologically, the tumors were predominantly composed of medium sized glands with undulating contours, pale cytoplasm and basally localized nuclei. Some cases had round nuclei, visible nucleoli and more acidophilic cytoplasm creating an oncocytic-like cytology. Brunner gland-like units were focally observed in 6 . No squamous morules or underlying gastric heterotopia was observed. 4 had high-grade dysplasia(HGD), depicted by basophilic foci of monotonous cells with larger nuclei and higher N/C ratio. Some HGD lesions had a papillary-like configuration. One case had architectural complexity(cribriform/solid/irregular-papillary) growth along with minimal invasive carcinoma in the lamina propria (Figure 1).

IHC with gastric markers revealed gastric-mucosal-type pattern in some (MUC6 highlighting basal to mid aspect of the mucosa and MUC5AC more on the surface). 3 cases showed prominent inverted growth with diffuse co-expression of both MUC5AC(foveolar) and MUC6(pyloric). MUC1 was seen in only 1 case(1 case with HGD and invasion). Beta-catenin nuclear staining or p53 over-expression was not observed. Follow up was available for 8 patients with a median of 38 months and no invasive adenocarcinoma of duodenum was reported.

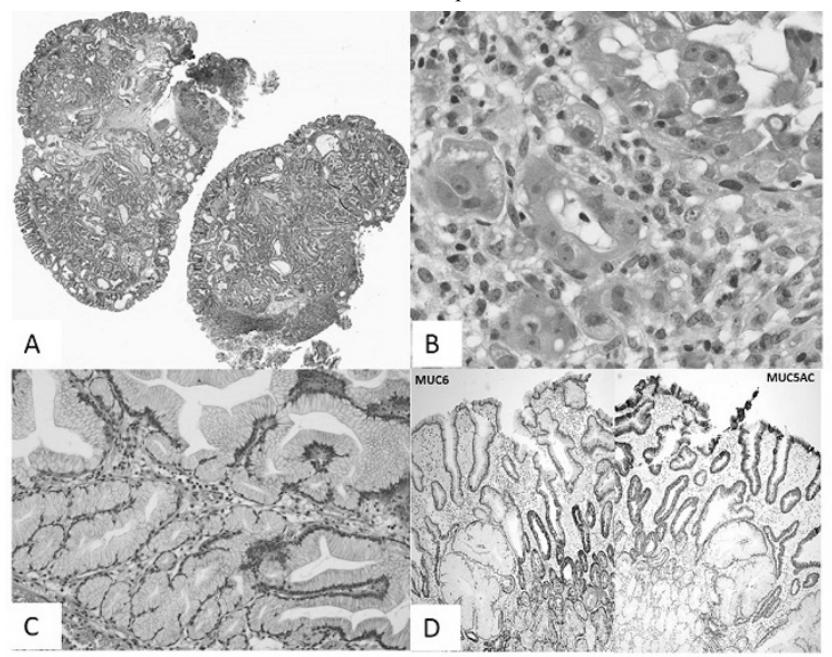

Figure 1: Pyloric/foveolar gland adenoma of the duodenum with inverted architecture(A). Area of focal invasion with oncocytic-like features (B). Pyloric/foveolar gland adenoma of the duodenum with both foveolar and pyloric glands (C). MUC5AC and MUC6 staining in pyloric/foveolar gland adenoma of the duodenum (D)

Conclusions: Gastric-type (pyloric/foveolar gland) adenomas of the duodenum are seen in elderly patients suggesting they may be a result of senescence. They show striking female predilection $(80 \%)$, suggesting a hormonal connection, but no beta-catenin nuclear stain or morule formation was identified. They show diffuse MUC5AC/MUC6 co-expression and a distinctive foveolar like cytology with more acidophilic cytoplasm and some with oncocytoid features. HGD is detected in $25 \%$, and invasion may be seen. There was no gastric heterotopia associated with these lesions.

\section{Utility of NKX6.1 Staining in Gastrointestinal and Pancreatic} Neuroendocrine Tumors

Juliana O Kissiedu, Brian Robinson, Shishir Maithel, Alyssa Krasinskas. Emory University Hospital, Syracuse, NY.

Background: NKX6.1 has been shown to be expressed more often in pancreatic and duodenal neuroendocrine tumors (NETs) than those from other sites with a sensitivity of $82 \%$ and $67 \%$ respectively, and a specificity of $93 \%$. Because we anecdotally noted NKX6.1 positivity in NETs from other sites in the gastrointestinal (GI) tract, we chose to examine the utility of this stain in our institution.

Design: NKX6.1 immunostaining was performed on tissue micro arrays (TMAs) and whole slides. Nuclear staining was scored on intensity $(0-3)$. Tumors with $\geq 5 \%$ nuclear positivity of any intensity for NKX6.1 were diagnosed as positive.

Results: 206 cases were evaluated, consisting of 110 pancreatic, 16 duodenal, 56 small bowel, 9 stomach, 9 colonic and 6 rectal NETs. NKX6.1 was more likely to be positive in pancreatic and duodenal NETs $(\mathrm{p}<0.001)$ than in NETs from other GI sites (see Table 1). The sensitivity of NKX6.1 as a marker for pancreatic and duodenal NETs, however, was $72 \%$, with a specificity of $75 \%$. 


\begin{tabular}{|l|l|l|l|}
\hline \multicolumn{4}{|l|}{ Table 1: Dristribution of NKX6.1 Staining. } \\
\hline Site of NET & $\begin{array}{l}\text { Total number of } \\
\text { Cases }\end{array}$ & $\begin{array}{l}\text { Total number of cases with } \geq 5 \% \\
\text { NKX6.1 Stain }\end{array}$ & \% Positive \\
\hline Pancreas & 110 & 78 & 70 \\
\hline Duodenum & 16 & 13 & 81 \\
\hline Small Bowell & 56 & 9 & 16 \\
\hline Stomach & 9 & 4 & 44 \\
\hline Colon & 9 & 5 & 56 \\
\hline Rectum & 6 & 2 & 33 \\
\hline & & & \\
\hline $\begin{array}{l}\text { Pancreas and } \\
\text { Duodenum }\end{array}$ & 126 & 91 & 72 \\
\hline Others & 80 & 20 & 25 \\
\hline
\end{tabular}

Conclusions: While NKX6.1 is expressed more commonly in pancreatic and duodenal NETs compared to NETs from other GI sites, the low sensitivity and specificity in our hands raises concern for the utility of this marker in determining the origin of a NET when the primary site is unknown.

716 Immunohistochemistry Based Molecular Classification of Gastric Cancer and Its Prognostic Significance

Jiwon Koh, Keun-Wook Lee, Soo Kyung Nam, Do Joong Park, Hyung-Ho Kim, Woo Ho Kim, Hye Seung Lee. Seoul National University College of Medicine, Seoul, Republic of Korea; Seoul National University Bundang Hospital, Seongnam-si, Gyeonggi-do, Republic of Korea.

Background: Gastric cancer (GC) is a heterogeneous disease entity, and substantial amount of efforts have been done to develop the classification of GC based on molecular biologic basis. However, analyzing the genomic signature is not always feasible, thus we aimed to i) validate a practical IHC based molecular classification of GC, and ii) assessed the Her2 status according to the classification.

Design: A total of 933 consecutive GC patients from two individual cohorts $(\mathrm{N}=535$ and 398, respectively) were classified into five groups as follows, using EBV in situ hybridization, MSI testing and IHC for E-cadherin and p53: group 1, EBV-positive gastric; group 2, MSI-high; group 3, MSS/EMT-like; group 4, MSS/non-EMT-like/ p53-IHC+; group 5, MSS/non-EMT-like/p53-IHC-. Her2 status was defined according to the results of Her2 IHC and SISH.

Results: The proportions of each group in whole study population are as follows: group $1,8.84 \%$; group 2, $8.61 \%$; group $3,13.20 \%$; group $4,28.08 \%$; group $5,41.28 \%$. There was no significant discrepancy of the proportions between two cohorts. On survival analysis, group 2 had the best prognosis, sequentially followed by group 5 , group 1 , group 4 , and group 3 in both study populations $(P<0.001)$. Her 2 positivity was observed in $6.9 \%$ of total population, and $93.1 \%$ of Her 2 positive patients were in group 4 and 5 , implying the significant propensity toward non-EMT phenotype.

Conclusions: We have found the close association between Her2 status and non-EMT phenotype, and proved the significant prognostic implication of IHC based molecular classification of GC.

\section{YAP1 Is Commonly Overexpressed in FAP-Related Neoplasia} Amanda M Kraus, Evelyn Carolina Polanco, Christopher Hartley, Catherine Hagen. Medical College of Wisconsin, Milwaukee, WI.

Background: Yes associated protein-1 (Yap1), a regulator of the Hippo signaling pathway, is overexpressed in various neoplasms. The APC gene normally suppresses both Yap1 and $\beta$-catenin, limiting uncontrolled cellular proliferation. Patients with Familial Adenomatous Polyposis (FAP) harbor autosomal dominant mutations in the APC gene leading to the development of thousands of colonic adenomas, as well as extracolonic neoplasms. Our aim was to evaluate Yap1 expression in a variety of lesions from FAP patients, compare to non-FAP control cases, and correlate with $\beta$-catenin expression.

Design: Lesional tissue from 11 FAP patients (4 intestinal carcinomas, 7 colonic tubular adenomas (cTA), 4 desmoid tumors, 4 fundic gland polyps (FGP), and 4 small bowel adenomas (sbTA)), and 10 non-FAP patients ( 3 cTA, 4 FGP, and 4 sbTA) was examined. Normal tissue from FAP patients was also evaluated. IHC for Yap1 (H-125; sc-15407; Santa Cruz Biotechnology) was scored for negative, low, and high nuclear and/or cytoplasmic staining as described in prior studies. $\beta$-catenin IHC was evaluated for the presence of nuclear staining.

Results: In normal tissue from FAP patients, nuclear YAP1 staining was seen predominantly in colonic crypt bases, small bowel villi, and gastric pits. No FAP normal tissue showed high cytoplasmic YAP1 staining (HI cyto). All FAP desmoid tumors and intestinal carcinomas showed high nuclear YAP1 staining (HI nuc). HI nuc was common in lesional tissue from both FAP $(91.3 \%)$ and non-FAP cases $(100 \%)$. HI cyto was more common in sbTA in non-FAP patients ( $50 \%$ vs. $0 \%$ ) (Table 1$)$.

\begin{tabular}{|l|l|l|l|l|l|l|}
\hline & \multicolumn{2}{|c|}{ HI cyto YAP1 } & \multicolumn{2}{c|}{ HI nuc YAP1 } & \multicolumn{2}{c|}{ Nuclear $\beta$-catenin } \\
\hline & FAP & Non-FAP & FAP & Non-FAP & FAP & Non-FAP \\
\hline cNormal & $0 / 3(0 \%)$ & $\mathrm{n} / \mathrm{a}$ & $3 / 3(100 \%)$ & $\mathrm{n} / \mathrm{a}$ & $0 / 3(0 \%)$ & $\mathrm{n} / \mathrm{a}$ \\
\hline sbNormal & $0 / 3(0 \%)$ & $\mathrm{n} / \mathrm{a}$ & $2 / 3(67 \%)$ & $\mathrm{n} / \mathrm{a}$ & $0 / 3(0 \%)$ & $\mathrm{n} / \mathrm{a}$ \\
\hline gNormal & $0 / 3(0 \%)$ & $\mathrm{n} / \mathrm{a}$ & $2 / 3(67 \%)$ & $\mathrm{n} / \mathrm{a}$ & $0 / 3(0 \%)$ & $\mathrm{n} / \mathrm{a}$ \\
\hline sbTA & $0 / 4(0 \%)$ & $2 / 4(50 \%)$ & $3 / 4(75 \%)$ & $4 / 4(100 \%)$ & $2 / 4(50 \%)$ & $1 / 4(25 \%)$ \\
\hline FGP & $4 / 4(100 \%)$ & $\begin{array}{l}4 / 4 \\
(100 \%)\end{array}$ & $4 / 4(100 \%)$ & $4 / 4(100 \%)$ & $0 / 4(0 \%)$ & $0 / 4(0 \%)$ \\
\hline cTA & $0 / 7(0 \%)$ & $0 / 3(0 \%)$ & $6 / 7(85.7 \%)$ & $3 / 3(100 \%)$ & $\begin{array}{l}1 / 7 \\
(14.3 \%)\end{array}$ & $\begin{array}{l}1 / 3 \\
(33.3 \%)\end{array}$ \\
\hline Desmoid & $3 / 4(75 \%)$ & $\mathrm{n} / \mathrm{a}$ & $4 / 4(100 \%)$ & $\mathrm{n} / \mathrm{a}$ & $\begin{array}{l}4 / 4 \\
(100 \%)\end{array}$ & $\mathrm{n} / \mathrm{a}$ \\
\hline $\begin{array}{l}\text { Intestinal } \\
\text { CA }\end{array}$ & $3 / 4(75 \%)$ & $\mathrm{n} / \mathrm{a}$ & $4 / 4(100 \%)$ & $\mathrm{n} / \mathrm{a}$ & $2 / 4(50 \%)$ & $\mathrm{n} / \mathrm{a}$ \\
\hline Total & $\begin{array}{l}10 / 32 \\
(31.3 \%)\end{array}$ & $\begin{array}{l}6 / 11 \\
(54.5 \%)\end{array}$ & $\begin{array}{l}28 / 32 \\
(87.5 \%)\end{array}$ & $\begin{array}{l}11 / 11 \\
(100 \%)\end{array}$ & $\begin{array}{l}9 / 32 \\
(28.1 \%)\end{array}$ & $\begin{array}{l}2 / 11 \\
(18.2 \%)\end{array}$ \\
\hline \multicolumn{7}{|c|}{ cNormal=normal colon, sbNormal=normal small bowel, gNormal=normal stomach } \\
\hline
\end{tabular}

Conclusions: YAP1 is commonly overexpressed in various neoplastic lesions from FAP patients including intestinal carcinoma and desmoid tumors. YAP1 could potentially serve as a valuable biomarker in FAP-related neoplasia, but larger studies are needed to evaluate the trends noted here.

718 The Clinical Significance of Active Colitis Following the Advent of Immunomodulator Therapy and Availability of PCR Based Microbiological Detection Panels

Heewon Kwak, Scott Matushek, Vera Tesic, Shu-Yuan Xiao, John Hart, Namrata Setia. University of Chicago, Chicago, IL.

Background: Active colitis is a morphologic pattern characterized by neutrophils in the lamina propria, neutrophilic cryptitis, or neutrophilic crypt abscesses in the absence of unequivocal chronic changes. Prior studies report infection as the most common cause of this pattern, followed by other entities: drug induced mucosal injury, incidental findings in screening colonoscopy, ischemia, and inflammatory bowel disease (IBD). Recently, there is an increased use of immunomodulators and the development of PCR based microbiologic panels (e.g. FilmArray ${ }^{\circledR}$ Gastrointestinal panel), which can simultaneously detect various bacterial, parasitic, and viral pathogens. This retrospective study updates the clinical significance of biopsies diagnosed as "mild colitis".

Design: Reports with a diagnosis of "colitis" were retrieved from our database from 2/2016, when a PCR infectious panel was first offered at our institution, to 9/2016. These cases were divided into active colitis or chronic colitis. Data comprising clinical symptoms, endoscopic findings, pertinent medication history, microbiologic PCR panel concurrently performed during the endoscopy (57) or resection (3), and follow up data up until resolution of their symptoms were reviewed on cases with active colitis without chronicity, when applicable.

Results: Of 560 cases diagnosed as "colitis", 60 had colitis without unequivocal changes of chronicity (11\%). Partially treated IBD comprised the largest subset $(23 \%)$ of active colitis cases [CD 7, ulcerative colitis (UC) 7], and evolving CD (2) and UC (2). Drugs comprised $17 \%$ of cases. Immunomodulators (e.g. Tremelimumab, Pazopanib, Ipilimumab) were more common than NSAID related cases (6 versus 2). Concurrent infectious PCR panel was performed in 12 cases, with 3 positive results (Norovirus, Sapovirus, E. coli). Clinically,there were 10 cases of infectious colitis (17\%), 3 which occurred in transplant patients. Other less common causes of active colitis included graft vs host disease (GVHD), diverticulosis, functional diarrhea, autoimmune disease, and ischemia. Unknown causes comprised $13 \%$ of cases with 5 screening colonoscopies performed in asymptomatic patients.

Conclusions: Partially treated/early IBD was the most common cause of "mild colitis", which includes both CD and UC in our subset of cases. Immune modulators should be considered in the differential for mild colitis. Unique infectious etiologies can be detected using PCR based microbiologic panels. Previously unreported causes of mild colitis include GVHD and pancreatic insufficiency.

719 HER2 Expression Is Predominantly Negative in GEJ and Gastric Adenocarcinoma with Signet Ring Cell Differentiation; Study of 346 Cases Heewon Kwak, Tze S Khor, Lindsay Alpert, Daniel Catenacci, Shu-Yuan Xiao, John Hart, Namrata Setia, Priyanthi Kumarasinghe. University of Chicago, Chicago, IL; University of Western Australia, Crawley, WA, Australia.

Background: Currently there are no CAP/ASCP/ASCO-approved guidelines for HER2 testing and scoring of GEJ and gastric cancers. The draft guidelines from 2015 have raised the issue of universal testing of all gastric and esophageal adenocarcinomas. A low prevalence (1-7\%) of HER2 expression has been reported in diffuse-type gastric carcinoma. We investigated the relationship of HER2 status and clinicopathologic features of GEJ and gastric adenocarcinomas with particular emphasis on histologic type, including signet-ring cell differentiation.

Design: GEJ and gastric adenocarcinomas from 05/2007-07/2016 were identified from 2 institutions. HER2 immunohistochemistry (IHC) and HER2 S/FISH amplification were assessed using CAP/ASCO guidelines. Select cases used mass spectrometry (OncoPlex) or next generation sequencing (NGS, FoundationOne $\mathbb{}$ ) to determine HER2 protein quantitation or amplification, respectively. Equivocal HER2 IHC was reflexed to S/ FISH, mass spectrometry, or NGS. Results were compared with age, sex, tumor size, histologic type [Lauren classification and presence or absence of signet ring cells (signet ring cell differentiation)], histologic grade, tumor location, and $\mathrm{T}, \mathrm{N}$, and $\mathrm{M}$ stage, when available. Univariate analysis was performed to determine whether the above variables were predictive of HER2 overexpression/amplification (HER2 positive). 
Results: Of the 346 cases identified, 290 were biopsies and 56 were excisions. Men comprised $74 \%$. The mean age was 63 years (range 16-88 years). Histologic types identified included: intestinal (66\%), diffuse $(25 \%)$ and mixed $(9 \%)$. Overall, 61/346 $(17.6 \%)$ of cases were HER 2 positive. Intestinal, diffuse, and mixed types comprised $89 \%, 11 \%$, and $0 \%$ of HER 2 positive cases, respectively. Only $1 / 46$ cases with signet ring cell differentiation was HER2 positive (IHC). Intestinal type and moderate grade were predictive of HER2 positivity [OR $5.08,95 \%$ CI 1.94-13.35, $\mathrm{p}<0.01$; OR 2.51 , $95 \%$ CI 1.29-4.91, $\mathrm{p}<0.01$, respectively]. Signet ring cell differentiation and poor grade were predictive for the absence of HER2 (OR 11.02, 95\% CI 1.49-81.56, $\mathrm{p}=0.02$; OR $2.33,95 \%$ CI $1.20-4.57, \mathrm{p}=0.01$, respectively).

Conclusions: Expression/amplification of HER2 is unlikely in the presence of signetring cell differentiation. Intestinal type and moderate grade were predictive of HER2 positivity, while poor grade was a negative predictor. Based on our findings, we recommend incorporation of signet ring cell differentiation in the upcoming guidelines for HER2 testing in GEJ and gastric cancers.

720 Site-Specific Molecular Alterations in Colorectal Carcinoma: KRAS Mutations Are More Frequently Identified in Cecal Adenocarcinoma Compared to Other Location Subsites

Michael Landau, Reetesh Pai. University of Pittsburgh Medical Center, Pittsburgh, PA. Background: Recent literature indicates that colorectal carcinoma (CRC) can be subdivided into clinically relevant subtypes based on mutations in $B R A F$ and $K R A S$ and DNA mismatch repair (MMR) protein abnormalities. However, CRC subsite specific molecular differences have not been fully elucidated.

Design: 413 consecutively resected CRCs over a 2-year period were analyzed for mutations in $B R A F$ and $K R A S$ and for MMR protein abnormalities by immunohistochemistry. The frequencies of molecular and histologic features as well as molecular subclassification (KRAS wild-type/BRAF wild-type/MMR proficient $K R A S$-mutated/MMR proficient, $B R A F$-mutated/MMR proficient, and MMR protein deficient regardless of $K R A S$ and $B R A F$ status) were examined along bowel subsites. Results: The table details the differences in molecular subtypes stratified by tumor subsites. Cecal tumors were more often categorized as the KRAS-mutated/MMR proficient molecular subtype ( $43 \%$ ) compared to other tumor subsites within the right colon $(24 \%)(\mathrm{p}=0.02)$ and compared to tumors of the left colon/rectum $(31 \%)(\mathrm{p}=0.04)$ $B R A F$-mutated/MMR proficient status was more often seen in cecal tumors $(5 \%)$ and other tumor subsites in the right colon (11\%) compared to tumors of the left colon/rectum $(1 \%)(\mathrm{p}=0.01)$. Tumors of the left colon/rectum more frequently demonstrated concurrent $K R A S$ wild-type, $B R A F$ wild-type, and MMR proficient status (62\%) compared to cecal tumors $(28 \%)$ and other tumor subsites of the right colon $(37 \%)(\mathrm{p}<0.001)$

\begin{tabular}{|l|c|c|c|c|c|c|}
\hline $\begin{array}{l}\text { Molecular } \\
\text { Subtype }\end{array}$ & $\begin{array}{c}\text { Cecum } \\
(\%)\end{array}$ & $\begin{array}{c}\text { Right } \\
\text { Colon } \\
\text { (All Other } \\
\text { Subsites) } \\
(\%)\end{array}$ & $\begin{array}{c}\text { Left } \\
\text { Colon/ } \\
\text { Rectum } \\
(\%)\end{array}$ & $\begin{array}{c}\text { p-value } \\
\text { (All } \\
\text { Categories) }\end{array}$ & $\begin{array}{c}\text { p-value } \\
\text { (Cecum } \\
\text { vs. Other } \\
\text { Right Colon } \\
\text { Subsites) }\end{array}$ & $\begin{array}{c}\text { p-value } \\
\text { (Cecum vs. } \\
\text { Left Colon/ } \\
\text { Rectum) }\end{array}$ \\
\hline $\begin{array}{l}\text { Number } \\
\text { Tested }\end{array}$ & 83 & 95 & 235 & NA & NA & NA \\
\hline $\begin{array}{l}\text { KRAS } \\
\text { mutated, } \\
\begin{array}{l}\text { MMR } \\
\text { proficient }\end{array}\end{array}$ & $36(43)$ & $23(24)$ & $73(31)$ & 0.02 & 0.007 & 0.04 \\
\hline $\begin{array}{l}\text { BRAF } \\
\text { mutated, } \\
\text { MMR } \\
\text { proficient }\end{array}$ & $4(5)$ & $10(11)$ & $3(1)$ & 0.01 & 0.2 & 0.06 \\
\hline $\begin{array}{l}\text { MMR } \\
\text { deficient, } \\
\text { regardless } \\
\text { of KRAS/ } \\
\text { BRAF status }\end{array}$ & $20(24)$ & $27(28)$ & $14(6)$ & $<0.001$ & 0.5 & $<0.001$ \\
\hline
\end{tabular}

Conclusions: Our findings indicate there are significant site-specific differences in molecular subtypes in CRC. In particular, the findings challenge the dichotomous division of CRC into right versus left colon/rectum and suggest that cecal tumors represent a potentially unique subsite of CRC characterized by a high frequency of KRAS mutations compared to other colorectal subsites.

721 Squamoid Morule in the Pseudoinvasive Foci of Colonic Adenomatous Polyp Morphologically Mimics Invasive Carcinoma

Hee Eun Lee, Vishal S Chandan, Chung-Ta Lee, Tsung-Teh Wu. Mayo Clinic, Rochester, MN; National Cheng Kung University Hospital, Tainan, Taiwan.

Background: Colorectal adenoma can show focal squamous differentiation or squamoid morule (SqM). SqMs are often presented as solid nests or pseudocribriforms, which may cause diagnostic challenge when grading dysplasia. We describe detailed histologic findings of SqM in the pseudoinvasive foci of colorectal adenomatous polyp mimicking invasive carcinoma.

Design: Five colonic adenomatous polyps with $\mathrm{SqM}$ in the pseudoinvasive foci ( 3 with polypectomy and 2 colectomy) were collected from our consultation files. Histologic review and immunostain for cytokeratin (CK) 5/6, p63, synaptophysin and chromogranin were performed on the cases with SqM. Forty-seven consecutive colorectal adenomatous polyps with pseudoinvasion, none of which showed SqM by $\mathrm{H} \& \mathrm{E}$ slides review, were included as a control group, and clinicopathologic features were compared with cases with SqM.

Results: Cases with $\mathrm{SqM}$ more frequently occurred in younger (mean $\pm \mathrm{SD}$, years $=$ $61.4 \pm 11.9$ vs $65.8 \pm 11.7 ; \mathrm{p}=0.047)$ and male patients $(5 / 5$ vs $30 / 47 ; \mathrm{p}=0.101)$ and located in the right colon $(3 / 5$ vs $8 / 47 ; \mathrm{p}=0.027)$ than those without SqM. Pathologic diagnosis of the polyps included tubular/tubulovillous adenoma (TA/TVA) with low-grade (LG) dysplasia (with SqM, n=3; vs without SqM, n=29), TA/TVA with high-grade (HG) dysplasia $(\mathrm{n}=2$ vs $\mathrm{n}=15)$, and sessile serrated adenoma (none $v s \mathrm{n}=3)$, of which frequency was not different between two groups. Histologic examination demonstrated that $\mathrm{SqMs}$ replaced glandular epithelium often forming a pseudocribriform or formed a solid nest sitting in the stroma of pseudoinvasive foci. SqMs were classified into two distinct histologic patterns: 1) "immature squamous pattern" mimicking immature squamous metaplasia and forming a well-formed morular structure $(\mathrm{n}=3)$ and 2 ) "mature squamous pattern" resembling mature squamous metaplasia $(n=2)$. Both patterns showed CK5/6 positivity, which tended to be more diffuse and stronger in the mature squamous pattern, and p63 negativity. Synaptophysin and chromogranin were found to be focal positive in both patterns. Both polyps with mature squamous pattern showed TVA with focal HG dysplasia as compared with TA with LG dysplasia seen in all three with immature squamous pattern.

Conclusions: Squamoid morules in colonic adenomatous polyps can mimic invasive carcinoma when present in the pseudoinvasive foci. Pathologists should be aware of its presence and morphology in the colorectal adenoma to prevent over diagnosis of invasive carcinoma.

722 Association of Micropapillary Architecture and High Tumor Budding with Prognosis in Patients with Stage III Colon Cancer from a FOLFOX-Based Adjuvant Chemotherapy Trial: NCCTG N0147 (Alliance) Hee Eun Lee, Qian Shi, Nathan R Foster, Emily Chan, Sharlene Gill, Morton Kahlenberg, Suresh Nair, Anthony Shields, Richard M Goldberg, Steven R Alberts, Frank A Sinicrope, Thomas Smyrk. Mayo Clinic, Rochester, MN; The Ohio State University Comprehensive Cancer Center, Columbus, OH; Vanderbilt University School of Medicine, Nashville, TN; BCCA-Vancouver Cancer Centre, Vancouver, Canada; Surgical Oncology Associates of South Texas, San Antonio, TX; Lehigh Valley Health Network, Allentown, PA; Wayne State University, Karmanos Cancer Institute, Detroit, MI.

Background: We hypothesized that high tumor budding and micropapillary architecture would be individually associated with poor disease-free (DFS) and overall survival (OS) and that the presence of both features would have an additive effect in colon carcinoma patients.

Design: Histologic tumor sections from Stage III colon cancer patients $(\mathrm{N}=1704)$ treated in a randomized trial of adjuvant FOLFOX+/-cetuximab were analyzed for micropapillary architecture (considered present $[>0 \%]$ or absent) and tumor budding (200x field; $\geq 10$ buds $=$ high budding). Chi-squared or Wilcoxon Rank sum tests were used to assess the associations between clinicopathologic variables, as well as KRAS, $B R A F^{\mathrm{V} 600 \mathrm{E}}$, and mismatch repair status (MMR). Prognosis was evaluated using adjusted multivariable Cox models for DFS and OS.

Results: The median tumor bud count was 5 and high tumor budding was detected in 506 tumors (29.7\%). Micropapillary architecture was identified in 380 tumors $(22.3 \%)$ in amounts ranging from 1 to $90 \%$ of tumor area. Both features of interest were seen in 199 patients $(11.7 \%)$; $1016(59.6 \%)$ had neither. Presence of micropapillary or high tumor budding were each associated with T3/4 vs $1 / 2$, N2 vs N1, high vs low grade, and proficient MMR vs deficient MMR. High tumor budding was associated with proximal site, mutations in $K R A S$ or $B R A F$, and low tumor infiltrating lymphocytes. High tumor budding was associated with shorter DFS (HR 1.29, $\mathrm{p}=0.009)$ and shorter OS (HR 1.37, $\mathrm{p}=0.002$ ). Micropapillary architecture was not significantly associated with DFS or OS. DFS of patients with both features did not differ significantly from those with only one feature, but patients with both features had shorter OS compared to those with only positive micropapillary architecture (HR 1.46, $\mathrm{p}=0.044$ ).

Conclusions: High tumor budding and micropapillary architecture are both associated with markers of tumor aggressiveness. Tumor budding was a significant and independent predictor of poor disease outcomes. Support: U10CA180820, U10CA180821, U10CA180835, U10CA180882, and U10CA180888. ClinicalTrials.gov Identifier: NCT00079274.

723 Crypt Apoptosis Contributes to Mucosal Flattening in Celiac Disease Patients When Exposed to Dietary Gluten

Michael Lee, Alina Iuga, Hui-Min Yang, Jude Fleming, Peter HR Green, Benjamin Lebwohl, Stephen M Lagana. Columbia University, New York, NY.

Background: Celiac disease is characterized histologically by inflammation and villous atrophy. The mechanism of villous atrophy is thought to be a disruption in the balance between epithelial proliferation and death. Epithelial cells in intestinal mucosa normally proliferate in crypts and migrate towards the lumen where they undergo apoptosis. Apoptotic bodies in crypts are usually abnormal and are associated with certain disease states. The presence of crypt apoptosis in celiac disease has not been thoroughly examined (data exists regarding epithelial apoptosis markers, but a histologic assessment of crypt apoptotic body count (ABC) has not been published). We quantified the ABC in duodenal biopsies from celiac patients before and after initiation of a gluten free diet (GFD) and compared them to normal controls (patients with heartburn).

Design: We examined twenty-five duodenal biopsies from patients with celiac disease at diagnosis and following initiation of GFD and determined the maximum ABC in 10 consecutive crypts. Fourteen duodenal biopsies from patients with heartburn served as normal controls.

Results: Participants had a mean age of 44 years at diagnosis and $36 \%$ were male. Mean duration between paired biopsies was $2.9(0.5-8.5)$ years. The mean tissue transglutaminase was 4.8 times the upper limit of normal at diagnostic biopsy and 0.7 times the upper limit of normal at follow-up biopsy. Mean maximum ABC in active celiac disease (ACD) was 5.44 and decreased to 2.60 after initiation of GFD $(p=<.0001)$. 
The mean maximum $\mathrm{ABC}$ in controls was 1.79 , which was less than both $\mathrm{ACD}$ and GFD ( $p=<.0001$ and $p=.019$ respectively). Completely flat lesions (mean: 6.44$)$ also showed a higher $\mathrm{ABC}$ compared to non-flat lesions (mean: 4.87); $p=.04$.

Conclusions: Crypt $\mathrm{ABC}$ is markedly elevated in $\mathrm{ACD}$ and decreases significantly with GFD, however it does not achieve normalcy. In ACD patients, flat mucosa (Marsh $3 C$ ) is associated with a higher $\mathrm{ABC}$ than all other Marsh lesions. It is likely that crypt apoptosis is important in the mucosal flattening of celiac disease.

$724 \quad$ Risk Factors of Lymph Node Metastasis in T1 Colorecta Cancer: Tumor Budding Is a Reliable Pathologic Indicator Than Depth of Submucosal Invasion

So Jeong Lee, Do Youn Park, Kyung Un Choi, Gi Young Huh, Chang Hun Lee, Ahrong Kim, Young Keum Kim, Chung Su Hwang. Pusan National University Hospital, Busan, Seo-Gu, Republic of Korea.

Background: With development of endoscopic treatment modalities for T1 colorectal cancer (CRC), it is imperative to accurate determine risk of lymph node (LN) metastasis in T1 colorectal cancer (CRC). We investigated risk factors of lymph node metastasis in T1 CRC to develop safe guideline for endoscopic resection of T1 CRC.

Design: Study cohorts included 133 cases of T1 CRC from January 2010 to June 2016. We reviewed clinicopathological features of all cases, including gross type, histological differentiation, lymphatic invasion, vascular invasion, and tumor budding. We also measured submucosal invasion depth (SID) by macroscopic type and muscularis mucosa status.

Results: Among133 cases of T1 CRC, 16 cases showed LN metastasis. Interestingly, there are no statistical difference of depth of submucosal invasion between $\mathrm{LN}(+)$ (2246.88 \pm 1097.606$)$ and $\mathrm{LN}(-)(3558.82 \pm 8189.056)$ groups (SID $\mu \mathrm{m} \pm$ standard deviation, $\mathrm{p}=0.525$ ). Furthermore, there was no association between lymphovascular tumor emboli and LN metastasis. Presence of tumor budding and higher number of tumor budding are reliable indicator for $\mathrm{LN}$ metastasis in T1 CRC $(\mathrm{p}<0.005)$. Presence of adenoma component is negative indicator for $\mathrm{LN}$ metastasis $(\mathrm{p}=0.036)$.

Conclusions: We found tumor budding is a reliable indicator than depth of submucosal invasion and recommend careful identification of tumor budding in routine surgical practice of T1 CRC.

725

Loss of Cellular Histone Modifications in Pancreatic Adenocarcinoma

Hongjie Li, Aaron E Harper, Danqi Chen, Chunyuan Jin, Steve Xie. Downstate Medical Center, Brooklyn, NY; New York University, Tuxedo Park, NY; Kings County Hospital, Brooklyn, NY.

Background: A recent comprehensive integrated genomic analysis of 456 pancreatic ductal carcinomas identified 32 significant mutated genes that aggregate into 10 molecular mutational mechanisms. Histone modification is determined as one of these important mechanisms and the mutated genes identified in $24 \%$ of the cases include KDM6A, SETD2 and ASCOM complex members MLL2 and MLL3. KDM6A is a histone demethylase that specifically demethylates "Lys-27" of histone H3 (H3K27me3), which is associated with repression of gene activity. ASCOM possesses histone methylation activity that specifically methylates "Lys-4" of histone H3 (H3K4Me3), which is associated with activation of gene activity. These mutated genes therefore might lead to global changes in H3K27me3 and H3K4me3, which could be used as diagnostic/prognostic biomarkers of pancreatic ductal carcinomas.

Design: Here we investigated cellular expression levels of H3K27me3 and H3K4Me3 in pancreatic adenocarcinomas as well as nonneoplastic pancreatic tissues, aiming to explore the diagnostic/prognostic values of epigenetic markers in pancreatic carcinogenesis.

Results: Twelve pancreatic adenocarcinoma samples were examined. Immunohistochemistry was performed on formalin-fixed, paraffin-embedded tissue blocks by using antibodies against $\mathrm{H} 3 \mathrm{~K} 27 \mathrm{me} 3$ and $\mathrm{H} 3 \mathrm{~K} 4 \mathrm{me}$. The staining intensity of cancer tissues was quantified by the NIH ImageJ software and compared to the staining intensity of peritumoral normal ducts. The cellular levels of both H3K27me3 and $\mathrm{H} 3 \mathrm{~K} 4 \mathrm{me} 3$ were dramatically downregulated in all pancreatic adenocarcinoma samples we tested compared with peritumoral normal ducts. The difference in the levels of H3K27me3 and H3K4me3 between normal pancreatic ducts and pancreatic adenocarcinomas was statistically significant $(p<0.05)$.

Conclusions: H3K27me3 and H3K4me3 are associated with gene inactivation and activation, respectively. Thus, aberration in these modifications might be involved in pancreatic carcinogenesis through changing gene expression. In this preliminary study, we found significantly decreased levels of H4K27me3 and H3K4me3 in all twelve samples, suggesting that the loss of these modifications might be used as biomarkers for pancreatic adenocarcinoma. The correlation between the cellular levels of these histone modifications and clinicopathologic parameters and clinical outcomes are currently under investigation in a larger scale study.
Does Serrated Polyp Unclassified Exist? A Nationwide Study and Survey

Jingmei Lin, Dale C Snover, Robert D Odze, Xiuli Liu, Hwajeong Lee, Maria Westerhoff. Suntrea TG Hammer, ILKe Nalbantoglu, Oscar W Cummings, Hanlin L Wang, Barbara $J$ McKenna, Scott R Owen, Jonathan McHugh, Romil Saxena, Jiaqi Shi, Eun-Young $K$ Choi, Ashwini K Esnakula, Jesse L Kresak, John R Goldblum, Henry D Appelman, Douglas K Rex. Indiana University, Indianapolis, IN; Fairview Southdale Hospital, Edina, MN; Brigham and Womens Hospital, Boston, MA; University of Florida, Gainesville, FL; Albany Medical College, Albany, NY; University of Washington, Seattle, WA; University of Texas Southwester, Dallas, TX; Washington University, Saint Louis, MO; University of California, Los Angeles, CA; University of Michigan, Ann Arbor, MI; Cleveland Clinic, Cleveland, OH.

Background: Colorectal serrated polyps consist mainly of hyperplastic polyp, sessile serrated adenoma/polyp (SSA/P) and traditional serrated adenoma. Morphological differentiation of serrated polyps is usually not difficult, although it may be challenging when ambiguous features exist. In 2012, a terminology of "serrated polyp unclassified" (SPU) was coined for serrated polyps that might be difficult to classify. Since then, there is a paucity of data related to the acceptance of SPU in pathology community.

Design: We conducted a nationwide study and survey about SPU. Totally, 20 gastrointestinal pathologists from 11 academic centers were invited to review 16 serrated polyps with equivocal features that were selected from 513 consecutive rectosigmoid serrated polyps.

Results: Not a single polyp gained a complete agreement from all 20 pathologists. An alternative conveyed consensus was made if any diagnostic category had been voted by more than $60 \%$ of the participating pathologists. Based on this criterion, 7 serrated polyps has reached a classification (2 hyperplastic polyps and $5 \mathrm{SSA} / \mathrm{Ps}$ ) and the other 9 fell into SPU with a frequency rate of $1.8 \%$. Additionally, the survey indicated that $61 \%$ of the gastrointestinal pathologists were aware of SPU, although majority $(83 \%)$ never used it.

Conclusions: To our knowledge, this is the first study to investigate the nationwide recognition of SPU and to provide adequate histological illustrations. Although SPU hasn't been well accepted by the pathologist community, proper pathologic examples and/or definition may help pathologists to recognize. Lack of appropriate clinical management hinders the acceptance of SPU that requires more input from clinicopathological follow-up.

727

Characterization of Intestinal Pathology in Adults with Cystic

Fibrosis

Leo Lin, Grace Y Lin, Paul Quinton, Douglas Conrad, Mojgan Hosseini. UCSD, La Jolla, CA.

Background: Cystic fibrosis is the most common autosomal recessive disease among Caucasians. It affects 1 in 2,500 Caucasian births with a carrier frequency of $1 / 25$. Due to advances in medical care, the mean age of survival has dramatically increased from the early twenties in the 1980's to almost forty today. Recent studies have not demonstrated a higher risk of lung malignancies with increased lifespan; however, they have shown a dramatically higher risk of GI malignancies (increased relative risk of small bowel and colon cancer are 24.8 and 7.4 respectively) which occur at a much younger age ( 32 years vs 58 years in non-Hispanic whites). There is often a delay in making the diagnosis, as routine colonoscopies are done at age 50. The histopathology of the GI tract in adult CF patients has not yet been well described.

Design: We conducted a retrospective search of our institutional archives (2011-2016) and identified 6 adult $\mathrm{CF}$ patients with 8 cases. We only included biopsies taken for surveillance due complaints such as abdominal pain (excluding ostomy take downs). This left us with 6 cases from 5 CF patients who were 25 to 41 years old when the biopsies were taken. $\mathrm{H} \& \mathrm{E}$ slides were reviewed.

Results: Inspissated eosinophilic material was observed in the crypts of the ileum and colon of all adult CF patients. Maximum abundance was noted in the duodenum and right colon, decreased in the left colon, and was scant or absent in the sigmoid colon and rectum. The number of intraepithelial neutrophils, eosinophils and lamina propria eosinophils paralleled the rise and fall of the luminal inspissated material. All ileal biopsies had eosinophilic crypt abscesses (3 cases from 3 patients).

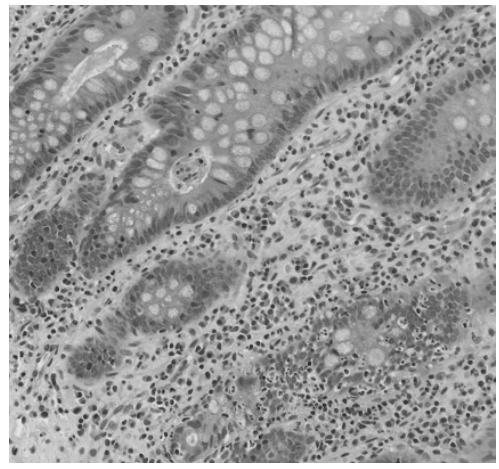

Conclusions: All five adult CF patients had eosinophilic inspissated material within the small and large intestinal crypts. The rise and fall of this luminal eosinophilic material was associated with a similar pattern of rise in the number of intraepithelial and lamina propria eosinophils and intraepithelial neutrophils. It will be important to determine if this luminal eosinophilic material is chemoatractant to neutrophils or eosinophils and if this inflammation is contributing to the dramatically higher rates of GI malignancies in CF patients. 
Cheng Liu, Neal Walker, Mark Bettington, Christophe Rosty, Barbara Leggett, Vicki Whitehall. QIMR Berghofer Medical Research Institute, Brisbane, Australia; Envoi Specialist Pathologists, Brisbane, Australia; Royal Brisbane and Women's Hospital, Brisbane, Australia; University of Queensland, Brisbane, Australia.

Background: Dysplasia occurring in sessile serrated adenoma (SSA) is uncommon and it heralds rapid progression to invasive carcinoma. The WHO definition for SSA with dysplasia (SSAD) divides it into conventional and serrated types, but it has become apparent the morphology of SSADs falls on a spectrum and some variants are difficult to classify using current criteria. We undertook a comprehensive review of all SSADs diagnosed over a four-year period to fully characterize these variants.

Design: SSADs diagnosed at a specialist gastrointestinal pathology practice between 2013 and 2016 were retrospectively identified through database search. A total of 254 SSADs were reviewed and classified based on architectural and cytologic features. When more than one type of dysplasia was present in an SSAD, they were classified separately. MLH1 immunohistochemistry was performed in all cases.

Results: We identified several distinct variants of SSAD. Most notable was the "minimal deviation" variant, present in 12 of 254 cases, which demonstrated only subtle architectural abnormalities compared with the background non-dysplastic SSA. These included gland crowding, gland dilatation, horizontal growth of glands, and excessive luminal serrations or a smooth luminal profile. There was no cytologic atypia. A dysplastic interpretation was supported by MLH1 loss and the presence of overt dysplasia elsewhere in the same biopsy. Other variants displayed similarities to traditional serrated adenoma (TSA) or conventional adenoma, and thus had the potential for both under- and overdiagnosis.

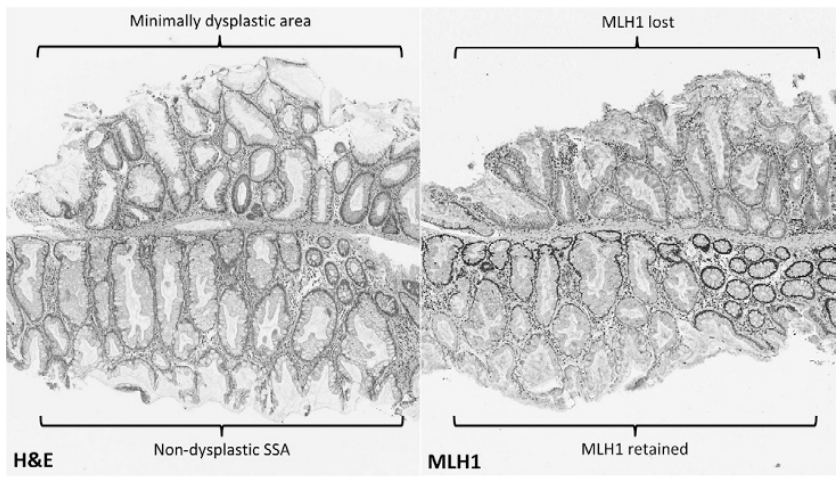

Conclusions: SSADs exhibit a wide range of morphologic appearances. We have devised a SSAD classification system which better reflects the observed features than the current WHO definition. The "minimal deviation" variant displays subtle changes which are easily overlooked in cases without overt dysplasia elsewhere, and we advocate a low threshold for performing MLH1 immunohistochemistry whenever an SSA contains unusual architectural patterns. When eosinophilic cells are present, TSA should be excluded prior to diagnosing SSAD.

729

Gene Expression Profiling in Collagenous Colitis: Toward a Better Understanding of the Disease

Qingqing Liu, Huai-Bin Mabel Ko, Hongfa Zhu, Alexandros D Polydorides, Noam Harpaz. Ichan School of Medicine at Mount Sinai, New York, NY.

Background: Collagenous colitis (CC) is a common but enigmatic diarrheal disorder that is thought to result from dysregulated mucosal immune responses to unknown luminal agents in genetically susceptible individuals. Its association with immune dysregulation is suggested epidemiologically by its close associations with celiac disease and a variety of autoimmune disorders. We aimed to probe its pathogenesis by means of gene expression profile analysis with a focus on inflammatory and immunological pathways.

Design: mRNA was isolated from colonic biopsies of 13 histologically confirmed patients with $\mathrm{CC}$ and analyzed by the NanoString nCounter gene

expression assay. The analysis included a comparison of mucosa with and without abnormal subepithelial collagen ("involved" and "uninvolved") $(\mathrm{N}=5)$, pooled biopsies of involved mucosa of additional patients $(\mathrm{N}=8)$ and pooled biopsies from normal controls $(\mathrm{N}=8)$. Assays targeted 778 human genes, 594 differentially expressed in immunological disorders and 184 expressed in inflammatory conditions. The raw expression data were normalized using nSolver Analysis Software 3.0 and a dataset of gene expression ratios for CC vs controls was generated.

Results: Our results showed increased expression of matrix-matalloproteinases (MMP$3,7.84 \mathrm{X}$ and MMP-9, 3.65X) in all cases of CC, confirming the results of a prior study. Interferon gamma (INFG) was markedly upregulated (31X) in all cases of CC and was accompanied by corresponding upregulation of its downstream genes including STAT1 (3.48X) of the JAK-STAT pathway, multiple members of the T-lymphocyte chemoattractant chemokine (C-X-C motif) ligand family (CXCLs, 5.07-13.39X) and their receptors (CXCRs, 2.79-5.71X). Additionally, we noted enrichment of mRNA expression for HLA-DQA1(1.2X) and HLA-DQB1(1.98X) in our CC population, a feature shared with celiac disease. Each of these findings was observed in both involved and uninvolved mucosa of CC cases.

Conclusions: The colonic mucosa in CC is characterized by enhanced expression of a limited repertoire of immunological and inflammatory genes. The nature of the corresponding pathways may help guide further investigations into its etiology. The fact that these alterations are expressed in histologically uninvolved as well as involved mucosa suggests that the underlying pathology is more globally distributed than its characteristically patchy histology.

\section{Diagnostic Value of Stage-Specific Expression of SMAD4 and} TP53 in Barrett's-Associated Neoplasia

Qingqing Liu, Huai-Bin Mabel Ko, Hongfa Zhu, Alexandros D Polydorides, Noam Harpaz. Ichan School of Medicine at Mount Sinai, New York, NY.

Background: Accurate classification of biopsies with Barrett's-associated highgrade dysplasia (HGD) and invasive adenocarcinoma carries important therapeutic implications but can be challenging. Histological criteria such as solid or cribriform growth pattern, intratubular necrosis and ulceration have proven useful, albeit imperfect, in predicting the presence of invasion in resection specimens. A recent cancer genome sequencing study of Barrett's metaplasia, dysplasia and adenocarcinoma reported that inactivating mutations in the p53 and SMAD4 genes occurred uniquely in HGD and/ or carcinoma in a stage-specific manner, i.e., p53 in $70 \%$ of HGD and carcinoma and SMAD4 in $10 \%$ of carcinoma only. To our knowledge these findings have not been exploited diagnostically by immunohistochemical means.

Design: TP53 and SMAD4 immunostaining were performed on a series of 20 specimens, 10 biopsies with HGD and 10 biopsies or resection specimens with intramucosal adenocarcinoma (IMC). All diagnoses were confirmed by a group of 5 GI pathologists at a divisional consensus conference and based on clinical follow-up. Wild-type TP53 expression was defined as heterogeneous nuclear expression of $2+$ or less and mutanttype expression as absent expression or homogeneous overexpression of $2+$ or greater. Wild-type SMAD4 expression was defined by nuclear expression comparable to that in the surrounding normal tissue and mutant-type expression by absent expression. Results: Mutant-type TP53 expression occurred in 8 of 10 cases of $\operatorname{HGD}(80 \%)$ and in 10 of 10 cases of IMC $(100 \%)$. In contrast, mutant-type SMAD4 expression occurred in 0 of 10 cases of HGD and in 3 of 10 cases of IMC $(30 \%)(p=0.031$, chi square method). Conclusions: Our results are compatible with the findings reported by cancer genome sequencing. Stage-specific immunostaining for SMAD4, if confirmed in larger studies, may afford a valuable adjunct to conventional histological criteria in achieving accurate diagnoses of invasive Barrett's adenocarcinoma.

\section{Incidence and Prognosis of Extramural Venous Invasion in} Small Intestine Neuroendocrine Tumors

Qingqing Liu, Huai-Bin Mabel Ko, Hongfa Zhu, Noam Harpaz, Alexandros D Polydorides. Ichan School of Medicine at Mount Sinai, New York, NY.

Background: Extramural venous invasion (EVI) is a well-established independent prognostic factor in colorectal carcinoma and has been linked to distant hematogenous spread (e.g., to the liver), thus influencing the clinical decision to administer adjuvant chemotherapy. However, the prognostic significance of EVI in small bowel neuroendocrine tumors (NET) has not been extensively studied and it is not routinely assessed or reported.

Design: We retrospectively reviewed resected small bowel NETs at our institution over a 6 year period (2010-2016). H\&E slides were independently scored by two pathologists for the presence of EVI, defined as the unequivocal presence of NET deposits within the lumen of large subserosal veins, the latter being in close proximity to large arteries. Elastica van Giesonstaining (EVG) was selectively used as an adjunct method in equivocal cases. Information on clinicopathologic features, including the presence of liver metastases, was obtained from pathology reports and medical records. Fisher's exact test was used to determine statistical significance (defined as $P<0.05$ ). Results: Seventy-seven patients with primary small bowel NET (41 women and 36 men; median age 62 years, range: 31-84 years; 3 duodenal, 31 ileal, and 43 small bowel, not otherwise specified) were included in the study. EVI was identified in 42 cases $(54.5 \%), 31$ of which $(73.8 \%)$ had liver metastases either at the time of resection or subsequently developed. This incidence was significantly higher than that observed in patients without evidence of EVI $(\mathrm{N}=35)$, only 6 of whom $(17.1 \%)$ had liver metastases $(P<0.0001)$. Presence or absence of EVI did not correlate with pathologic tumor stage (pT) or lymph node status $(\mathrm{pN})(P>0.05)$.

Conclusions: Our data demonstrate that EVI is commonly present in small bowel NETs and strongly correlates with the presence of liver metastases. Therefore, its evaluation is critical during the pathologic examination of resection specimens with these tumors and should be assessed in combination with adjuvant techniques such as EVG, if necessary. Moreover, EVI status may need to be included in the pathology reporting guidelines for small bowel NETs.

732 Expression of PD-L1 in Colorectal Carcinoma (CRC) Primarily Occurs in Stromal/immune Cells at Tumor-Stroma Interface (TSI), and Is Associated with High Tumor-Infiltrating Lymphocytes (TILs) Irrespective of the Microsatellite Instability (MSI) Status or the Molecular Mechanism of MSI

Sandy Liu, Jaclyn Hechtman, Neil Segal, Joshua Smith, Deepthi Rao, Arnold Markowitz, Martin Weiser, Efsevia Vakiani, David S Klimstra, Zsofia Stadler, Jinru Shia. Memorial Sloan Kettering, NY, NY.

Background: Expression of PD-L1 has been observed in a subset of MSI-H CRCs. It remains to be determined whether PD-L1 expression is driven by MSI-H or TILs. Design: We studied PD-L1 and PD-1 expression via immunohistochemistry (IHC) on whole sections in the following 5 groups of cases $(\mathrm{n}=78)$ : 1) 16 MSI-H CRCs from 16 Lynch-syndrome patients with mismatch repair (MMR)-gene-germline mutation; 2) 21 sporadic MSI-H CRCs with MLH1-promoter methylation; 3) 13 MSI-H CRCs without germline-mutation or MLH1-methylation; 4) 13 microsatellite-stable (MSS) CRCs with unusually high TILs (MSS-hTIL); and 5) 15 MSS CRCs with low TILs 
(MSS-low-TIL). The staining intensity for both markers was scored 0-3+. The extent of PD-L1-staining was scored as \% of TSI area showing positive staining, and the extent of PD-1-stained-TILs was semi-quantitatively graded (0-3).

Results: The mean "TILs/10HPFs" ranged 27-57 in the 3 MSI-H groups, and was 40 in MSS-hTIL and 3 in MSS-low-TIL groups $(\mathrm{p}=0.02)$. IHC expression of PD-L1 was primarily present in stromal lymphocytes/immune cells at TSI; less commonly, it was also seen within tumor cells. The intensity and expression of PD-L1 at TSI were not different across the 3 MSI-H groups and the MSS-hTIL group $(\mathrm{p}=0.33)$. However, both were significantly higher in all 4 groups (individually or collectively) when compared to the MSS-low-TIL group $(\mathrm{p}<0.001)$. Similarly, both the intensity and extent of PD1 -staining in TILs did not differ across the MSI-H and MSS-hTIL groups $(\mathrm{p}=0.15)$, but in the latter 4 groups, the PD-1 intensity was significantly higher when compared to MSS-low-TIL-group $(\mathrm{p}<0.001)$.

Conclusions: Our results reaffirm prior studies that expression of PD-L1 occurs in a subset of MSI-H CRCs. Further, we demonstrated for the first time that PD-L1 expression is a function of high TILs irrespective of the MSI status or the molecular mechanism causing MSI. The common perception that MSS tumors lack PD-L1 expression is likely due to the low incidence of hTILs in these tumors. Our observations bear potential implication in the determination of patient-selection method for anti-PD1 trials for CRC.

\section{Calretinin Positivity in Poorly Differentiated Colorecta} Carcinoma: A Diagnostic Pitfall

Sandy Liu, Deepthi Rao, Jaclyn Hechtman, Efsevia Vakiani, David S Klimstra, Jinru Shia. Memorial Sloan Kettering, NY, NY.

Background: Calretinin, a calcium-binding protein encoded by the CALB2 gene and involved in calcium signaling, is commonly used as an immunohistochemical marke for the diagnosis of mesothelioma. We have recently encountered a poorly differentiated colonic adenocarcinoma showing diffuse positivity for calretinin, resulting in diagnostic confusion. In this study, we systematically evaluated the frequency and pattern of calretinin IHC positivity in colorectal carcinoma (CRC).

Design: The study cases consisted of a consecutive series of $257 \mathrm{CRCs}$ that fulfilled a set of relaxed criteria for Lynch-syndrome screening; 59 (23\%) were mismatch-repair (MMR)-deficient by immunohistochemistry (IHC) (44 MLH1/PMS2-deficient, 9 MSH2/MSH6-deficient, 4 MSH6-deficient, and 2 PMS2-deficient). Tissue arrays were developed. IHC for Calretinin (Ventana, Clone SP65) was performed and evaluated along with various clinoco-pathological characteristics.

Results: In total, positive calretinin staining was observed in 3 of the $257(1 \%)$ CRCs As summarized in Table 1, all 3 positive cases were poorly differentiated, accounting for $14 \%(3 / 21)$ of poorly differentiated CRCs; and all 3 showed MMR deficiency with IHC loss of MLH1/PMS2, constituting 7\% (3/44) MLH1/PMS2-deficient tumors and $5 \%(3 / 59)$ of all MMR-deficient tumors

\begin{tabular}{|l|l|l|l|l|l|l|l|}
\hline $\begin{array}{l}\text { Age/ } \\
\text { Sex }\end{array}$ & Site & Histology & TILs/10HPF & MMR IHC & TNM & $\begin{array}{l}\text { FU } \\
\text { time }\end{array}$ & $\begin{array}{l}\text { FU } \\
\text { status }\end{array}$ \\
\hline $85 /$ F & Rt Colon & $\begin{array}{l}\text { Poor } \\
\text { differentiation } \\
\text { with medullary } \\
\text { features }\end{array}$ & 45 & $\begin{array}{l}\text { MLH1/ } \\
\text { PMS2 loss }\end{array}$ & pT1N0 & $15 \mathrm{mo}$ & NED \\
\hline $82 / \mathrm{F}$ & Rectum & $\begin{array}{l}\text { Poor } \\
\text { differentiation } \\
\text { with medullary } \\
\text { features }\end{array}$ & 244 & $\begin{array}{l}\text { MLH1/ } \\
\text { PMS2 loss }\end{array}$ & pT2N0 & $62 \mathrm{mo}$ & NED \\
\hline $70 / \mathrm{M}$ & Rt Colon & $\begin{array}{l}\text { Poor } \\
\text { differentiation } \\
\text { with medullary } \\
\text { features }\end{array}$ & 54 & $\begin{array}{l}\text { MLH1/ } \\
\text { PMS2 loss }\end{array}$ & pT3N0 & $51 \mathrm{mo}$ & NED \\
\hline
\end{tabular}

Conclusions: Calretinin IHC positivity can be observed in a small but finite proportion of CRCs. Calretinin-positive CRCs share clinico-pathological characteristics with MSI-H tumors. Awareness of this phenomenon can avoid the diagnostic pitfall of misinterpreting poorly differentiated colorectal carcinomas as mesothelioma on the basis of calretinin positivity and prompt additional work-up to allow accurate tumor classification.

\section{$734 \quad$ Next Generation Sequencing Identifies Mutational Distinction Between Primary and Metastatic Colorectal Carcinoma: Potential Therapeutic Implications}

Jean Lopategui, Snehalkumar B Patel, Navid Farahani, Myriam Chevarie-Davis, Mark D Ewalt, Andy Pao, Angela Aguiluz, Robert E Bookstein. Cedars-Sinai Medical Center, Los Angeles, CA.

Background: Colorectal carcinoma (CRC) is a common and aggressive malignancy for which standard chemotherapy is of limited benefit in the metastatic setting. Recently, targeted next generation sequencing (NGS) has emerged as a clinical tool for the identification of actionable gene mutations in order to triage advanced cancer patients to alternative targeted therapies. It is unclear whether primary CRCs or their metastases should be selected for NGS. We explore the mutational distinctions between paired primary and metastatic tumors

Design: A total of 45 formalin-fixed, paraffin-embedded tumors were sequenced from 14 patients, including 15 primary tumors, 10 regional lymph node metastases, 18 distant organ metastases (DM), and 2 recurrences at anastomotic sites. Samples were sequenced using the 50-gene AmpliSeq cancer panel v2, targeting 2855 hotspot mutations.

Results: Ten of 14 patients ( $72 \%$ ) had identical mutational profiles in primary and metastatic sites, two (14\%) had some but not all variants in common among different sites, and two patients (14\%) had sites with entirely unrelated hotspot profiles. In 3 of the 4 nonidentical cases, clinically relevant (actionable) mutations were found in metastases that were not present in primaries. Across all patients, 87 mutations were identified involving 10 genes: TP53, APC, KRAS, NRAS, PIK3CA, FBXW7, BRAF $P T E N, S M A D 4$, and ATM. Seventy-four mutations $(85 \%)$ were concordant between primary tumors and DM.

Conclusions: Our pilot study indicates that most patients have identical mutations in their paired primary and metastatic CRCs, and thus either may be sequenced However, in 3 of $14(21 \%)$ of these patients, additional actionable variants were found in metastases compared to primary tumors. Choice of testing site in such patients may have a significant impact on clinical management, as they may become eligible for investigational targeted therapies in refractory disease. Although extension of this study to a larger patient cohort (ongoing) is necessary before broader conclusions are drawn, a provisional proposal is that genetic profiling of one or more metastases should be considered in the setting of resistance to therapy chosen based on the genetic profile of the primary CRC.

\section{The Autophagy-Associated ATG16L1 Thr300Ala Polymorphism} Affects Outcome of Gastric Adenocarcinoma Patients

Changqing Ma, Ta-Chiang Liu. University of Pittsburgh, Pittsburgh, PA; Washington University, St. Louis, MO.

Background: Autophagy-associated $A T G 16 \mathrm{~L} 1$ gene is known to affect a range of host immune response to pathogens. A non-synonymous single nucleotide polymorphism (SNP) of ATG16L1, Thr300Ala (T300A), is one of the main Crohn disease (CD) susceptibility alleles. Previous studies have shown that mice with Atg16l1 T300A knockin are associated with increased production of inflammatory cytokines albeit reduced pathogen clearance. Given the importance of host immunity to cancer, we hypothesized that host ATG16L1 T300A genotype correlates with clinical outcome in cancer.

Design: Consecutive gastric adenocarcinoma patients who underwent resection between 2008 and 2012 were included. For each patient, genomic DNA was extracted from formalin-fixed paraffin-embedded noncancerous tissue and analyzed by quantitative PCR for $A T G 16 L 1$ T300A genotype. The risk allele for $A T G 16 L 1$ T300A (as per CD susceptibility) is G; the wild-type allele is A. The patients' genotypes were correlated with demographics, various histologic features, and clinical outcome.

Results: A total of 173 patients were genotyped. Among them, $126(73 \%)$ carried the risk allele G, including 44 (25\%) homozygous GG and 82 (47\%) heterozygous GA genotypes. Significantly higher proportions of Caucasian patients carried the G allele(s) compared with those of African American patients $(111 / 135,82 \%$ vs $7 / 20,35 \%, P<$ 0.001). Univariate outcome correlation analysis identified homozygous GG patients had significantly longer overall survival than patients with either GA or AA genotypes (median and range: 40.6 months [4.8-98.2] vs 16.4 [0.5-96.5] vs 21.4 [0.7-106.0], $P=0.019)$. Importantly, in the subset of patients $(75 / 173,43 \%)$ who did not receive additional therapy, patients with homozygous GG genotype still had significantly longer overall survival than the other genotypes by multivariate analysis after adjusting for clinical stage (hazard ratio: $0.279,95 \%$ confidence interval: $0.108-0.719, P=0.008$ ). The ATG16L1 T300A genotype did not correlate with age, gender, histologic grade, depth of invasion, nodal metastasis or recurrence-free survival $(P>0.05$ for all). Conclusions: Gastric cancer patients carrying 2 ATG16L1 T300A risk (G) alleles are associated with improved overall survival, suggesting that $A T G 16 L 1$ may have dichotomous effects in immunity against infection and cancer. In addition, genotyping gastric cancer patients may provide additional insight for management and can be incorporated into standard patient care.

\section{Characterization of Dysplasia in Non-Targeted Colorecta} Biopsies in IBD

Yihong R Ma, Huai-Bin Mabel Ko, Hannah J Sfreddo, Alexandros D Polydorides, Hongfa Zhu, Noam Harpaz. Icahn School of Medicine at Mount Sinai, New York, NY; Washington University, St. Louis, MO.

Background: Colorectal dysplasia complicating IBD is notoriously difficult to detect by standard endoscopy. Surveillance for dysplasia traditionally employs both random biopsy sampling and targeted biopsies of suspicious visible lesions. Recent studies suggest that most dysplasia is visible under optimal conditions, e.g., high-definition optics and/or chromoendoscopy, leading some authorities to question the value of random biopsies altogether. It follows that more information is needed regarding the prevalence, biological characteristics and clinical significance of non-targeted dysplasia (NTD). We sought to determine the histological characteristics of NTD as a basis for future studies.

Design: Biopsies with NTD were identified on the basis of careful review of all available clinical data from IBD surveillance procedures performed with high-definition optics and/or chromoendoscopy between Jan. 2012 and Sept. 2016 including endoscopy reports and electronically recorded images. The slides were reviewed jointly by two GI pathologists. Biopsies classified by consensus as positive or indefinite but probably positive for dysplasia (IND-POS) were classified on the basis of configuration (Laine L, et al., Gastroenterology 2015;148:639) and cytoarchitectural features (Harpaz N, et al., submitted).

Results: Forty-six exams of 39 patients ( 28 UC, 10 Crohn's, 1 indeterminate) yielded 77 NTDs. Mean disease duration was $18.3 \mathrm{y}$. Thirteen $(29 \%)$ procedures were performed under chromoendoscopy. Twenty $(26.0 \%)$ NTDs were classified as IND-POS, 54 $(70.1 \%)$ as LGD and $3(3.9 \%)$ as HGD. Fifty-one (66.23\%) NTDs were flat, $10(13 \%)$ were superficially elevated and 16 were indeterminate. Morphologically, 56 NTDs $(73 \%)$ featured uncrowded crypts consistent with colonization of preexisting colorectal crypts by dysplastic epithelium. In the largest subgroup (41 NTDs), the cytoplasm simulated the repertoire of terminally differentiated colonic epithelial cells in normal colonic mucosa. Conventional adenomatous NTDs occurred in 18 cases, all in the immediate vicinity of inflammatory polyps. 
Conclusions: NTD is frequently flat and characterized by atypical epithelium that seems to colonize preexisting crypts, providing a rationale for their occult status under optimal endoscopic conditions. Conventional adenomatous NTD may escape detection because of adjacent mucosal irregularities. Future studies of NTD will aim to clarify its prevalence, biological characteristics and significance with respect to cancer risk.

\section{7}

Inflammatory Polyposis in Patients with Inflammatory Bowel

\section{Disease}

Yihong $R$ Ma, Huai-Bin Mabel Ko, Hongfa Zhu, Noam Harpaz, Alexandros D Polydorides. Icahn School of Medicine at Mount Sinai, New York, NY.

Background: Patients with inflammatory bowel disease (IBD), including ulcerative colitis (UC) and Crohn disease (CD), often develop inflammatory polyps (IPs) which can be large and/or multiple and may hinder successful endoscopic surveillance. The term inflammatory polyposis (IPS) has been used to indicate the presence of multiple or diffuse IPs, however exact criteria (number, size, or extent) have not been consistently employed. We sought to identify cases of IPS and determine their clinical outcomes, particularly in terms of the presence of incidental (previously unknown) neoplasia.

Design: Surgical resection specimens from patients with IBD were reviewed during a 10 year period (2006-2016) and cases of IPS were identified as those having diffuse, innumerable, carpeting, or multiple (more than 5) IPs described in the gross description or diagnostic comment, and/or seen in endoscopic images or gross specimen pictures. A control non-polyposis (NPS) group (with no, or at most 5, IPs) was identified from the same patient population. For patients with multiple surgeries, only the earlies identified resection was included. Clinicopathologic data (age, sex, IBD type, and highest grade of dysplasia) were recorded. Cases with dysplasia and/or carcinoma diagnosed on prior endoscopic biopsies were excluded.

Results: Fifty-three cases of UC patients with IPS were identified [35 (66\%) men, 18 (34\%) women, mean age 42 years (range: $19-73)]$ and $3(6 \%)$ had incidental colonic dysplasia (2 low-grade, 1 high-grade). No carcinomas were found. None of these variables were statistically significant when compared to a control group of 66 NPS UC cases, among which $2(3 \%)$ had low-grade dysplasia. Forty nine cases of CD patients with IPS were identified [30 (61\%) men, $19(39 \%)$ women, mean age 38 years (range: 19-69)] and 2 (4\%) had incidental low-grade dysplasia (one ileal, one colonic). These were similar to a control group of 154 NPS CD cases, where $5(3 \%)$ incidental neoplastic lesions were identified, including 2 colonic dysplasias (one low-grade, one high-grade) and 3 ileal adenocarcinomas.

Conclusions: IBD patients with resected IPS do not differ significantly in terms of age and sex compared to their NPS cohorts. Importantly, we found a low rate (5\%) of incidental dysplasia and no carcinomas in IPS cases, perhaps alleviating some of the anxiety due to incomplete surveillance in these patients.

738

Gene Expression Profiling of Appendiceal Goblet Cell Carcinoid

Tumors

Chelsea Maedler, Daniel Gaston, Nourah M Obaid, Thomas Arnason, Karen Bedard, Wei-Yuarn Huang. Dalhousie University, Halifax, NS, Canada.

Background: Appendiceal goblet cell carcinoid (GCC) tumors exhibit a wide spectrum of tumor differentiation ranging from well-differentiated to poorly differentiated adenocarcinoma ex-GCC. The gene expression profile of GCCs remains relatively unknown. Our aim is to identify novel biomarkers that can distinguish subgroups of GCCs.

Design: A total of 10 GCCs were selected, including 2 well-differentiated (group A), 4 signet-ring carcinoma ex-GCC (group B) and 4 poorly differentiated adenocarcinoma ex-GCC (group C). Total RNA, extracted from formalin-fixed paraffin embedded blocks, underwent RNA-sequencing analysis using Illumina HiSeq 2500. RNA-seq data was analyzed using a combination of HiSat2 for alignment, featureCounts for gene level count estimation, and DESeq2 for differential expression analysis and quality control. Differential expression was estimated for all pairwise comparisons. Genes were considered to be differentially expressed if they had a $>2$-fold change in either direction and a Benjami-Hochberg adjusted p-value $<0.05$. Lists of differentially expressed genes were then used for enrichment analysis using the DAVID platform (version 6.8). Results: Pairwise comparisons between tumor categories showed greater differences (by number of differentially expressed genes) between the A tumors and either the $\mathrm{B}$ or C tumors, which were more similar to each other (Table 1).

\begin{tabular}{|l|l|l|}
\hline Group Comparisons & \# of Differentially expressed genes & $\%$ of Genes \\
\hline A vs. B & 2757 & $5.1 \%$ \\
\hline A vs. C & 5870 & $10.8 \%$ \\
\hline B vs. C & 40 & $0.07 \%$ \\
\hline
\end{tabular}

Both the A vs B and A vs C comparisons showed enrichment in a number of functional categories including translational initiation, elongation, and termination; nonsensemediated decay; extracellular matrix organization and disassembly; focal adhesion; and basement membrane. Some of the most highly differentially expressed genes included those associated with ribosomes such as SNORD3A and RMRP (upregulated in C versus A tumors) and several long intergenic non-coding RNAs. Uncharacterized RNA genes (LINC00945, LOC100240734, LOC102724188, LOC105378656, LOC105378966) were downregulated in $\mathrm{C}$ versus A tumors. Intriguingly, several olfactory receptor genes were also among the most strongly upregulated genes in the $\mathrm{A}$ compared to the $\mathrm{C}$ group. Conclusions: This proof of concept study confirms differential gene expression between well-differentiated GCC and adenocarcinoma ex-GCC. This furthers our understanding of the GCC subgroups and their differing molecular characteristics.
$739 \quad$ Nuclear Exporter Protein CRM1/XPO1 a Novel Prognostic and Therapeutic Target in Gastric Cancer

Zaid Mahdi, Rahman Chaudhary, Irfana Muqbil, Amro Aboukameel, Ramzi Mohammad, Philip Philip, William Senapedis, Rafic Beydoun, Asfar Azmi. Wayne State University, School of Medicine, Detroit Medical Center, Detroit, MI; Wayne State University, School of Medicine, Detroit, MI; Karyopharm Therapeutics, Newton, MA.

Background: The high mortality rate associated with Gastric Cancer (GC) indicates the urgent need for identification of effective therapeutic markers. The nuclear exporter protein chromosome maintenance region 1 (CRM1) also known as exportin 1 (XPO1) is the exclusive exporter of nuclear proteins. CRM1/XPO1 is often over-expressed in different solid tumors and hematological malignancies. Excessive nuclear export through CRM1/XPO1 over-expression results in unusual shuttling of different critical tumor suppressor proteins causing their functional inactivation.

Design: A detailed analysis on the correlation of CRM1/XPO1 with inflammationmetaplasia-dysplasia-carcinoma sequence progression was performed using immunohistochemistry in 70 cases: (1) Ten cases for normal gastric mucosa, (2) Ten cases for stomach with intestinal metaplasia with and without inflammation, (3) Ten cases for mucosa with low-grade dysplasia (4) Ten cases for mucosa with highgrade dysplasia, (5) Ten cases for gastric adenocarcinoma and (6) Twenty cases for metastatic gastric carcinoma. Positive correlations between the CRM1 staining and the clinicopathologic features as well as survival were analyzed. Gastric cancer cell lines were exposed to our recently developed specific inhibitors of nuclear export (SINE) followed by cytotoxicity and molecular assays.

Results: CRM1/XPO1 served as prognostic marker for poor outcome as positive expression rates of CRM1 in GC with aggressive behavior were observed. Targeting CRM1/XPO1 using SINE (Selinexor, KPT-185, KPT-8602) or leptomycin B (+ve control) resulted in inhibition of $\mathrm{GC}$ cellular growth $\left(\mathrm{IC}_{50 \mathrm{~s}} \sim 300 \mathrm{nM}\right)$, induction of apoptosis $\left(>50 \%\right.$ apoptosis at $\mathrm{IC}_{50}$ doses $)$ and suppression of colony formation $(\mathrm{p}<0.01)$. Molecular analysis reveals nuclear retention of several important tumor suppressor proteins such HSP90 and FOXO3a, induction of pro-apoptotic proteins (Bax, PARP and caspase 3) cleavage and suppression of pro-survival factor Bcl-2. Lead SINE selinexor was well tolerated in mice upto a dose of $15 \mathrm{mg} / \mathrm{kg}$ p.o. Pre-clinical efficacy trial of selinexor in sub-cutaneous xenograft of $\mathrm{GC}$ is ongoing.

Conclusions: Our findings strongly demonstrate the potential of CRM1/XPO1 to serve not only as a prognostic marker but also a therapeutic marker in GC that warrants further clinical investigations.

740 Differential Expression and Subcellular Localization of Tumor Suppressor Maspin in Adenocarcinoma of Gastroesophageal Junction and Its Precursors

Zaid Mahdi, Sijana Dzinic, Semir Vranic, Faruk Skenderi, Margarita Bernardo, Shijie Sheng, Rafic Beydoun. Wayne State University, School of Medicine, Detroit, MI; University Clinical Center of Sarajevo and School of Medicine, University of Sarajevo, Sarajevo, Bosnia and Herzegowina; Barbara Ann Karmanos Cancer Institute, Detroit, MI.

Background: The most important etiologic factor in the development of adenocarcinoma of the gastroesophageal junction (AdGEJ) is Barrett's esophagus (BE). Difficulty regarding the diagnostic criteria of a number of cases of inflammation-metaplasia-low grade dysplasia (LGD)-high grade dysplasia (HGD)-AdGEJ sequence progression is mostly due to lack of reliable diagnostic markers. In contrast to tumor suppressor gene TP53 that is frequently mutated in AdGEJ hindering accurate diagnosis, an epithelial specific tumor suppressor maspin is 1) neither mutated nor deleted 2) is epigenetically and spatiotemporally regulated and 3) still remains the only endogenous inhibitor of histone deacetylase 1 (HDAC1). The purpose of this study is to investigate the role of maspin in the etiology of AdGEJ and its precursors.

Design: Maspin expression and subcellular localization were assessed in total of 50 cases of AdGEJ and its precursors using immunohistochemistry (clone G167-70; BD Biosciences). The normal GEJ mucosa was used as a control. Additionally, maspin was correlated with HDAC1 expression.

Results: Translocation of maspin from the nucleus to the cytosol was observed in all cases of intestinal metaplasia. Cytoplasmic localization was sustained in LGD and HGD. However, the expression and localization of maspin in AdGEJ was heterogeneous, ranging from high nuclear expression to complete maspin loss. There was no changes in HDAC1 expression or subcellular localization in AdGEJ precursors; however, HDAC1 expression was notably increased and inversely correlated with maspin expression in AdGEJ.

Conclusions: The early translocation of maspin from the nucleus to the cytoplasm in intestinal metaplasia indicates the possible role of maspin in etiology of AdGE and its potential role in aiding pathologists' to accurately diagnose BE due to sampling errors. Further studies are needed to correlate the observed heterogeneous maspin and HDAC1 expression in AdGEJ with patients' prognosis.

\section{The Role of Prolactin Receptor in Colonic Adenocarcinoma and Its Precursors}

Zaid Mahdi, Kerri Ann Latchminsing, Rafic Beydoun, Hayan Jaratli. Wayne State University, School of Medicine, Detroit, MI.

Background: Prolactin receptor (PR) is the cognate receptor of prolactin hormone that has the ability to bind and activates the intracellular Janus Kinase signalling pathway, leading to cell growth. Besides its known role in the tumorogenesis of breast, endometrial and cervical cancers, previous clinical data shows PR to be expressed in colorectal carcinoma (CRC). The aim of this study was to evaluate the level of expression of PR in preneoplastic conditions of (CRC) to better understand the main role of the hormone and its receptor in the initiation and/or the progression of (CRC). 
Design: We investigated a total of 50 cases of surgically resected polypectomies and colectomies for PR expression and compare them with normal colonic mucosa. Of 50 cases, 39 were polyps, ( 8 hyperplastic polyps (HP), 9 sessile serrated polyps (SSP), 8 tubular adenomas (TA), 5 tubulovillous adenoma (TVA), 5 tubular adenoma with high grade dysplasia and 4 pseudopolyps arising in ulcerative colitis mucosa) and 11 cases of CRC. Immunohistochemistry was performed using monoclonal PR antibody (Ab-1, Clone B6.2) and staining was scored for extent, intensity, and cellular location (membranous, cytoplasmic or both).

Results: Comparative analysis for PR expression were adjusted for baseline differences in which luminal membrane expression was considered as a baseline since it was shown to be positive by serendipity on all luminal crypts . On both polypectomy and colectomy specimens, cases with CRCs show intense (Score 4/5-5/5) membranous and cytoplasmic staining that ranges from focal to diffuse in $81 \%$ of cases. Conversely, tubular adenomas with high-grade dysplasia show a focal staining (score 2/4) in all cases. HPs, SSPs and TAs were all negative as well as cases of low grade dysplasia arising in the background of ulcerative colitis.

Conclusions: Our findings suggest that PR expression may be required during tumor progression and not during the early phase of tumor initiation. Besides, PR might be a helpful tool in microscopic differentiation between high-grade dysplasia and CRCs.

742 PD-L1 Expression in Post-Transplant Lymphoproliferative Disorder (PTLD) After Small Bowel Transplant

Abul Ala Syed Rifat Mannan, Hongfa Zhu, Alexandros D Polydorides, Noam Harpaz, Huai-Bin Mabel Ko. Mount Sinai Hospital, New York, NY.

Background: PTLDs are a heterogeneous group of lymphoid proliferations arising in the post-transplant setting. The majority are associated with EBV infection. Programmed cell death 1 (PD-1) and its ligand (PD-L1) are known to protect tissues from immunemediated damage, but can also allow tumors to evade immune destruction. Anti-PD-L1 immunotherapy has been shown to be effective in a variety of solid tumors. However, there is little data regarding PD-L1 expression in PTLDs, especially those occurring after intestinal transplantation (ITx). We sought to determine the incidence and clinicopathologic features of PTLD in ITx, including EBV status and PDL-1 expression. Design: ITx patients between 2005 and 2015 were identified from our pathology records. Clinical and pathologic details were reviewed. Slides were reviewed for confirmation of PTLD diagnosis and EBV status (EBER-ISH). IHC staining for PDL-1 (clone 22C3, Dako North America) was performed according to the manufacturer's recommendation. Staining was considered positive if $\geq 5 \%$ of tumor cells showed strong membranous staining, in accordance with the literature.

Results: A total of 69 patients (13 pediatric, 56 adults) received ITx. Nine developed PTLD (overall frequency 13\%; 5M, 4F; median age $26.5 \mathrm{y}, 5$ adult $/ 4$ pediatric). The median post-transplant interval was 8.5 months (range: $1-140 \mathrm{~m}$ ). Six of 9 patients (66.7\%) had experienced acute cellular rejection prior to the PTLD diagnosis. Initial diagnosis of PTLD was made in the GI tract ( $\mathrm{N}=7 ; 6$ in the small bowel graft), liver $(\mathrm{N}=1)$, and bone marrow $(\mathrm{N}=1)$. Histologically, $5(55.6 \%)$ cases were polymorphic and $4(44.4 \%)$ were monomorphic PTLD. EBER-ISH was positive in $8(88.9 \%)$ cases. Follow-up (mean 58.5m, range 1-109m) disclosed 4 deaths (44.4\%). PD-L1 IHC was positive in $6 / 9$ cases $(66.7 \%$, all showing at least $25 \%$ of cells positive). There was no significant correlation between PD-L1 expression and age, gender, time from transplant to PTLD diagnosis, monomorphic vs, polymorphic PTLD, EBV status and survival. Conclusions: PTLD is a common and serious complication of ITx in children and adults, mostly associated with EBV expression. The incidence in our series $(13.0 \%$ overall and $30.8 \%$ in children), is similar to that reported by others (Nalesnik et al.,Transplant Proc 2000;32:1213). PD-L1 was positive in the majority of our cases, but its expression did not correlate with any other clinical or histological features. Given the potential therapeutic benefit of anti-PD-L1 immunotherapy in PTLD, additional studies are warranted.

743 Unexpected Histopathological Findings in Appendectomy Specimens: An Institutional Experience

Abul Ala Syed Rifat Mannan, Malary Mani, Songyang Yuan, Huai-Bin Mabel Ko. Mount Sinai Hospital, New York, NY.

Background: Pathologic evaluation of the appendix after appendectomy is a routine practice. On occasion it can lead to unexpected but significant pathologic findings. The aim of this study was to determine the prevalence and types of incidental pathologic diagnoses found in routine appendectomy specimens at our institution.

Design: Appendectomy specimens submitted for pathologic evaluation (between January 2015 to December 2015) were retrospectively reviewed and pathologic diagnoses were recorded. All specimens were either open or laparoscopic appendectomies; appendices from colectomy specimens were excluded from the study.

Results: 562 appendectomy specimens were identified. Histological examination of the surgical specimen revealed acute suppurative appendicitis in $73.7 \%(414 / 562)$, and fibrous obliteration of the appendix in 7.7\% (43/562); in 5.5\% (31/562) the appendix was histologically normal. Incidental pathological diagnoses were noted in $13.2 \%(74 / 562)$ of appendectomy specimens. These included: neuroendocrine tumors (NET; $\mathrm{n}=13$; grade 1 NET: 11, and grade 2 NET: 2), mucinous cystadenomas ( $\mathrm{n}=13$ ), low grade appendiceal mucinous neoplasm $(n=8)$, hyperplastic polyp $(n=5)$, sessile serrated polyp/adenoma $(\mathrm{n}=9)$, tubulovillous adenoma $(\mathrm{n}=1)$, goblet cell carcinoid $(\mathrm{n}=2)$, mucinous adenocarcinoma $(n=3)$, adenocarcinoma $\operatorname{NOS}(n=1)$, carcinoma ex-goblet cell carcinoid $(\mathrm{n}=1)$, metastatic adenocarcinoma $(\mathrm{n}=1)$, granulomatous inflammation $(\mathrm{n}=3)$, diverticulitis $(\mathrm{n}=[$ thinsp $] 4)$, endometriosis $(\mathrm{n}=[$ thinsp $] 8)$, actinomycosis $(\mathrm{n}=[$ thinsp $] 1)$, and melanosis $(\mathrm{n}=1)$.

Conclusions: Our data revealed a large array of incidental findings in routine appendectomy specimens, which included inflammatory conditions, and various benign as well as malignant neoplasms. This highlights the importance of thorough microscopic examination of all appendectomy specimens to identify unsuspected conditions, which require further postoperative management. Gross intra-operative diagnosis alone is likely to miss a significant proportion of unexpected diseases.

\section{MSH3 and EMAST in Inflammatory Bowel Disease-Associated} Dysplasia and Carcinoma

Alexander Maris, Frank Revetta, Chanjuan Shi. Vanderbilt University Medical Center, Nashville, TN.

Background: Inflammatory bowel disease (IBD) increases risk of colorectal cancer (CRC) through an inflammation-dysplasia-carcinoma sequence. Elevated microsatellite alterations at selected tetranucleotide repeats (EMAST) in CRCs is thought to be a form of microsatellite instability that is initiated by inflammation and involved in CRC progression. Dysfunction in MSH3, a mismatch repair protein, may lead to EMAST in CRCs. Shifting of MSH3 from the nucleus to the cytoplasm may be one of the mechanisms underlying MSH3 deficiency. In this study, we explored the role of MSH3 and EMAST in inflammatory bowel disease-associated dysplasia and carcinoma. Design: 24 FFPE blocks from IBD colectomies had inflammation $(\mathrm{n}=20)$, low grade dysplasia (LGD; $n=20$ ), high grade dysplasia (HGD; $n=6)$, and CRC $(n=7)$. Seven additional IBD-associated CRCs were included from tissue microarrays. MSH3 expression was evaluated by immunohistochemistry (IHC). The location (nucleus versus cytoplasm) of MSH3 labeling was recorded. IHC scores of the nuclear labeling were obtained based on staining intensity and percent: negative $=0$, weak $=1$, moderate $=2$, and strong $=3 ; 0-4 \%=0,5-24 \%=1,25-49 \%=2,50-74 \%=3$, and $75-100 \%=4$. DNA was isolated from five IBD-associated CRC cases and analyzed by PCR sizing for EMAST Results: Weak $(\mathrm{n}=15,75 \%)$ to moderate $(\mathrm{n}=5,25 \%)$ nuclear MSH3 labeling was detected in inflamed colonic mucosa, mostly restricted to the deep crypts. Dysplasia was associated with an increase in staining intensity and/or percent. Fifteen of $20(75 \%)$ LGD and 5 of 6 (83\%) HGD lesions had moderate-to-strong nuclear labeling, which was more prominent in the deep crypts. Most CRCs had moderate-to-strong labeling, whereas two showed weak-to-moderate staining at the tumor surface while deeper, invasive glands clearly showed weaker nuclear staining and positive cytoplasmic staining. There was statistically significant increase in MSH3 expression in LGD (mean IHC score=7.3), HGD (mean=8.5), and CRC (mean=9.2) compared to inflammation without neoplasia (mean=3.7; $\mathrm{p}<.001$ ). PCR analysis of 5 CRCs showed no EMAST, including one with decreased nuclear MSH3 expression.

Conclusions: Altered MSH3 expression is present in IBD-associated dysplasia and invasive carcinoma, with most of the cases showing increased nuclear expression and a few cases with decreased nuclear and increased cytoplasmic expression in invasive carcinoma. Unlike sporadic CRCs, where about $60 \%$ of cancers have EMAST, EMAST may be rare in IBD-associated CRCs.

745 Aurora Kinase A Expression in Primary Resections of Gastrointestinal Stromal Tumors: Implications for Prognosis and Treatment Response

Daniel F Marker, Michael Landau. University of Pittsburgh Medical Center, Pittsburgh, PA.

Background: Aurora Kinase A (AURKA) is a serine/threonine kinase involved in mitotic regulation. Elevated expression of AURKA has been shown to be an independent predictor of recurrence or metastasis of gastrointestinal stromal tumors (GIST) and a poor prognostic marker in metastatic GIST. Clinical trials of inhibitors of AURKA have shown varying success for patients who have failed standard chemotherapy. The goals of our study were to correlate AURKA expression in primary GIST resections with clinical and pathologic features and to examine the effect of preoperative imatinib on AURKA expression.

Design: We identified 87 consecutive primary resections of GISTs $\geq 2 \mathrm{~cm}$ at our hospital from 2010 through 2015. We collected clinical and pathologic data and performed immunohistochemistry to assess the percentage of tumor cells expressing AURKA. Results: Eighteen (21\%) cases had metastasis at presentation or progressive disease after resection. The proportion of tumor cells expressing AURKA was higher in patients with metastatic or progressive disease compared to those without either $(10.7 \%$ vs. $4.5 \%$, $\mathrm{p}=0.0007)$. Of the patients who presented with metastatic disease, those who received preoperative imatinib (8 patients) had a lower proportion of tumor cells expressing AURKA than those who did not receive preoperative imatinib (7 patients) $(6.75 \%$ vs $16.14 \%$, $\mathrm{p}=0.015)$. Further characteristics stratified by percentage of tumor cells expressing AURKA using a cutoff of $8 \%$ are shown in Table 1.

\begin{tabular}{|l|l|l|l|}
\hline & $\begin{array}{l}\text { AURKA }< \\
8 \%(\mathrm{n}=60)\end{array}$ & $\begin{array}{l}\text { AURKA } \geq 8 \% \\
(\mathrm{n}=27)\end{array}$ & p-Value \\
\hline Mean Age, $\mathrm{y}$ & $63(\mathrm{SD}=12)$ & $61(\mathrm{SD}=13)$ & 0.76 \\
\hline Female Sex & $39(65 \%)$ & $13(48 \%)$ & 0.14 \\
\hline Mean Size, $\mathrm{cm}$ & $6.3(\mathrm{SD}=5.5)$ & $9.3(\mathrm{SD}=7.1)$ & 0.46 \\
\hline Mean Mitotic Count & $2.0(\mathrm{SD}=1.9)$ & $9.4(\mathrm{SD}=9.0)$ & $<0.0001$ \\
\hline Non-Gastric Location & $14(23 \%)$ & $12(44 \%)$ & 0.046 \\
\hline Risk of Malignant Potential (Miettinen) & & & 0.0007 \\
\hline High or Overtly Metastatic & $7(12 \%)$ & $14(52 \%)$ & \\
\hline Intermediate & $6(10 \%)$ & $4(15 \%)$ & \\
\hline Low & $25(42 \%)$ & $4(15 \%)$ & \\
\hline Very Low & $17(28 \%)$ & $3(11 \%)$ & \\
\hline $\begin{array}{l}\text { Could not be assessed (received neoadjuvant } \\
\text { imatinib and not overtly metastatic) }\end{array}$ & $5(8 \%)$ & $2(7 \%)$ & \\
\hline Metastatic at Presentation & $4(7 \%)$ & $11(41 \%)$ & $<0.0001$ \\
\hline
\end{tabular}


Conclusions: AURKA expression is often increased in tumors with high-risk features but seems to be decreased after treatment with imatinib. Therefore targeting AURKA with pharmacologic inhibitors may have limited utility in patients who have received imatinib.

746 Two-Stain Rather Than Four-Stain Immunohistochemica Screening in Colorectal Cancer May Fail to Detect Mismatch Repair Deficiency

Michael Markow, Rachel Pearlman, Christina A Arnold, Deborah Knight, Heather Hampel, Wendy L Frankel. The Ohio State University Wexner Medical Center and Comprehensive Cancer Center, Columbus, $\mathrm{OH}$.

Background: Universal tumor screening for Lynch syndrome (LS) in colorectal cancer (CRC) is recommended, and immunohistochemistry (IHC) for the mismatch repair (MMR) proteins (MLH1, MSH2, MSH6, PMS2) is a common method. The MMR proteins dimerize to form heterodimer complexes. If $\mathrm{MSH} 6$ is mutated, $\mathrm{MSH} 2$ binds with MSH3, resulting in sole absence of MSH6. If $\mathrm{MSH} 2$ is mutated, both MSH2 and MSH6 should be absent. MLH1 and PMS2 act in similar fashion. To reduce costs, some screen using only MSH6 and PMS2, with reflex to the partner stain if either are absent (two-stain method). We analyzed tumors with any MSH6 expression but absent MSH2 to determine if the two-stain method could miss $\mathrm{MSH} 2$ mutations.

Design: 1,300 CRC patients enrolled in the Ohio Colorectal Cancer Prevention Initiative underwent tumor screening with microsatellite instability testing and IHC. MSH2 and MSH6 stains were reviewed for proportion of positive cells and intensity of staining relative to internal control; $<5 \%$ staining was considered absent. Staining intensity was graded as: 1 , less than control with ambiguous nuclear staining; 2 , minimally less than control but with convincing nuclear staining; 3, equal to or greater than control. For patients with MMR deficiency, next-generation sequencing (NGS) of germline DNA for MMR genes was done (Coloseq, University of Washington). If germline was negative, NGS was done on tumor DNA to assess for double somatic mutations.

Results: Twelve patients $(0.9 \%, 12 / 1300)$ had absent MSH2 expression but weak MSH6 expression. Ten had LS due to pathogenic mutations in MSH2: five with grade 2 intensity and $15-30 \%$ of cells staining for MSH6, five with grade 1 intensity and $5-25 \%$ staining. Two had negative germline testing and double somatic mutations in MSH2: one with grade 1 intensity and 5\% staining, one with grade 2 intensity and $30 \%$ staining. A thirteenth case with absence of MSH2 was grade 3 with $30 \%$ of cells staining for MSH6; germline NGS is pending.

Conclusions: The two-stain method can miss patients with $M S H 2$ mutations when screening for LS. Convincing staining that is somewhat weaker than the control is difficult to evaluate and may be interpreted as present. Weak MSH6 expression can be retained in the absence of functional or antigenically intact MSH2, whether by forming an undiscovered heterodimer or through other means. If one uses the two-stain method, weak MSH6 staining should trigger reflex to MSH2 with consideration of MSI testing to identify a greater number of patients with LS.

747 Expression of Immuoregulators ID01, and PD-L1 in Gastrointestinal Stromal Tumors

Andres Matoso, Ayesha Siddique, Kara A Lombardo, Murray B Resnick, Ross Taliano, Li J Wang. Brown University, Providence, RI.

Background: The immune microenvironment is emerging as an important prognostic factor with potential therapeutic targets for various malignancies. Although the immunoregulators programmed death-ligand 1 (PD-L1) and indoleamine 2,3-dioxygenase (IDO1) have been studied in some tumor types, the significance of their expression in gastrointestinal stromal tumors (GISTs) is largely unknown.

Design: Tissue microarrays were constructed from 131 GISTs from our pathology files from 3/1996 to 3/2016. Immunohistochemistry for IDO1, and PD-L1 was performed. At least $10 \%$ of positive cells with moderate intensity staining was considered positive. Results were correlated with size, mitoses, and clinical followup. Tumor infiltrating lymphocytes (TIL) were counted using an image analysis software and reported in number of cells $/ \mathrm{mm}^{2}$.

Results: The mean age was 62 years (range 30-89) including 67 males and 64 females. The average size was $5.6 \pm 4.5 \mathrm{~cm}$ and the average mitotic count was $7.2 / 50 \mathrm{HPF}$. The tumor location included $89(68 \%)$ stomach (including 6 SDH deficient), $34(26 \%)$ small intestine, $3(2.2 \%)$ abdominal wall, $2(1.5 \%)$ rectum, $2(1.5 \%)$ colon and $1(0.8 \%)$ esophagus. The mean followup was 63 months. Nineteen $(14.5 \%)$ were malignant. Metastatic sites included mesentery $(n=8)$, liver $(n=6)$, lymph nodes $(n=3)$, falciform ligament $(n=1)$, and uterus $(n=1)$. Five patients died of disease with an average survival of 61 months (range $7-127)$. IDO1 was positive in $116(89 \%)$, including 14/19 (78\%) malignant and $102 / 112(91 \%)$ of benign tumors ( $\mathrm{p}=0.07)$. PD-L1 was positive in 89 $(68 \%)$, including $11 / 19(58 \%)$ malignant and $78 / 112(70 \%)$ benign tumors $(\mathrm{p}=0.4)$. PD-L1 positive tumors had a larger average size $(6.3 \pm 4.4$ vs. $4.4 \pm 3.4 ; \mathrm{p}=0.02)$ and higher number of mitoses/50HPF ( $8.9 \pm 5.4$ vs. $3.9 \pm 3.5 ; \mathrm{p}=0.006)$ than PD-L1 negative tumors. There was no significant difference in size or mitotic count between IDO1 positive and negative tumors. The average number of CD8-positive TILs was $168 \pm 35$ / $\mathrm{mm} 2$ in PD-L1 positive tumors with staining of tumor cells. PD-L1 positive tumors (staining of tumor cells) had significantly higher numbers of PD-L1 positive TILs than PD-L1 negative tumors ( $113 \pm 21$ vs. $104 \pm 18 ; \mathrm{p}<0.001)$.

Conclusions: The majority of GISTs express PD-L1 and IDO1. Expression of PD-L1 was associated with increased tumor size and higher mitotic activity. PD-L1 and IDO1 could play a significant role in the tumor biology of GISTs, and immunotherapy targeting one or both may provide novel treatment options.
748 Deep Sequencing of Intrahepatic Cholangiocarcinoma, Extrahepatic Cholangiocarcinoma and Klatskin Tumors

Daiva Mattis, Nancy M Joseph, Iwei Yeh, Eric Talevich, Courtney Onodera, Aras N Mattis. University of California San Francisco, San Francisco, CA.

Background: Cholangiocarcinoma represents $\sim 3 \%$ of all gastrointestinal neoplasia and has poor prognosis with no functional therapy beyond surgery. Recent molecular profiling by next generation sequencing (NGS) of a limited number of samples has identified specific alterations associated with intrahepatic cholangiocarcinoma (ICC) and extrahepatic cholangiocarcinoma (ECC). To expand our understanding of the pathogenesis of ICC, ECC, and to characterize Klatskin tumors, we performed capturebased NGS on archival clinical cases.

Design: 6 Klatskin, 6 ECC, and 9 ICC cases were identified in the UCSF archives. All slides and pathology reports were reviewed for consistency. DNA was extracted from both normal and tumor tissue for each case. NGS was performed, targeting exons of over 500 cancer genes and select introns of 40 genes. Duplicate reads were removed computationally for accurate allele frequency determination and copy number calling. Single nucleotide variants (SNVs), insertions/deletions, structural rearrangements, and copy number alterations were evaluated.

Results: IDH1 mutations and focal amplifications were only present in ICC samples $(55 \%)$. ECC samples generally harbored mutations in multiple tumor suppressor genes including CDKN2A, TP53, ARID1A, TGFBR2, and SMAD4, similar to prior studies. Klatskin tumors, like ECC showed activating mutations in oncogenes with $50 \%$ of cases having KRAS mutations, and one-third of cases having rearrangements in either FGFR2 or MET.

Conclusions: This is the first study to deep sequence and characterize Klatskin tumors in comparison to ICC and ECC. In this study we confirmed that ICCs often contain oncogenic mutations such as IDH1 and KRAS. In contrast ECCs demonstrated multiple mutations in tumor suppressor genes. Klatskin tumors, like ICC, contained activating alterations of oncogenes including KRAS, FGFR2, and MET, but unlike ICC, did not harbor any mutations in IDH1. A small percent of both Klatskin and intrahepatic cholangiocarcinomas had rearrangement mutations in FGFR2 or MET, but none in ECCs. Our findings suggest that Klatskin and intrahepatic tumors are largely oncogene driven, whereas distal tumors are tumor suppressor driven. To our knowledge this is the first study to perform deep sequencing on Klatskin tumors with important comparisons to ECCs and ICCs.

\section{Detection of Clonal Evolution in Solid Tumors by Ultra-Deep} Sequencing of Plasma Cell-Free Nucleic Acid Using Miseq

Meenakshi Mehrotra, Rajesh R Singh, Wei Chen, Bedia A Barkoh, Carmen Behrens, Keyur P Patel, Mark J Routbort, Russell R Broaddus, L J Medeiros, Scott Kopetz, Ignacio I Wistuba, Rajyalakshmi Luthra. The University of Texas MD Anderson Cancer Center, Houston, TX.

Background: Genetic heterogeneity in cancers is a major challenge in delivering precision therapy based on mutational profiling of a tumor biopsy specimen. Detection of mutations in circulating cell-free DNA (cfDNA) using next generation sequencing (NGS) allows monitoring of clonal heterogeneity in a non-invasive manner. In this study, our aim is to show the utility of cfDNA ultra-deep sequencing combined with a custom bioinformatics pipeline for monitoring clonal heterogeneity in patients with advanced solid tumors.

Design: Plasma samples from 23 patients with 7 primary and 16 metastatic advanced carcinomas including colon $(n=9)$, endometrium $(n=6)$, ovary $(n=4)$, prostate $(n=2)$, thyroid $(n=1)$, and breast $(n=1)$ with known mutation status in tissue determined using the NGS-based Ampliseq cancer Hotspot panel v2 were selected. The median time from tumor biopsy to plasma collection was 371 days (range, 4-3,373 days). Plasma cfDNA was extracted using QIAamp circulating nucleic acid kit. 20ng of cfDNA was used for targeted amplification and library preparation using Accel amplicon 56G oncology panel (Swift Biosciences) and sequenced using Miseq sequencer (Illumina). Sequencing data generated were analyzed by a custom-built bioinformatics pipeline. Mutations and new clones detected in plasma cfDNA were confirmed by droplet digital PCR based on assay availability (KRAS: G12V, G12C, G12D, G12S; PIK3CA p.E545K).

Results: Ultra-deep sequencing of cfDNA detected 31/34 (91\%) expected mutations at $6,000 \mathrm{X}$ average sequencing depth. The mean variant allelic fraction for mutations detected in tissue specimens was $33.4 \%$ (range, 1- 86\%) and in plasma 19.2\% (range, $0.2-61 \%)$. Droplet digital PCR confirmed 8 expected and 1 new clone in plasma (PIK3CA p.E545K). Eleven additional mutations were detected in plasma: 3 KRAS p.Q61H (range, $2 \%-14 \%$ ), TP53 p.C275Y (10\%), TP53 p.R273H (6.1\%), TP53 p.A276G (1.2\%), APC dupApT1556fs*3 (32.9\%), PIK3CA p.E545K (39.1\%), EZH2 p.Y646F $(5.7 \%)$ and $E G F R$ p.F712S (1\%).

Conclusions: We were able to detect $91 \%(31 / 34)$ of expected mutations and additional new clones by ultra-deep sequencing of cfDNA. Detection of additional mutations in cfDNA not observed in the single site tumor biopsy specimens allows early detection of emerging clones and may facilitate adoption of alternative therapeutic strategies for disease management and treatment of patients with solid tumors.

750 EPB41L5 and PDK2 Are Biomarkers of Metastasis in Pancreatic Neuroendocrine Tumors

Tania Mendoza, Steven A Eschrich, Kevin Neil, Jonathan R Strosberg, Nasir Aejaz, Domenico Coppola. H. Lee Moffitt Cancer Center and Research Institute, Tampa, FL; Eli Lilly and Company, Indianapolis, IN.

Background: The malignant behavior of pancreatic neuroendocrine tumors (PNETs) is currently unpredictable. PNETs are indolent but have malignant potential if left untreated. Unfavorable prognostic factors include high grade, large size and metastasis. PNETs often metastasize to the liver. Increased tumor burden in the liver is common cause of death in PNET patients. Molecular biomarkers predicting metastasis in PNETs are needed to improve treatment selection 
Design: Five primary PNET from 5 patients (mean age $66 ; 3 \mathrm{M} / 2 \mathrm{~F}$ ), 6 primary PNET with synchronous or metachronous metastasis (M-PNET) (mean age 59,3M/3F) and 6 PNET metastatic to liver (ML-PNET) from the same six patients were selected. RNA extracted from these frozen samples was analyzed on Affymetrix U133 2.0 gene chip. Data were normalized using IRON. A Student's t test was used to identify differentially expressed genes in PNETs vs. M-PNETs, along with a 2 -fold difference in mean. Based on statistical analysis and extensive literature review two genes were selected as potential predictor markers for metastasis

Results: 1,483 probe sets, representing 1,036 genes, were found to be differentially expressed $(\mathrm{p}<0.05)$ between PNETs vs. M-PNETs. One hundred and ninety-five probe sets had $\geq 2$ fold change; 75 of these probe sets were overexpressed in M-PNET suggesting possible biomarkers of metastasis. Among the significant genes, EPB41L5 $(\mathrm{p}=0.005)$ and PDK2 $(\mathrm{p}=0.004)$ had the smallest $\mathrm{p}$ value and largest fold-change between PNET and M-PNET. Expression of EPB41L5 $(\mathrm{p}=0.33)$ and PDK2 $(\mathrm{p}=0.22$ were not statistically different between M-PNET and ML-PNET paired samples. PDK2 or pyruvate dehydrogenase kinase isoenzyme 2 inactivates the mitochondrial pyruvate dehydrogenase complex through serine phosphorylation. EPB41L5 an erythrocyte membrane protein is involved in cell polarity processes. Validation of these gene products is under investigation on tissue microarray containing an independent set of PNET with and without metastases

Conclusions: We report for the first time that EPB41L5 and PDK2 are potential biomarkers of PNET metastasis. Our result warrant further investigation. If confirmed, EPB41L5 and PDK2 be used to improve clinical decision making in patient with PNETs

751 Loss of YAP-1 Expression in Gastrointestinal Neuroendocrine Carcinomas

James A Miller, Tamara L Lotan. Johns Hopkins, Baltimore, MD.

Background: The Hippo pathway is involved in regulation of organ size and includes the Hippo and Warts phosphorylases, and the transcriptional co-activator, Yorkie (YAP1). Hippo signaling is upregulated in many cancers and correlated with poor outcomes. We developed a gene expression signature to distinguish neuroendocrine carcinoma and adenocarcinoma and noted that loss of YAP1 gene expression was common in lung and prostatic neuroendocrine carcinoma. We tested whether immunohistochemistry for this marker might be useful in gastrointestinal neuroendocrine carcinomas.

Design: Ten tissue microarrays of gastrointestinal neuroendocrine carcinomas, including 111 unique cases, were stained for YAP1, synaptophysin, and chromogranin. YAP1 staining was considered positive if cytoplasmic or nuclear staining was observed in the tumor cells on any tumor core, and YAP1 was interpreted as negative if no cytoplasmic or nuclear staining was seen in tumor cells on any tumor core in the presence of intact stromal/endothelial staining. Synaptophysin and chromogranin were interpreted as positive if any cytoplasmic staining was seen on any tissue core, while they were interpreted as negative if no cytoplasmic staining was seen in tumor cells on any tumor core.

Results: One hundred seven (96.4\%) of the cases had documented metastasis, $2(1.8 \%)$ were solitary tumors, and the presence of metastises could not be determined in $2(1.8 \%)$ cases. Fifty two $(46.8 \%)$ primary tumors, and $100(90.0 \%)$ metasteses were examined (with both sites examined in 41 cases). Primary sites included pancreas (46, 47\%), liver $(4,4 \%)$, small bowel $(37,38 \%)$, colon $(8,8 \%)$, and stomach $(2,2 \%)$. Metastatases included liver $(105,98.1 \%)$, soft tissue $(2,2 \%)$, lymph nodes $(1,0.9 \%)$, ovary $(1,0.9 \%)$, and not-specified $(2,2 \%)$. YAP1 was lost in $47(42 \%)$ of cases and was retained in 64 $(58 \%)$ of cases. YAP1 was lost in $21(40.4 \%)$ of the 52 primaries, and retained in 31 $(59.6 \%)$ primaries. YAP1 was also lost in $47(47 \%)$ of the 100 metastases and retained in $53(53 \%)$ metastases. Synaptophysin was positive in $109(95.5 \%)$ of cases, and negative in $1(0.9 \%)$ case. YAP1 staining was available in both the primary and metastasis in 41 cases. Chromogranin was positive in $106(96 \%)$ cases, and negative in $4(4 \%)$ cases. Conclusions: CONCLUSIONS: The Hippo pathway is frequently downregulated in gastrointestinal neuroendocrine carcinomas. Future studies to determine if there is a relationship between YAP-1 loss and neuroendocrine differentiation, as well as the association with prognosis may be of interest.

752

Tubulovillous Adenomas with Serrated Features Are Associated with a Higher Risk of Advanced Neoplasia on Follow-Up

Mohamed Mostafa, Christopher Hartley, Catherine Hagen. Medical College of Wisconsin, Milwaukee, WI; University of Wisconsin Hospital and Clinics, Madison, WI. Background: Tubulovillous adenoma (TVA) and traditional serrated adenoma (TSA) represent precursor lesions for two discrete pathways of colorectal carcinogenesis. Occasionally, TVA show focal features resembling TSA. Currently, the biological significance of this histologic variant of TVA is unknown. The goal of this study was to assess patient outcome in a group of patients with tubulovillous adenomas with serrated features (TVASF) as compared to conventional TVA.

Design: A set of 225 consecutive TVA from 207 patients which had previously been reviewed for histologic features of TVASF was used for this study. Data from all previous and subsequent colonoscopy procedures including polyp number, location, phenotype, and degree of neoplasia was recorded via chart review. Advanced neoplasia on prior or follow-up endoscopy was considered as TVA or at least high grade dysplasia (HGD). Results: 37 TVA (16.4\%) from 36 patients showed serrated features. TVASF were significantly more-likely to have HGD or greater arising in the polyp $(59.5 \% \mathrm{vs}$. $16.5 \%, \mathrm{p}<0.01)$. Patients with TVA showed a trend of having more synchronous tubular adenomas $(43.3 \%$ vs. $25 \%$, p $=0.06) .70$ patients had at least one prior colonoscopy and 94 patients had at least one follow-up colonoscopy. Patients with TVASF were significantly older $(70.0$ vs. $64.4, \mathrm{p}=0.007)$ and were more likely to have had a prior endoscopy with advanced neoplasia ( $33.3 \%$ vs. $14.0 \%, \mathrm{p}=0.013$ ). Patients with TVASF were significantly more likely to have advanced neoplasia on follow-up colonoscopy $(\mathrm{p}=0.04, \mathrm{HR}=2.3,95 \% \mathrm{CI} 1.03-8.31)$. When patients with previous advanced neoplasia were excluded from analysis, patients with TVASF were still significantly more likely to have advanced neoplasia on follow-up ( $\mathrm{p}=0.02, \mathrm{HR}=2.7,95 \% \mathrm{CI} 1.29-11.70$ ).
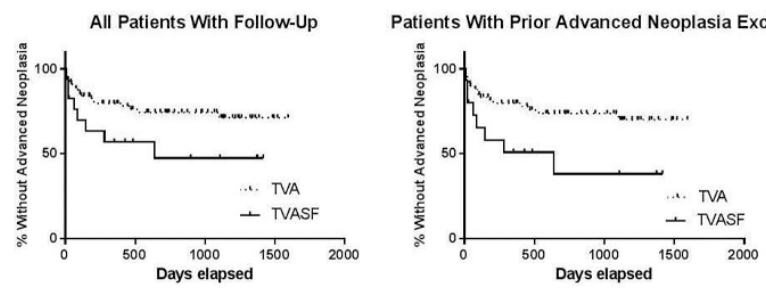

Conclusions: TVASF are frequently associated with prior and subsequent advanced neoplasia. Patients with TVASF may require more aggressive colonoscopic follow-up, but further longterm studies are necessary.

\section{Evaluation of the Lower Histologic Threshold for Gastric Graft versus Host Disease}

Mohamed Mostafa, Christopher Hartley, Catherine Hagen. MCW, Milwaukee, WI; University of Wisconsin, Madison, WI.

Background: The minimum histologic criteria for diagnosis of gastrointestinal (GI) graft-versus-host disease (GVHD) remains debated. Recent studies have re-examined histologic criteria for diagnosis of GVHD in colon biopsies but data for gastric biopsies is largely lacking. The aim of this study was to retrospectively review a cohort of gastric biopsies taken to evaluate for GVHD and correlate histologic findings with clinical data in order to define a lower diagnostic threshold for gastric biopsies.

Design: Our pathology database was retrospectively searched for gastric biopsies taken to evaluate for GVHD from 2008-2013. Biopsies from solid organ transplant patients and asymptomatic patients were excluded. Gastric biopsies were blindly reviewed for the maximum number of apoptotic bodies per 10 contiguous gastric pits (Ap./10), presence of gastric pit dropout, and presence of $>1$ apoptotic body per biopsy fragment (NIH guidelines). Clinical information was collected through chart review.

Results: The study group consisted of 65 gastric biopsies from 52 stem cell transplant patients (M:F 1.5:1, mean age 51.7). The mean number of biopsy fragments per specimen was 4.1 and the mean number of levels examined was 9.0. The mean Ap./10 for all biopsies was 1.8 (range $0-8$ ). 19 biopsies $(29 \%)$ had $>1$ apoptotic body per biopsy fragment. Only 5 cases $(7.7 \%)$ had evidence of gastric pit dropout. Immunosuppression was initiated after 45 of the biopsies $(69.2 \%)$. Treated patients had significantly higher Ap. $/ 10$ compared to untreated patients $(2.1$ vs. $0.9, \mathrm{p}=0.01)$ as did patients with evidence of GVHD in other GI biopsies (2.2 vs. 1.3, $\mathrm{p}=0.04)$. Presence of $>1$ Ap. per biopsy fragment was not significantly associated with treatment $(\mathrm{p}=0.06)$ or evidence of GVHD at other sites (GI, $p=0.15$; extraintestinal, $p=0.08$ ). However, when the $\mathrm{NIH}$ guidelines were combined with presence of at least $2 \mathrm{Ap}$./10 this was significantly associated with treatment $(\mathrm{p}=0.047, \mathrm{OR}=8.5)$ and evidence of $\mathrm{GVHD}$ at other $\mathrm{GI}$ sites $(\mathrm{p}=0.04, \mathrm{OR}=5.3)$. Conclusions: Gastric biopsies rarely show pit dropout and tend to show only subtle features of GVHD consisting of rare apoptosis. Combining the NIH guidelines with presence of at least $2 \mathrm{Ap}$./10 is significantly correlated with treatment and presence of GVHD at other GI sites. Larger studies could help confirm the findings reported here.

\section{$754 \quad$ EBV and Microsatellite Instability Can Occur in a Subset of Lymphocyte-Rich Intrahepatic Cholangiocarcinomas}

Taofic Mounajjed, Hee Eun Lee, Tsung-Teh Wu. Mayo Clinic, Rochester, MN.

Background: Clinicopathologic characteristics of lymphocyte-rich intrahepatic cholangiocarcinoma (LRICC) have not been well described. Lymphocyte-rich carcinoma in gastrointestinal tract has been associated with EBV and/or microsatellite instability (MSI). However, such association in cholangiocarcinoma (ICC) has not been studied in detail. We aimed to evaluate clinicopathologic characteristics of LRICC and its EBV and MSI status.

Design: The density of tumor infiltrating lymphocytes (TIL) was graded in 154 ICCs $[0=\leq 5 \mathrm{TIL} / \mathrm{HPF}(\mathrm{n}=114), 1+=6-10 \mathrm{TIL} / \mathrm{HPF}(\mathrm{n}=6), 2+=11-15 \mathrm{TIL} / \mathrm{HPF}(\mathrm{n}=18), 3+$ $=\geq 16 \mathrm{TIL} / \mathrm{HPF}(\mathrm{n}=16)]$; cases with $3+$ and 0 were regarded LRICC and lymphocytepoor ICC (LPICC), respectively. Fifteen LRICCs were compared to the same number of randomly selected LPICCs. EBV in situ hybridization and immunostains for MLH1, PMS2, and MSH2 were performed. Cases were classified into proficient mismatch repair (pMMR: MLH1, PMS2 and MSH2 all intact) and deficient MMR (dMMR: if any loss). Clinical data were correlated with EBV and MMR status, and with pathologic features. Results: Median age of the LRICC and LPICC group was 59 years (range 31-83 years) and 67 years (range, 55-74 years), respectively. Histologic type was adenocarcinoma (ADCA) in all cases except one carcinosarcoma (LRICC group) and one combined hepatocellular carcinoma and ICC (LPICC group). Two LRICCs and 3 LPICCs showed low histologic grade (LG) ADCA and remaining cases high grade (HG) ADCA. Tumor AJCC stage of the LRICC group was T1 $(n=9)$, T2 $(n=4)$, T3 $(n=2)$, N0 $(n=7)$, N1 $(\mathrm{n}=3)$, and $\mathrm{Nx}(\mathrm{n}=5)$, compared to T1 $(\mathrm{n}=9), \mathrm{T} 2(\mathrm{n}=6), \mathrm{T} 4(\mathrm{n}=1), \mathrm{N} 0(\mathrm{n}=11)$ and $\mathrm{Nx}$ $(n=4)$ in LPICC group. Two LRICCs showed distant metastasis compared to none in the LPICC group. The LRICC group tended to have a longer disease-free survival than the LPICC (mean \pm SD, $76.7 \pm 16.9$ vs $61.0 \pm 20.7$ months; $\mathrm{p}=0.276$, log-rank test). One (6.7\%) LRICC (HG ADCA with pMMR and T1N1M1) showed diffuse EBV positivity as compared to none in LPICC. The patient died of disease 32 months after diagnosis. Two (13.3\%) LRICCs (HG ADCA with T1N1M0 and LG ADCA with T1NxM0) showed loss of MLH1 and PMS2 representing dMMR, compared to none in LPICC. Both patients were alive without disease for 69 and 128 months, respectively. 
Conclusions: Our findings indicate that EBV infection and MSI may play a role in carcinogenesis of intrahepatic cholangiocarcinoma, in particular in a subset of lymphocyte-rich tumors.

755 Verrucous Carcinoma of the Esophagus Shares a Methylation Profile with Usual Esophageal Squamous Carcinoma

Prasuna Muppa, William R Taylor, Tracy C Yab, Douglas W Mahoney, John B Kisiel, David A Ahlquist, Thomas Smyrk. Mayo Clinic, Rochester, MN.

Background: Verrucous carcinoma of the esophagus (VCE) is a rare entity characterized by a bulky intraluminal mass with bland cytology and a pushing border. Endoscopic biopsies tend to be superficial and non-diagnostic, and even deep biopsies often show only broad pegs of squamous epithelium pushing into the esophageal wall, without architectural and cytologic features of usual squamous cell carcinoma (SCC). Molecular markers may aid in detection of VCE, but have not been reported. We have previously identified novel methylated DNA markers for both esophageal adenocarcinoma (EAC) and squamous cell carcinoma (ESCC) on whole methylome sequencing.

Design: In this exploratory study, we assessed the informativeness of selected novel methylated DNA markers for EAC and ESCC for application in the detection of VCE. We identified and histologically confirmed 10 patients with VCE. Demographic and clinical data were obtained from the medical record. On DNA extracted from macrodissected paraffin tissue, 16 selected methylated DNA markers were assayed using real time quantitative methylation specific PCR (qMSP). We also analyzed 22 normal esophageal samples by qMSP as negative controls. A multivariate regression model was used to analyze methylation data significantly associated with VCE. Results:
Normal

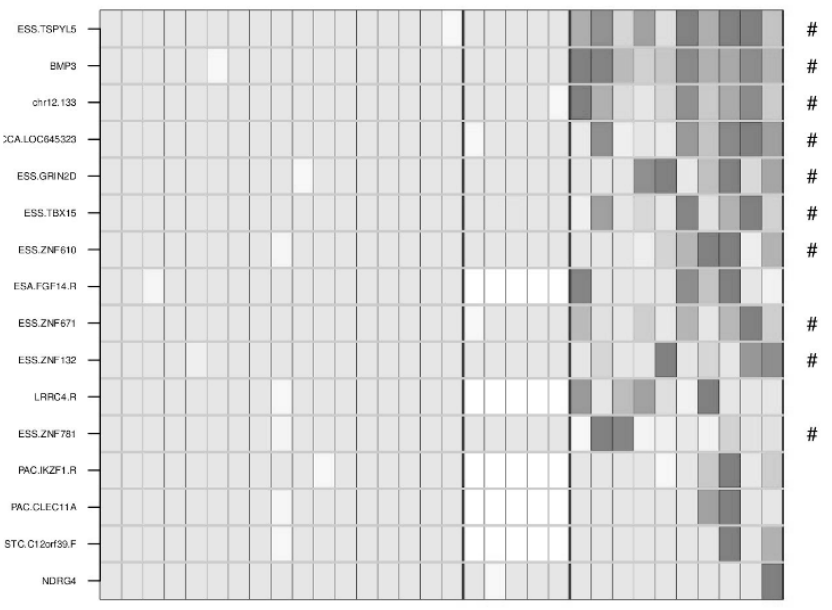

Subject
The above figure summarizes the percent methylation of selected genes assayed in VCE and normal samples. Significant hypermethylation was noted in 10 genes reflecting a pattern that we have seen with ESCC (as highlighted by \# symbol). These genes were not methylated in healthy esophageal tissue. Also, genes that we have found to be exclusively hypermethylated in EAC were not methylated in VCE. Methylated BMP3 and TSPYL5 perfectly discriminated $\mathrm{VCE}(\mathrm{AUC}=1.0 ; \mathrm{p}$-value $=0.0$ ), and methylated $Z N F 781, T B X 15$, and $c h r .133$ displayed very high discrimination as well (AUCs $=0.991,0.986,0.936$; $\mathrm{p}$-values $=0,0,0$, respectively). Other discriminant methylated gene markers included LRRC4, LOC645323, and ZNF610 in VCE tissue (p-values $=0,0,0$, respectively). Conclusions: We have identified highly discriminant methylated DNA markers for $\mathrm{VCE}$, a rare entity with no known molecular or immunohistochemical markers. Limited methylation profiling suggests that VCE is more similar to ESCC than to EAC.

$756 \quad$ Clinical Manifestations of Pathologic Site-Based Classification of Ampullary Carcinomas (ACs): An Analysis of 107 Resected ACs Demonstrates Distinct Clinicopathologic Associations

Takashi Muraki, Michelle D Reid, Steven Keilin, Serdar Balci, Nobuyuki Ohike, Takuma Tajiri, Yue Xue, Bahar Memis, Aarti Sekhar, Pardeep Mittal, Alyssa Krasinskas, David Kooby, Shishir Maithel, Juan M Sarmiento, Volkan Adsay. Emory Uni., Atlanta, GA. Background: Ampullary cancers (ACs) are now classified under 4 categories based on their origin from the anatomic compartments of ampulla and corresponding growth pattern (CAP synoptic protocol, Jan, 2016). The pre-operative clinical/imaging correlates of these categories are not known.

Design: 107 resected ACs with preoperative imaging available (24 also with endoscopic data) were analyzed and contrasted with pancreatic adenocarcinomas (PC; 61 radiologically and 500 prognostically).

Results: ACs had a significantly better prognosis than PC. Clinicopathologic correlates of the AC subtypes are documented in the tables.

\begin{tabular}{|c|c|c|c|c|c|}
\hline & $\begin{array}{l}\text { Age, } \\
\text { F/M }\end{array}$ & $\begin{array}{l}\text { Distinguishing } \\
\text { clinical features }\end{array}$ & $\begin{array}{l}\text { More specific } \\
\text { imaging findings }\end{array}$ & $\begin{array}{l}\text { Endoscopic } \\
\text { findings }\end{array}$ & $\begin{array}{c}5 \mathrm{yr} . \\
\text { surval } \\
(\%)\end{array}$ \\
\hline $\begin{array}{c}\text { Ampullary- } \\
\text { ductal }(\mathrm{n}=33, \\
31 \%)\end{array}$ & $\begin{array}{l}66 \\
0.7\end{array}$ & $\begin{array}{l}{ }^{*} \mathrm{H} / \mathrm{O} \text { of alcohol } \\
\text { abuse was common } \\
\text { ( } 21 \% \text {, vs } 5 \% \text { in } \\
\text { other types). }\end{array}$ & $\begin{array}{l}\text { *Similar to } \\
\text { PC with duct } \\
\text { dilatation (mean, } \\
5.3 \mathrm{~mm} \text { ) and } \\
\text { parenchymal } \\
\text { atrophy (mean, } \\
12.2 \mathrm{~mm} \text { of the } \\
\text { body). }\end{array}$ & $\begin{array}{l}\text { *Non-exposed } \\
\text { protrusion } \\
\text { (with/without a } \\
\text { central dimple, } \\
\text { or unlceration). } \\
(\mathrm{n}=7)\end{array}$ & 45 \\
\hline $\begin{array}{c}\text { (Peri) ampullary- } \\
\text { duodenal(n=19, } \\
18 \%)\end{array}$ & $\begin{array}{l}59, \\
1.1\end{array}$ & $\begin{array}{l}\text { *Younger patients. } \\
{ }^{*} \text { Common pain } \\
(53 \% \text {, vs } 29 \%) . \\
\text { *Less jaundice } \\
(53 \% \text {, vs } 85 \%) . \\
\text { *Lowest pre-ope } \\
\text { hemoglobin (mean, } \\
9.6 \mathrm{mg} / \mathrm{dl} \text {, vs } 11.6) .\end{array}$ & $\begin{array}{l}\text { *Variable } \\
\text { pancreatic duct } \\
\text { diameter (range; } \\
0-11 \mathrm{~mm}) \text {. }\end{array}$ & $\begin{array}{l}* \text { Overt white } \\
\text { granular tumors } \\
\text { on the duodenal } \\
\text { mucosa } \\
\text { surrounding the } \\
\text { papilla. }(\mathrm{n}=7)\end{array}$ & 41 \\
\hline $\begin{array}{c}\text { Intra-ampullary } \\
\text { tubular } \\
\text { neoplasm- } \\
\text { associated(n=14, } \\
13 \%)\end{array}$ & $\begin{array}{l}63, \\
0.6\end{array}$ & $\begin{array}{l}\text { *Commonly } \\
\text { associated with } \\
\text { cholangitis }(35 \% \text {, } \\
\text { vs } 9 \%) \text { and } \\
\text { pancreatitis }(21 \% \text {, } \\
\text { vs } 3 \%) \text {. } \\
\text { *Minimal weight } \\
\text { loss (BMI }>25 \% \text {; } \\
92 \% \text {, vs } 55 \%) \text {. }\end{array}$ & $\begin{array}{l}\text { * Most lacked } \\
\text { pancreatic duct } \\
\text { dilatation (mean, } \\
2.8 \mathrm{~mm} \text { ). }\end{array}$ & $\begin{array}{l}\text { *Swollen intact } \\
\text { mucosa, with } \\
\text { sphincterotomy } \\
\text { exposing white } \\
\text { small nodular } \\
\text { granular lesion. } \\
(\mathrm{n}=4)\end{array}$ & 64 \\
\hline $\begin{array}{l}\text { Mixed/NOS } \\
(\mathrm{n}=41,38 \%)\end{array}$ & \multicolumn{5}{|c|}{$\begin{array}{l}\text { Non-specific other than pancreatic cysts }>1 \mathrm{~cm} \text { only in this subtype }(\mathrm{n}=6 \text {, } \\
\qquad 15 \%) .\end{array}$} \\
\hline
\end{tabular}

\begin{tabular}{|c|c|c|c|c|c|c|c|}
\hline & \multicolumn{3}{|c|}{ Pre-operative diagnosis } & \multicolumn{3}{c|}{ Pathological findings } \\
\hline & $\begin{array}{c}\text { AC } \\
(\%)\end{array}$ & $\begin{array}{c}\text { PAC } \\
(\%)\end{array}$ & $\begin{array}{c}\text { PC/BC } \\
(\%)\end{array}$ & $\begin{array}{c}\text { Duodenal } \\
(\%)\end{array}$ & $\begin{array}{c}\text { Size of } \\
\text { overall } \\
\text { tumor/ } \\
\text { inv. (cm) }\end{array}$ & $\begin{array}{c}\text { LVI/PNI } \\
(\%)\end{array}$ & $\begin{array}{c}\text { LN } \\
\text { mets } \\
(\%)\end{array}$ \\
\hline Ampullary-ductal & 39 & 27 & 24 & 3 & $1.8 / 1.8$ & $73 / 63$ & 61 \\
\hline $\begin{array}{c}\text { (Peri) ampullary- } \\
\text { duodenal }\end{array}$ & 26 & 16 & 21 & 37 & $4.8 / 2.8$ & $47 / 42$ & 53 \\
\hline $\begin{array}{c}\text { Intra-ampullary tubular } \\
\text { neoplasm-associated }\end{array}$ & 71 & 7 & 22 & 0 & $2.3 / 0.9$ & $36 / 31$ & 36 \\
\hline PAC; Peri-ampullary ca, PC; Pancreatic head ca, BC; Bile duct ca \\
\hline
\end{tabular}

Conclusions: The 4 site-based categories of ampullary carcinomas recognized in the CAP protocol have distinctive imaging and clinical characteristics in addition to specific pathologic features and biologic behavior.

757

Loss of Expression of CDX2 Is Not a Useful Method for Detecting Epigenetic Gene Alterations

Eva Musulen, Anna Martinez-Cardus, Sebastian Moran, Aurelio Ariza, Miquel Angel Carrasco, Manel Esteller. Hospital Universitari Germans Trias i Pujol, Badalona, Barcelona, Spain; Hospital Universitari General de Catalunya, Sant Cugat del Valles, Barcelona, Spain; Bellvitge Biomedical Research Institute (IDIBELL), L'Hospitalet del Llobregat, Barcelona, Spain.

Background: Transcriptional factor CDX2 is a major regulator of gut development and involved in colorectal cancer (CRC) as a suppressor gene. Lack of expression of CDX2 has recently been described in $4.1 \%$ of CRC and identifies among Stage II and III CRC a subgroup with worse prognosis. Herein, we analyze CDX2 immunohistochemical (IHC) expression and CDX2 methylation in different series of CRC to correlate the results with molecular profiles and prognosis.

Design: A TMA from 36 FFPE MSI-H MLH1(-)/Braf-mut CRC was analyzed by IHC with anti-CDX2 mouse monoclonal antibody (Dako Agilent Technologies). Additionally, epigenetic analysis of $123 \mathrm{CRC}$ was performed using Infinium 450K beadchip (Illumina). CDX2 was considered as methylated when average $\beta$-value in $\mathrm{CpG}$ island of promoter zone was over $50 \%$. In $14 \mathrm{CRC}$ both IHC and epigenetic analysis was performed. Clinical, molecular, and histopathologic parameters were established and statistically correlated.

Results: Ten of 36 (28\%) MLH1(-)/Braf-mut cases were CDX2(-). Lack of CDX2 expression correlated with CDX2 methylation, despite some CDX2 methylated cases retain CDX2 stain (12 IHC(+)/9 CDX2-Met). Regarding methylation, 55\% were CDX2 methylated, being $41 \%$ in MSS and $83 \%$ in MSI-H samples. CDX2 methylation was more frequent in MSI-H and mesenquimal-like phenotype groups (5/6 cases). Despite there was no correlation with any histopathologic parameter analyzed, in MSS group, p53-wt tumors showed higher methylation levels than those with p53-mut (U MannWhitney test; $p=0.008$ ). We observed a statistical significant association between CDX2 methylation with shorter relapse free survival in a set of patients of patients of whole cohort with clinical survival data available $(\mathrm{N}=44$; $\log$ Rank $\mathrm{P}=0.02)$.

Conclusions: Although in our set lack of $\mathrm{CDX} 2$ expression corresponds to CDX2 methylated samples, this mechanism is also related to cases with expression of CDX2. Our results suggest an enrichment of CDX2 silencing not only in MSI-H and mesenquimal-like cases, but also in MSS p53-wt tumors. Despite CDX2 methylation could be relevant as a prognostic factor in CRC, further studies are necessary to elucidate the relationship with protein expression by $\mathrm{IHC}$ and, consequently, its clinical impact. 
758

Characterization of Lgr5 Expression in Colorectal Neuroendocrine Tumor

Tomoyuki Nakajima, Takeshi Uehara, Yasuhiro Maruyama, Yukihiro Kobayashi, Hiroyoshi Ota, Takayuki Honda. Shinshu University School of Medicine, Matsumoto, Japan.

Background: Leucine-rich repeat-containing G-protein-coupled receptor $5(\operatorname{Lgr} 5)$ is a putative intestinal stem cell marker. $\operatorname{Lgr} 5$ is also expressed in various tumors. In this study, we have investigated $L g r 5$ expression in colorectal neuroendocrine tumor (NET) samples, and analyzed associated pathological characteristics.

Design: We evaluated the clinicopathological features of 8 NET grade 1 (G1), 4 NET grade 2 (G2), and 8 NET grade 3 (G3; also termed neuroendocrine carcinoma (NEC)) cases. We also measured $\operatorname{Lgr} 5$ expression using RNAscope ${ }^{\circledR}$, a newly developed RNA in situ hybridization technique, and a tissue microarray of NET samples. Lgr 5 staining in individual tumor cells was scored semi-quantitatively using an H-score scale. Ki-67, $\beta$-catenin, and synaptophysin were also detected in all cases by immunohistochemistry. We also performed a combination of Lgr 5 RNA in situ hybridization and synaptophysin immunohistochemistry.

Results: All cases contained tumor cells with some $\operatorname{Lgr} 5$-positive signals. The $\operatorname{Lgr} 5$ H-score was significantly higher in NET G3 (NEC) samples compared with those of NET G1 and G2. Both Lgr5-positive and synaptophysin-positive cells were observed in most cases. In all cases, $\operatorname{Lgr} 5 \mathrm{H}$-scores showed a positive correlation with nuclear $\beta$-catenin expression ( $r=0.6662, p=0.0013)$. There was no correlation between $\operatorname{Lgr} 5$ $\mathrm{H}$-score and Ki-67 expression. In the NET G1 and NET G2 groups, there was also no correlation between $\operatorname{Lgr} 5 \mathrm{H}$-score and Ki-67 expression, or between $\operatorname{Lgr} 5 \mathrm{H}$-score and $\beta$-catenin expression. In NET G3 (NEC) there was a strong positive correlation between $\operatorname{Lgr} 5 \mathrm{H}$-score and $\beta$-catenin expression $(r=0.8333, p=0.0102)$.

Conclusions: Lgr5 expression might be affected by $\beta$-catenin expression in NET and especially in NET G3 (NEC) lesions. The presence of Lgr5 and synaptophysin doublepositive cells suggests that Lgr5-positive cells may be also stem cells in NEC, as is the case in colon adenocarcinoma. A further study with a larger number of NEC cases is warranted to confirm these findings.

759 Mass Spectrometric Analysis of Gastrointestinal Amyloidomas Megan O Nakashima, Claudiu V Cotta. Cleveland Clinic, Cleveland, $\mathrm{OH}$.

Background: Amyloid is an incidental finding in samples originating from the gastrointestinal (GI) tract and its characterization by immunohistochemical (IHC) techniques is notoriously difficult. At least 31 proteins are known to have amyloidogenic potential, and reliable antibodies for the identification of many of these are currently lacking. While IHC is successful in characterizing up to $90 \%$ of cardiac amyloid deposits, in the GI tract IHC is successful in less than $40 \%$ of cases. Therefore, with the exception of one study on gastric amyloidomas, there are limited reliable data on the nature of incidental amyloidomas diagnosed in the GI tract. To fill this gap we employed mass spectrometry to analyze amyloid deposits from GI samples.

Design: 20 cases of amyloidomas from the GI tracts of adults were identified. Amyloid material was microdissected from the paraffin-embedded tissue, proteins extracted and digested with bovine trypsin, and analyzed by liquid chromatography tandem mass spectrometry (LC-MS/MS). The $\mathrm{m} / \mathrm{z}$ spectra were compared against existing databases (Swissprot) by a specialized search engine (Mascot), leading to identification of proteins by their peptide spectrum match (PSM). Serum P component, apolipoprotein AI, apolipoprotein AIV and apolipoprotein E identification confirmed the diagnosis of amyloid. Known amyloidogenic proteins were ranked according to PSM. Proteins with the highest PSM were interpreted as the main amyloidogenic components.

Results: The most frequent location of incidental gastrointestinal amyloidomas is the stomach (7/20 cases), followed by small intestine (6/20), liver (6/20) and colon (3/20). The most frequent amyloidogenic proteins are lambda immunoglobulin light chains (AL Lambda, 12/20), followed by kappa light chains (AL Kappa, 4/20), combination of both light chains (AL Both, 2/20), serum amyloid A (AA, 1/20) and transthyretin (ATTR, 1/20).

\begin{tabular}{|c|c|c|c|c|c|}
\hline Organ & AL Lambda & AL Kappa & AL Both & AA & ATTR \\
\hline Stomach & 2 & 0 & 1 & 0 & 0 \\
\hline Small Intestine & 3 & 1 & 0 & 0 & 1 \\
\hline Colon & 4 & 2 & 1 & 0 & 0 \\
\hline Liver & 3 & 1 & 0 & 1 & 0 \\
\hline
\end{tabular}

In both non-AL cases the GI amyloid deposits were the first manifestation of disease. Conclusions: Amyloidomas occur in most segments of the GI tract and in the liver. As in other locations, immunoglobulin light chains, in particular lambda, are the most common amyloidogenic proteins. While less frequent, other types of amyloidosis can also involve the GI tract, as demonstrated by the cases of ATTR and AA, and may be manifestations of systemic disease.

$760 \quad$ Using Pathology Reports to Assess the Endoscopist's Catch and Pathologist's Call in a Series of 11,456 Large Bowel Polyp Specimens with a Focus on Serrated Polyps

Asghar Naqvi, Jennifer M Dmetrichuk, Michael Bonert. McMaster University, Hamilton, ON, Canada.

Background: Variations in polyp acquisition and interpretation are not routinely assessed using clinical data, and may give insight into modifiable factors that influence early detection and prevention of colorectal cancer (CRC). Observational data is commonly used in process management/optimisation in other sectors.

Design: Surgical pathology reports for a 3 year period, in an academic centre, were data-mined using a custom computer program that extracted all large bowel polyp specimens and their diagnostic information using keyword searches, a fuzzy matching algorithm, and rule-based pruning. The categorizations by the computer were compared to pathologist reads for a random subset of reports to assess accuracy.

Results: Computer based categorization was successful in $99 \%$ of cases. An audit of 200 cases revealed that the algorithm accurately categorized $>99 \%$ of cases. The pathologists' and endoscopists' Sessile Serrated adenoma (SSA) rates ave/median/ stdev/max/min were: $2.6 \%, 1.9 \%, 2.6 \%, 7.7 \%, 0.0 \%$, and $1.8 \%, 1.8 \%, 0.7 \%, 3.3 \%$, $0.4 \%$ respectively. The pathologists' and endoscopists' Hyperplastic polyp (HP) rates by comparison were: $25.8 \%, 25.6 \%, 6.4 \%, 36.4 \%, 16.9 \%$ and $26.3 \%, 26.5 \%, 2.4 \%$, $30.7 \%, 22.4 \%$ respectively. The left and right large bowel HP rates ave $/$ median $/ \mathrm{stdev} /$ $\max / \mathrm{min}$ for pathologists were $20.3 \%, 20.4 \%, 4.6 \%, 27.3 \%, 13.1 \%$ and $2.4 \%, 2.9 \%$, $1.3 \%, 4.0 \%, 0.0 \%$ respectively.

Pathologists could be separated into two groups based on the SSA rate. Pathologists with more years in practice tended to (i) have lower SSA rates, (ii) never call SSA on the left side of the large bowel, and (iii) called more HPs on the right side.

Conclusions: The stdev is higher among pathologists for HP and SSA than for endoscopists. This implies that the variation is predominantly due to the interpretation, and reducing it would likely improve risk stratification in colorectal cancer screening and help optimise patient management.

Observational data stratified by pathologist can give insight into the application of knowledge and how practices evolve. The large differences in the SSA suggests a lack of uniformity in the diagnostic criteria/diagnostic threshold. These may be addressed by (1) developing in house consensus criteria, (2) subspeciality review of cases by outlier pathologists, and (3) on-going SSA rate auditing with comparison to literature value rates.

\section{Gastrointestinal and Pancreatic Findings in Patients with} McCune-Albright Syndrome

Michaël Noë, Laura D Wood, Wenzel Hackeng, Lodewijk AA Brosens, Feriyl Bhaijee, Marija Debeljak, Jun Yu, Alison Boyce, Cemre Robinson, James R Eshleman, Ralph H Hruban, Michael G Goggins, Michael T Collins, Anne Marie Lennon, Elizabeth A Montgomery. Johns Hopkins University, Baltimore, MD; University Medical Center Utrecht, Utrecht, Netherlands; National Institutes of Health, Bethesda, MD.

Background: McCune-Albright Syndrome (MAS) is a rare sporadic syndrome caused by somatic (post-zygotic), activating mutations in the G protein- $\alpha$ stimulatory subunit GNAS. Patients have a clinical triad of skeletal, endocrine and dermatological abnormalities including fibrous dysplasia of bone, precocious puberty, and café-au-lait spots. The same GNAS mutation in codon 201 has been reported in several gastrointestinal, pancreatic and other tumor types.

Design: We report the findings from upper endoscopic and imaging examination of 7 patients with MAS and pancreatic findings in 2. A variety of polyps and lesions was found throughout the esophagus, stomach, small intestine and pancreas. Lesions were biopsied/resected and submitted for histopathological evaluation and GNAS mutation analysis by pyrosequencing the FFPE tissues.

Results: All seven patients had heterotopic gastric mucosa (polypoid mucosa with oxyntic and/or foveolar type epithelium, outside the stomach), with GNAS mutations in $12 / 17$ lesions. Gastric hyperplastic-type polyps were present in 5/7 patients ( $2 / 5$ GNAS mutated), while fundic gland polyps arose in $2 / 7$ patients (both $G N A S$ wild-type). One patient had a Peutz Jeghers-like polyp (GNAS wild-type) of the ampulla of Vater, but with less smooth muscle proliferation and more inflammation. One patient had intestinal metaplasia at the gastroesophageal junction. All patients had EUS findings, suggestive of intraductal papillary mucinous neoplasm (IPMN), but only 2 patients met criteria for surgical intervention. Resection confirmed the IPMN diagnosis (2/2 GNAS mutated) in both patients and revealed a small duodenal proliferation of neuroendocrine cells (GNAS wild-type), bordering on a well-differentiated neuroendocrine tumor, in one patients. Two patients had high-grade dysplasia (HGD): in an IPMN and in a gastric polyp. In all but 1 analyzed sample, the adjacent normal tissue was GNAS wild-type. Conclusions: We described the pathological spectrum of gastrointestinal and pancreatic lesions in patients with MAS. We found enrichment for lesions known to have GNAS mutations (IPMNs, gastric heterotopia, gastric adenomas with oxyntic differentiation). Finding HGD in 2 patients raises the possibility of an increased risk for malignant transformation.

\section{A Study of Appendiceal Crypt Cell Adenocarcinoma (So-Called} Goblet Cell Carcinoid and Its Related Adenocarcinoma)

Daisuke Nonaka, George Papaxoinis, Angela Lamarca, Paul Fulford, Juan Valle, Bipasha Chakrabarty. The Christie Hospital, Manchester, United Kingdom; The University of Manchester, Manchester, United Kingdom.

Background: Goblet cell carcinoids (GCCs) of appendix are rare tumors, characterized by a carcinoid-like organoid growth pattern of nests and glands, and predominant cellular component is generally mucinous cells with goblet/signet-ring morphology. Despite the term carcinoid, endocrine features are inconspicuous, and its behavior is distinct from carcinoid. Its high grade counterpart is designated as adenocarcinoma ex goblet cell carcinoid (AEGCC). We conducted a retrospective study of 105 GCCs/AEGCCs to find prognostic values of a variety of clinico-pathologic features.

Design: The tumors were subclassified as low grade (LG), equivalent to classic type (Tang A), and high grade (HG), corresponding to Tang B\&C, and a proportion (\%) of $\mathrm{LG}$ and $\mathrm{HG}$ were documented in each tumor. Correlations between survival and the following were investigated: age, gender, stage, size, perineural, vascular and lymphovascular invasion, nuclear grade (low vs. high), histological HG component $(\%)$, mucous cell (\%), neuroendocrine (\%), extracellular mucin (\%), type of surgery, resection margin, and chemotherapy.

Results: Age at diagnosis ranged 25-79years (median, 54), with M:F=1:0.94. 35 tumors were pure LG while the remainder contained variable HG component ranging $1-95 \%$. 
Neuroendocrine component ranged $0-90 \%$ (median 5) while mucous cell componen ranged $2-100 \%$ (median 70). Follow-up ranged 3-277 months (median 56). Median overall survival (OS) was 67 months. By univariate analysis, size, stage, HG component (increasing proportion), nuclear grade, surgery and chemotherapy correlated with disease-related OS, and by multivariate analysis, stage $(\mathrm{p}=0.010)$ and $\mathrm{HG}$ component $(\mathrm{p}=0.041)$ correlated with OS. There was significant difference in survival when the cases were grouped in HG component $<40 \%, 40-89$ and $\leq 90 \%$.

Conclusions: Our results indicate that both staging and proportion of $\mathrm{HG}$ componen provide important prognostic information, and \% of HG component should be documented in pathology report. Neuroendocrine component was generally insignificant, therefore, designation of adenocarcinoma such as crypt cell adenocarcinoma would be appropriate for both GCC/AEGCC. In this simple histologic 2-tier grading, the proportion of $\mathrm{LG}$ and $\mathrm{HG}$ component seems to reflect degree of aggressiveness. Such a method might be more easily adoptable.

\section{Detection of Anaplastic Lymphoma Kinase (ALK) Rearrangement} in Colorectal Cancer: An Immunohistochemical Study of 128 Cases

Sahar Nozad, Sungeun Kim, Hwajeong Lee, Christine E Sheehan, David M Jones, Jeffrey Ross. Albany Med Col, Albany, NY.

Background: Discovery of anaplastic lymphoma kinase (ALK) gene rearrangement in a subset of lung adenocarcinomas has led to a standard of practice management with targeted therapy. Immunohistochemistry (IHC) has been approved as a screening tool to identify ALK rearrangement in lung cancer. ALK rearrangement appears to be very rare in colorectal cancer (CRC). We tested the utility of ALK IHC as a screening tool to detect ALK rearrangement in CRCs of variable molecular pathways.

Design: Archived pathology materials from 71 randomly selected CRCs, 19 mismatch repair (MMR) deficient CRCs, 38 sessile serrated polyp (SSP)-associated lesions ( 25 SSP, 3 SSP with low or high grade dysplasia, 10 SSP associated CRCs) were retrieved and reviewed. An index CRC case with ALK rearrangement confirmed by advanced molecular technique and fluorescent in situ hybridization (FISH) served as a known positive. Representative sections from paraffin-embedded formalin-fixed tissue blocks were subject to immunohistochemical stain for rabbit monoclonal anti-ALK (D5F3; Cell Signaling/Ventana). Ganglion cells served as internal positive controls. Cytoplasmic immunoreactivity in the tumor/polyp was evaluated. Selected cases were subject to repeat IHC with an increased incubation time to confirm protocol sensitivity.

Results: Twelve (9\%) CRCs showed focal weak cytoplasmic staining for ALK, comparable to the intensity and extent of ALK immunoreactivity in the index case as follows: 5 (7\%) random, 5 (26\%) MMR deficient, and 2 (20\%) SSP-associated /MMR proficient CRCs. The index case was MMR proficient, right sided colon cancer with marked intratumoral morphologic heterogeneity. The intensity or extent of ALK protein expression did not differ with increased incubation time.

Conclusions: A subset of CRCs are ALK positive by IHC, similar to a known ALK rearranged CRC. ALK IHC positivity is more frequent in MMR deficient CRC, suggestive of a role of high mutational load in ALK expression. IHC may potentially be used as a screening tool for detection of ALK rearrangement in CRCs. Comprehensive genomic profiling of the ALK IHC-positive cases in this study set is warranted.

764 Co-Expression of HER Family Relates to Progression and Lymph Node Metastases in Human Colorectal Cancer

Hiroyuki Nozaka, Ai Igarashi, Miku Togashi, Sayaka Kurosawa, Yayoi Takahashi, Noriyuki Uesugi, Kazuyuki Ishida, Tamotsu Sugai. Hirosaki University, Hirosaki, Aomori, Japan; Iwate medical university, Morioka, Iwate, Japan.

Background: The carcinogenesis of colon and rectum is caused by accumulation of genetic alternation or mutation, and it is reported that $A P C, p 53, D C C$ and $K$-ras gene mutations or loss are significant predictive factors. HER family is composed of four members, and Epidermal Growth Factor Receptor (EGFR) is a member of HER family. EGFR is expressed in the case of $60-80 \%$ of colorectal cancer, and it is reported that the expression of EGFR is strongly involved in the invasion and metastasis. Meanwhile, it is known that HER family member formed dimer structure with other HER family member, but a few reports shows co-expression of HER family and clinicopathological significance in human colorectal cancer. In this study, we examined co-expression of HER family mRNA and protein in human colorectal cancer, and evaluated significance as the clinicopathological biomarker.

Design: Tumors for this study were collected from 60 patients diagnosed with primary advanced colorectal cancer. After the surgery extraction, we collected a part of both normal and tumor tissues from fresh colon or rectum. The half of tissue was used for extraction of total RNA with TRIzol reagent, and the left-behind tissue was fixed in the $10 \%$ formalin and embedded in the paraffin (FFPE). EGFR, HER2/3/4 protein was detected by immunohistochemistry on FFPE tissue sections. EGFR, HER2/3/4 mRNA expression were analyzed by qRT-PCR. Statistical analysis for clinicopathological evaluation was carried out by SPSS21.

Results: 1) IHC analysis : The co-expression of EGFR+/HER2+ was showed only $2 \%$ of all cases. EGFR+/HER3+ and EGFR-/HER3+ was showed $51 \%$ and $24 \%$ respectively. EGFR+/HER4+ and EGFR-/HER4+ was showed $40 \%$ and $27 \%$ respectively. HER3+ was statistically significant between EGFR+ and -. EGFR+ showed significant difference in the lymph node, tumor size and invasion. EGFR+/HER3+ showed difference in the lymph node metastasis.

2) qRT-PCR analysis : $E G F R+/ H E R 3+$ and $E G F R+/$ HER3- was showed $93 \%$ and $7 \%$ respectively, HER3 expression rate of $E G F R+$ cases was higher compared to the $E G F R$ - cases.HER 3 expression related to tumor size and invasion significantly. Conclusions: It was suggested that EGFR/HER3 associated with the progression or lymph node metastasis of the colorectal cancer. It seems that HER3 has the potential of a new molecular target in colorectal cancer therapy. However, this study does not disclose the relevance of KRAS or PI3K mutations. In our future work, we will study the relationship of cancer-related gene mutations or the prognosis.
$765 \quad$ Chromogenic In Situ Hybridization (CISH) Based Tracking of Donor Derived Lymphocytes Augments the Histologic Evaluation of Human Acute Graft versus Host Disease (GVHD)

Kwadwo Oduro, Kshitij S Arora, Vikram Deshpande. Massachusetts General Hospital, Boston, MA.

Background: Acute GVHD (aGVHD), a significant complication of allogeneic hematopoietic stem cell transplantation (BMT), is thought to be driven by allo-reactive donor derived lymphocytes. However, direct histological demonstration of these cells in tissue is lacking. The histologic diagnosis of aGVHD in the gastrointestinal tract is based on a quantitative assessment of apoptotic epithelial cells. This finding is not specific and is frequently subtle. There is the need for techniques to improve on the pathologic diagnosis of aGVHD and to shed light on the mechanism of this phenomenon. Design: We enumerated the number of crypt apoptoses in duodenal and colonic biopsies $(\mathrm{N}=18)$, obtained from female to male BMT patients, with a clinical question of aGVHD. Using a CISH probe for the female specific transcript, XIST (X-inactive specific transcript), we scored for the presence of intraepithelial donor derived $(X I S T+)$ mononuclear cells (IE-XIST), and quantified the percentage of XIST+ lamina propia mononuclear cells (LP-XIST\%). Infectious etiologies were excluded. We also documented donor chimerism in the peripheral blood (PB) around the time of the biopsy. Results: LP-XIST\% positively correlated with the number of apoptosis $\left(\mathrm{R}^{2}=0.32\right.$, $\mathrm{p}=0.014$ ). LP-XIST $\%$ also showed a significant association with IE-XIST (Mean LPXIST: $35 \pm 8 \%$ in the IE-XIST present group versus Mean LP-XIST:16 $\pm 4 \%$ in the IE-XIST absent group, $\mathrm{p}=0.032$ ). There was no association between LP-XIST $\%$ and the time interval from BMT to biopsy. LP-XIST (range 0-60\%, mean 24\%) was also significantly lower than PB total chimerism (range 90-100\%, mean 98\%, $\mathrm{p}=1 \times 10^{-17}$ ) or PB CD3+ T lymphocyte chimerism (range $80-100 \%$, mean $95 \%, \mathrm{p}=5 \times 10^{-16}$ ). Using LP-XIST threshold of $20 \%$, derived from linear regression analysis, the 4 cases with both LP-XIST $\%>20 \%$ and positive IE-XIST all required immunosuppressive therapy for clinical control and most $(75 \%)$ had histologic features of aGVHD in other organs. Conversely, of the cases with LP-XIST $<20 \%$ and no IE-XIST $(\mathrm{N}=6), 3$ cases $(50 \%)$ clinically resolved without anti-GVHD therapy. All 6 cases in the cohort that clinically resolved without aGVHD treatment lacked IE-XIST.

Conclusions: Host intestinal lymphocytes persist after BMT such that allo-reactive donor derived cells form a minority of lamina propia cells in most cases of aGVHD. In female to male BMT, we propose that CISH for XIST could be used as an adjunctive tool in the histologic evaluation of suspected aGVHD, specifically, in biopsies showing borderline increase in apoptotic cells.

766 in the Transplant Population

Nkechi Okonkwo, William Twaddell. University of Maryland, Baltimore, MD.

Background: The development of colorectal cancer (CRC) proceeds via at least three molecular pathways including chromosomal instability, microsatellite instability (MSI), and $\mathrm{CpG}$ island methylator phenotype. MSI-associated CRC has distinct histologic features as well as a slightly better prognosis when compared to tumors without MSI. MSI is a hypermutable phenotype which is implicated in different types of cancers and is caused by deficiencies in the expression of mismatch repair (MMR) proteins. In humans, the MMR proteins associated with MSI include MLH1, MSH2, MSH6, and PMS2. A lack of these proteins leaves the DNA vulnerable to mutations that can subsequently lead to cancer.

Transplant recipients have an increased risk of cancer, including CRC, when compared to the general population due to immunosuppression. MSI-associated CRC is a tumorigenesis pathway which may be inherited, but more often occurs sporadically, and carries recently described implications for therapy. In the general population, the frequency of MSI-associated CRC is approximately $12 \%-17 \%$; however, the frequency within the transplant population has not been described. By understanding the mechanisms which CRC develops, potential therapies can be optimized for this patient population that carries an increased risk.

Design: Electronic medical records were searched to identify patients with a history of both organ transplantation and development of CRC after transplantation. MMR protein expression in these individuals was evaluated using immunohistochemical staining for MMR proteins - MLH1, MLH2, PMS1, and PMS2. We evaluated the frequency of MMR protein expression loss in CRC of patients with a history of organ transplantation and compared the results with the control group of CRC patients without a history of transplantation.

Results: Among 14 cases of CRC, 7 had a history of development of colorectal cancer after organ transplantation. 5 of 7 cases had complete or partial loss of MMR protein expression compared with no loss of expression in the controls $(71.4 \%$ vs $0 \%, P=$ 0.005 , Fisher's exact test). Of the cases with loss of MMR protein expression, $100 \%$ ( 5 of 5 cases) had loss of MLH1 and PMS2 expression and one additionally had a loss of MSH2 and MSH6 expression.

Conclusions: $\mathrm{CRC}$ in the setting of organ transplantation showed an increased loss of MSI-associated proteins, specifically MLH1 and PMS2, when compared to the controls. This may carry implications for screening and treatment of patients following transplantation. 
767 Histogical Characteristics Correlated with MSI Status in Colorectal Carcinomas in National Hospital Abuja, Nigeria

Friday Olah, Fatimah B Abdulkareem, Paul G Jibrin, Olusegun S Ojo, Said M Amin, Emmanuel E Oguntebi, Aisha NAdowanka, Henry Ewunonu. National Hospital, Abuja, FCT, Nigeria; Lagos University Teaching Hospital, Idi Araba, Lagos, Nigeria; Obafemi Awolowo University Teaching Hospital, Ile Ife, Osun, Nigeria.

Background: Tumours with microsatellite instability(MSI) occur more frequently in younger patients, located proximal to the splenic flexure and exhibit exophytic growth, poor differentiation, extracellular mucin production, and a Crohn's-like lymphocytic reaction. Such tumours have an improved survival rate relative to microsatellite-stable (MSS) tumours of similar stage.Recognition of the microsatellite phenotype is important in the management of patients with CRC. Correlation of the histological types and molecular testing of $\mathrm{CRC}$, using MMR proteins, is the thrust of this work.

Design: We reviewed the histological characteristics of 175 CRC cases spanning a 10year period. We then subjected them to IHC study for their MSI status and compared the two parameters.

Results: Overall, 140 out of 175 samples were tested mismatch repair (MMR) protein loss using standard IHC. Fourty five (45) patients $(32.1 \%)$ had high frequency Microsatellite instability (MSI-H) tumours, 75 patients $(53.6 \%)$ had microsatellite stable (MSS) tumours and $20(14.3 \%)$ had low frequency (MSI-L) tumours. There were no recognizable patterns between MSI status and age, gender or histological type of the tumour ( $\mathrm{p}$ values of $0.904,0.999$ and 0.302 respectively). A right sided location and poor differentiation were observed in $51.0 \%$ and $66.7 \%$ of MSI-H tumours, respectively, versus $49.0 \%$ and $33.3 \%$ of MSS tumours, respectively ( $p$ values of 0.013 and 0.00 , respectively). Exophytic growth pattern was more common for MSI-H tumours. The annular tumours were more likely to be MSS ( $p$ value .023). The strongest associations were found between MSI status and residual adenomatous tissue, worse tumour grade, Crohn-like feature and pathological staging with $\mathrm{p}$ values $<0.001$. Although MSI-H colorectal cancers were diagnosed at a significantly greater depth of tumour invasion, these tumours had a significantly lower overall pathological stage than cancers with microsatellite stability.

Conclusions: High rate of MSI-H tumours were seen even though. Histological features correlated with MSI status. Grade and stage rather than histological type should rouse suspicion of MSI-H tumours and familial cancer syndromes. Residual adenomatous tissue and MSI status correlated strongly in our study emphasising the need to increase screening activities especially in at-risk patients and family members.

Histologic Features of Mismatch Repair (MMR) Deficiency Do Not Reliably Predict Immunohistochemical Detection of MMR Deficiency in Colorectal Carcinoma: The Results of a 5-Year Prospective Evaluation Dane Olevian, Reetesh Pai. University of Pittsburgh Medical Center, Pittsburgh, PA. Background: Identification of mismatch repair (MMR) protein deficiency (MMRD) in colorectal carcinoma (CRC) is advocated to screen for Lynch syndrome and is an important prognostic and predictive biomarker. The College of American Pathologists (CAP) protocol for CRC advocates the reporting of histologic features of MMRD. However, the reliability of histologic features in predicting MMRD has not been fully evaluated.

Design: 942 consecutive CRCs over a 5-yr period were assessed for histologic features of MMRD (tumor infiltrating lymphocytes, Crohn's like inflammation, mucinous/signet ring differentiation, and medullary differentiation). Histologic features of MMRD were reported as present (the presence of any histologic feature) or absent (the lack of all histologic features). All cases were prospectively evaluated for MMRD using MMR immunohistochemistry (IHC) for MLH1, MSH2, MSH6, and PMS2. The assessment of histologic features of MMRD was documented in the pathology report prior to and without knowledge of the MMR IHC results.

Results: Table 1 details the correlation of histologic features and IHC. Histologic features of MMRD had the following test characteristics for predicting MMRD by IHC: sensitivity $73 \%(66-80 \%)$, specificity $78 \%(75-81 \%)$, positive predictive value $38 \%$ $(32-44 \%)$, and negative predictive value $94 \%(92-96 \%)$. Importantly, the sensitivity of histologic features of MMRD in predicting MMRD by IHC was much lower in the left colon/rectum (56\%) compared to the right colon ( $78 \%$ ) (Table 2).

Table1: Histologic Features of MMRD and MMR Immunohistochemistry

\begin{tabular}{|l|c|c|}
\hline $\begin{array}{l}\text { Histologic Features of MMR } \\
\text { Deficiency }\end{array}$ & $\begin{array}{c}\text { MMR Proficient by } \\
\text { IHC (\%) }\end{array}$ & $\begin{array}{c}\text { MMR Deficient by } \\
\text { IHC (\%) }\end{array}$ \\
\hline No of Cases & 799 & 143 \\
\hline Present & $174(22)$ & $105(73)$ \\
\hline Absent & $625(78)$ & $38(27)$ \\
\hline
\end{tabular}

Table 2: Sensitivity and Specificity of Histologic Features of MMRD in Predicting MMR Immunohistochemistry

\begin{tabular}{|l|c|c|}
\hline Tumor Site in the Colon/Rectum & Sensitivity (\%) $(95 \% \mathrm{CI})$ & Specificity (\%) $(95 \% \mathrm{CI})$ \\
\hline All Sites & $73(66-80)$ & $78(75-81)$ \\
\hline Right Colon & $78(69-85)$ & $69(63-74)$ \\
\hline Left Colon / Rectum & $56(38-74)$ & $83(80-86)$ \\
\hline
\end{tabular}

Conclusions: Our prospective evaluation demonstrates that histologic features of MMRD do not reliably predict MMRD by IHC. This is particularly evident in tumors of the left colon/rectum. The CAP protocol should be modified to advocate for universal screening of all CRCs for MMRD given the lack of sensitivity and specificity of histologic features in predicting MMRD by IHC.
Somatic Variant Profiles of TP53 Mutant Colorectal Carcinomas Andrea Olofson, John He, Jason D Peterson, Sophie J Deharvengt, Francine B de Abreu, Xiaoying Liu, Gregory J Tsongalis. Dartmouth-Hitchcock Medical Center, Lebanon, NH. Background: According to the Cancer Genome Atlas Network, the most frequently mutated genes in colorectal carcinoma are; APC (81\%), TP53 (60\%), KRAS $(43 \%)$, TTN (31\%), PIK3CA (18\%), FBXW7 (11\%), SMAD4 (10\%), NRAS (9\%), and TCF7L2 $(9 \%)$. Mutations in the TP53 gene are of particular interest as they are identified in over half of all human cancers. The protein product of this gene, p53, functions as an important DNA-binding transcription factor that induces apoptosis in the event of cellular stress. The loss of wildtype $\mathrm{p} 53$ expression allows tumor cells to avoid apoptosis and develop additional, possibly more aggressive phenotypes, through subsequent mutations. Here, we sought to characterize the mutational composition of colorectal carcinoma tumors harboring TP53 mutations and identify any potential correlation with clinical presentation.

Design: DNA was extracted from formalin-fixed, paraffin-embedded (FFPE) tissues and sequenced using $318 \mathrm{v} 2$ chips on the Ion Torrent $\mathrm{PGM}^{\mathrm{TM}}$ System. Sequencing was performed using a 50 gene cancer hotspot panel (AmpliSeq v2, Life Technologies) that included the TP53, ATM, BRAF, CDKN2A, ERBB2, FBXW7, FGFR3, JAK3, KRAS, NRAS, MET, PIK3CA, PTEN, SMAD4, STK11, APC and 34 additional cancer related genes. Variants were identified using the Variant Caller Plugin (v.4.0), and annotation and functional predictions were performed using Golden Helix SVS (v.8.3.4).

Results: A total of 132 colorectal carcinoma tumors containing TP53 mutations were identified. Of these, the most common TP53 mutations occurred in codons 248 (R248Q $6 \%, \mathrm{R} 248 \mathrm{~W} 2 \%$ ) and 282 (R282W 5\%). The majority of these tumors contained at least one additional mutation $(69 \%)$. The most common additional mutations were seen in KRAS (27\%), BRAF (20\%), and PIK3CA (10\%). TP53 mutated tumors occurred more frequently in the rectum $(34 \%)$, sigmoid colon $(16 \%)$, and cecum $(14 \%)$. No correlations were identified between the number of mutations or combination thereof in relationship to tumor grade or stage at the time of diagnosis.

Conclusions: Our study indicates that colorectal carcinomas harboring TP53 mutations possess mutational profiles incongruent with colorectal carcinomas as a whole. Most notably are the complete lack of $A P C$ mutations and an increase in proportion of $B R A F$ mutations. Given the high frequency of $A P C$ mutations within colorectal carcinoma, the absence of these mutations within our subset of TP53 mutated tumors suggest that these mutations may be mutually exclusive of one another. Further investigation into the physiological significance of this finding is warranted.

$770 \quad$ Clinical Impact of Tumor Infiltrating Lymphocytes and Neutrophils in Advanced Gastric Cancer

Do Youn Park, Joo Yeun Kim, Young Keum Kim, So Jeong Lee. Pusan National University Hospital and Pusan National, Busan, Republic of Korea; Haeundae Paik Hospital, University of Inje College of Medicine, Busan, Republic of Korea.

Background: Tumor immunology has been extensively investigated over the past decade. As widely known, the tumor infiltrating lymphocytes (TIL) has been associated with improved clinical outcome of cancer patients. T cells recognized cancer antigen and induce immune responses for the elimination of cancer cells. Although neutrophils are traditionally considered in the context of their antibacterial functions, it is becoming increasingly clear that tumor-associated neutrophils (TAN) play a major role in cancer biology.

Design: We enrolled 211 advanced gastric cancers underwent gastrectomy with LN dissection and count lymphocytes and neutrophils on one high power field $(\mathrm{x} 400)$ in the two areas that are tumor center (TC) and invasive margin (IM), refer to TC

lymphocytes (TCL), IM lymphocytes (IML), TC neutrophils (TCN) and IM neutrophils (IMN).

Results: They are divided into two groups, low or high, based on average cell number. According to statistical analysis, low TCL is significantly associated with old age (over $65)(\mathrm{p}=0.039)$, tumor location (higher in body) $(\mathrm{p}=0.037)$, tumor invasiveness

(depth of invasion) $(\mathrm{p}=0.004)$ and positive lymphovascular invasion $(\mathrm{p}=0.006)$. And IMN is associated with high microsatellite instability $(\mathrm{p}=0.011)$. As TCL increases, IML and TCN increase. The patients with a lower number of TCL showed a lower overall survival rate $(\mathrm{p}=0.049)$

Conclusions: We demonstrated that two populations of tumor infiltrating immune cells, lymphocytes and neutrophils in the two distinct areas (TC and IM). There is statistically significant correlation between these two immune cells. In particular, TCL is highly associated with cancer progression and outcome.

771 Metachronous and Synchronous Gastric Neoplasm After Endoscopic Treatment of Gastric Epithelial Dysplasia: Impact of Intestinal Metaplasia and Crypt Dysplasia of Background Mucosa

Won-young Park, Do Youn Park, Kyung Un Choi, Gi Young Huh, Chang Hun Lee, Ahrong Kim, Young Keum Kim, So Jeong Lee, Chung Su Hwang. Hanmaeum Changwon Hospital, Changwon, Gyeongsangnam-do, Republic of Korea; Pusan National University Hospital, Busan, Seo-Gu, Republic of Korea.

Background: Endoscopic resection has been regarded as safe and effective method to treat gastric epithelia dysplasia (GED). However, development of metachronous and synchronouslesions after treatment has become major concerns.

Design: We investigated clinicopathologic features of 124 endoscopic resections of GED lesions between January 2008 and December 2009 to investigate indicators of occurrence of synchronous or metachronous gastric lesion after treatment of GED, including degree of intestinal metaplasia (IM) and atrophy, type of IM, presence of gastritis cystic and crypt dysplasia in the adjacent mucosa. We divided 123 GED lesions into three groups: single lesion: solitary $(n=35)$, multiple GED $(n=52$, group 
with metachronous and synchronous occurrence of GED after treatment), multiple adenocarcinoma $(\mathrm{ADC})(\mathrm{n}=37$, group with metachronous and synchronous occurrence of adenocarcinoma after treatment).

Results: Multiple GED, ADC groups revealed increased age $(\mathrm{p}=0.036)$ and larger size $(\mathrm{p}<0.0001)$ compared to solitary group. Furthermore, marked intestinal metaplasia, atrophy in adjacent mucosa were more easily seen in multiple GED, ADC groups compared to solitary groups $(\mathrm{p}<0.0001)$. Interestingly, presence of incomplete type of IM $(\mathrm{p}=0.003)$ and crypt dysplasia $(\mathrm{p}<0.0001)$ was associated with multiple GED, ADC groups.

Conclusions: Our results suggested that clinicopathological features of background mucosa, such as intestinal metaplasia, atrophy, and crypt dysplasia, could be used as indicator of occurrence of metachronous and synchronous gastric neoplasm after endoscopic treatment of gastric epithelial dysplasia.

\section{$772 \quad$ Histologic Features in Colorectal Sessile-Shaped Malignant}

\section{Polyps with Nodal Metastasis}

Natalie Patel, Romulo Celli, Buqu Hu, Dhanpat Jain, Xuchen Zhang. Yale University, New Haven, CT.

Background: Malignant polyps are defined as adenocarcinoma limited to the submucosa (pT1). It is known that tumor differentiation, lymphovascular invasion (LVI), margin status, polyp shape and size are important risk factors indicating possible lymph node metastasis, which justifies a colectomy. However, in daily practice the size, margin and LVI are often unknown or difficult to assess in a piecemeal polypectomy from sessile-shaped adenomatous polyps (SP). We aimed to evaluate morphologic features in SPs, such as cribriform (CR) and micropapillary (MP) architecture and tumor budding (TB), to determine if these features portend an increase metastatic potential warranting a resection procedure.

Design: The pathology database was searched to identify T1, N1 or greater colorectal carcinomas (CRC) arising from SPs from 2005-2010. Malignant SPs and their corresponding resection specimens were reviewed and the age, gender, TB, grade, morphology, tumor site and TNM stage were recorded. Malignant SPs with N0 designation were used as the control group. Sensitivity (Sn) and specificity (Sp) were calculated for different morphologies and their combinations. A logistic regression model was used to calculate odds ratio for each morphology.

Results: 15 cases $(6 \mathrm{~F}: 9 \mathrm{M}$, average age 63$)$ of nodal positive and 16 cases $(9 \mathrm{~F}: 7 \mathrm{M}$ average age 67) of nodal negative SPs were identified. Of the nodal positive SPs: $87 \%$ were low grade, $80 \%$ were high TB (HTB). CR and HTB together was seen in $71 \%$ of cases and MP and HTB was seen together in $50 \%$. Sn and Sp were calculated for the two morphologies with and without high tumor budding (table 1). The sensitivities of MP and CR increased when HTB was included to $67 \%$ and $71 \%$ respectively. The triple combination of HTB, CR and MP increased the sensitivity to $100 \%$. HTB, CR, and MP increased the risk of nodal positivity, with odds ratio of $3.5,2.9$, and 2.2 respectively. Conclusions: Our data showed lymph node metastasis can be seen in SPs with both low and high grade CRCs in the absence of polyp size, margin status and LVI. HTB, MP and $\mathrm{CR}$ architectures are important features in determining metastatic potential. In addition, HTB with either CR or MP architecture increases the risk of nodal metastasis. Therefore, these features should be reported and a more aggressive procedure may be considered.

\begin{tabular}{|l|l|l|l|l|}
\hline & CR & MP & HTB & 2 out of 3 \\
\hline Nodal Pos. SPs & $47 \%$ & $40 \%$ & $80 \%$ & $73 \%$ \\
\hline Nodal Neg. SPs & $19 \%$ & $31 \%$ & $50 \%$ & $31 \%$ \\
\hline Sensitivity & $55 \%$ & $70 \%$ & $60 \%$ & $\begin{array}{l}\text { MP+HTB: } 67 \%, \text { CR+HTB: } 71 \%, \text { ALL } \\
3: 100 \%\end{array}$ \\
\hline Specificity & $62 \%$ & $55 \%$ & $72 \%$ & $\begin{array}{l}\text { MP+HTB: } 59 \%, \text { CR+HTB: } 55 \%, \text { ALL } \\
3: 57 \%\end{array}$ \\
\hline
\end{tabular}

\section{Incidental Splenic Findings in Pancreaticosplenic Resections}

\section{for Primary Pancreatic Disorders}

Nisha Patel, Andrew Evans, Paul G Rothberg, Raul S Gonzalez. University of Rochester Medical Center, Rochester, NY.

Background: En-bloc distal pancreatectomy with splenectomy is considered routine surgical management for benign and malignant distal pancreatic disorders. As emphasis is placed on evaluating the pancreatic lesion, splenic diagnoses may be overlooked, particularly if not reviewed by a hematopathologist.

Design: We reviewed 191 cases of distal pancreatectomy with splenectomy performed at our institution. All were originally signed out by a surgical pathologist, without hematopathologist involvement. Existing H\&E spleen sections from each case were blindly reviewed by a hematopathologist, and concordance with original diagnoses was analyzed. Specimens suspicious for involvement by lymphoid malignancy underwent PCR molecular analysis for immunoglobulin heavy and light chain ( $I G H$ and $I G K$ ) rearrangements on archived paraffin-embedded tissue.

Results: Most spleens $(167,87 \%)$ were initially diagnosed as grossly and microscopically normal; 157 (94\%) remained normal on review. Potentially significant findings that were not originally identified included foamy macrophage infiltration $(n=2)$, focal necrotizing granulomas $(\mathrm{n}=1)$, focal abscess $(\mathrm{n}=1)$, and morphology suspicious for incidental leukemia/lymphoma $(\mathrm{n}=4)$. In addition, a single case of chronic lymphocytic leukemia (CLL) from a patient with known history was confirmed. All 5 cases with some evidence or suspicion of leukemia/lymphoma were analyzed for B cell clonality. Molecular analysis showed definitive monoclonal gene rearrangement of both $I G H$ and $I G K$ in 2 cases ( $1 \%$ of total), including the known CLL. One other case showed only weak polyclonal signals for both, which might be due to a clonal rearrangement that was not detected, or might be due to technical factors such as DNA degradation. The last two cases gave polyclonal (i.e. normal) results. Nineteen spleens were initially diagnosed as abnormal, but four $(21 \%)$ were normal on review; overturned diagnoses included granulomas $(n=2)$, rupture $(n=1)$, and congestion $(n=1)$. In five cases, the spleen was sampled but not mentioned in the final microscopic report.

Conclusions: Incidental splenic findings are not uncommon in pancreatosplenectomy specimens, and even hematopoietic malignancies can be occasionally overlooked. Given the increasing trend toward subspecialty signout, it is likely that these cases are assigned to pathologists without hematopathology expertise. We recommend heightened awareness and careful observation of splenic findings in these specimens, accompanied by a low threshold for appropriate hematopathology consultation when in doubt.

$774 \quad$ Localized Colonic Ischemia Producing a Mass and Clinically Mimicking Colon Carcinoma: An Unrecognized Variant of Ischemic Colitis Smita Patel, Shenon Sethi, Shriram Jakate. Rush University Medical Center, Chicago, IL.

Background: Ischemic colitis (IC) is a common disorder and clinically diagnosed in susceptible population (generally older patients) with predisposition (compromised perfusion). The typical presenting symptom is rectal bleeding (with or without abdominal pain and diarrhea). Endoscopically, the affected colon is erythematous with variable ulcerations and involving vulnerable segments (left colon) and sparing the rectum. Microscopically, these areas show diagnostic ischemic hyalinization and crypt atrophy and drop-out. We found and reviewed cases of ischemic colitis that produce a localized tumor-like mass (LIC-TLM) and create a strong clinical and endoscopic suspicion of carcinoma rather than IC.

Design: From our hospital's databases, we selected all cases with the final diagnosis of IC from 2008-2016 (967 total cases). From these patients, we further selected cases that were initially endoscopically diagnosed as "colonic mass" and reviewed their clinical presentation, predisposition for ischemia, endoscopic findings, pre-biopsy clinical/ endoscopic diagnoses, histological findings, and follow-up.

Results: 7 of 967 cases of IC $(<1 \%)$ presented as a mass (LIC-TLM). The patients (6 females and 1 male, age range 49-84 years) presented with variable complaints such as abdominal pain (4), rectal bleed (3), incidental finding on screening (2), and nausea (1). Predisposing conditions included valvular heart disease (3), cardiac dysrhythmia (3), ischemic cardiomyopathy (1), and recent surgery (2). Endoscopically, all 7 cases showed an exophytic, friable, and non-stricturing colonic mass strongly suggestive of malignancy. 6/7 cases were located in the right colon. The biopsies showed characteristic features of IC and no evidence of dysplasia or carcinoma. 2/7 cases underwent a second biopsy and 1/7 a surgical excision with the persistent clinical suspicion of carcinoma, again showing IC histologically. After being managed as IC, none of the cases revealed a colon carcinoma during follow-up.

Conclusions: A rare variant, $<1 \%$ of all IC, exists that produces a localized tumor-like mass, endoscopically strongly masquerading as carcinoma (LIC-TLM). Such LIC-TLM variant also differs from conventional IC in the form of right sidedness and female predominance. However, predisposing factors are similar to conventional IC. Clinical and pathological recognition of this variant is necessary. Without awareness of this, there is a potential for unnecessary repeat biopsies and surgeries in manageable cases of IC.

\section{Metastatic Malignancies to the Upper Gastrointestinal Tract} Encountered on Endoscopic Biopsy: A Clinicopathologic Study

Vatsal Patel, Elham Nasri, Jesse L Kresak, Michael Feely. University of Florida, Gainesville, FL.

Background: Metastatic tumors to the upper gastrointestinal tract (UGIT) are seldom encountered but when they occur they may cause diagnostic challenges. The literature regarding this topic has largely centered on autopsy studies with little attention to the most frequently encountered specimen from the UGIT, the endoscopic biopsy. In this study, we sought to better define the clinicopathologic features of such cases.

Design: We identified and reviewed cases of metastatic lesions to the UGIT from our institutional archives that were encountered over a 17 year period. Material received in consultation was excluded due to lack of relevant clinical information. Histologic and clinical chart review was performed to evaluate the microscopic, endoscopic and clinical features of each case.

Results: Thirty-eight patients were identified as having metastatic malignancies involving the UGIT including $18(47 \%)$ females and $20(53 \%)$ males; Ages were 26-84 (median: 63 years). Twenty-four $(63 \%)$ cases involved the duodenum; 8 (21\%) the stomach; $3(8 \%)$ the ampulla; $2(5 \%)$ the gastroesophageal junction; and $1(3 \%)$ both the stomach and duodenum. Nine $(24 \%)$ cases represented metastatic breast carcinoma; 7 (18\%) colonic adenocarcinomas; $6(16 \%)$ malignant melanomas; $6(16 \%)$ renal cell carcinomas; and 1 (3\%) each from lung, esophageal, gallbladder, gastric and endometrial adenocarcinomas as well as cholangiocarcinoma, papillary serous carcinoma of the ovary and a testicular yolk sac tumor. Two $(5 \%)$ cases represented carcinomas of unknown primary. The most common metastatic tumor to involve the stomach was breast carcinoma ( 6 of 9 cases, $67 \%$ ) while the most common metastatic lesions to the duodenum were renal cell carcinoma and colonic adenocarcinoma ( 5 of 25 cases each, $20 \%$ ). Twenty-five (66\%) patients had previous or concurrent metastatic disease involving other organs while an additional $6(16 \%)$ had metastatic disease to only lymph nodes. In $4(10 \%)$ cases, UGIT metastasis was the primary presentation of malignancy. Of the remaining 34 patients, $16(47 \%)$ had no malignancy history noted on the accompanying specimen requisition form. Of the cases available for histologic review, 4 of 31 (13\%) were restricted to tumor involving lymphovascular spaces. Conclusions: UGIT metastases are quite varied depending on the location and are typically associated with metastatic disease elsewhere. Caution is advised when evaluating endoscopic biopsies as these lesions may be subtle and a prior history of malignancy may not be relayed to the pathologist. 
776 Immunohistochemical and Molecular Characterization of Dysplasia Subtypes in Ulcerative Colitis

Deepa T Patil, John R Goldblum, Robert D Odze. Cleveland Clinic, Cleveland, OH; Brigham and Women's Hospital, Boston, MA.

Background: The morphologic spectrum of dysplasia in IBD has recently expanded to include variants that are poorly characterized. Little is known about the biological features of these subtypes. The aim of our study was to assess a variety of immunohistochemical and molecular markers in different dysplasia subtypes of $\mathrm{UC}$, to determine their biologic significance, and investigate potential pathways of carcinogenesis.

Design: 30 dysplastic foci [15 low-grade (LGD), 15 high-grade (HGD)] from 23 UC patients $(8 \mathrm{M}, 15 \mathrm{~F})$ were reviewed and classified as: adenoma-like [elongated, pseudostratified nuclei $(n=15)]$, terminally differentiated $(n=4)$, serrated $(n=7)$, and hypermucinous ( $\mathrm{n}=4)$. All cases were stained for MUC2, MUC5AC, MUC6 and markers of colorectal carcinogenesis [p53, $\beta$-catenin (nuclear), annexin A10 (ANXA10), Maspin, BRAF (cytoplasmic), SOX 9 (nuclear)]. Staining in $>10 \%$ cells was considered positive. Results: The distribution of markers in 30 dysplastic foci was: MUC2-90\%, MUC5AC-0\%, MUC6-7\%, p53-48\%, $\beta$-catenin-30\%, ANXA10-17\%, BRAF-7\%, Maspin- $87 \%$, and SOX9-100\%. Compared to LGD, HGD showed frequent MUC6 ( $13 \%$ vs $0 \%), \beta$-catenin $(40 \%$ vs $20 \%)$, ANXA10 ( $27 \%$ vs $6 \%)$, and p 53 expression $(87 \%$ vs $7 \%)$; only $\mathrm{p} 53$ was significant $(\mathrm{p}<.001)$. After analyzing each subtype, all adenoma-like dysplasia were MUC2 positive; one case was diffusely MUC6 positive. All 4 hypermucinous dysplasias expressed MUC2, 2/4 expressed MUC6, and none expressed MUC5AC. The most commonly altered marker in adenoma-like dysplasia was p $53(33 \%)$, whereas terminally differentiated subtype showed equal p53 and $\beta$-catenin expression $(50 \%)$. Majority of hypermucinous dysplasia cases ( $75 \%$ ) showed aberrant p53. BRAF positivity was only seen in serrated dysplasia. Serrated dysplasia was most commonly associated with p $53(57 \%)$ and $\beta$-catenin (43\%) expression, while ANXA10 was only seen in $14 \%$ cases. While none of comparisons were statistically significant, ANXA10 was significantly more common in terminally differentiated compared to adenoma-like dysplasia ( $50 \%$ vs $7 \% ; \mathrm{p}=0.009)$. None of the markers showed any significant correlation with location or endoscopic appearance of dysplasia.

Conclusions: $\mathrm{p} 53$ appears to play a major role in adenoma-like, terminally differentiated, and mucinous precursors in IBD related neoplasia, whereas combined alterations of p53 and $\beta$-catenin are associated with serrated precursors. As aberrant $\mathrm{p} 53$ is most commonly seen in HGD, it likely represents a late event in IBD carcinogenesis. While MUC2 is expressed by most dysplasia subtypes, MUC6 is a marker of hypermucinous phenotype.

\section{Immune Microenvironment and Clinical Outcomes in Gastric} Carcinoma

Pallavi A Patil, Li J Wang, Kara A Lombardo, Thomas J Miner, Andres Matoso. Brown University and Rhode Island Hospital, Providence, RI; Brown University and The Miriam Hospital, Providence, RI.

Background: Tumor microenvironment is gaining importance as a driver of tumor behavior in several tumor types. We studied expression of immunomodulating proteins in gastric carcinomas including their relationship with microsatellite instability (MSI). Design: Tissue microarray were constructed with gastrectomies from 6/2003 to 12/2013. Immunohistochemistry for PD-L1, IDO, WARS, CD3 tumor infiltrating lymphocytes (TIL), and MSI markers were performed. Staining was scored by intensity (mild $=1$, moderate $=2$, strong $=3$ ) and extent $(5-25 \%=1,25-50 \%=2,>50 \%=3)$; combined score of $\geq 4$ was considered positive. TIL were counted per 100 epithelial cells, and per HPF for stroma. Clinicopathological parameters were recorded.

Results: The study included 86 patients. Average age was 71 (41-90), tumor size 5.8 $\mathrm{cm}(0.5-18.5)$. Median follow-up was 34 months (0-148), and median time to disease prgression was 13 months (2-52). Tumor types were $45 \%$ tubular, $38 \%$ diffuse, $17 \%$ mixed. Metastasis and recurrence $(41 \%)$ were associated with lymphovascular invasion (58\%) (Pearson $\mathrm{r}=0.24, \mathrm{p}=0.026$ ). PD-L1(-) tumors presented at higher stage ( $\mathrm{pT} 4 \mathrm{~b})$ $(19 \%$ vs. $3 \% ; \mathrm{p}=0.02)$ and showed more perineural invasion $(73 \%$ vs. $40 \%, \mathrm{p}=0.009)$. $\mathrm{IDO}(+)$ tumors were associated with less recurrence $(30.0 \%$ vs. $57.1 \%, \mathrm{p}=0.012)$ and less metastasis ( $22 \%$ vs $46 \%, \mathrm{p}=0.032$ ). WARS strongly positive tumors had less PNI $(40 \%$ vs $69 \%, p=0.013)$. There was no association between WARS and IDO expression (Pearson $\mathrm{r}=0.085, \mathrm{p}=0.46$ ). MSI unstable tumors were associated with higher numbers of stage T4b (22\% vs $4 \%, \mathrm{p}=0.03)$. Average CD3(+) TILs were $9 / 100$ epithelial cells and in stroma 159/HPF. There was correlation between PD-L1 expression by tumor epithelium and TILs (Pearson $\mathrm{r}=0.35, \mathrm{p}=0.001$ ). Neither PD-L1, WARS nor MSI differed with sex, histologic subtype or progression.

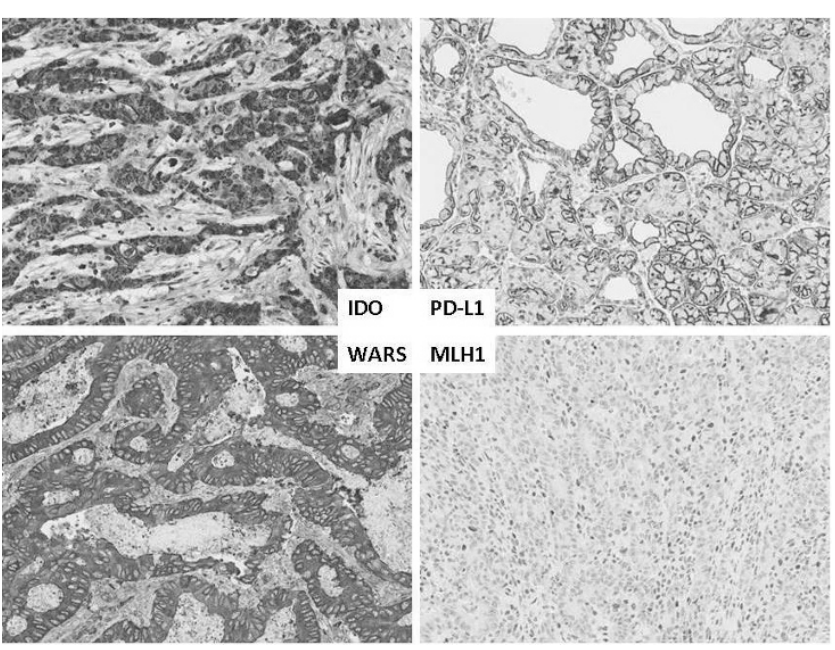

Conclusions: Our results suggest that tumor microenvironment plays a role in gastric cancer behavior. PD-L1 negative and MSI unstable carcinomas were associated with histopathologic features of poor outcome, while IDO positive tumors were associated with less progression.

778 Assessability of Endoscopic Biopsies of the Proximal Large Intestine for the Changes Diagnostic of Sessile Serrated Polyp/Adenoma Garrison Pease, Curtis R Hall. University of Chicago - NorthShore, Evanston, IL. Background: Given that the diagnostic criteria for sessile serrated polyp/adenoma (SSP/A) imply an ability to observe crypt bases in endoscopic biopsies (EB) of the large intestinal mucosa (LIM) (in search of the characteristic features of SSP/A), we sought to develop an understanding of the assessability for SSP/A of such biopsies in our surgical pathology files.

Design: Through search of our files and review of slides generated from biopsies of the proximal LIM, we identified cases, from a single month, in the serrated polyp spectrum (hyperplastic polyp (HP) through SSP/A) for inclusion in this study. One or both of us queried each eligible case for number of lesional crypt bases present and number of crypts with features diagnostic for SSP/A in relevant biopsies.

Results: Our results are presented in the bar graphs below:
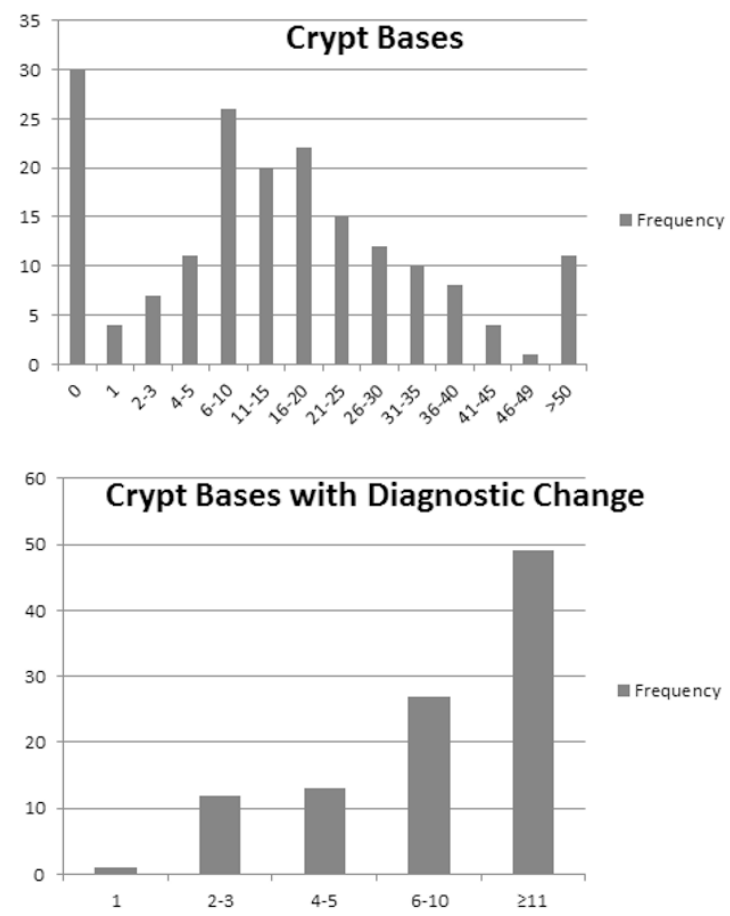

In summary, of 182 serrated lesions identified, 30 (16.5\%) had no crypt bases available for review, while $52(28.6 \%)$ had 5 or less. Of 102 cases judged diagnostic of SSP/A, $13(12.7 \%)$ had only 1 to 3 crypt bases with changes diagnostic for SSP/A.

Conclusions: These results indicate that the diagnosis of SSP/A is subject to sampling error in regards to the inclusion of crypt bases, wherein are most of the features characteristic of SSP/A. Sampling error, along with interobserver interpretive variation (a source of error not addressed in this study), is likely to present, in day-to-day signout, a persistent barrier to completely accurate assessment for the presence or absence of SSP/A in any one patient through histopathological review of LIM biopsies. We conclude, as others have through other methodologies, that the identification of patients 
at risk for development of LIM adenocarcinoma through the serrated polyp pathway should depend as much on endoscopic assessment of the number and size of serrated polyps as much as on the histopathological assessment of these lesions.

\section{ERBB2 Alterations a New Prognostic Biomarker in Stage II Colon Cancer (CC) from a FOLFOX Based Adjuvant Trial (PETACC8)}

Frederique Penault-Llorca, Pierre Laurent-Puig, Anne Cayre, Karine Le Malicot, JeanFrancois Emile, Julien Taieb. Centre Jean Perrin, Clermont Ferrand, France; Hôpital Européen Georges Pompidou, Paris, France; Fédération Francophone de Cancérologie Digestive (FFCD, Dijon, France; Hopital Ambroise Paré, Boulogne, France; Hopital European George Pompidou, Paris, France.

Background: $E R B B 2$ amplifications have been recently shown as a potential targetable alteration in metastatic colorectal cancer, in the HERACLES trial. This discovery reinforces the interest to study the occurrence and the prognostic role of ERBB2 alterations in stage III colon cancer (CC) where we need to improve adjuvant strategies. Prospective collection of PETACC8 study [EudraCT number 2005-003463-23] allowed us to evaluate the occurrence and the prognostic impact of $E R B B 2$ alteration.

Design: From the 2559 pts of PETACC8 trial, 2043 signed the translational research informed consent. Among them, tissues samples were available in 1795 pts for NGS screening, and 1804 were available for immunochemistry and FISH analysis. We searched for ERBB2 mutation in exon 19 to 21 and for amplification, using the colon lung cancer panelbV2 and an algorithm previously validated. All cases were screened by immunohistochemistry (IHC) with PATHWAY anti-HER-2/neu (4B5) from Ventana and by FISH (zytolight SPEC ERBB2/CEN17 dual color) on TMAs.

Results: Altogether, ERBB2 alterations were present in 64 pts $(3.8 \%)$ and in $42(5,6 \%)$ in the $K R A S$ wild type group. We identified 17 mutations (1\%), most frequent p.V842I (5 pts), p.V777L (3 pts), p.L755S (3 pts). No significant association with $R A S$ or $B R A F$ mutations nor mutual exclusivity. We identified NGS ERBB2 amplification in 49 pts $(2.9 \%), 46$ by FISH (including the 39 IHC $3+$ ). Discordances were observed in the $0 / 1+$ category, 4 being amplified by FISH and NGS. Prognostic impact of $E R B B 2$ alterations by pooling pts mutated or amplified: in univariate analysis, ERBB2 alterations were associated with shorter time to recurrence (HR: 1.55 [95\%CI: $1.02 ; 2.36] \mathrm{p}=0.04$ ) and shorter overall survival (HR: $1.57[0.99 ; 2.5] \mathrm{p}=0.05$ ). This prognostic value was maintained after adjustment for treatment, $R A S$ mutation, histological grade, tumor location, $\mathrm{pT}$ and $\mathrm{pN}$ status, bowel obstruction or perforation and embolies.

Conclusions: $E R B B 2$ alteration is a rare event found in approximately $4 \%$ of stage III CC pts. Its poor prognostic value supports the evaluation of anti-ERBB2 therapies in the adjuvant setting. Due to their low frequency, screening for those alterations by NGS should show to be more cost effective than IHC and FISH.

\section{$780 \quad$ Epstein-Barr Virus Infection in Refractory Inflammatory Bowel Disease Patients Undergoing Colectomy}

Maryam Pezhouh, Ogechukwu Pearl Eze, Yaman Tarabishy, Kevin Waters, Alyssa Parian, Mark Lazarev, Elizabeth A Montgomery, Lysandra Voltaggio. Johns Hopkins Hospital, Baltimore, MD.

Background: Inflammatory bowel disease (IBD) is a chronic inflammatory process of unknown etiology. A potential role for viral infection and associated mucosal immune response has been suggested by some studies. Infection with Epstein-Barr Virus (EBV) can elicit a brisk mononuclear response in the gastrointestinal tract. The role of this infection in patients with IBD refractory to treatment has not been fully evaluated.

Design: Surgically resected colonic specimens from 52 patients with refractory IBD (48 with ulcerative colitis, 3 patients with Crohn's disease and 1 patient with indeterminate colitis) were retrieved from our archive. Eleven colectomy specimens from ulcerative colitis patients who had undergone resections due to the presence of dysplasia or endometriosis were included as controls. Highly sensitive EBV-encoded small RNA1 (EBER-1) in situ hybridization was performed on a representative block from each specimen. EBER-1 reactivity was graded as absent, focal, or diffuse.

Results: EBV was detected in $63.5 \%$ (33 out of 52 ) of patients with refractory IBD, compared to $18 \%$ ( 2 out of 11 ) of the control group (P-value $<0.001$ ). Focal EBER-1 positivity was present in $50 \%$ of cases of refractory IBD ( 26 out of 52 ) compared to $18.2 \%$ of controls ( 2 out of 11 ). Diffuse EBER-1 reactivity was seen in samples from 7 of 52 patients $(13.5 \%)$ in the refractory IBD group. None of the samples from the control group patients had diffuse EBER-1 positivity. Patients with EBER-1 positivity were older (average age of 42 years old) compared to patients without EBER-1 reactivity(average age, 32 years old) $(\mathrm{P}-\mathrm{value}=0.039)$. There was no gender difference. Conclusions: Our findings suggest a potential role for EBV infection in patients who are refractory to IBD treatment. More studies are needed to confirm our observations.

781 Lymphocytic Gastritis: Clinicopathologic Associations, Old and New

Maryam Pezhouh, James A Miller, Danielle Hutchings, Maryam Shabihkhani, Ogechukwu Pearl Eze, Lysandra Voltaggio, Elizabeth A Montgomery. Johns Hopkins Hospital, Baltimore, MD.

Background: Lymphocytic Gastritis (LG) is a pattern of gastric injury characterized by gastric intraepithelial lymphocytosis. Reported associations include celiac disease, Helicobacter pylori (HP) infection, and other conditions. We aimed to explore the clinical associations of LG in our patient population.

Design: We searched our pathology database from 1984 to 2016 and found 333 patients diagnosed with LG. Clinical, demographic, and histological findings were reviewed. Results: Cases were derived from 193 females (58\%) and $140(42 \%)$ males with a mean age of 48 years. Associated conditions were identified in 211 patients $(63.4 \%)$ and included celiac disease $(\mathrm{N}=57,27 \%)$, HP infection ( $\mathrm{N}=51,24.6 \%)$, and Crohn's disease $(\mathrm{N}=12,5.7 \%)$. Eleven patients $(5.2 \%)$ had adenocarcinoma involving the stomach, esophagus or pancreas. Three patients had gastric lymphoma (1.4\%). Sixty eight (32.2\%) patients were taking multiple medications that have been associated with GI tract injury including high dose NSAIDs ( $\mathrm{N}=22,32 \%)$, angiotensin receptor II antagonists such as olmesartan, irbesartan, and losartan $(\mathrm{N}=20,24 \%)$, and selective serotonin reuptake inhibitors $(\mathrm{N}=9,13.2 \%)$. Other medications included levothyroxine, statins, and proton pump inhibitors. Interestingly, patients with celiac disease were significantly younger (mean age of 27 years) than those with $H$. pylori and medication associated LG (mean age of 51 and 58 years, respectively) (P-values $<0.05$ ).

Conclusions: The clinical associations in our patient population mirror those reported by others but, in addition, underscore the potential role of several classes of medications in the development of this pattern of injury.

\section{Spectrum of Colonic Findings in Renal Transplant Patients} Meredith Pittman, Jose Jessurun, Rhonda K Yantiss. Weill Cornell Medicine, New York, NY.

Background: Solid-organ transplant recipients often have gastrointestinal (GI) symptoms necessitating colonoscopy. Biopsies may be normal or show a range of inflammatory changes, including features that mimic idiopathic inflammatory bowel disease (IBD). The purpose of this study was to assess colonic biopsies from renal transplant patients and to correlate histologic features with clinical findings.

Design: The pathology database was queried and 91 renal transplant patients were identified who had colonic biopsies during a 15-year period (2001-2016). Of these, 40 patients were excluded from analysis: 16 were asymptomatic and undergoing screening colonoscopy, and 24 had no clinical follow-up. The remaining $51(56 \%)$ patients had GI symptoms or significant histologic findings and comprised the study group. Histology from these patients was correlated with clinical history, medication usage, and colonoscopic findings.

Results: There were 27 men and 24 women in the study group. Most patients presented with diarrhea (58\%), followed by anemia/bleeding (23\%). Active colitis was present in $40 \%$ of cases, $27 \%$ showed crypt architectural distortion, and $15 \%$ showed prominent crypt epithelial cell apoptosis. Normal findings were present in $23 \%$ of cases

Fifteen $(29 \%)$ patients had an acute self-limited colitis: 10 proved to have an infectious etiology, 2 had non-steroidal anti-inflammatory drug injury, and 3 had injury attributed to either chemotherapy, mycophenolate, or lactose intolerance (one case each).

Crypt architectural distortion was seen in $14(27 \%)$ cases. Six of these were classified as chronic active colitis, including the all 3 patients who proved to have IBD, 2 with mycophenolate injury, and one with small intestinal bacterial overgrowth (SIBO). Five patients with crypt distortion and crypt cell apoptosis proved to have mycophenolate injury $(\mathrm{n}=3)$, tacrolimus injury $(\mathrm{n}=1)$, or $\operatorname{SIBO}(\mathrm{n}=1)$. Crypt architectural distortion was an isolated finding in 3 patients: one had mycophenolate injury, one had diverticulosis, and one had SIBO.

Of the $12(23 \%)$ patients with normal colonic mucosa, 6 proved to have drug-induced GI symptoms, including 3 receiving mycophenolate.

Conclusions: Mucosal inflammation is common in colonic biopsies from renal transplant patients with GI symptoms, and nearly 1/3 of biopsies show crypt architectural distortion or chronic colitis. Most of these patients do not have and do not develop IBD. Those with active colitis alone are more likely to have an infectious etiology, whereas chronic changes are more often attributable to a drug injury, particularly mycophenolate.

783 Ampullary Region

Sonya Purushothaman, Helen E Remotti, Stephen M Lagana, Susan J Hsiao, Hui-Min Yang. Columbia University, New York, NY.

Background: GATA3 binding protein is a transcription factor essential in the differentiation and proliferation of breast and urothelial epithelium, certain T-cell populations, hair follicles, trophoblast, and vascular endothelium. Its expression is routinely utilized in surgical pathology to identify carcinomas of breast and urothelial origin. Ampullary carcinomas encompass a distinct albeit histologically heterogenous group of neoplasms. Metastasis to the ampulla originating from either the breast or the urothelium, while rare, has been reported in literature. The immunohistochemical staining pattern of GATA3 has not been previously established in ampullary adenocarcinoma. We therefore sought to determine the frequency of GATA3 positivity in ampullary adenocarcinoma.

Design: Staining for GATA3 was performed on 38 adenocarcinomas from the ampullary region, including well, moderately, and poorly differentiated tumors with intestinal, pancreatobiliary, and unclassifiable morphology. Positive staining was defined as a minimum of $5 \%$ of tumor cells with nuclear staining. The staining intensity was further characterized as weak $(1+)$, moderate $(2+)$, or strong $(3+)$. Intralesional lymphocytes served as an internal $3+$ control. Non-neoplastic small intestinal mucosa, pancreatic adenocarcinoma, and extrahepatic bile duct adenocarcinomas were also used as control groups.

Results: GATA3 stained 4 of $38(11 \%)$ ampullary adenocarcinomas. None of the positive cases exhibited strong diffuse staining. The positive staining was limited to nuclear staining in a median of $5-10 \%$ of tumor cells and up to $50 \%$ of tumor cells, with a staining intensity of $1+$ to $2+$. Of note, positive staining was only seen in tumors that were moderately to poorly differentiated. Non-neoplastic small intestinal mucosa was negative for GATA3 in the 38 cases examined $(0 \%)$. Pancreatic adenocarcinoma exhibited positive staining in 2 of 18 cases $(11 \%)$. Extrahepatic bile duct adenocarcinoma showed no positive staining in the 20 cases examined $(0 \%)$.

Conclusions: Given the low frequency and limited positivity of GATA3 immunohistochemical staining in ampullary adenocarcinoma, GATA3 may be helpful in distinguishing metastatic malignancy to the ampulla from primary ampullary adenocarcinoma, especially in cases showing atypical histology and lacking an identifiable in situ component. 
784 Neuroendocrine Neoplasms of the Gallbladder. An Immunohistochemical and Clinicopathologic Analysis of 29 Cases

Michelle D Reid, Juan Carlos Roa, Bahar Memis, Takashi Muraki, Juan Carlos Araya, Miguel Villaseca, Hector Losada, Juan M Sarmiento, Gabriel Sica, Volkan Adsay, Emory Uni, Atlanta, GA; Pontificia Uni Católica de Chile, Santiago, Chile; Uni de La Frontera, Temuco, Chile.

Background: Neuroendocrine neoplasms of GB are poorly characterized. While new insights have been gained into the pathogenesis, classification and prognostication of GI NENs it is not known whether these are also valid for GB.

Design: 29 GB NENs were identified in a systematic review of of 606 consecutive GB carcinomas (GBCs) in authors' files. Blocks were available for IHC in 18 .

Results: Mean age, 59(26-93). F/M:22/5=4.6. Only 1 was morphologically "well differentiated" (WDNET); 28 were poorly differentiated neuroendocrine carcinoma (PDNEC/GBNEC). Median Ki67 for PDNECs was $75 \%$ (40-95) with 1 PDNEC $<50 \%$. Five $(17 \%)$ had intracholecystic papillary tubular neoplasm component. 12 PDNECS were pure ( 7 small cell, and 5 large cell). 16 cases were mixed with adenocarcinoma and the NE component was predominant in all but one. Tumors were positive for NE markers (synaptophysin $94 \%$, chromogranin $71 \%$, CD56 94\%), broad spectrum keratins (AE1/3 94\%, Cam5.2 88\%), often with upper GI profile (CK7 67\%; CK20 0\%). TTF1 positivity was in $31 \%$ (all small cell). Molecular abnormalities recently shown to be specific for PDNECs (vs WDNETs), namely Rb loss and $\mathrm{p} 53$ over-expression, were detected in $56 \%$ and $24 \%$, respectively. Meanwhile, pathways linked to WDNETs (but preserved in PDNECs), namely ATRX and DAXX, were retained in all but $6 \%$ which showed ATRX loss. SMAD4 loss (characteristic of distal pancreatobiliary adenocarcinomas), was detected in $13 \%$ (with proper inbuilt controls). Overall, median survival was 9 mo, and 5 -yr, $19 \%$ vs $44 \%$ of GBC ( $\mathrm{p}=0.047$ ). In this limited data, $\mathrm{Rb}$ loss was associated with better outcome ( $\mathrm{p}=0.046$ ), and strong $\mathrm{NE}$ expression (synaptophysin $[\mathrm{p}=0.035]$ $\mathrm{CD} 56[\mathrm{p}=0.04])$ were associated with better outcome

Conclusions: NE differentiation is uncommon in $\mathrm{GBC}(<4 \%)$. Most are PDNECs that are high grade morphologically, proliferatively (median Ki67, $75 \%$, all but one $>50 \%$ ), and molecularly ( $>50 \%$ with Rb loss and/or p 53 over-expression), and prognostically (median surv, 9 mos). Accurate diagnosis can be confirmed with NE markers along with CKs, the latter showing more upper-GI profile. Similar to other GI NECs, they seem to be neuroendocrine differentiated version of glandular neoplasms (not uncommonly admixed with $\mathrm{HGD} / \mathrm{CIS}$ or invasive adenoca), rather than higher grade transformation of WDNETs; alteration of ATRX/DAXX, a common finding in WDNETs, is not a feature. As such GBNECs should be considered candidates for cis-platinum protocols employed for PDNECs in the GI tract.

\section{$785 \quad$ Molecular Profiling of Dysplasia Subtypes in Inflammatory} Bowel Disease: A Comparative Analysis with Sporadic Precursors of Colorectal Carcinoma

Elizabeth M Rinehart, Jonathan A Nowak, Neal I Lindeman, John R Goldblum, Robert D Odze, Deepa T Patil. Brigham and Women's Hospital, Boston, MA; Cleveland Clinic, Cleveland, $\mathrm{OH}$.

Background: Recent studies indicate that inflammatory bowel disease (IBD)-associated colorectal cancers (CRC) show different biologic characteristics and molecular alterations compared to sporadic CRC. There is little information on the molecular alterations in IBD precursors. The aim of this study was to perform sequencing analysis on different morphologic sutypes of IBD-related dysplasia in order to explore potential pathways of carcinogenesis and to compare IBD-related dysplasia to sporadic precursors from non-IBD patients.

Design: 29 low-grade dysplastic (LGD) lesions from 24 patients (8 M, 16 F) formed the study cohort. There were 6 tubular adenomas, 5 sessile serrated polyps with LGD and 18 IBD-related LGD (13 adenoma-like, 3 serrated, 2 hypermucinous). DNA from macrodissected FFPE tissue was analyzed using the OncoPanel platform, a hybrid-capture-based NGS assay that interrogates 300 cancer genes for mutations and copy number variations. Sequencing was performed using an Illumina HiSeq 2500 sequencer. Data was analyzed via a customized pipeline that incorporates open source and internally developed tools.

Results: Alterations in the key colorectal carcinogenesis genes $A P C$ and $K R A S$ were common in both sporadic and IBD-related dysplasia. Activating KRAS alterations occurred in $27 \%$ of sporadic and $44 \%$ of IBD-related dysplasia; loss of function $A P C$ alterations occurred in $45 \%$ of the former and $50 \%$ of the latter. There were no significant differences in mutation frequency between the two groups $(\mathrm{OR}=0.5, \mathrm{p}=0.45$ for $K R A S$; $\mathrm{OR}=0.8, \mathrm{p}=1.0$ for $A P C$ ). $T P 53$ alterations were rare (only present in one case of IBDrelated dysplasia). CTNNBI ( $\beta$-catenin) alterations were more frequent in sporadic compared to IBD-related dysplasia $(\mathrm{OR}=12.7, \mathrm{p}=0.018)$; however, this association was not significant when adjusted for multiple hypothesis testing. Additional analysis showed that ARIDIA alterations were common in adenoma-like IBD dysplasia (31\%), but not in sporadic adenomas or other subtypes of IBD-related dysplasia.

Conclusions: There is significant overlap in the molecular phenotype of sporadic and IBD-related dysplasia, with alterations in KRAS and $A P C$ commonly found in both groups. TP53 alterations are uncommon across a range of LGD, including those arising in IBD, suggesting that TP53 alteration is a late event in neoplastic progression in both groups. ARIDIA alterations are a feature of adenoma-like IBD dysplasia, but not of sporadic adenomas.
786 Expression of PD-1 and PD-L1 in High Grade Neuroendocrine Carcinomas of the Gastrointestinal Tract and Associated Host Immune Cells: A Potential Target for Anti-PD-1/PD-L1 Therapy

Jordan A Roberts, Raul S Gonzalez, Chanjuan Shi. Vanderbilt University Medical Center, Nashville, TN; University of Rochester Medical Center, Rochester, NY. Background: High grade Neuroendocrine Carcinomas (HGNECs) are aggressive tumors that often have limited treatment options. The programmed death 1 (PD-1) pathway plays critical role in the host immune response to cancer. Programmed death 1 ligand (PD-L1) and receptor (PD-1), expressed on tumor or immune cells, serve as a marker for potential clinical response to immune checkpoint blockade using antiPD-1/PD-L1 therapies. Therefore, it may be advantageous to investigate the complex tumor-host immune milieu, through the expression of PD-1 and PD-L1 in immune and tumor cells, in HGNECs.

Design: Forty-two HGNECs at various sites of the digestive system (colon, small bowel, pancreas, stomach, esophagus, gallbladder) were identified from 42 patients in our departmental archives between 1/1/97 and 6/30/15. Patient medical records, pathology reports, and pathology slides were reviewed. Immunohistochemistry for PD-1 and PD-L1 was performed on all cases (3 biopsies and 39 resections). PD-1 and PD-L1 positivity was defined as $\geq 1 \%$ cell surface expression of PD-1 and PD-L1 by tumor cells and/or intra- and peri-tumoral immune cells (lymphocytes and histiocytes). Results: Mean age of the patients was 61.8 years, ranging from 32 to 80 . Overall 16 of $42(38.1 \%)$ cases had PD-L1 expression by tumor cells and/or inflammatory cells. Nine of 42 cases $(21.4 \%)$ were positive for PD-L1 expression in tumor cells and twelve of $42(28.6 \%)$ were positive PD-L1 expression in histiocytes. $33.3 \%$ of cases $(14 / 42)$ showed PD-1 expression in macrophages and 45.2\% (19/42) showed PD-1 positive lymphocytes. Five of 42 cases $(12.0 \%)$ showed co-positivity of PD-L1 in tumor cells and PD-1 in immune cells. Seventeen of the cases (40.5\%) did not stain for PD-L1 or PD-1 in the tumor cells or in the intra-/peri-tumoral immune cells. Mean disease specific survival for PD-L1 positive and negative tumors was 24.7 and 25.4 months, respectively. Conclusions: The aggressive nature of HGNECs and limited treatment options necessitates identifying novel treatments. The promises shown in anti-PD-1/PD-L1 therapies in lung cancer and melanoma opens the possibility for treatment of other tumors. Our data suggest that tumor-immune system interface may play an important role in HGNECs and anti-PD-1/PD-L1 therapies could be considered a therapeutic option in select patients with HGNECs.

\section{Adenocarcinoma Ex-Goblet Cell Carcinoid of the Appendix Is} a Tumor of Intestinal Lineage and Mucinous Differentiation

Brian Robinson, Bahar Memis, Andy Toussaint, Yue Xue, Alyssa Krasinskas, Charles Staley, Joshua H Winer, Maria C Russell, Walid Shaib, Bassel El-Rayes, Volkan Adsay, Michelle D Reid. Emory, Atlanta, GA.

Background: Appendiceal adenocarcinoma ex-GCC (XGC) is a highly aggressive tumor of debated nature and origin, that has been shown to have very high propensity for intraabdominal carcinomatosis and "Krukenberg" type spread to gynecologic tract (PMID: 27338636), and thus commonly falls in the differential diagnosis with gastric and other signet ring carcinomas. The data regarding the immunophenotypic differentiation and cell lineage of XGC is highly limited with conflicting results.

Design: Upon IRB approval, immunohistochemistry (IHC) for cell lineage markers, including CK7, CK20, MUC1, MUC2, MUC5AC, MUC6 and CEA was performed in 49 XGCs. The extent of positivity was scored as either focal $(<25 \%)$, moderate $(25-75 \%)$, strong $(75-90 \%)$, or diffuse (90-100\%). Additionally, IHC stains for E-cadherin and beta-catenin were performed, and the extent and subcellular localization were noted. Results: Intestinal differentiation marker CDX2 was commonly expressed ( $98 \%$ at least moderate) and so was the "goblet cell marker", MUC2 (76\% at least moderate) along with mucin oncoprotein CEA ( $97 \%)$; however, unlike sporadic colonic adenocarcinomas, beta-catenin nuclear expression was virtually non-existent (positive in 1/44 cases examined). The cytokeratin profile was also that of a lower GI tract neoplasm, with frequent CK20 positivity ( $90 \%$ at least moderate) and frequent absence of CK 7 (detected in only $16 \%$ ). Unlike gastric signet-ring cell (poorly cohesive) carcinomas, membranous E-cadherin expression was maintained in a majority of cases ( $90 \%$ strong membranous). Markers of aggressive behavior in GI/pancreatobiliary cancers, MUC1 and MUC5AC, were expressed in only 11 and 20\%, respectively. MUC6 (pyloric gland marker) was mostly negative (only 1 case was positive).

Conclusions: XGC is characterized by intestinal lineage and mucin production. IHC markers can help to distinguish this tumor type from its main differential in the abdomen, gastric signet ring cell carcinoma. Despite its high grade and rapidly disseminative nature, markers of aggressive behavior in GI tract, MUC1 and MUC5AC, are infrequently expressed. This study also further supports the fact that this entity is an adenocarcinoma, showing all the features of an adenocarcinoma at the IHC level as well, including diffuse/strong CEA positivity, and therefore, lending further support for the proposed term appendiceal "crypt cell adenocarcinoma".

\section{Invasive Adenocarcinoma Arising from Low-Grade Appendiceal} Mucinous Neoplasm: Clinicopathologic Features

Nemencio Ronquillo, Mark A Valasek. University of California San Diego, San Diego, CA.

Background: Primary appendiceal mucinous lesions are uncommon and represent a spectrum from non-neoplastic mucous retention cysts to invasive adenocarcinoma. The precise nomenclature and management of various appendiceal mucinous lesions is controversial. Here, we describe cases of invasive appendiceal adenocarcinoma arising in a background of low grade appendiceal mucinous neoplasm (LAMN).

Design: The pathology database was searched to identify cases of adenocarcinoma arising in the appendix with background LAMN in the last 2 years based on criteria summarized in the 2016 Peritoneal Surface Oncology Group International Consensus. Clinicopathologic features were then assessed. 
Results: Eight patients $(6 \mathrm{M}, 2 \mathrm{~F})$ with a mean age of 53 years (range $33-70$ years) were identified. Three patients underwent hemicolectomy (37.5\%), 4 (50\%) underwent appendectomy and completion hemicolectomy, and 1 patient underwent appendectomy alone $(12.5 \%)$. In the 4 patients who underwent completion hemicolectomy after appendectomy, $3(75 \%)$ had residual disease in the appendiceal stump/right colon despite having negative surgical margins on the appendectomy specimen. The average size of the background LAMN was $6.3 \mathrm{~cm}(3.4-13 \mathrm{~cm}, \mathrm{n}=8)$ and the invasive component was $4 \mathrm{~cm}(1-7.5 \mathrm{~cm}, \mathrm{n}=4)$. Two cases $(25 \%)$ showed transition of LAMN to high grade appendiceal mucinous neoplasm (HAMN). Peritoneal dissemination was identified in 7 patients $(87.5 \%)$ and 5 of them $(62.5 \%)$ had diffuse peritoneal disease. Seven $(87.5 \%)$ had pT4 and $1(12.5 \%)$ had pT3 disease. Of the 3 patients $(37.5 \%)$ with nodal disease, $2(25 \%)$ had $\mathrm{pN} 1$ and $1(12.5 \%)$ had $\mathrm{pN} 2$ disease. Of the 3 patients in whom ancillary tests were performed, all had KRAS mutations and were microsatellite stable, and $1(33 \%)$ had a GNAS mutation. Six $(75 \%)$ were staged as IVB, $1(12.5 \%)$ as IVA, and $1(12.5 \%)$ as IIB. All patients with IVB disease underwent cytoreduction, heated intraperitoneal chemotherapy, and systemic chemotherapy. All patients were alive at last follow up (mean 12 mos; range 2-25 mos): 5 (62.5\%) with no evidence of disease and $3(37.5 \%)$ with disease.

Conclusions: Invasive adenocarcinoma of the appendix can be seen as a distinct lesion arising from LAMN. Patients with invasive adenocarcinoma arising from LAMN are more likely to be male and tend to present with advanced disease. Additional studies are needed to better understand the progression of LAMN and to incorporate the natural history of these neoplasms into more refined staging systems.

789 Immune Checkpoint Expression and the Tumor Immune Environment in Colorectal Carcinoma

Matthew W Rosenbaum, Jacob R Bledsoe, Marina Kem, Tiffany Huynh, Mari MinoKenudson. Massachusetts General Hospital, Boston, MA; University of Massachusetts Medical School, Worcester, MA.

Background: Programmed Cell Death Protein 1 axis blockade enhances anti-tumor immunity, leading to clinical response in microsatellite instability (MSI)-high colorectal cancer (CRC). Tumor cell expression of Programmed Cell Death Ligand 1 (PD-L1) is associated with treatment response in various tumor types. The association and biological role of additional, potentially actionable immune inhibitory targets, such as Indoleamine 2,3-Dioxygenase 1 (IDO1) and B7-H4, is not well understood in CRC. Design: A tissue microarray consisting of multiple cores from 171 cases of CRC was analyzed for PD-L1, IDO, and B7H4 expression on tumor cells by immunohistochemistry using $\mathrm{H}$-scores (graded as percentage of cells expressing $0,1+$, $2+$, or 3+ staining for a total of 0-300). CD8, TBET (a Th1 pathway activation marker), and GATA3 (a Th2 pathway activation marker)-positive tumor infiltrating lymphocytes (TILs) were dichotomized (abundant or not). For each case, clinicopathologic data and follow-up was reviewed.

Results: Using a cut-off $\mathrm{H}$ score of $\geq 5,18(11 \%)$ cases expressed both PD-L1 and IDO1, 14 (8\%) expressed IDO1 only, 11 (6\%) expressed PD-L1 only, and $128(75 \%)$ did not express either. No cases showed positive B7H4 expression. Compared to PD-L1/ IDO negative tumors, PD-L1-only expressing tumors were associated with abundant TBET+ TILs $(\mathrm{p}<0.02)$, TP53 mutation $(\mathrm{p}<0.03)$, and medullary phenotype $(\mathrm{p}<0.03)$. Compared to PD-L1/IDO negative tumors, IDO-only expressing tumors occurred in older patients $(\mathrm{p}<0.03)$, primary resections lacking pre-collection therapy $(\mathrm{p}<0.02$ each), and had abundant CD $8+$ and TBET + TILs ( $<<0.005$ for both), with a medullary phenotype $(\mathrm{p}=0.01)$ and MSI $(\mathrm{p}<0.001)$. Clinopathologic features of IDO1 and/or PDL1positive tumors overlapped those of IDO1-only expressing tumors. On univariate survival analysis, PD-L1, IDO and B7H4 had no bearing on survival.

Conclusions: IDO1 expression with or without PD-L1 expression is common in CRC, and is associated with the immune microenvironment and MSI that have been reported in association with PD-L1 expression. Thus, IDO1 may be a mechanism of acquired immune resistance providing normally immunogenic colorectal carcinoma a means of immune evasion. Thus, blockade of IDO1 may represent a potent therapeutic strategy to reactivate anti-tumor immunity in CRC. In addition, the difference in TP53 mutation rate between IDO+ and PD-L1+ populations implies that a different pathogenic mechanism may underlie the expression of these molecules.

\section{HER2 Driven Colorectal Cancer: A Comprehensive Genomic} Profiling Study

Jeffrey Ross, Siraj M Ali, Julia A Elvin, Alexa Schrock, James Suh, Jo-Anne Vergilio, Shakti H Ramkissoon, David Fabrizio, Garrett Frampton, Vincent Miller, Philip Stephens, Laurie M Gay. Albany Med Col, Albany, NY; Foundation Med, Cambridge, NY.

Background: In contrast with lung cancer, the development of precision treatment for colorectal cancer (CRC) has only recently begun to achieve recognition. One CRC target that is rapidly emerging through active clinical trials is ERBB2 (HER2).

Design: DNA was extracted from 40 microns of FFPE from 8,199 consecutive relapsed refractory and metastatic CRC. Comprehensive genomic profiling (CGP) was performed using a hybrid-capture, adaptor ligation based next generation sequencing assay to a mean coverage depth of $>600 \mathrm{X}$. Tumor mutational burden (TMB) was calculated from a minimum of $1.2 \mathrm{Mb}$ of sequenced DNA as previously described and reported as mutations/ $\mathrm{Mb}$ of sequenced DNA. The results were analyzed for all classes of genomic alterations (GA), including base substitutions, insertions and deletions (short variants), fusions, and copy number changes including amplifications (amp) and homozygous deletions.

Results: Of 8,199 CRC profiled, 265 (3.2\%) featured ERBB2 amp, 165 (2.0\%) ERBB2 SV and $4(0.5 \%)$ harbored $E R B B 2$ fusions with $400(4.9 \%)$ total CRC having at least 1 ERBB2 GA. Median ERBB2 copy number was 26 (range 6 to 242) in the amplified patients. In $165 \mathrm{CRC}$ a total of 177 ERBB2 SV were identified, 172 (97\%) of which were considered to be activating. One hundred eitghty (45\%) of the patients were female and $220(55 \%)$ were male. The mean age was 58.4 years (range 25 to 91 years). The genes most frequently co-altered with $E R B B 2$ were SV GA in $T P 53(82 \%), A P C(70 \%)$, KRAS (26\%), SMAD4 (15\%) and PIK3CA (13\%). TOP $2 A$ was the most frequently co-amplified gene $(21 \%)$. Clinically relevant GA significantly under-represented in $E R B B 2$-altered CRC included $K R A S$ at $26 \%(\mathrm{p}=0.001)$ and $B R A F$ at $4 \%(\mathrm{p}=0.003)$ as well as other kinases at $1 \%$ including EGFR, KIT, MET and RET. TMB (9.9 vs 8.0 mean mut $/ \mathrm{Mb}$ ) and the frequency of MSI-high status (2.9\% vs $2.4 \%)$ in $E R B B 2$-driven CRC were higher in ERBB2 altered CRC compared with ERBB2 WT CRC. Multiple examples of major patient responses to anti-HER2 targeted therapies including both $\mathrm{CRC}$ with ERBB2 amplification and SV will be presented.

Conclusions: Although lower than observed in breast and upper gastrointestinal carcinomas where anti-HER2 therapies are approved indications, the frequency of $E R B B 2 \mathrm{GA}$ in $\mathrm{CRC}$ at $4.9 \%$ is significant. Importantly, nearly half of CRC ERBB2 alterations are not detectable by routine IHC and FISH testing. However, the success of anti-HER2 therapies shown here and progress in on-going clinical trials indicates that targeting $E R B B 2$ will likely become an approved advance in precision therapy for CRC patients.

\section{Is Serrated Polyposis a Genetic Condition?}

Christophe Rosty, Mark Clendenning, Steven Gallinger, Mark Jenkins, Kevin Sweet, Finlay Macrae, Ingrid Winship, Susan Parry, Daniel Buchanan. Envoi Pathology, Brisbane, Australia; University of Melbourne, Melbourne, Australia; University of Toronto, Toronto, Canada; Ohio State University Medical Centre, Colombus, OH; New Zealand Familial Gastrointestinal Cancer Service, Auckland, New Zealand.

Background: Serrated polyposis (SP) is a disorder characterised by the presence of multiple serrated polyps in the large intestine and an increased risk of colorectal cancer. Arbitrary clinical criteria based on the number and the size of serrated polyps are currently used to define this condition. Familial clustering, restriction to Northern European ancestry and increased risk of colorectal cancer in relatives of affected individuals suggest an inherited syndrome with genetic alteration. Recent studies have reported germline pathogenic variants in rare affected families. However, no single gene has been identified as the cause of SP.

Design: The Genetics of Colorectal Polyposis Study recruited 422 families with at least one SP-affected individual from Family Cancer Clinics within Australasia and North America. We selected a subset of individuals for genetic screening based on clinical criteria (young age at diagnosis, high polyp burden, familial clustering). Whole exome sequencing was performed in 58 individuals and whole genome sequencing in 16 . Variants were prioritised based on a frequency of $<0.1 \%$ in reference databases and as predicted to be damaging according to at least 2 out of 4 in silico programs.

Results: The screened SP population consisted of 74 individuals ( $68 \%$ female), with mean age at diagnosis of 45.5 years, mean serrated polyp number of 47 (range 5-130), with a first-degree relative affected with SP in $22.2 \%$ or with colorectal cancer in $37.5 \%$. No likely deleterious germline variant was identified in known hereditary colorectal genes (APC, BMPR1A, CDH1, CHEK2, EPCAM, GREM1, MLH1, MSH2, MSH6, MUTYH, PMS2, POLD1, POLE, PTEN, SMAD4, STK11, TP53) or in any recently reported candidate genes that regulate oncogene induced senescence (ATM, PIFI, $T E L O 2, X A F 1$, and $R B L 1$ )

Conclusions: No genetic cause for SP was associated with established colorectal cancer predisposition genes from a large scale genetic screen in a selected population with strong phenotype. In addition to our recent report showing lack of RNF43 mutation in 221 SP individuals, we did not find any mutation in other reported candidate genes.

\section{Somatic Genetic Alterations in Mismatch Repair Deficient} Colorectal Carcinomas Not Associated with Lynch Syndrome

Christophe Rosty, Mark Clendenning, Aung K Win, Finlay Macrae, Ingrid Winship, John Hopper, Mark Jenkins, Dallas English, Daniel Buchanan. Envoi Pathology, Brisbane, Australia; University of Melbourne, Melbourne, Australia.

Background: Mismatch repair (MMR) protein deficiency is the hallmark of colorectal carcinoma (CRC) in Lynch syndrome patients and sporadic CRC with MLHI methylation. Patients with an MMR-deficient CRC who have no deleterious germline mutation in a DNA MMR gene, and with absence of tumor $M L H 1$ methylation or $B R A F^{V 600 E}$ mutation if the tumor is $M L H 1$-deficient, are grouped under the category of Lynch-like syndrome. Biallelic somatic alterations in a DNA MMR gene are one possible cause for MMR deficiency in Lynch-like syndrome.

Design: CRC cases from two large population-based cohorts have been tested for MMR protein deficiency and $M L H 1$ methylation for MLH1-deficient tumors. Tumors from Lynch-like syndrome patients were selected for somatic mutation screening in DNA MMR genes using Custom AmpliSeq panel and sequencing, and for loss of heterozygosity (LOH) using Affymetrix Oncoscan array.

Results: From an initial cohort of 1639 CRC patients tested for MMR protein deficiency, 63 Lynch-like syndrome patients were identified and 50 tumors were successfully tested. Biallelic somatic alterations were identified in 18 cases (36\%), a single somatic alteration in 24 cases $(48 \%)$, and no somatic alteration in 7 cases $(14 \%)$. The proportion of CRC with biallelic somatic alterations was higher for MSH2-deficient tumors $(5 / 10,50 \%)$ and MLH1-deficient tumors (12/31,39\%), compared with MSH6-deficient tumors (1/6, $17 \%)$ and PMS2-deficient tumours (0/3).

Conclusions: Biallelic somatic alterations in DNA MMR genes account for a significant proportion of Lynch-like syndrome patients and, when present, exclude Lynch syndrome. However, the majority of Lynch-like syndrome cases remain unexplained. In particular, a single somatic alteration can be associated with a missed second hit that can be either somatic or germline, therefore not excluding Lynch syndrome. 
793

Yield of Universal Testing for Mismatch Repair Protein Deficiency in 2077 Colorectal Carcinomas

Christophe Rosty, Gregory Miller, Mark Bettington, Ian Brown. Envoi Pathology, Brisbane, Australia; University of Queensland, Brisbane, Australia.

Background: Lynch syndrome is the most common cause of inherited colorecta carcinoma (CRC). Current surveillance guidelines are effective at decreasing the morbidity and mortality of Lynch syndrome-associated cancers; however many cases are still missed. Among various screening strategies, testing all newly diagnosed CRCs for MMR protein deficiency, known as universal testing, has recently emerged as the preferred approach to identify potential Lynch syndrome individuals, who then may require additional testing and genetic counselling.

Design: All newly diagnosed CRCs from initial biopsy or surgical resection specimen were screened for MMR protein expression by immunohistochemistry. A 2-step approach was used: PMS2 and MSH6 testing followed by the testing of the respective MMR protein partner if one of the proteins was lost. We retrospectively searched our pathology database for MMR protein expression results across a 5-year period (20122016) during which universal testing was performed. Clinical and pathological data were extracted from the pathology report.

Results: 2077 consecutive CRCs were tested for MMR protein expression. Mean age at diagnosis was 68.4 years (range 18-96). MMR protein deficiency was identified in 404 cases $(19.5 \%)$. The detailed results of the abnormal immunohistochemical patterns are displayed in the table, stratified by age groups.

\begin{tabular}{|l|c|c|c|c|c|}
\hline $\begin{array}{l}\text { MMR protein } \\
\text { loss }\end{array}$ & $\begin{array}{c}\text { All } \\
\text { agesn=2077 }\end{array}$ & $\begin{array}{c}<50 \\
\text { yearsn=191 }\end{array}$ & $\begin{array}{c}50-59 \\
\text { yearsn=289 }\end{array}$ & $\begin{array}{c}60-69 \\
\text { yearsn=535 }\end{array}$ & $\begin{array}{c}>70 \\
\text { yearsn=1062 }\end{array}$ \\
\hline MLH1/PMS2 & 364 & 13 & 11 & 35 & 305 \\
\hline MSH2/MSH6 & 21 & 1 & 1 & 7 & 12 \\
\hline MSH6 & 12 & 3 & 3 & 4 & 2 \\
\hline PMS2 & 7 & 2 & 0 & 2 & 3 \\
\hline
\end{tabular}

The vast majority of CRCs with MLH1/PMS2 loss were diagnosed in patients older than 70 years $(83.8 \%)$, most of them are likely to be secondary to sporadic $M L H 1$ methylation. In 8 cases with MLH1/PMS2 loss, loss of MSH6 expression was also present. MMR protein deficiency patterns suggestive of a defect in MSH2, MSH6 or PMS2 comprised 40 cases, of which 34 were found in individuals aged 50 years or older. CRCs with MSH2/MSH6 loss were most commonly found in patients older than 70 years $(57 \%)$. Conclusions: Universal testing for MMR protein deficiency in CRC identifies abnormal patterns of expression suggestive of Lynch syndrome in all age groups, including many in those excluded by current guidelines. Further studies are needed to demonstrate the actual rate of Lynch syndrome individuals identified from this initial screening.

794 Interobserver Variability of Histopathological Regression Scoring Systems Following Neoadjuvant Chemo-Radiotherapy in Esophageal Cancer

Paromita Roy, Sayali N Honap, Indu Arun, Robin Thambudorai, Indranil Mallick. Tata Medical Center, Kolkata, West Bengal, India.

Background: Neoadjuvant chemoradiotherapy (NACRT) is the treatment of choice in operable esophageal carcinoma (EC), with significant improvement in survival compared to surgery alone. Though tumour regression grade (TRG) is well-documented as a prognostic indicator, consistent reporting remains a challenge. The aim of this study was to assess the response to treatment, and interobserver concordance using 6 common TRG systems (Table 1).

Design: We reviewed the histological parameters of consecutive cases of EC who completed NACRT followed by esophagectomy at our center. All patients were treated with radiotherapy and concurrent paclitaxel-carboplatin based chemotherapy (CROSS protocol). Regression scoring using the 6 different systems was done by 2 independent observers.

Results: 38 patients (35 Squamous carcinomas, 2 Adenocarcinoma, NOS, 1 Adenosquamous) showed median age of 56.5 years and predominantly mid-esophageal location (22/38). Majority had cT3 tumours (20/38). Most tumours showed some degree of regression, with $34.2 \%$ patients showing complete pathological response as per CROSS TRG and $39.4 \%$ as per the other systems. Commonest regression changes were dense chronic inflammation, fibrosis and keratin granulomas. Interobserver agreement in the TRGs for the 6 systems compared are shown in table1. The CROSS system had almost perfect agreement (kappa $>0.81$ ). The other percentage based systems (Swisher and RCPath) also had substantial agreement (kappa 0.61 to 0.8 )). The Japanese society TRG had moderate agreement. The College of American Pathologists(CAP) recommended modified Ryan's TRG showed substantial agreement (kappa0.67), while the five-tiered Mandard TRG, based on the relative degree of fibrosis to residual tumour, showed the lowest score

\begin{tabular}{|l|l|l|l|}
\hline Tumour Regression Grading system & kappa (k) & $95 \%$ CI & p value \\
\hline Modified Ryan & 0.67 & 0.49 to 0.84 & $<0.001$ \\
\hline Swisher & 0.69 & 0.50 to 0.87 & $<0.001$ \\
\hline Japanese Society & 0.55 & 0.37 to 0.73 & $<0.001$ \\
\hline Royal College of Pathologists & 0.61 & 0.41 to 0.81 & $<0.001$ \\
\hline Mandard & 0.56 & 0.38 to 0.74 & $<0.001$ \\
\hline Cross study TRG & 0.82 & 0.67 to 0.96 & $<0.001$ \\
\hline
\end{tabular}

Conclusions: Though the widely used Ryan TRG showed substantial concordance, the CROSS TRG score showed the best interobserver concordance and addressed the response in lymph nodes, and should be considered for widespread adoption.
Macrophage Aggregation Is a Salient Feature of Acute Rejection in Small Intestinal Allografts

Natalia Rush, Chandrashekhar Kubal, Romil Saxena. Indiana University School of Medicine, Indianapolis, IN.

Background: Small intestinal transplantation is an accepted therapeutic modality for end-stage intestinal failure. The aim of this study was to characterize the inflammatory infiltrate in the lamina propria of small intestinal allografts undergoing rejection.

Design: We retrieved biopsies with a diagnosis of acute rejection from the pathology database. Biopsies of native and allografted small intestine with no pathologic change served as controls. Immunohistochemical stains for CD4, CD8 and CD163 were performed. Each marker was evaluated as a percentage of the total number of inflammatory cells in the lamina propria.

Results: The percentages of CD4 and CD8 cells were not increased in small intestina allograft biopsies. Percentage of CD8 positive cells was decreased in some allografts, attributable to immunosuppressive medications.

The percentage of CD163 positive cells was increased in the allografts. These macrophages were present as aggregates in $82 \%(14 / 17)$ of the allograft biopsies. Macrophage aggregates were also seen in one of five control biopsies. This biopsy was from a small intestinal allograft and showed no pathologic change on hematoxylin-eosin. The subsequent biopsy from this patient showed severe rejection.

\begin{tabular}{|l|l|l|}
\hline Percentage of cells & Rejection $(\mathrm{n}=17)$ & Controls $(\mathrm{n}=5)$ \\
\hline CD4 (median, range) & $5 ; 1-20$ & $5 ; 5-20$ \\
\hline CD8 (median, range) & $10 ; 2-30$ & $5 ; 5-10$ \\
\hline CD163 (median, range) & $50 ; 10-75$ & $20 ; 20-20$ \\
\hline
\end{tabular}

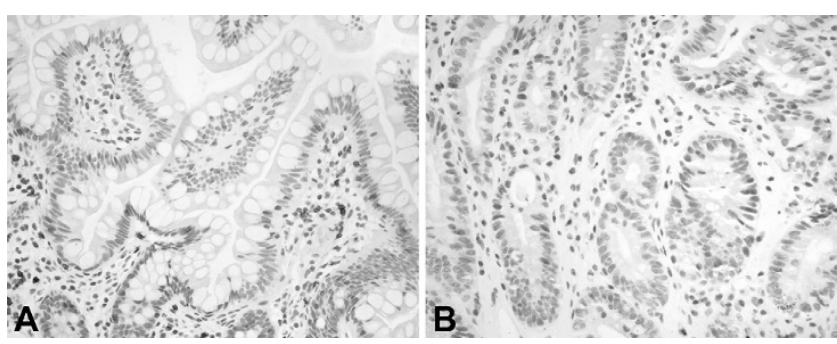

CD8 in non-rejecting allograft (A) and in severe rejection without ulceration (B)

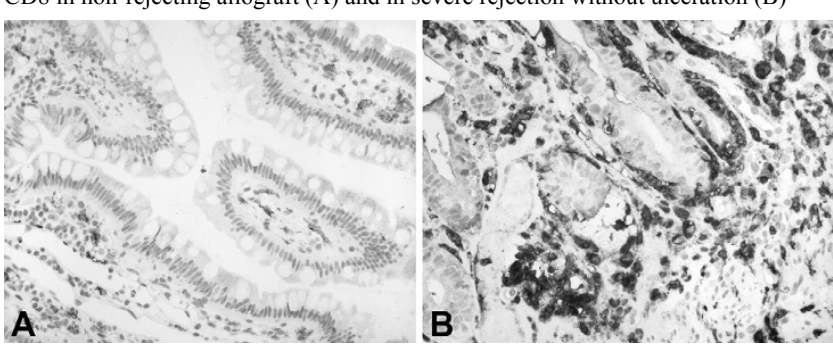

CD163 in non-rejecting allograft (A) and in severe rejection without ulceration (B)

Conclusions: Macrophage infiltration and aggregation is a salient feature of acute rejection in small intestinal allografts.

Macrophage infiltration and aggregation might be an early feature of acute rejection. In this series, it was present in a biopsy with no pathologic features of rejection before features of rejection became apparent in a subsequent biopsy.

796 Multinucleated Giant Cells in the Gastroesophageal Junction and Gastric Mucosa

Taha Sachak, Wendy L Frankel, Christina A Arnold, Wei Chen. The Ohio State University Wexner Medical Center, Columbus, $\mathrm{OH}$.

Background: In our clinical practice, we occasionally encounter multinucleated giant cells (MGCs) in the gastroesophageal junction (GEJ) and gastric mucosa. MGCs contain hyperchromatic, overlapping nuclei, and variable cytoplasm. Little is known about the origin and significance of these cells. Our goal is to identify the origin of MGCs using immunohistochemistry, determine their clinicopathological associations, and therefore improve diagnostic accuracy and avoid pitfalls.

Design: 352 contiguous biopsies and 1 resection specimen from stomach and GEJ were identified from archives in January 2016. Patient age, sex, clinical indication for endoscopy, and pathologic diagnosis were noted. The number of MGCs per 10 high power fields (HPF) was recorded. $17 \mathrm{MGC}$ cases (6 stomach; 11 GEJ) were immunostained for smooth muscle actin (SMA), desmin, CD117, S100, cytokeratin AE1/3, chromogranin, and CD68.

Results: MGCs were identified in 22 cases ( 7 gastric, 15 GEJ; $5.6 \%$ ). Patients' average age is 53 years. Male:female ratio is $1.2: 1$. Most cases (64\%) have only 1 or 2 MGCs per $10 \mathrm{HPF}$. MGCs are located in the lamina propria of the gastric/GEJ mucosa, with an accentuation in the subepithelial zone. The median number of nuclei in a MGC is 5 (ranging from 3 to 16). The nuclei are touching/overlapping, often arranged into "wreath" or "caterpillar" configurations. Cytoplasm is variable from scant to moderate. MGCs appear to be in continuation with the upward stranding muscularis mucosae in some areas. MGCs are positive for SMA, desmin, while negative for cytokeratin AE1/3, CD68, S100, chromogranin, and CD117. Most common clinical history is epigastric pain, gastroesophageal reflux, and Barrett esophagus. Most common associated pathologic diagnosis is reactive (chemical) gastropathy (71\% gastric biopsies) and gastroesophageal reflux (73\% GEJ specimens). 


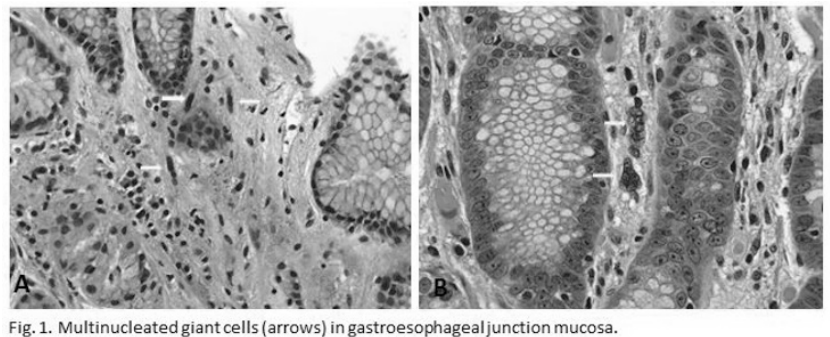

Conclusions: We conclude that MGCs in the gastric and GEJ mucosa represent atrophic smooth muscle cells originated from muscularis mucosae, as part of reactive stromal changes that are often associated with gastroesophageal reflux and reactive (chemical) gastropathy. Awareness of MGCs is important to avoid erroneous diagnoses such as carcinoma, neuroendocrine hyperplasia, or viral cytopathic effects in daily GI sign out.

797 Difference of Histological Alteration Between Preoperative Chemoradiotherapy and Chemotherapy, and It's Clinical Implication

Naoki Sakuyama, Motohiro Kojima, Shingo Kawano, Norio Saito, Masaaki Ito, Atsushi Ochiai. National Cancer Center Hospital East, Kashiwa, Chiba, Japan.

Background: The aim of the study was to elucidate difference in the histological features and clinical outcome between preoperative chemoradiotherapy and chemotherapy in patients with rectal cancer.

Design: One-hundred-thity-eight rectal cancer patients who underwent sphincter saving operations entered into this study. Of these, 44 patients received preoperative chemoradiotherapy (CRT group), 44 patients received preoperative chemotherapy (CT group), and 50 patients underwent surgery alone (SA group). Differences of histological features, clinicopathological features and clinical outcomes were compared among three groups. H.E slide from the maximum tumor slice were obtained, and area of residual tumors (ART) was determined using morphometric software.

Results: Histological features of the tumor after CRT and CT groups are much different. Case with CRT group showed more marked fibrosis than case with CT and SA group Number of immune cells was significantly less in CRT group than other groups. ART was significantly smaller in CRT group than other groups. In particular, total area of residual tumor, residual tumor area within muscular layer, and that beyond the muscular layer were significantly smaller in CRT group than CT group, and SA group. On the other hand, in CRM negative pStageI-III cases, clinical outcome were not statistically different between CRT and CT groups. Although Dworak regression grade and fibrotic change did not, ART and ypTNM classification was associated with DFS both in preoperative $\mathrm{CRT}$ and $\mathrm{CT}$ groups.

Conclusions: Different histological features after CRT and CT were elucidated. Regression grade based on the data of ART may provide useful prognostic information regardless of the variation of preoperative therapy.
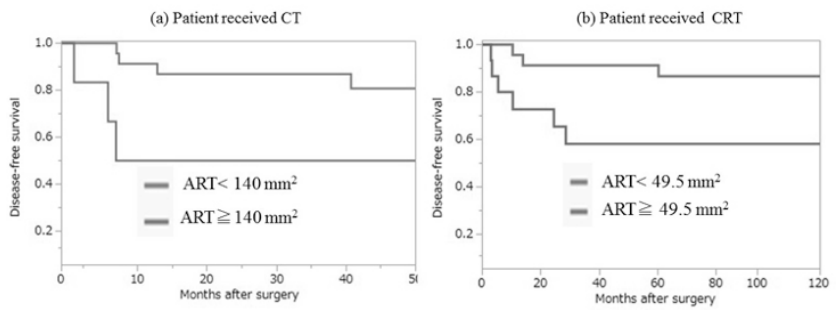
Figure 1: Disease-free survival curve for the impact of area of residual tumor (ART) on outcome in rectal
cancer patients receiving CT and CRT in CRM negative pStage I-III cases.

(a) CT (ART; P $=0.015$ ); (b) CRT(ART; P $=0.028$ )

798 Definition of Barrett Esophagus in the United States (US): Support for Retention of a Requirement for Goblet Cells

Kevan Salimian, Kevin Waters, Ogechukwu Pearl Eze, Maryam Pezhouh, Yaman Tarabishy, Lysandra Voltaggio, Elizabeth A Montgomery. Johns Hopkins Hospital, Baltimore, MD.

Background: Barrett esophagus (BE) is a premalignant state for which endoscopic surveillance is currently recommended. However, the global definition and criteria for $\mathrm{BE}$ are controversial. Historically, $\mathrm{BE}$ has been histologically defined as having columnar-lined mucosa (CLM) in the tubular esophagus. Many studies suggest a strong link between intestinal metaplasia (IM) within the CLM and the development of esophageal adenocarcinoma (EAC). As such, pathologists in Europe and the US require IM to diagnose BE. However, IM is not a diagnostic requirement for pathologists in the UK and Japan. Some data based on European patients argue that IM accompanies less than $50 \%$ of early esophageal EACs, leading to a push for worldwide unification of the definition of BE, whereby only CLM would be required. We devised the current study to determine whether IM accompanies EAC in a US patient cohort in an effort to aid in establishing an appropriate definition for BE.

Design: We examined a series of 139 consecutive endoscopic mucosal resections or esophagectomies for EACs performed at a US tertiary care center from January 2011 to June 2016. Patient demographics and clinical history were collected and the presence or absence of CLM with IM was evaluated by multiple pathologists.
Results: The patients were stratified based on the presence of IM (IM+) or absence of IM (IM-) within CLM. Ninety-seven (70\%) patients were IM+, while $42(30 \%)$ patients were IM-. We postulated that treatment-related changes as a result of prior chemotherapy or radiation might preclude our ability to detect pre-existing IM. When we restricted our analysis to patients who had not been treated prior to resection, only 5 out of 39 $(15 \%)$ were IM-. Tumors found in IM- patients tended to be more aggressive and were at an advanced pT stage at the time of resection $(57 \%$ pT3 or greater, IM-; $31 \% \mathrm{pT} 3$ or greater, IM+; $\mathrm{p}=0.02)$ such that the tumor may have "overgrown" zones of IM. The average age (65, IM+; 67, IM-), gender ( $90 \%$ male, IM+; $83 \%$ male, IM-) and body mass index $(29 \pm 6, \mathrm{IM}+; 27 \pm 6$, IM-) was similar between each group.

Conclusions: In our US hospital population, CLM with IM in the tubular esophagus is found in association with EAC in $70-85 \%$ of patients and is less frequently detected in high stage tumors, probably reflecting "overgrowth" of the IM. While it might be reasonable to define BE in Europe and Asia as simply CLM in the tubular esophagus, we believe that based on these data the US definition of BE should continue to require the presence of IM.

799 Programmed Cell Death Ligand -1 (PD-L1) Expression in Gastrointestinal Stromal Tumors Correlates Significantly with Presence of Tumor Infiltrating Lymphocytes

Deepika Savant, Taisia Vitkovski, Rebecca Thomas, Arvind Rishi. Hofstra-Northwell Health School of Medicine, Lake Success, NY.

Background: Programmed cell death ligand-1 (PD-L1) expression has been shown to enhance efficacy of Imatinib against gastrointestinal stromal tumors (GIST). GIST show variable degrees of tumor infiltrating lymphocyte(s) (TIL). Few studies address the quantitative correlation of TIL in GIST with PD-L1 expression by immunohistochemistry (IHC). We report a correlation of PD-L1 IHC expression relative to TIL, and other clinicopathologic findings.

Design: Eight-three cases of GIST were studied for age, sex, site, T stage, histologic type, risk assessment, TIL and PD-L1 IHC expression (clone SP263; with appropriate controls). PD-L1 IHC was interpreted as negative $(0-<1 \%)$ and positive. Positive PD-L1 expression was further graded: $1+(1-10 \%), 2+(11-20 \%), 3+(21-39 \%)$ and $4+(>40 \%)$ TIL density was graded on one high power field (HPF) with highest concentration: $0=$ no TIL , low TIL (grades $1=1-5 / \mathrm{HPF}, 2=6-10 / \mathrm{HPF}$ ) and high TIL (grades $3=11-15$ / $\mathrm{HPF}, 4=16-20 / \mathrm{HPF}$ and $5=>20 / \mathrm{HPF}$ ). Correlation was performed between the PD-L1 expression, TIL and clinicopathologic findings.

Results: The mean age was 69.4 years (range $40-94$ years). Varying degrees of TIL were identified in a majority of cases $(80 / 83 ; 96.38 \%)$. Low and high TIL grades were seen in $34(40.96 \%)$ and $46(55.42 \%)$ cases respectively. TIL were distributed diffusely in $76(91.56 \%)$ and as focal aggregates in $4(4.81 \%)$ cases. Overall positive PD-L1 was identified in $48(57.83 \%)$ cases. Distribution of PD-L1 expression by cases was 1+ (19; $22.89 \%), 2+(5 ; 6.02 \%), 3+(6 ; 7.22 \%)$, and $4+(18 ; 21.68 \%)$. Within low TIL group, negative PD-L1 expression was seen in $25 / 34(73.52 \%)$ cases. In contrast, only $7 / 46$ $(15.21 \%)$ cases showed negative PD-L1 in high TIL group. None of the cases without TIL showed PD-L1 expression. There was significant correlation between PD-L1 IHC expression and TIL $(p<0.0001)$. Younger patients had higher expression of PD-L1 ( $=0.0310)$. Similarly, lower (pT) stage tumors had higher PD-L1 expression. However, neither of these showed statistical significance. There was no significant correlation of PD-L1 expression with site, size, progression risk, and sex distribution. TIL were negative for PD-L1.

Conclusions: PD-L1 IHC expression in GIST correlates significantly with the presence of higher TIL. Higher PD-L1 expression is also noted in younger patients and with lower (pT) stage. Our findings suggest using TIL quantification as a surrogate marker for PD-L1 expression, which may help stratify these patients for immunotherapy.

800 Radio-Pathologic Correlation on CT Scan Findings for Appendiceal Tumors: Width of the Appendix the Most Important Clinching Factor

Deepika Savant, Cristina Sison, Sharon X Liang, Cathy Fan. Hofstra-Northwell Health School of Medicine, Lake Success, NY

Background: Radiological findings play an important role in guiding the hand of a pathologist. This study aims to highlight the accuracy and hence the dependability on CT scan reports for appendiceal tumors.

Design: A retrospective study of the appendiceal lesions was done for the past 3 years based on histopathology reports. The cases included in the study were primary appendiceal tumors which had preoperative CT scan reports. Metastatic tumors of the appendix were excluded from the study. The cases were studied with respect to age, sex, appendiceal length, width, thickness, volume, different types of tumor, tumor size, site, TNM stage, type of surgery performed and clinical findings. Fisher's exact test was used to examine associations between categorical variables. Mann-Whitney test was used to compare continuous variables between groups. Sensitivity, Specificity and predictive values and their corresponding $95 \%$ confidence intervals were calculated. Results: Total number of 53 neoplasms were included in the study. Age of patients ranged from $18-89$ years (mean 57.7) among 37 females and 16 males. Majority of the tumors were less than $10 \mathrm{~cm}$, diffusely involving the appendix; underwent appendectomy $(\mathrm{n}=40)$, right hemicolectomy $(\mathrm{n}=7)$, cecectomy $(\mathrm{n}=6)$. Incidental lesions were seen in $18.87 \%$. On correlating with the CT scan reports, $43.39 \%$ ( $n=23)$ cases did not concur with the radiology findings, whereas $56.6 \%(\mathrm{n}=30)$ concurred with the CT findings. Overall sensitivity $=50.94 \%$; $95 \%$ C.I. $(36.8-64.9)$, specificity $=16.67 \%$; $95 \%$ C.I. $(2.09-48.41)$, accuracy $=44.62 \%$; $95 \%$ C.I. $(3.22-57.47)$. Gross width was significantly higher in group whose CT scan and Pathology reports concurred than the group whose CT scan and Pathology reports did not concur and was found to be statistically significant. $(\mathrm{p}<0.009)$. There was no significant correlation between length, 
thickness, volume, tumor size, site, TNM stage, type of surgery performed and clinical findings. The sensitivity, specificity and accuracy of each type of tumor was assessed, but were found to have low precision due to limited sample size.

Conclusions: Gross width is the most important factor in clinching the diagnosis in radiological findings. However, for other apppendiceal lesions which do not distend the appendix, histopathology is still the most important tool for diagnosis. This study will be done using a larger cohort for validation of the results.

801 Antibody Mediated Rejection in Intestinal Transplant: A Correlation of Histology with Donor Specific Antibodies

Jason V Scapa, Elaine Y Cheng, Bita V Naini. David Geffen School of Medicine at UCLA, Los Angeles, CA.

Background: Intestinal transplantation (ITx) is a life-saving treatment option for selected patients with intestinal failure. However, graft failure remains high compared to other solid organ transplants with allograft rejection presumably by T-cell mediated process (acute cellular rejection (ACR)) as the leading cause. While antibody-mediated rejection (AMR) in the presence of donor specific antibodies (DSA) is a recognized complication in other solid organ grafts, the significance of DSA and AMR in ITx is largely unknown. This study aims to investigate DSA in ITx and find histologic correlates that may help in elucidating the role of AMR in ITx.

Design: We retrospectively examined 21 biopsies and 1 resection ITx specimen from 2015-16 and categorized them according to DSA status and graft dysfunction (GD). ITx occurred from 2003-2016. Cases were randomly selected from four different categories: 1) DSA present with no GD (DSA+GD-) 2) DSA present with GD (DSA+GD+), 3) DSA absent with GD (DSA-GD+), and 4) DSA absent with no GD (DSA-GD-). A comprehensive histologic exam was performed blinded to the DSA and graft status. Results: 22 cases were evaluated from 16 patients: 5 DSA+GD-, 9 DSA+GD+, 3 DSA-GD-, and 5 DSA-GD-. All cases had DSA testing within 5 months, with 16/22 within a month of the specimens. Among DSA+ patients, antibodies to HLA class II DQ loci were present in 5/5 GD+ and 2/5 GD- patients, with DSA against DQ8 seen in $3 / 4 \mathrm{GD}+$ compared to $0 / 5 \mathrm{GD}$ - patients. Normal histology was seen in 5/5 DSA+GD-, 1/9 DSA+GD+, 0/3 DSA-GD+, and 5/5 DSA-GD- cases. Histopathologic abnormalities were observed in $57 \% \mathrm{DSA}+$ with the most common findings being features of ACR such as increased crypt apoptosis $(8 / 14,57 \%)$, crypt dropout $(7 / 14,50 \%)$ and ulceration $(5 / 14,35 \%)$. There was no difference in severity of ACR in the presence of DSA. Conclusions: The presence of DSA has been shown to correlate with clinical graft dysfunction, however there does not appear to be any characteristic histologic findings. Similar to liver transplant, HLA class II DSA appears more likely to be pathologic, particularly with antibodies against the HLA-DQ locus. When histopathologic abnormalities are observed in the setting of DSA, they are those currently defined as features of ACR, making it a challenging task to establish histologic criteria for AMR in ITx. This also suggests the possibility of simultaneous occurrence of ACR and AMR in the setting of pathologic DSA, similar to what has been observed in liver transplant.

802 Gene Profiling of Precursor Serrated Polyps and Associated Carcinoma Using Next Next Generation Sequencing

Stefano Serra, Matthew Seto, Tong Zhang, Sangeetha N Kalimuthu, Suzanne KamelReid, Runjan Chetty. University Health Network, University of Toronto, Toronto, ON, Canada.

Background: The serrated neoplasia pathway accounts for $30 \%$ of colorectal cancers and is initiated by $B R A F$ activating mutations with 3 serrated precursor polyps involved: hyperplastic polyp, sessile serrated adenoma (SSA) and traditional serrated adenoma (TSA). The aim of the study was to investigate serrated precursor polyps and associated adenocarcinomas by Next Generation Sequencing (NGS).

Design: Seven precursor polyps (2 SSA and 5 TSA) and 2 TSA with an associated carcinoma were examined. Tissue was macrodissected from the precursor polyps and invasive carcinoma components using unstained, formalin-fixed, paraffin-embedded sections. DNA ( $\geq 250 \mathrm{ng}$ ) was extracted using Qiagen's QIAMP DNA Micro kit. DNA samples were run on panel with complete exonic sequence for 555 genes as well as intronic and exonic sequence for select genes known to be commonly involved in translocations. Sequencing was performed using the Illumina NextSeq 550 sequencer. NGS data was processed using the UHN-Clinical Laboratory Genetics custom bioinformatics pipeline, and filtered through the Cartagenia Bench Lab NGS (v4.2) software.

Results: See table1.

\begin{tabular}{|l|l|l|l|l|l|}
\hline Case \# & Age & Sex & Precursor lesion & \# of variants & Carcinoma \\
\hline \multirow{2}{*}{1} & \multirow{2}{*}{83} & \multirow{2}{*}{ F } & SSA + LGD & 68 & \multirow{2}{*}{ no } \\
\cline { 4 - 6 } & & & SSA & 3 & \\
\hline 2 & 40 & M & TSA & 6 & no \\
\hline 3 & 64 & F & TSA & 34 & no \\
\hline 4 & 75 & M & TSA +carcinoma & 94 & yes \\
\hline \multirow{2}{*}{5} & \multirow{2}{*}{59} & \multirow{2}{*}{ M } & TSA +carcinoma & 11 & \multirow{2}{*}{ yes } \\
\cline { 4 - 5 } & & & TSA & 11 & \multirow{2}{*}{} \\
\hline
\end{tabular}

Variants in genes occurring in both TSAs and the associated cancers showed: $B R A F$ $V 600 E$ in the precursor lesion, and variants in BRAF, PIK3CA, PRDM1, TAFIL, CDH23, RNF43, SMAD4, STK11, CHEK2, KDM6A in the invasive adenocarcinoma. $B R A F$ V600E was the only mutation seen in all samples across all cases.The $A P C$ $T 1556 \mathrm{Nfs} * 3$ mutation was present in 3 out of $5 \mathrm{TSAs}$, one of which was associated with invasive carcinoma. Three cases had RNF43 variants seen in 2 TSAs (2 different RNF43 mutations).
Conclusions: This preliminary study using NGS is the first to demonstrate a marked increase in the number of genes altered between the precursor polyps, dysplasia and adenocarcinoma. $B R A F V 600 E$ is the only variant seen consistently in precursor lesions and associated dysplasia or invasive adenocarcinoma at high allele frequencies. The same APC gene variant, $T 1556 \mathrm{Nfs} * 3$, is seen in 3 TSAs. Genetic alteration in $R N F 43$, a component of the Wnt pathway are seen in 2 of 5 TSAs. APC and RNF43 gene variants may result in perturbation of the Wnt pathway and play an important role in the pathogenesis of these lesions.

803 Crospovidone and Microcrystalline Cellulose: A Novel Description of Pharmaceutical Fillers in the Gastrointestinal Tract Sophia M Shaddy, Michael A Arnold, Konstantin Shilo, Wendy L Frankel, Alan Harzman, Peter Stanich, Martha Yearsley, Christina A Arnold. The Ohio State University Wexner Medical Center, Columbus, OH; Nationwide Children's Hospital, Columbus, OH.

Background: Pharmaceutical fillers are inactive substances incorporated into medications to facilitate drug delivery. We report the first study of the fillers crospovidone and microcrystalline cellulose (MCC) in the gastrointestinal tract (GIT). Design: We examined 503 in-house GIT cases from 261 patients between 09/11/2015$10 / 23 / 2015$, and identified 29 cases of crospovidone and or MCC from 26 patients based on previously described morphology at other sites. The control group consisted of an equal number of site matched specimens. One case was subject to special stains (Table 1). To confirm the histologic diagnosis, a variety of purified fillers and medication tablets were histologically processed.

Results: The fillers were found in $10 \%$ of patients, and there were no specific clinicopathologic associations. Among all study specimens, the fillers were most common in the colon (colon $=58.6 \%, \mathrm{SI}=20.6 \%$, esophagus $=10.3 \%$, stomach $=10.3 \%$ ). On H\&E, crospovidone is nonbirefringent and has a coral shape with a pink core and purple coat; MCC is clear and brightly birefringent with a matchstick shape. Identical material was seen in the processed crospovidone and MCC powder and oxycodoneacetaminophen and omeprazole tablets.

Conclusions: In summary, crospovidone and MCC are common, biologically inert substances most often seen in the colon. Their presence outside of the bowel may serve as a marker of perforation. Awareness is also important to distinguish them from parasites, calcifications, and other medications. We report a unique histochemical profile as a helpful diagnostic aide, and caution that the fillers have slightly divergent appearances in the GIT when compared to the lung.

\begin{tabular}{|l|l|l|}
\hline \multicolumn{2}{|l|}{$\begin{array}{l}\text { Table 1: Morphologic and Histochemical Profile of Crospovidone and Microcrystalline } \\
\text { Cellulose }\end{array}$} & \\
\hline & Crospovidone & Microcrystalline Cellulose \\
\hline & Coral & Rod, Match-stick \\
\hline Shape & $\begin{array}{l}\text { Segments have pink center with } \\
\text { purple coat }\end{array}$ & Clear \\
\hline & Non-birefringent & Brightly-birefringent \\
\hline Polarized Light & No & Yes \\
\hline Refractile & Bright pink & Light blue \\
\hline Alcian Blue & Dark red-brown & Light pink \\
\hline von Kossa & Yellow center with red outline & Clear \\
\hline Mucicarmine & Yellow & Yellow \\
\hline Movat & Clear \\
\hline Fontana-Mason & Black & Light purple \\
\hline PAS/D and PAS & Grey-blue & Light blue \\
\hline AFB & Tan-pink & Brown-black \\
\hline GMS & Brown-black & $\begin{array}{l}\text { Light salmon, without apple green } \\
\text { birefringence }\end{array}$ \\
\hline Congo Red & $\begin{array}{l}\text { Dark orange, without apple green } \\
\text { birefringence }\end{array}$ & \\
\hline
\end{tabular}

804 Effects of Interobserver Variability and Subspecialty Signout on Diagnosing Microscopic Colitis

Meenal Sharma, Christa L Whitney-Miller, Michael G Drage, Aaron R Huber, Raul S Gonzalez. University of Rochester, Rochester, NY.

Background: Microscopic colitis (MC) is a diagnostic category that includes lymphocytic colitis (LC) and collagenous colitis (CC). The colon is endoscopically normal in MC; diagnosis requires microscopy. Proposed criteria include increased lamina propria cellularity and more than 20 intraepithelial lymphocytes per 100 enterocytes for LC, and subepithelial collagen thicker than $10 \mu$ for CC. Studies have shown a small degree of interobserver and intraobserver variability in diagnosing $\mathrm{MC}$, suggesting some subjectivity. This has not been evaluated in the context of subspecialty signout (SSSO) or a group consensus.

Design: We reviewed 133 colon biopsies diagnosed as LC, CC, MC, or normal but with mild changes insufficient for MC. All predated true SSSO at our institution. For each, we recorded original diagnosis and whether the signout pathologist had subspecialty training in gastrointestinal (GI) pathology. H\&E slides from all 133 were independently reviewed by 3 pathologists with GI subspecialty training, rendering diagnoses of LC, $\mathrm{CC}$, or normal. Cases lacking independent consensus by this method were reviewed by the same 3 pathologists via consensus conference to establish a diagnosis. Consensus diagnoses were compared to the originally rendered diagnoses and to the independent diagnoses of the consensus pathologists.

Results: Consensus diagnoses for the biopsies were normal $(\mathrm{n}=34), \mathrm{LC}(\mathrm{n}=57)$, and $\mathrm{CC}(\mathrm{n}=42)$. "Normal" cases were most commonly agreed upon by the 3 reviewing pathologists prior to consensus ( $27 / 34$ cases, $\mathrm{P}=0.0073$ vs. $\mathrm{LC}, \mathrm{P}=0.0172$ vs. $\mathrm{CC}$ ). 
LC was agreed upon pre-consensus in 28/57 cases, and $\mathrm{CC}$ in $22 / 42$ cases $(\mathrm{P}=0.840)$. The 3 reviewing pathologists independently agreed with $80 \%, 80 \%$, and $94 \%$ of consensus diagnoses $(\mathrm{kappa}=0.70,0.69$, and 0.91$)$. Among cases signed out by non-GI pathologists, $83 \%$ of diagnoses agreed with the consensus (kappa $=0.75,95 \% \mathrm{CI}=0.61$ 0.89 ), compared to $75 \%$ signed out by GI pathologists (kappa $=0.59,95 \% \mathrm{CI}=0.42$ 0.76). However, the two populations were dissimilar; the cases signed out by non-GI pathologists had a higher proportion of "normal" biopsies per consensus $(\mathrm{P}=0.006)$, potentially making the set easier.

Conclusions: Despite existing criteria, diagnosing LC and CC is associated with some interobserver variability. Subspecialty training alone does not necessarily increase diagnostic accuracy, as pathologist consistency and acumen can vary regardless of training. One benefit of SSSO is facilitation of a group consensus conference, which aids in diagnosis of difficult or borderline cases. This should be kept in mind when evaluating biopsy specimens for MC.

805 Expression of Epithelial-Mesenchymal Transition Markers E-Cadherin and Vimentin in Esophageal Adenocarcinoma and Squamous Cell Carcinoma and Significant Association with New Proliferation Markers MCM4 and MCM7

Meenal Sharma, Amy Lalonde, Dongfeng Tan, Tongtong Wu, Zhongren Zhou. URMC, Rochester, NY; MD Anderson, Houston, TX.

Background: Epithelial-mesenchymal transition (EMT) plays a key role in the invasion of carcinoma. Loss of E-cadherin and acquisition of vimentin are two critical steps in EMT. Our study evaluated their expression in esophageal adenocarcinoma (EAC) and squamous cells carcinoma (ESCC) and precancerous lesions.

Design: TMA of 110 EAC, 23 ESCC, 66 squamous epithelium (SE), 68 columnar cell metaplasia (CM), 37 Barrett's esophagus (BE), 27 low-grade dysplasia (LGD) and 11 high-grade dysplasia (HGD), were immunohistochemically stained for both E cadherin and Vimentin. E cadherin and Vimentin were scored for positive, negative and for percentage $(0-100 \%)$ of positive cells.

Results: Of 110 EAC cases, $90 \%(n=99)$ were positive for E-cadherin and $3 \%(n=3)$ were positive for vimentin. Of the $23 \mathrm{ESCC}, 100 \%(\mathrm{n}=23)$ were positive for E-cadherin and $9 \%(\mathrm{n}=2)$ positive for both E-cadherin and vimentin. In the EAC group, the mean survival time for E-cadherin positive cases was 38.8 months vs 48.3 months for E-cadherin negative cases although it was not statistically significant. The mean percentage of E-cadherin expression was significantly higher for ESCC (70.2\%) compared to EAC $(52 \%)$. No significant difference was seen between E-cadherin expression in EAC and other precancerous lesions including BE, LGD and HGD. Loss of E-cadherin in EAC correlated significantly with higher proliferation (MCM4 $=63.9 \%$ and MCM7 $=76.1 \%$ ) compared with expression of E-cadherin $(\mathrm{MCM} 4=37.7 \%$; $\mathrm{MCM} 7=42.2 \%)$. Vimentin positive EAC cases also had a significantly higher proliferation (MCM $7=87.5 \%$ ) compared with vimentin negative cases (MCM7 $=42.8 \%$ ). There was no significant relationship between either E-cadherin or vimentin expression with any clinicopathologic features.

Conclusions: EMT transition existed in low percentage of EACs and ESCC cases. A loss of $\mathrm{E}$ cadherin expression and expression of vimentin are associated with significantly increased proliferation.

806 Targeted Next-Generation Sequencing Supports Epidermoid Metaplasia of the Esophagus Is a Precursor to Esophageal Squamous Neoplasia

Aatur D Singhi, Christina A Arnold, Dora M Lam-Himlin, Marina N Nikiforova Lysandra Voltaggio, Elizabeth A Montgomery. University of Pittsburgh Medical Center, Pittsburgh, PA; Ohio State University, Columbus, OH; Mayo Clinic, Scottsdale, AZ; Johns Hopkins Hospital, Baltimore, MD.

Background: Esophageal epidermoid metaplasia (EEM) is a rare condition that involves the middle-to-distal esophagus. It is sharply demarcated and defined histologically by epithelial hyperplasia, a prominent granular cell layer and superficial hyperorthokeratosis. In addition, studies have suggested a possible association between EEM, and squamous dysplasia and esophageal squamous cell carcinoma (ESCC) However, whether EEM represents a preneoplastic lesion is unknown. In order to further characterize EEM and define its relationship to squamous neoplasia of the esophagus, we performed targeted next-generation sequencing (NGS) of specimens with EEM, adjacent high-grade squamous dysplasia (HGSD)/ESCC, and uninvolved squamous mucosa. Design: Twenty-nine esophageal specimens ( 28 biopsies and 1 resection) were collected from 14 patients. In addition to EEM, 3 patients had adjacent $\operatorname{HGSD}(\mathrm{n}=2)$ or $\operatorname{ESCC}(\mathrm{n}$ $=1$ ). Uninvolved esophageal squamous mucosa was available for 10 patients. Targeted NGS was performed using the Ion Torrent PGM for 30 genes that are often linked to malignancy with an average depth of coverage $>1000 \mathrm{X}$. Copy number variant analysis for 24 genes was also done. Deletions in $C D K N 2 A$ were confirmed by fluorescence in situ hybridization (FISH).

Results: Targeted NGS detected genetic alterations in 9 of $14(64 \%)$ cases of EEM. The most frequently mutated genes included TP53 $(\mathrm{n}=7), P I K 3 C A(\mathrm{n}=2)$ and EGFR $(\mathrm{n}=$ 2). Allelic frequencies (AFs) for TP53 and PIK3CA mutations ranged between $3 \%$ and $8 \%$. Sequencing of adjacent HGSD and ESCC also identified mutations in TP53 $(\mathrm{n}=3)$ and PIK3CA $(\mathrm{n}=1)$ and were the same as those present within the background EEM. However, the AFs for TP53 and PIK3CA were higher and ranged between $12 \%$ and $33 \%$. Moreover, deletions in $C D K N 2 A(\mathrm{n}=2)$ were also present and confirmed by FISH. No genetic alterations were found in the uninvolved esophageal squamous mucosa. Conclusions: Genetic alterations are frequently seen in EEM and are similar to those previously reported in squamous neoplasia of the esophagus. Further, higher AFs in TP53 and PIK3CA and the acquisition of CDKN2A deletions in adjacent HGSD and ESCC suggest a clonal evolution from EEM. These findings support EEM is a precursor lesion to esophageal squamous neoplasia.
807 Genome-Wide Copy Number Aberrations Analysis Reveals Recurrent High Copy Number Gain of PTGER4 Gene in Colonic Mixed AdenoNeuroEndocrine Carcinoma and NeuroEndocrine Carcinoma

Namita Sinha, Daniel Gaston, Marissa Goudie, Makoto Matsioka, Weei-Yuan Huang. Nova Scotia Health Authority, Dalhousie University, Halifax, NS, Canada.

Background: Mixed AdenoNeuroEndocrine Carcinoma (MANEC) is an aggressive neoplasm of the colon, characterized by coexisting adenocarcinoma and neuroendocrine carcinoma components. The prognosis of MANEC is distinctly worse than conventional colonic adenocarcinoma (ADC). For a clinician, the major challenge is whether a MANEC should be managed as conventional ADC or NEC. To explore carcinogenic processes of these tumors, we analyzed genome-wide copy number aberrations (CNA) in MANEC and NEC. The results were then compared to CNA in ADC, obtained from published literature, including data from TCGA (The Cancer Genome Atlas).

Design: A total of 24 tumors (18 MANEC and 6 NEC) were identified. Genomic DNA from nineteen tumors (14 MANEC and 5 NEC) with known microsatellite instability and BRAFV600E mutation status were subjected to genome-wide CNA analysis using a molecular inversion probe single-nucleotide polymorphism assay (Affymetrix OncoScan assay). Results were analyzed using Nexus Copy Number software with a built-in statistical GISTIC (genomic identification of significant targets in cancers) algorithm. A genomic region with recurrent high copy number $(\mathrm{CN})$ gain $(4$ copies or more) was further analyzed by an interphase FISH (fluoresce in situ hybridization) assay on all 24 tumors.

Results: Recurrent CNA in MANEC were gain on chromosome (chr) 5p (10/14 cases), 20q, 13q, 7, 8q and loss on chr 1p, 4q, 5q, 8p, 18q; in NEC were gain on chr $7 \mathrm{q}, 8 \mathrm{q}, 15 \mathrm{q}$ and loss on chr 4p, 5q, 7p. Application of statistical GISTIC algorithm identified four significant regions that may drive MANEC and NEC tumorigenesis: $8 \mathrm{q} 24.2,13 \mathrm{q} 22,20 \mathrm{q} 13$ and $5 \mathrm{p} 13.1$, in which high $\mathrm{CN}$ gain of a candidate cancer driver, PTGER4 (prostaglandin E2 receptor 4) gene on 5p13.1 was identified in 4 MANECs and two NECs (6/19). Two of the tumors (one MANEC and one NEC) harbor PTGER4 gene amplification of up to 33 copies. In contrast, there was only $1 \%$ (6 out of 611) of conventional colonic adenocarcinoma in TCGA database harboring PTGER4 gene amplification. The result of FISH assay, using CEP5 and a custom-designed probe confirmed recurrent high $\mathrm{CN}$ gain of PTGER4 gene on 10 out of 24 examined tumors. Conclusions: The frequency and pattern of CNA in MANEC were similar to published $\mathrm{CNA}$ in $\mathrm{ADC}$ except for recurrent gain of entire $5 \mathrm{p}$ arm. The identification of frequent high CN gain of PTGER4 gene on 5p13.1 suggests a potential role of inflammatory stimulation by PTGER4 up-regulation in tumorgenesis of colonic MANEC and NEC

808 Deep Inflammation in Clinically Diagnosed Ulcerative Colitis (UC) Resections: How Much Is Too Much?

Sharon Song, Stuti G Shroff, Emma E Furth, Rashmi Tondon, Kristen M Stashek. University of Pennsylvania, Philadelphia, PA.

Background: Making the correct diagnosis of UC on resection is important for proper postoperative management. We examined resection specimens from patients with a clinical diagnosis of $\mathrm{UC}$ and assessed for the presence, type, and distribution of inflammation within the muscularis propria (MP) and pericolonic soft tissue (ST) and its influence on diagnosis.

Design: 51 total colectomy resections (1/15 to 6/16) in clinically diagnosed UC patients were reviewed by 2 pathologists blinded to the original diagnosis. Pertinent clinical histories, including drug therapy, were noted. The cases were diagnosed as either UC or indeterminate colitis (IC) and the extent of deep inflammation, including ST lymphoid aggregates (LA), MP LA, perivascular inflammation (PVI), deep acute inflammation (AI) and ulceration extending from mucosal surface into MP (UMP) were noted. The number of LA (defined as a collection of lymphocytes easily visible on $2.5 \mathrm{x}$ ) in the MP and ST were noted on one representative slide (RS) and subjected to statistical analysis (student's t-test).

Results: See tables 1 and 2.

\begin{tabular}{|l|c|c|c|}
\hline & UC (n=43) & IC (n=8) & $\mathrm{p}$ \\
\hline Age (avg) (range) & $41(19-66)$ & $52(22-76)$ & \\
\hline Severely active & $56 \%$ & $100 \%$ & \\
\hline Backwash ileitis & $19 \%$ & $13 \%$ & \\
\hline Dysplasia & $19 \%$ & 0 & \\
\hline Secondary findings & $2 \%$ (diversion) & $50 \%$ (tics, infections) & \\
\hline Deep inflammation & $56 \%(24 / 43)$ & $100 \%(8 / 8)$ & \\
\hline MP LA (avg/slide) (range) & $3.3 /$ RS $(0-19)$ & $11.9 /$ RS $(1-30)$ & .03 \\
\hline ST LA (avg/slide) (range) & $2.7 /$ RS (0-11) & $13.5 /$ RS (2-21) & .001 \\
\hline PVI & $56 \%(24 / 43)$ & $100 \%(8 / 8)$ & \\
\hline Deep AI & $2 \%(1 / 43)$ & $75 \%(6 / 8)$ & \\
\hline UMP & $2 \%(1 / 43)$ & $88 \%(7 / 8)$ & \\
\hline
\end{tabular}

\begin{tabular}{|l|c|c|}
\hline \multicolumn{3}{|c|}{ Deep Inflammation in Confirmed UC Cases ( $\mathrm{n}=43$ ) } \\
\hline & MP LA per RS & ST LA per RS \\
\hline Severely active & 4.6 & 3.8 \\
\hline Non-severely active & 1.6 & 1.3 \\
\hline & $\mathrm{p}=.04$ & $\mathrm{p}=.01$ \\
\hline Treated (any) & 4.6 & 4.0 \\
\hline Non-treated (any) & 4.6 & 7.0 \\
\hline & $\mathrm{p}=.99$ & $\mathrm{p}=.47$ \\
\hline Treated (biologics) & 3.4 & 2.6 \\
\hline Non-treated (biologics) & 3.1 & 2.9 \\
\hline & $\mathrm{p}=.83$ & $\mathrm{p}=.76$ \\
\hline
\end{tabular}


Conclusions: More than $50 \%$ of UC cases show deep inflammation on resection. In path confirmed UC, the number of MP LA $(p=.04)$ and ST LA $(p=.01)$ is related to the severity of the active colitis. There was no association between drug therapy, PVI and LA. Cases diagnosed as IC showed increased numbers of LA compared to UC cases, however deep AI and UMP were more likely to garner a diagnosis of IC. In summary, PVI and MP/ST LA are exceedingly common in UC resections and their presence should not prevent a diagnosis of UC.

809 Identification and Prognostic Significance of Genetic Drivers of Adenosquamous Carcinoma of Pancreas

Belinda Sun, Paris J Vail, Erik S Knudsen, Agnieszka K Witkiewicz. Banner-University Medical Center, University of Arizona, Tucson, AZ; University of Arizona, Tucson, AZ. Background: Adenosquamous carcinoma of the pancreas (ASCP) is a rare variant of pancreatic ductal adenocarcinoma (PDAC) and has particularly poor prognosis. Little has been known about the genetic features of ASCP and drivers of this unique manifestation of PDAC.

Design: A total of 112 surgically resected PDA cases including 14 ASCP cases were analyzed by whole-exome sequencing. Somatic point mutations and insertion/deletion of DNA specific to tumor were identified using the MuTect and VarScan2 algorithms resepctively. Copy number changes were determined using GISTIC. A total of $26 \mathrm{ASCP}$ cases were further analyzed for MYC amplification by Fluorescence in situ hybridization (FISH) using Vysis LSI MYC dual color 'break apart' rearrangement probe. Validation analyses was performed with the TCGA cohort. Significant impact on survival was determined by Kaplan-Meier analysis.

Results: MYC oncogene amplification, detected by whole-exome sequencing, was found in $14 \%$ of 112 PDAC and $57 \%$ of 14 ASCP. FLG1 mutation (29\%) was also significantly enriched in our ASCP cohort. In contrast genes canonically altered in PDAC (i.e. KRAS, TP53, CDKN2A, and SMAD4) were equivalently altered in ASCP (Figure 1A). The amplification of MYC oncogene was associated with poor outcome across the cohort (Figure 1B). In the validation TCGA cohort, amplification of MYC and FLG1 mutations were also frequently identified and associated with poor-outcome (Figure 2). FISH analysis of additional ASCP cases, confirmed frequent MYC amplifications and showed that $77 \%$ ( 7 of 9 cases) of PanIN lesions coexisting with ASCP have copy increase or amplification of MYC.

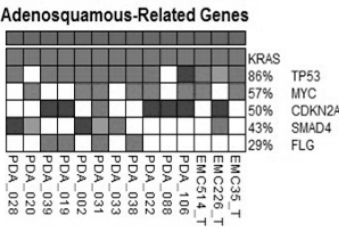

A

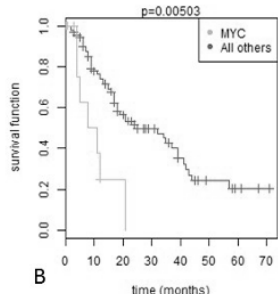

Figure 1. Integrated genetic analysis shows frequent amplification of MYC, mutation of FLG and other genes in ASCP (A). Amplification of MYC is associated with poor survival outcome in 112 cases of PDA (B).

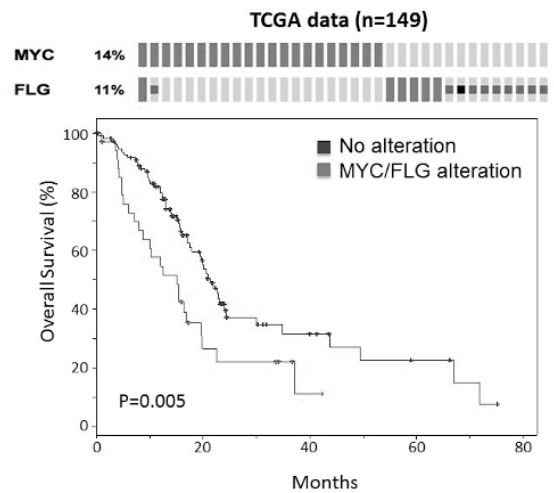

Figure 2. MYC amplification and FLG mutation are observed frequently in TCGA data and also define poor-outcome.

Conclusions: These findings suggest that specific genetic features contribute to ASCP biology and poor outcome. In particular, MYC amplification occurs relatively early as a likely driver in the oncogenesis of ASCP.

810 Structural Genomic Changes in IBD-Related Dysplasia and Preceding Copy Number Alterations in Corresponding Non-Dysplastic Mucosa

Diana Sung, Alain C Borczuk, Alina Iuga. Columbia University Medical Center, New York, NY; Weill Cornell Medical College, New York, NY.

Background: IBD-related dysplasia has a higher rate of progression and of concomitant neoplasia relative to sporadic lesions. Using Molecular Inversion Probe technology, formalin-fixed paraffin-embedded (FFPE) biopsy tissue can be processed to identify structural genetic changes using the Affymetrix ONCOSCAN for FFPE platform, allowing comparison of changes in non-dysplastic and dysplastic colonic mucosa of IBD harvested over years of endoscopic screening. Our aim was to use this platform to explore the molecular alterations of dysplasia and those that may precede its development in IBD.
Design: Seven cases with $\geq 3$ years of endoscopic surveillance and IBD-related dysplasia (6 low-grade (LGD) and 1 high-grade (HGD)) plus controls were selected. Each case included biopsies of dysplastic mucosa and concurrent non-dysplastic mucosa away from the site of dysplasia, as well as prior non-dysplastic mucosa (collected within 3 years of dysplasia) at the same site. The epithelium was microdissected and FFPE sample-derived DNA was isolated. The DNA was processed using the Affymetrix ONCOSCAN for FFPE platform and analyzed using Nexus Copy Number (BioDiscovery).

Results: Three cases of dysplasia showed significant alterations, including large segments of loss of heterozygosity $(\mathrm{LOH})$ or copy number alterations (CNA) at $5 \mathrm{q}$, $13 q, 20 p$ and $20 q$ in one case of LGD, CNA at $1 \mathrm{q}$ and $17 \mathrm{q}$ in another LGD and multiple complex alterations in HGD. In the corresponding non-dysplastic samples (both concurrent and prior) of the LGD cases, we identified short regions of CNA with the same point of origin as the long regions of $\mathrm{LOH}$ seen in dysplastic mucosa: a $0.5 \mathrm{Mb}$ region in $17 \mathrm{q}$, which included $\mathrm{BRCA} 1$, and a $0.7 \mathrm{Mb}$ and $2.3 \mathrm{Mb}$ region in $5 \mathrm{q}$. These may represent precursor genomic structural changes and were not found in controls. Conclusions: This study found small regions of CNA in non-neoplastic mucosa with the same sites of origin as large regions of LOH found in dysplasia. The discovery of these small, potentially pathogenic regions is novel. Additional studies are needed to confirm and functionally characterize these sites. FFPE biopsy samples are a useful resource in studying the progression of molecular changes that takes place in the development of IBD-related dysplasia. This can shed light on the predictive value of molecular findings in non-dysplastic mucosa and help identify IBD patients at higher risk for progression to colorectal neoplasia.

811 Type-1 Gastric Neuroendocrine Tumors; Long-Term Follow-Up and Non-Specific ER and GATA-3 Expression

Yaman Tarabishy, Ogechukwu Pearl Eze, Maryam Pezhouh, Lysandra Voltaggio, Elizabeth A Montgomery, Kevin Waters. Johns Hopkins Hospital, Baltimore, MD.

Background: Type-1 gastric well differentiated neuroendocrine (carcinoid) tumors (Type-1 gNET) are indolent, arising in the setting of ECL-cell hyperplasia due to hypergastrinemia from autoimmune metaplastic atrophic gastritis (AMAG). A recent report found that 33 of 75 Type- 1 gNETs had Ki67 proliferation indices that met criteria for grade $2(>2 \%)$, but it is not known if these tumors have worse behavior. Also, Type-1 gNETs are composed of loosely cohesive cells in the lamina propria raising the differential diagnosis of metastatic lobular breast cancer. To our knowledge, immunolabelling for breast cancer markers has not been evaluated in these tumors. We evaluated Type-1 gNETs with long-term follow-up for evidence of disease progression and immunohistochemical (IHC) staining for breast cancer markers.

Design: There were 26 Type 1 gNETs from 17 patients from our in-house surgical pathology service between 1997 and 2005. Basic demographic data were collected and patient charts were mined for evidence of metastatic disease or mortality. IHC was used to stain for Ki67, ER, and GATA-3.

Results: The mean patient age was $59,82 \%$ were female, and $65 \%$ were white. 4 tumors were from partial or total gastrectomy specimens, and 22 from biopsies. There was 4.7 years of mean follow-up per patient and no patient had a metastasis or death from disease. The Ki67 proliferation was $>2 \%$ in $15 \%$ (4/26, 95\% confidence interval (CI) $2-29 \%$ ) of tumors. Follow-up was limited in these patients (1.7 years total) with two dying of other causes within 2 years of procedure, but having imaging negative for metastases prior to death. Both ER and GATA-3 showed reactivity in Type-1 gNET. ER was at least focally positive in $35 \%$ (95\% CI $16-53 \%)$ of tumors, staining strongly in $>50 \%$ of cells in $8 \%(95 \%$ CI $0-18 \%)$ of tumors. GATA-3 was at least focally positive in $38 \%(95 \%$ CI $20-57 \%)$ of tumors, staining strongly in $>50 \%$ of cells in $15 \%(95 \%$ CI $2-29 \%$ ) of tumors.

Conclusions: In 26 Type-1 gNETs from 17 patients dating back 10-19 years, we found no metastasis or tumor related mortality. About $15 \%$ of Type- 1 gNETs has increased Ki67 proliferation rates meeting criteria for a grade 2 NET in another setting. Ki67 adds no prognostic information in this setting, but larger studies with long term follow-up are needed to confirm that these tumors are indolent regardless of the Ki67 proliferation index. Both ER and GATA-3 are reactive in a subset of these tumors and are not reliable markers to exclude metastatic breast cancer in Type 1 gNETs.

\section{Clinical and Pathologic Features of Gastrointestinal Rosai} Dorfman Disease

Yaman Tarabishy, Ogechukwu Pearl Eze, Kevin Waters, Lysandra Voltaggio, Elizabeth A Montgomery, Maryam Pezhouh. The Johns Hopkins Hospital, Baltimore, MD. Background: Rosai Dorfman disease, or sinus histiocytosis with massive lymphadenopathy, is a rare probably non-neoplastic chronic inflammatory process of uncertain etiology, differing from Langerhans cell histiocytosis and Erdheim Chester disease by its lack of $B R A \mathrm{~F}$ mutations. It is often multifocal with nodal and extranodal involvement. The extremities, trunk, and head and neck are the most commonly involved extranodal sites. Gastrointestinal (GI) involvement is uncommon, and its clinical and histologic presentation should be distinguished from more aggressive neoplastic processes such as Hodgkin and non-Hodgkin lymphomas, histiocytic sarcomas, Langerhans cell histiocytosis, and other inflammatory mesenchymal lesions. Design: We searched our Pathology Data System between the years 1984 and 2016 for Cases of Rosai Dorfman disease involving the GI tract. 14 cases were identified, of which 8 were consultation cases. Clinical, histologic, and special stain findings were reviewed. Results: The majority of patients were females $(n=11,79 \%)$. The average age at presentation was 56.7 years. Most cases were rectal $(\mathrm{n}=8,57 \%)$, followed by appendiceal $(n=2)$, small bowel $(n=2)$, right colonic $(n=1)$, and gastric $(n=1)$. Nine cases $(64 \%)$ presented as masses centered in the peri-rectal tissue or the appendiceal and small bowel mesentery. The remainder were discovered endoscopically as a polyp $(n=4)$ or a thickened mucosal fold $(n=1)$, and were centered in the mucosa/submucosa. The average size was $4.3 \mathrm{~cm}$. On histologic examination, the characteristic finding of emperipolesis 
was readily identifiable in all but one case. Storiform fibrosis was a common feature. S-100 protein immunolabeling, performed on 11 cases, was reactive in all. Examples of submitted diagnoses on the 8 consultation cases included lymphoma, sarcoma, inflammatory myofibroblastic tumor, and sclerosing mesenteritis.

Conclusions: Rosai Dorfman disease is a rare inflammatory process that can occur in the GI tract. Awareness of this very rare occurrence is important to avoid delayed diagnosis or confusion with a variety of neoplastic and non-neoplastic conditions.

813 Singapore Intestinal Metaplasia (SIM) Classification: MUC5AC Positive Immunophenotype and Multifocal Pattern Are Strong Predictors of Progression to Gastric Dysplasia

Ming Teh, Supriya Srivastava, Feng Zhu, Ting Ting Wang, Richie Soong, Khek Yu Ho, Khay Guan Yeoh, Manuel Salto-Tellez. National University Hospital, Singapore, Singapore; Cancer Science Institute, Singapore, Singapore; Queen's University, Belfast, Ireland.

Background: Intestinal metaplasia (IM) is a preneoplastic condition for gastric cancer (GC). The phenotypic pattern of mucin protein expression is altered in IM. Our aims were to identify subtypes of IM according to their pattern of mucin expression, their distribution in at risk patients of GC in Singapore and to assess the IM subtypes which were associated with progression to GC.

Design: Endoscopic biopsies were obtained from Singapore Chinese subjects aged 50 years and above ( $\mathrm{n}=2987$ ), from 2004 till 2012, systematically reviewed by expert pathologists. Biopsies of 546 cases with moderate to marked IM were stained with Haematoxylin and Eosin, HID/AB and immunohistochemistry was performed for MUC1, 2 and 5AC followed by quantitative staining analysis. Patients were followed up for a mean period of 46.6 months (range 0-88 months). Statistical analysis was performed and $\mathrm{p}$ value $<0.05$ was considered significant.

Results: 24 cases progressed to dysplasia/carcinoma (LGD, n=9; EGN, n=15) from 546 patients. MUC1 expression was observed in $24.4 \%$, MUC5AC in $43.4 \%$ and MUC2 in $100 \%$ cases. Based on HID/AB and mucin expression, type I IM was seen in $55.3 \%$, type II in $43.3 \%$ and type III in $1.4 \%$. Type II IM was further subdivided into subtype IIA (MUC1, 2, 5AC positive), IIB (MUC1, 2 positive) and IIC (MUC2, 5AC positive). MUC5AC and multifocal IM were significantly associated with progression to gastric dysplasia $(\mathrm{p}<0.05)$. Type III IM was associated with the highest rate of progression to dysplasia.

\begin{tabular}{|l|l|l|l|l|l|}
\hline First biopsy & \multicolumn{2}{|l|}{$\begin{array}{l}\text { Dysplasia/carcinoma } \\
\text { Yes No }\end{array}$} & OR & CI & p value \\
\hline Type I & 4 & 298 & Ref & Ref & Ref \\
\hline Type IIA & 8 & 87 & 6.85 & $2.01-23.29$ & 0.002 \\
\hline Type IIB & 1 & 30 & 2.48 & $0.26-22.92$ & 0.423 \\
\hline Type IIC & 9 & 100 & 6.70 & $2.02-22.24$ & 0.002 \\
\hline Type III & 2 & 6 & 24.83 & $3.78-162.73$ & 0.001 \\
\hline
\end{tabular}

Predictive power of combined MUC5AC expression and multifocal IM were higher than individual markers and clinicopathological features.

Conclusions: Incomplete IM (except type IIB) were significantly associated with the risk of progression to dysplasia/adenocarcinoma. Multiple biopsies should be evaluated in patients with IM to identify multifocal IM and MUC5AC expression could be helpful to assess the progression to gastric dysplasia or adenocarcinoma.

814 Loss of Switch/sucrose Nonfermenting Complex Protein Expression in Undifferentiated Gastrointestinal and Pancreatic Carcinomas

Basile Tessier-Cloutier, David Schaeffer, Julinor Bacani, Martin Koebel, Cheng-Han Lee. University of British Columbia, Vancouver, BC, Canada; University of Alberta, Edmonton, AB, Canada; University of Calgary, Calgary, AB, Canada; British Columbia Cancer Agency, Vancouver, BC, Canada.

Background: Undifferentiated carcinoma refers to an epithelial malignancy that lack morphologic evidence of differentiation. Recent studies have implicated the loss of constitutively expressed switch/sucrose nonfermenting (SWI/SNF) complex subunits in undifferentiated carcinomas of the gastrointestinal tract and the gynecologic tract, particularly in a mismatch repair (MMR) protein-deficient context. We examine here the expression of SWI/SNF and MMR proteins in a series of undifferentiated carcinomas from the gastrointestinal tract and the pancreas.

Design: We identified 22 cases of undifferentiated carcinoma found on resection specimens in pathology archives. Upon review, 7 were reclassified as poorly differentiated carcinoma based on the presence of focal glandular differentiation and 15 cases were confirmed to be undifferentiated carcinomas. Immunohistochemical analysis of SMARCA4, SMARCB1, ARID1A, ARID1B, MSH2, MSH6, MLH1 and PMS2 was performed on all cases.

Results: Among the 15 review-confirmed undifferentiated carcinomas ( 7 colonic, 4 gastric, 3 pancreatic and 1 duodenal), 9 were pure undifferentiated carcinomas and 3 contained differentiated component with gland formation. All except one tumor were associated with advanced disease stage and 9 of 15 tumors were MMR proteindeficient. 2 tumors showed loss of BRG1 (1 with concurrent ARID1A loss) and 2 showed loss of INI1 (1 with concurrent ARID1A loss). 1 tumor showed concurrent ARID1A and ARID1B loss where ARID1A expression was absent in both differentiated and undifferentiated component while ARID1B expression was absent only in the undifferentiated component. 4 additional tumors showed loss of ARID1A only. BRG1, INI1 or ARID1B-deficient undifferentiated carcinoma consistently exhibited sheet-like growth pattern, with cellular discohesion and rhabdoid morphology. In comparison, none of the 7 poorly differentiated carcinomas that were originally diagnosed as undifferentiated carcinomas showed loss of BRG1, INI1 or ARID1B.
Conclusions: Undifferentiated gastrointestinal/pancreatic carcinomas show frequent loss of expression of SWI/SNF complex proteins. The loss of these key components of SWI/SNF complex may contribute to the arrest of cellular differentiation, resulting in the undifferentiated histology and aggressive clinical behavior.

815

Anal Canal Adenocarcinoma with Associated Perianal Paget's Disease: An Underrecognized Entity with Institutional Experience Sarah C Thomas, Carolina Dominguez Marquez, Cory Porteus, Kun Jiang. Moffitt Cancer Center, Tampa, FL; University of South Florida, Tampa, FL.

Background: Anal canal adenocarcinoma(ACA) is a unique extraluminal cancer arising from anal mucosal glands that is particularly underrecognized both clinically and histopathologically. ACA is frequently misclassified as a colorectal or skin adnexal primary, due to its unusual location and ambiguous histopathology, especially when associated with extramammary perianal Paget's disease (EMPPD). Reviewing our institutional archive indicates that ACA often presents a diagnostic dilemma due to its insidious nature and close association with EMPPD

Design: We have encountered ACA cases that were initially misdiagnosed and furthermore, improperly managed as primary mucosal lesions. Twenty wide local excisions (WLE) of EMPPD, and one perianal excisional biopsy, coupled with endoscopic, radiologic and clinicopathological findings were retrieved and reviewed. Immunohistochemistry of cytokeratin 7 and 20, GCDFP, GATA3, CDX2, CK5/6, P40 and P63 were performed. Next generation sequencing and analysis of mismatch repair proteins were carried out when an invasive process was identified.

Results: All 20 EMPPD cases were clinically believed to be intramucosal processes, due to unremarkable colonoscopic and radiologic studies. Upon review however, 2 of them showed invasive extraluminal ACA, composed of mucin, glands and signet ring cells, with a unique immunophenotype of CK7+/CK20+/CDX2+/GCDFP-/GATA3-/ P40-/P63-, identical to the EMPPD component. The remaining 18 EMPPD cases were confirmed to be mucosal-restricted processes only, with a distinct CK7+/CK20-/CDX2-/ GCDFP+/GATA3+ immunoprofile. Follow-up colonoscopies confirmed the absence of a gastrointestinal lesion in the 20 cases. The perianal excisional biopsy showed ACA only, with no EMPPD, however it revealed inguinal lymph node metastasis. All three ACA patients underwent neoadjuvant therapies followed by a margin-free radical resection. Follow-ups at 20 months showed no recurrent disease.

Conclusions: A diagnosis of ACA should be entertained when facing any EMPPD, considering its insidious onset, poorly understood clinical course, and challenging management. Missing a diagnosis of ACA in an EMPPD patient could adversely impact the clinical course. Awareness of its immunohistological profile and deceptive EMPPD-like presentation may shed light on this infrequent, and yet significant entity.

816 Expect the Unexpected: Gastrointestinal Microsatellite Instable Adenocarcinoma without Characteristic Histopathological Features

Sarah C Thomas, Sherma Zibadi, Kun Jiang. Unviersity of South Florida, Tampa, FL; Moffitt Cancer Center, Tampa, FL.

Background: Microsatellite instability (MSI) status in gastrointestinal adenocarcinoma impacts the clinical outcome of patients, as an important prognostic and predictive factor for response to conventional Fluorouracil-based neoadjuvant treatment regimens. It has been widely accepted that MSI tumors display typical histomorphological characteristics such as rich mucin deposition, lymphoplasmacytic infiltration and medullary or signet ring morphology. However, our institutional review has identified frequent MSI tumors without these known microscopic features.

Design: 307 colorectal and 53 small intestinal adenocarcinoma resection cases from 2012 to 2015 were reviewed. The electronic chart, histopathology, immunohistochemistry and next generation sequencing (NGS) was analyzed and compared to published data findings associated with known MSI tumors.

Results: 68 of $307(22.2 \%)$ colorectal and 8 of 53 small intestinal tumors displayed MSI, identified by immunohistochemistry. Histologically, 23 of the $68(34 \%)$ colorectal MSI tumors showed no typical traits of MSI morphology; the average age of these 23 cases was 64.7 years with a male to female ratio of 0.8 . Notably, 9 of these 23 $(39 \%)$ involved non-right colon sites, mimicking microsatellite-stable (MSS) tumors. Immunohistochemistry revealed a loss of MLH1 and PMS2 in 14 (60.9\%), MSH2 and MSH6 in $5(21.7 \%)$, PMS2 in $3(13.0 \%)$, and MLH1, PMS2 and MSH6 in 1 case (4.3\%) among the 23 non-right sided MSI tumors.

In the 8 small intestinal MSI tumors, the average age was 57.4 years with a male to female ratio of 1.7 , with 5 of $8(62.5 \%)$ involving the duodenum, and 6 of $8(75 \%)$ being sporadic. Histologically, these 6 sporadic tumors appeared moderately to poorly differentiated and indistinguishable from their MSS counterparts. These cases demonstrated a loss of MLH1 and PMS2 in $4(50.0 \%)$, MSH2 and MSH6 in $3(37.5 \%)$, and PMS2 in $1(12.5 \%)$.

Conclusions: Our investigation illustrates that more than one third of colorectal, and a majority of small intestinal MSI tumors do not display dependable histopathological features. In fact, MSI tumors may share a wide spectrum of both clinical and histomorphological features with MSS tumors. These findings encourage a more comprehensive approach to identifying MSI. Even in the absence of characteristic histological features, MSI testing should be uniformly performed to best guide effective and targeted therapeutic strategies. 
817 Aneuploidy Detected by DNA Flow Cytometry Using ParaffinEmbedded Tissue Can Serve as Both Diagnostic Marker of Dysplasia and Predictive Marker of Neoplastic Progression in Inflammatory Bowel Disease

Jia-Huei Tsai, Peter S Rabinovitch, Thomas Small, Danning Huang, Aras N Mattis, Sanjay Kakar, Won-Tak Choi. UCSF Medical Center, San Francisco, CA; University of Washington, Seattle, WA; SUNY Upstate Medical University, Syracuse, NY.

Background: Distinction between regeneration and dysplasia, and between high grade dysplasia (HGD) and low grade dysplasia (LGD) can be challenging in inflammatory bowel disease (IBD), and reliable ancillary tests are not available. Flow cytometric analysis of DNA content (aneuploidy) has shown promise in stratifying IBD patients into low or high risk for colorectal cancer (CRC). These studies have typically used fresh tissue, which is not practical for integrating into the work flow, and does not allow direct correlation of flow cytometry results with morphologic findings. This study aims to evaluate if aneuploidy detected by flow cytometry using formalin-fixed paraffin-embedded (FFPE) tissue can aid in the diagnosis and risk stratification of dysplasia in IBD.

Design: DNA flow cytometry was performed using FFPE tissue from 18 flat HGD, 22 flat LGD, and 12 IBD without dysplasia. Three to four 60 micron thick sections were cut from each block, and area of interest was dissected for analysis.

Results: Aneuploidy was detected in $15(83 \%)$ flat HGD, $9(41 \%)$ flat LGD, and IBD $(8 \%)$ case without dysplasia. Forty-four percent of LGD cases with aneuploidy progressed to HGD and/or CRC at 1 year $(p=0.007)$, with $44 \%$ and $72 \%$ progression at $4(\mathrm{p}=0.115)$ and 12 years $(\mathrm{p}=0.072)$, respectively. By contrast, the 1-, 4-, and 12year progression rates of HGD and/or CRC for LGD cases without aneuploidy were $0 \%, 13 \%$, and $25 \%$, respectively. The hazard ratio for progression to HGD and/or CRC for patients with DNA aneuploidy detected at baseline LGD was 3.83 ( $p=0.093 ; 95 \%$ confidence interval, 0.795-27.3)

Conclusions: DNA flow cytometry using FFPE tissue can be helpful in establishing the diagnosis and risk stratification of dysplasia in IBD. The majority of HGD cases demonstrate aneuploidy, which can be helpful to confirm or establish the diagnosis of HGD in challenging cases. The finding of DNA aneuploidy at baseline LGD is predictive of progression to $\mathrm{HGD}$ and/or CRC.

\begin{tabular}{|c|c|c|c|}
\hline & No dysplasia $(\mathrm{n}=12)$ & Flat LGD $(\mathrm{n}=22)$ & Flat HGD $(\mathrm{n}=18)$ \\
\hline Aneuploidy & $1(8 \%)$ & $9(41 \%)$ & $15(83 \%)$ \\
\hline Progression to & & $44 \%$ (with aneuploidy) & \\
HGD/CRC at 1 year & & $0 \%$ (without aneuploidy) & \\
\hline
\end{tabular}

818 Normative Diagnostic Concordance for Low-Grade Appendiceal Mucinous Neoplasms and Related Primary Appendiceal Lesions

Mark A Valasek, Irene Thung, Esha Gollapalle, Alexey A Hodkoff, Vera Vavinskaya, Joel M Baumgartner, Andrew M Lowy. University of California San Diego, San Diego, CA; University of California San Diego, La Jolla, CA.

Background: Low-grade appendiceal mucinous neoplasm (LAMN) is known to cause the majority of pseudomyxoma peritonei (i.e. mucinous ascites); however, recognition and proper classification of these neoplasms can be difficult despite established diagnostic criteria.

Design: To determine the pathological diagnostic concordance for LAMN and related lesions during patient referral to an academic medical center specialized in treating patients with pseudomyxoma peritonei. The anatomic pathology laboratory information system was searched to identify cases over a two-year period containing appendix specimens with mucinous neoplasia evaluated by an outside pathology group and by in-house slide review at a single large academic medical center during patient referral. Results: 161 cases containing appendix specimens were identified over this period. Forty-six of 161 cases $(28.6 \%)$ contained appendiceal primary neoplasia or lesions. Twenty- nine of those $(63.0 \%)$ were diagnosed as LAMN, $13(28.3 \%)$ as adenocarcinoma, and $4(8.7 \%)$ as ruptured simple mucocele by the reference pathologist. The overall diagnostic concordance rate for these major classifications was $71.7 \%$ ( 33 of 46) with an unweighted observed kappa value of 0.48 ( $95 \%$ CI, $0.27-0.69)$, consistent with moderate interobserver agreement. All of the observed discordance could be attributed to overinterpretation by the initial pathologist. Moreover, $58.6 \%$ of LAMN had diagnostic reporting deficiencies.

Conclusions: Appendiceal mucinous lesions remain a difficult area for appropriate pathological classification and were miscategorized and overinterpreted in $28.3 \%$ of the cases in this study. The findings highlight the critical need for recognition of LAMN and perhaps clarification of diagnostic criteria regarding these tumors. Recently published consensus guidelines may help facilitate improvement of diagnostic concordance. We recommend expert secondary pathological review at large academic referral centers for these cases. Further studies are needed to determine the extent of this phenomenon and its potential clinical impact.
819 Small Bowel Carcinomas Associated with Celiac Disease Are Characterized by the Concomitant Presence of Nuclear $\beta$-Catenin Accumulation and Microsatellite Instability

Alessandro Vanoli, Michele Martino, Daniela Furlan, Federica Grillo, Gabriella Nesi, Claudia Mescoli, Daniele Fornino, Ombretta Luinetti, Fausto Sessa, Antonio Di Sabatino, Gino R Corazza, Enrico Solcia. University of Pavia, Fondazione IRCCS Policlinico San Matteo, Pavia, Italy; University of Insubria, Varese, Italy; San Martino/ IST University Hospital, Genova, Italy; University of Florence, Florence, Italy; University of Padua, Padua, Italy.

Background: Small bowel carcinomas (SBC) are relatively rare neoplasms, the majority of which arise sporadically; an increased risk for $\mathrm{SBC}$ has been found in both celiac disease $(\mathrm{CD})$ and Crohn's disease (CrD). SBC associated with CD (CD-SBC) have been reported to harbor a higher incidence of microsatellite instability (MSI) in comparison with sporadic SBC (spo-SBC i.e. primary non-familial SBC not associated with either $\mathrm{CD}$ or $\mathrm{CrD}$ ). On the contrary, MSI has been rarely observed in SBC associated with $\mathrm{CrD}$ (CrD-SBC). $\beta$-catenin nuclear expression has been reported in less than half of spo-SBC; however, data regarding $\beta$-catenin in CD-SBC and CrD-SBC are very limited. Design: We aimed to explore the immunohistochemical expression of $\beta$-catenin and mismatch repair proteins in 76 primary, non-ampullary, non-familial SBC, including 26 CD-SBC, $25 \mathrm{CrD}-\mathrm{SBC}$ and $25 \mathrm{spo}-\mathrm{SBC}$. MSI status was also assessed using a pentaplex panel of monomorphic mononucleotide repeats in all cases. MLHI methylation status was examined by pyrosequencing in all the cases exhibiting loss of MLH1 expression. Results: MSI was found in 25 out of $76 \mathrm{SBC}(33 \%)$ and no discordance between immunohistochemistry and molecular analysis assays was observed. A significantly higher frequency of MSI was observed in CD-SBC compared with either CrD-SBC or spo-SBC ( $<<0.001$ for both). All MSI tumors showed a loss of both MLH1 and PMS2 immunohistochemical expression while retaining MSH2 and MSH6 expression and all but one harbored $M L H 1$ methylation. Nuclear expression of $\beta$-catenin was more frequently observed in CD-SBC $(92 \%)$ in comparison with either CrD-SBC $(22 \%$, $\mathrm{p}<0.001)$ or spo-SBC $(36 \%, \mathrm{p}<0.001)$. Most CD-SBC $(65 \%)$ showed the presence of both nuclear $\beta$-catenin and MSI in contrast to only one CrD-SBC $(\mathrm{p}<0.001)$ and two spo-SBC $(\mathrm{p}<0.001)$

Conclusions: $\mathrm{CD}-\mathrm{SBC}$ are characterized by nuclear localization of $\beta$-catenin, an indirect evidence of deregulated Wnt signaling pathway. At variance with other SBC and also colorectal cancers, CD-SBC often show the concomitant occurrence of nuclear $\beta$-catenin expression and MSI, suggesting a distinctive molecular pathogenesis.

820 Tumor Buds and Micropapillae in Colorectal Carcinoma Demonstrate Similarities in Biological Behavior and May Represent Two Ends of the Same Spectrum

Monika Vyas, Romulo Celli, Xuchen Zhang. Yale School of Medicine, New Haven, CT. Background: Micropapillary colorectal carcinoma (MPC) has been reported as an aggressive variant of carcinoma associated with frequent lymphovascular invasion and poor outcome. The micropapillary components $(\mathrm{MC})$ are clusters of closely adherent neoplastic cells, which are located in distinct empty spaces. While a single cell or clusters of up to 5 tumor cells were considered as a tumor bud, high tumor budding is also a hallmark of unfavorable tumor biology correlating with advanced tumor stage, lymphovascular invasion and metastasis. Increased expression of glucose transporter-1 (GLUT-1) has been correlated with tumor cell survival, tumor progression and poor survival in several cancer types. The goal of this study was to compare the biologic behavior of TB and MC in terms of proliferation index and metabolic profile by studying GLUT-1 expression. We speculate that tumor buds (TB) and MC are a part of the same spectrum.

Design: 10 cases of colorectal carcinoma (CRC) with $\mathrm{MC}$ and 15 cases of $\mathrm{CRC}$ with high TB but without MC were included in this study. High tumor budding was based on $>=10$ buds in a $200 \mathrm{X}$ objective field criterion. Immunostains for Ki- 67 and GLUT- 1 were performed. Expression of GLUT-1 was studied in the MC, TB and adjacent tumor cells of glandular component. For analysis of proliferation index (Ki-67), 3 high power fields (400X) of MC/TB component and adjacent tumor cells of glandular component were counted and calculated as percentage of Ki-67 positive cells out of total cells.

Results: All the 10 CRC with MC cases also showed high grade TB. GLUT-1 was more strongly expressed in both $\mathrm{MC}$ and $\mathrm{TB}$ cells as compared to adjacent tumor cells of glandular component in 19/20 cases. The proliferation index (Ki-67) of MC was significantly lower compared to adjacent tumor cells of glandular component $(13.63 \% \pm 6.14$ vs. $66.49 \% \pm 4.35, \mathrm{P}<0.001)$. Similarly, TB cells also showed significantly lower proliferation as compared to adjacent tumor cells of glandular component $(6.6 \% \pm 2.3$ vs. $76.3 \% \pm 9.6, \mathrm{p}<0.001)$.

Conclusions: Our study demonstrates that high grade TB is often seen in CRC with MC. Both of the MC and TB component show increased GLUT-1 expression and decreased proliferation index. The reprogramming of glycogen metabolism by increased expression of GLUT-1 may help in providing nutrition to the MC and TB cells in a low proliferation state and aid aggressive biological behavior. This similarity in tumor biology, in addition to histology, may indicate that TB and MP are a part of the same spectrum.

821 Isolated Intestinal Metaplasia in Gastric Antrum Kai Wang, Lei Zhao. University of Alabama at Birmingham, Birmingham, AL. Background: Isolated intestinal metaplasia (or intestinal metaplasia without other pathologic change) in stomach antrum is occasionally encountered in daily practice. How to manage these patients clinically is unclear due to lack of understanding of clinical significance of this finding. In this retrospective study, we analyzed the incidence and collected the clinical followup information of isolated intestinal metaplasia. Design: We retrieved all the patients who had upper GI endoscopy with pathology diagnosis of intestinal metaplasia in stomach antrum between 2004 and 2014. Clinical follow-up for these patients were collected through electronic medical record. 
Results: A total of 119 patients with antral intestinal metaplasia were identified, 54 females and 65 males. The average age is 62 (range: $24-86$ ). The median documented clinical (endoscopic exam without biopsy) or pathological (with biopsy) follow-up interval is 4 years (range: 1 month to 12 years). Reviewing the index biopsies revealed 27 cases $(23 \%)$ with isolated intestinal metaplasia and 92 cases $(77 \%)$ with background gastritis or significant chronic inflammation in addition to intestinal metaplasia. Among the patients who had background gastritis, 17 patients had $\mathrm{H}$ pylori (by routine or special stain) and 3 had low-grade dysplasia.

While all patients received clinical follow-up, 18 patients (5 without gastritis and 13 with gastritis) had undergone repeat biopsies. Nine patients $(50 \%)$ showed persistent intestinal metaplasia in follow-up biopsy. Eight of them $(89 \%)$ had background gastritis and one had isolated intestinal metaplasia in the index biopsies. One 71-year-old female patient with gastritis and low grade dysplasia in the index biopsy developed gastric adenocarcinoma. No other patients developed carcinoma in the follow-up studies. Conclusions: Antral intestinal metaplasia encompasses heterogenous groups of conditions. And the risk of malignant transformation is likely to be very different among the groups. This study suggests that isolated antral intestinal metaplasia represents a significant percentage of gastric intestinal metaplasia in daily practice and many probably represent an incidental finding due to sampling of transitional type epithelium adjacent to pylorus channel. Therefore it is worth further delineating different types of intestinal metaplasia in future large-scale clinical studies.

822 Programmed Death-Ligand 1 Expression in Gastric Cancer: Correlation with Mismatch Repair Deficiency and HER2-Negative Status Lei Wang, Shengnan Zhao, Shujuan Ni, Cong Tan, Dan Huang, Weiqi Sheng. Fudan University Shanghai Cancer Center, Shanghai, China.

Background: Gastric cancer (GC) is one of the most common malignancies, especially in Asia. Trastuzumab, one kind of targeted therapies, can only be benefited by human epidermal growth factor receptor 2 (HER2) positive patients. The immune regulatory programmed death-1 (PD-1)/ programmed death-ligand 1 (PD-L1) axis, which protects the host from overactive T-cells, has been used as an immune checkpoint target for immunotherapy in various malignancies. In colorectal cancer, it has been demonstrated that patients with mismatch repair (MMR) deficiency are good responders to anti-PD-1/ PD-L1 immunotherapy. However, the indication of PD-L1 expression in GC remains controversial. The relationship between PD-L1 expression and the status of MMR proteins or HER2 expression needs to be understood profoundly.

Design: We retrospectively analyzed 571 consecutive cases of GC in our hospital from 2010 to 2012. PD-L1 and MMR protein were detected by immunohistochemistry (IHC). Positive PD-L1 expression was defined as at least $5 \%$ or more membranous and/ or cytoplasmic staining in either tumor cells or tumor immune cells. HER2 status was assessed by IHC, and followed by fluorescence in situ hybridization in IHC $2+$ cases. Results: PD-L1 was identified in 17.0\% (97/571) of GC. Deficient MMR (dMMR) could be detected in 48 patients $(8.4 \%)$ in our cohort. GC with dMMR showed higher rates of PD-L1 expression compared with MMR proficient carcinoma (31.3\%VS15.7\%, $P=0.006)$. PD-L1 was positive in 15 of 33 cases $(45.5 \%)$ without MLH1 and PMS2 expression $(P<0.001)$, while only in 3 cases of 18 MSH6 negative cases $(16.7 \%)$. For those 3 MSH2 negative cases, PD-L1 expression could not be detected. HER2 was positive in $61(10.7 \%)$ cases, among which only three were positive for PD-L1 (4.9\%). However, in HER2-negative group, 18.4\% (94/510) of tumors were positive for PD-L1 $(P=0.004)$. Univariate analysis showed that PD-L1 was a positive prognostic factor for disease free survival (DFS) $(P=0.002)$ and overall survival (OS) $(P=0.004)$. Multivariate analysis showed that PD-L1 expression was an independent predictor of DFS $(P=0.013)$ and OS $(P=0.004)$.

Conclusions: This study is the first evaluation of PD-L1 expression in a large Asian cohort of GC. PD-L1 is an independent positive prognostic factor and correlated with dMMR and HER2-negative status. Our finding indicated that MMR and HER-2 status may be potential biomarkers for anti-PD-L1 therapy.

\section{Perianal Paget's Disease: Experience of a Single Institution}

Yu-Chen Wang, Wen-Yih Liang, Anna Fen-Yau Li. Taipei Veterans General Hospital, Taipei, Taiwan; National Yang-Ming University, Taipei, Taiwan.

Background: The perianal Paget's diseases (PPDs) are the second most common location of extramammary Paget's disease and can be associated with or without colorectal cancer. This study was designed to evaluate the immunophenotype and prognosis of PPDs.

Design: 23 patients with PPDs through tissue proved at the Taipei Veterans General Hospital during 2000 and 2015 were identified by a retrospective search of pathology records. Patient demographics, treatments, histopathological features, and long-term outcomes were documented. The expression of the CK7, CK20 and CDX2 of the PPDs by immunohistochemical (IHC) method is performed, and all slides were reviewed by two pathologists. The clinical and pathological stage of the CRCs and the outcomes were evaluated.

Results: Among the 23 PPD patients, 12 have concurrent CRC at the time of PPD diagnosis ( 8 anal canal, 4 rectal; 7 stage I or II, 4 stage III or IV, 1 unknown due to refusing operation and further treatment). All cases are separated in four groups according to the concurrent $\mathrm{CRCs}$ and expression of CDX2 in the PPDs: $\mathrm{CRC}+/ \mathrm{CDX} 2+$ (11 cases, $47.9 \%$ ), $\mathrm{CRC}+/ \mathrm{CDX} 2-(1$ case, $4.3 \%)$, CRC-/CDX2+ ( 7 cases, $30.4 \%$ ), and CRC-/CDX2- (4 cases, 17.4\%). The recurrent rates (including PPD local recurrence or CRC distant metastasis during follow-up) of patients with PPD and concurrent CRC are $42.9 \%(3 / 7)$ for stage I/II and $50.0 \%(2 / 4)$ for stage III/IV.
Demographics and outcomes in the eleven PPD patients with CRC

\begin{tabular}{|l|l|l|l|l|l|l|}
\hline Case & Age & Sex & CRC location & $\begin{array}{l}\text { CRC TNM } \\
\text { stage at } \\
\text { initial } \\
\text { diagnosis }\end{array}$ & $\begin{array}{l}\text { Recurrent } \\
\text { interval of PPD }\end{array}$ & $\begin{array}{l}\text { Interval of } \\
\text { CRC distant } \\
\text { metastasis } \\
\text { during } \\
\text { follow-up }\end{array}$ \\
\hline 1 & 72 & Female & Rectum & T3N0Mx & N/A & N/A \\
\hline 2 & 71 & Female & Rectum & T3N0Mx & 10 years & N/A \\
\hline 3 & 73 & Male & Anorectum & T2N0Mx & N/A & N/A \\
\hline 4 & 77 & Female & Anorectum & T1N0Mx & N/A & N/A \\
\hline 5 & 77 & Male & Anorectum & T3N2aMx & N/A & 21 months \\
\hline 6 & 77 & Male & Anorectum & T1N0Mx & N/A & 24 months \\
\hline 7 & 73 & Male & Rectum & T1N1aMx & N/A & N/A \\
\hline 8 & 50 & Female & Anorectum & T2N0Mx & N/A & 20 months \\
\hline 9 & 88 & Male & Anorectum & cT2N0Mx & N/A & N/A \\
\hline 10 & 81 & Male & Anorectum & T3N1bMx & N/A & 13 months \\
\hline 11 & 79 & Male & Anorectum & T3N2bMx & N/A & N/A \\
\hline
\end{tabular}

Conclusions: Our results reveal that CDX2 is a very useful antibody in differential diagnosis of PPD. Nodal negative CRCs with Paget's disease had a much higher recurrent rate than same stage CRCs without Paget's disease. Upstaging of these cases and more aggressive intervention such as adjuvant chemotherapy would be considered for these patients.

\section{Taiwan Hospital-Based Detection of Microsatellite Instabilities} and BRAF Mutation in Colorectal Cancer by Immunohistochemical Method Yu-Chen Wang, Wen-Yih Liang, Anna Fen-Yau Li. Taipei Veterans General Hospital, Taipei, Taiwan; National Yang-Ming University, Taipei, Taiwan.

Background: Microsatellite status plays an important role in colorectal carcinomas (CRCs), which would affect the chemotherapy administration, especially 5 -fluorouracilbased adjuvant chemotherapy, in advanced stage cases. The microsatellite status evaluation could also be a screening tool for Lynch syndrome. DNA mismatch-repair (MMR) proteins immunohistochemistry (IHC) facilitates universal screening of CRCs for microsatellite status: microsatellite stability (MSS) or microsatellite instability (MSI). The MMR proteins defect in CRCs of Taiwanese was investigated by the immunohistochemical method and this is a retrospective report of the experience in a medical institution in Taiwan.

Design: 439 patients with CRCs receiving colectomy at the Taipei Veterans General Hospital in 2012 were identified by a retrospective search of pathology records. All slides were reviewed by two pathologists, and MMR status and BRAF V600E mutation detection by IHC are performed. Associations between MMR defect and patient's stage and age were also evaluated.

Results: Among the 439 patients, 14 patients had been diagnosed with intramucosal adenocarcinoma in the polypectomy or mucosal resection specimen and there was no residual tumor in the colectomy specimen. These patients were excluded and the residual 425 patients are involved. BRAF V600E polymerase chain reaction (PCR) and MMR IHC demonstrated the following phenotypes: BRAF-/MSS (391 cases, 92.0\%), BRAF+/MSS (12, 2.8\%), BRAF+/MSI (9, 2.1\%), and BRAF-/MSI (13 cases, 3.1\%). Among the 13 BRAF-/MSI cases, 8 (61.5\%) show loss of MLH1 and PMS2 expression, $4(30.8 \%)$ show loss of MSH2 and MSH6 expression, and $1(7.7 \%)$ shows loss of MLH1, PMS2, MSH2 and MSH6 expression. Only 1 patient with loss of MLH1 and PMS2 expression has certain family history of CRC. Among advanced stage cases (stage III or IV), comparing the association between the microsatellite status (MSS or MSI) and the age ( $>70$ years or $\leq 70$ years), there is no statistically significant difference. $(p=1.0)$ Conclusions: Our results reveal that the frequency of MSI of CRCs in Taiwanese is approximately $5.2 \%$, while the frequency of BRAF-/MSI cases is about $3.1 \%$. BRAF IHC is very useful in the screening of Lynch syndrome and the combination of IHC for MMR and BRAF should be considered in all stage and age groups for Lynch screening, treatment guidance and prognosis prediction.

825 SMAD4 Immunohistochemical Expression Loss in Esophageal Adenocarcinoma

Kevin Waters, Ogechukwu Pearl Eze, Maryam Pezhouh, Yaman Tarabishy, Lysandra Voltaggio, Elizabeth A Montgomery. Johns Hopkins Hospital, Baltimore, MD.

Background: SMAD4 (DPC4), a tumor suppressor gene, is often altered in pancreatic adenocarcinoma. In the pancreas, loss of nuclear expression by immunolabeling mirrors mutational status, and is used to suggest pancreatic origin in metastasis of unknown origin. On whole exome and genome sequencing of 149 esophageal adenocarcinomas (EAC), $8 \%$ carried SMAD4 mutations and $34 \%$ had copy number loss. Another study found SMAD4 loss by IHC in $10 \%$ of EACs; these cases were associated with shorter time to recurrence and overall survival than intact tumors. We further characterize the relationship between EAC and loss of SMAD4 expression.

Design: TMAs from 88 EAC resections between 1984 and 2004 were immunostained with SMAD4. Cases were considered to have SMAD4 loss if there was absent nuclear staining in all tumor cells. Demographic and pathology data were analyzed and compared using T-tests for comparison of means, Mann-Whitney U tests for non-parametric data, and Fischer exact tests for $2 \times 2$ contingency tables.

Results: The mean age at resection was $66,90 \%$ were male, and $94 \%$ were white. Loss of staining was detected in $28 \%(25 / 88 ; 95 \%$ confidence interval $19-38 \%)$. The mean number of cores examined was slightly higher in cases with loss (2.5) than those without (2.3) as was the proportion of cases with loss $(30 \% ; 10 / 33)$ when limited to those with 3 or more cores. In cases with SMAD4 loss and cores with high-grade dysplasia (HGD), 
SMAD4 staining was intact in the HGD. No statistically significant differences were found between the average age, grade, tumor size, or extent of invasion (pT) between tumors with and without SMAD4 loss. Cases with loss had higher median nodal stage $(\mathrm{p}=0.048$ ) with $68 \%$ having nodal metastases (vs. $47 \%$ ). While not statistically significant, median survival was lower in patients with SMAD4 loss (12 vs. 20 months, $\mathrm{p}=0.41)$, and they were less likely to survive $\geq 5$ years $(10 \%$ vs. $27 \%, \mathrm{p}=0.13)$ and $\geq 10$ years $(0 \%$ vs. $17 \%, \mathrm{p}=0.10)$.

Conclusions: A subset of EACs lacks SMAD4 immunolabeling indicating that, in addition to pancreas, an esophageal primary needs to be excluded in metastases of unknown primary with SMAD4 loss. The increased proportion of loss in our cases compared to the prior report may reflect a different population and that our cases were collected at an earlier timepoint with different protocols for resection and neoadjuvant therapy. We did not see SMAD4 loss in precursor lesions supporting the hypothesis that SMAD4 mutation is a late event in EAC tumorigenesis. While not statistically significant, our data directionally support prior data showing that SMAD4 mutations indicate a worse prognosis.

826 Genomic Landscape in Appendiceal Goblet Cell Carcinoid: Distinct Profile Compared to Neuroendocrine Tumor and Colorectal Adencoarcinoma

Kwun Wah Wen, James Grenert, Nancy Joseph, Nafis Shafizadeh, Mojgan Hosseini, Anne Huang, Sanjay Kakar. UCSF, San Francisco, CA; Kaiser, Woodland Hills, CA; UCSD, San Diego, CA; Vista Pathology, Medford, OR.

Background: Goblet cell carcinoid (GCC) is a rare appendiceal tumor and can have an additional adenocarcinoma (AC) component. Both GCC and GCC-AC are staged and treated like AC. The word "carcinoid" in GCC frequently leads to confusion with appendiceal neuroendocrine tumor (NET) that typically behaves as a low-grade tumor. This study examines the genomic landscapes of appendiceal NET, GCC and GCC-AC. Design: DNA was extracted from formalin-fixed paraffin embedded tissue from 10 tumors (3 NET, 2 GCC, 4 GCC-AC, 1 pure AC) with matched normal tissue. Capture-based next generation sequencing (NGS) targeting the coding regions of 479 cancer genes and select introns was performed. Somatic single nucleotide variants (SNV), insertions/ deletions (indels), copy number alterations (CNA), and selected rearrangements were evaluated.

Results: Coding mutations were not seen in any NET, but were present in all GCC (average 2.5) and GCC-AC cases (average 5.3). Mutations in chromatin remodeling genes were seen in all 4 GCC-AC cases (ARID1A: 2 cases; KMT2A: 1 case; KMT2B: 1 case; KMT2D: 1 case; KDM6A: 1 case). $S O X 9$ frameshift mutations (transcription factor involved in development of intestinal epithelium) were present in 2 GCC-AC cases ( 2 separate SOXY frameshift mutations in 1 case). 1 mutation in RHOA (Ras homolog gene family, member A; a small GTPase protein of Rho family) was seen in 1 GCC-AC case and 1 mutation in TP53 in another. A single pure AC case showed mutations in $A P C, K R A S$, and TP53. Mutations in APC, KRAS, PIK3CA, SMAD4, or pathogenic germline alterations were not identified in any other cases.

\begin{tabular}{|l|l|l|l|}
\hline & $\begin{array}{l}\text { Mutation } \\
\text { number }\end{array}$ & Pathogenic & Unknown significance \\
\hline $\begin{array}{l}\text { NET } \\
(\mathrm{n}=3)\end{array}$ & $0,0,0$ & N/A & N/A \\
\hline $\begin{array}{l}\text { GCC } \\
(\mathrm{n}=2)\end{array}$ & 2,3 & CDH1 & KMT2A, NCOR1, MAP3K2, ZFHX4 \\
\hline $\begin{array}{l}\text { GCC- } \\
\text { AC } \\
(\mathrm{n}=4)\end{array}$ & $3,4,6,8$ & $\begin{array}{l}\text { ARID1A (x2), KDM6A, } \\
\text { KMT2D, RHOA, SOX9 } \\
(\mathrm{x} 2), \text { TP53 }\end{array}$ & $\begin{array}{l}\text { KMT2A, KMT2B, IPMK, CIC, FGF23, } \\
\text { GNAS, CDK8, ARAF, NTRK1, INHBA, } \\
\text { SPEN, CLDN18 }\end{array}$ \\
\hline $\begin{array}{l}\text { Pure } \\
\text { AC } \\
(\mathrm{n}=1)\end{array}$ & 7 & APC (x2), KRAS, TP53 & AXIN1, ABL1, EDNRB \\
\hline
\end{tabular}

Conclusions: The mutational profile of GCC/GCC-AC is distinct from NET, and does not show mutations commonly observed in colorectal cancer including $A P C, K R A S$, PIK3CA, SMAD4, or TP53. Mutations in SOX9 and chromatin-modifier genes (ARIDIA, $C D H 1, K M T 2 D, K D M 6 A$ ) may play an important role in GCC and GCC-AC.

\section{Immune Infiltrates Confer Prolonged Survival in Colorectal} Cancer with Microsatellite Instability

David Williams, Marsali Newman, Elham Amini, David Nickless, Catherine Fang, Robert Jorissen, Oliver Sieber. Austin Health, Melbourne, Victoria, Australia; Sonic Healthcare, Perth, Western Australia, Australia; Australian Clinical Labs, Melbourne, Victoria, Australia; Olivia Newton-John Cancer Research Institute, Melbourne, Victoria, Australia; Walter and Elisa Hall Institute of Medical Research, Melbourne, Victoria, Australia.

Background: Microsatellite instability (MSI) occurs in about $15 \%$ of colorectal cancers (CRC), and confers a good prognosis. Several potentially prognostic pathological features are commonly associated with MSI CRC, including immune infiltrates, high grade, mucinous or signet ring differentiation, and BRAF mutation. We hypothesized that the presence of immune infiltrates is the main factor that confers prolonged survival in MSI CRC.

Design: A series of 938 colorectal cancers were reviewed, enriched for cases with confirmed MSI, or with pathological features common to MSI tumours (high grade, mucinous differentiation, BRAF mutation). Tumour infiltrating lymphocytes (TILs) were scored in each tumour by a semi-quantitative measure per 5 high power fields $(0.5 \mathrm{~mm}$ field diameter). Crohnoid infiltrates beyond the invasive front were scored as lymphoid aggregates per low power field ( $5 \mathrm{~mm}$ field diameter). Cases were also reviewed for conventional prognostic factors.
Results: MSI CRC was significantly associated with of origin in the proximal colon, female gender, elderly age, high grade, mucinous differentiation, BRAF mutation, TILs and Crohnoid infiltrates $(\mathrm{p}<0.05)$, consistent with previous reports. MSI CRC had a significantly lower incidence of stage 4 disease relative to MSS CRC $(p<0.05)$. In stage matched disease there was no difference in overall and relapse-free survival, but the presence of TILs or Crohnoid infiltrates were independently associated with prolonged survival in AJCC stage 3 MSI CRC.

Conclusions: Lymphocyte infiltrates are prognostic for a more favourable outcome in colorectal cancers with microsatellite instability. A subset of MSI CRCs lack immune infiltrates; the significance of this finding in the context of other potential poor prognostic factors will be discussed.

\section{Prognostic Impact of Grade and MSI Status in Mucinous} Colorectal Cancers

David Williams, Marsali Newman, Elham Amini, David Nickless, Catherine Fang, Robert Jorissen, Oliver Sieber. Austin Health, Melbourne, Victoria, Australia; Sonic Healthcare, Perth, Western Australia, Australia; Australian Clinical Labs, Melbourne, Victoria, Australia; Olivia Newton-John Cancer Research Institute, Melbourne, Victoria, Australia; Walter and Elisa Hall Institute of Medical Research, Melbourne, Victoria, Australia.

Background: Mucinous colorectal carcinoma (mucCRC) and mucin-producing colorectal carcinoma (mpCRC) are defined as tumours with $>50 \%$ and $<50 \%$ mucinous differentiation respectively. WHO guidelines recommend that conventional grading should not be applied to mucCRC, but that MSI status should be used to stratify risk in these tumours. We aimed to assess the validity of these guidelines in predicting the clinical behaviour of mucCRC and mpCRC.

Design: A series of 951 colorectal cancers was reviewed, enriched for cases with reported mucinous histology. Tumours were reviewed for presence and extent of mucinous differentiation, classified as mucCRC, mpCRC and non-mucinous CRC. WHO grading was applied to all tumours; in mucinous fields, strips of epithelium at the periphery of mucin lakes with retained nuclear polarity were regarded as low grade, whereas solid nests and sheets were regarded as high grade. Cases were also assessed for conventional prognostic factors, and for BRAF and MSI status. Relapse-free survival and overall survival were assessed in univariate and multivariate analyses.

Results: Both mucCRC and mpCRC were significantly associated with origin in the proximal colon, female gender, MSI, BRAF mutation, increased tumour infiltrating lymphocytes and less venous invasion $(\mathrm{p}<0.05)$. There was no significant difference in these features between mucCRC and $\mathrm{mpCRC}$, and there was no difference in patient outcomes. High grade conferred a poor prognosis in both mucinous and non-mucinous colorectal cancer. MSI was a good prognostic factor in mucCRC/mpCRC in univariate analysis, but not in multivariate analysis. Lymphocyte infiltrates were a better predictor of survival than MSI status.

Conclusions: MucCRC and mpCRC have similar clinicopathological and molecular features. The arbitrary distinction between mucCRC and mpCRC appears to have no biological or prognostic value. Assessment of grade was found to be prognostic despite current guidelines to the contrary. MSI status may play a role in determining prognosis, but this is likely due to the increased likelihood of lymphocyte infiltrates.

\section{Alterations in Lamina Propria and Muscularis Mucosa in Ulcerative Colitis Are Associated with Prior Medication and Degree of Histologic Inflammatory Activity}

Eric Willis, Rocio Lopez, Neha Agrawal, Florian Rieder, Ilyssa Gordon. Cleveland Clinic, Cleveland, $\mathrm{OH}$.

Background: Ulcerative colitis has classically been described as a mucosal disease, as compared to its counterpart Crohn's disease, which is transmural. Although it is known that ulcerative colitis affects the superficial submucosa, the range of histologic patterns that can be seen in chronic disease has not been described. Here we investigate changes to the lamina propria and muscularis mucosa that can be seen in ulcerative colitis. Design: Diagnostic H\&E stained sections taken approximately every $10 \mathrm{~cm}$ from 94 consecutive colectomy specimens for ulcerative colitis were reviewed and assessed for the presence of lamina propria fibrosis and for qualitative alterations in the muscularis mucosa, including uniform thickening, splitting, splaying, thickening not otherwise specified, loss associated with prominent lymphoid aggregates involving the mucosa and superficial submucosa, and loss without associated inflammation. Patterns were not exclusive and could be assigned if present focally. A predominant pattern was also assigned to each section. Geboes score was determined for each section to assess the degree of histologic inflammatory activity. Clinical characteristics were also collected. Results: All patients had a clinical and histologic diagnosis of ulcerative colitis. Cases were grouped according to predominant patterns. There was a significant difference between the groups $(\mathrm{p}=0.005)$ for prior use of 5 -aminosalicylic acid with the lowest use being those with a band pattern of lamina propria fibrosis and associated prominent lymphoid aggregates, and the highest use being those with some lamina propria fibrosis, a uniform thickening of the muscularis mucosa, and prominent lymphoid aggregates. Other prior medications with significant differences between the groups were use if any steroids $(\mathrm{p}=0.047)$, systemic steroids $(\mathrm{p}=0.047)$, and azathioprine $(\mathrm{p}=0.014)$. There were also significant differences between the groups $(\mathrm{p}<0.001)$ for Geboes score.

Conclusions: Ulcerative colitis resections reveal alterations of the muscularis mucosa and lamina propria which are related to prior medication use and to the degree of histologic inflammatory activity as assessed by the Geboes score. Pathologists should be aware of these histologic features of ulcerative colitis affecting the lamina propria and superficial submucosa so as not to misinterpret them as Crohn's disease. 
830 Neuroendocrine Proliferations in Inflammatory Bowel Disease Mary Wong, Brent Larson, Deepti Dhall. Cedars-Sinai Medical Center, Los Angeles, CA; Keck School of Medicine of the University of Southern California, Los Angeles, CA. Background: The pathogenic relationship between chronic inflammatory bowel disease (IBD) and neuroendocrine proliferations (NEPs) is ill defined, as these cases are rare. The findings are variable in multiple studies regarding the rate of NEPs in IBD, as well as the role of inflammation in the development of NEPs in IBD. This study investigates clinical and pathologic features to identify a discrete profile of features that could contribute to understanding the biology of NEPs in IBD.

Design: The institutional surgical pathology archives were searched to identify cases with both IBD and NEPs. Poorly differentiated neuroendocrine neoplasms were excluded. Glass slides, with associated immunohistochemical stains, were reviewed to investigate the pathologic features. Clinical, radiological, and laboratory findings were also reviewed via institutional electronic medical records.

Results: Twelve cases (median age 32, range 18-62; $\mathrm{M}: \mathrm{F}=7: 5$ ) were identified from a total of 3151 IBD cases from 1994-2016; including ulcerative colitis $(n=6)$, Crohn's disease $(\mathrm{n}=5)$, and indeterminate $(\mathrm{n}=1)$. In all 12 cases, NEPs were incidentally discovered. Three cases were classified as endocrine cell micronests $(\mathrm{ECMs})(\mathrm{each}<0.5$ $\mathrm{mm}$ ). The 9 remaining cases were well-differentiated neuroendocrine tumors (NETs) that were further classified into microcarcinoids (median size $2.0 \mathrm{~mm}$, range $1.0-3.0 \mathrm{~mm}$ ) and carcinoids (median size $8.0 \mathrm{~mm}$, range $5.0-11.0 \mathrm{~mm}$ ). Inflammation was identified in adjacent mucosa in $100 \%$ of ECMs and $66.7 \%$ of NETs. NEPs occurred in the rectum $(\mathrm{n}=6)$, appendix $(\mathrm{n}=3)$, rectosigmoid $(\mathrm{n}=2)$, and ileostomy $(\mathrm{n}=1)$. Four cases exhibited mucosal neuroendocrine cell hyperplasia adjacent to ECMs and NETs. There was no progression of ECMs or NETs at a median follow-up of 5 years (range 2-31 years). Conclusions: Compared to the rate of NETs in the SEER database, IBD patients in our institution were 2.2 times more likely to have NETs. A significant proportion of NEPs showed inflammation in the adjacent mucosa, suggesting that inflammation likely plays a role in its development. Furthermore, ECMs and well-differentiated NETs in IBD patients have an indolent clinical course.

831 Differentiating the Undifferentiated Revisited: Expression of Markers of Intestinal Differentiation, GPA33, CDX2, CDH17 and SATB2 in Medullary (Undifferentiated) Carcinoma of the Colon

Stephanie Wood, Anthony J Gill, Hai Wang, Kenneth Friedman, Kara A Lombardo, Murray B Resnick. Rhode Island Hospital, Providence, RI; OHSU, Portland, OR; Royal North Shore Hospital, St Leonards, Australia.

Background: Medullary carcinomas of the colon (MC) are a rare colorectal carcinoma (CRC) subset with a favorable prognosis despite its undifferentiated morphology. Medullary carcinomas may be difficult to separate from poorly differentiated (PD) CRCs and other undifferentiated tumors. We decided to test a large cohort of medullary carcinomas for multiple markers of intestinal differentiation, including a novel marker, GPA33. GPA33 is a cell surface antigen that is expressed in normal colonic epithelium. Design: Immunohistochemical (IHC) analysis of CDX2, CDH17, SATB2, and GPA33 was performed. TMAs were constructed from Rhode Island Hospital cases, including MC ( $n=7$ ), well differentiated MSI CRCs (WD MSI, $n=25$ ), well differentiated microsatellite stable CRCs (WD MSS, n=71), and PD CRCs ( $\mathrm{n}=56$ ). Additionally, samples of MC $(n=92)$ were microarrayed by the Royal North Shore Hospital. Tumors were scored positive if more than $10 \%$ of the tumor exhibited at least moderate staining. Statistical analysis was performed using the Chi-squared test.

Results: IHC expression of all markers was decreased in MCs when compared to nonMCs (Figure 1), including PD CRC. GPA33 stained more MCs than all other markers, although this difference was only statistically significant when compared to SATB2 expression $(\mathrm{p}<0.0001)$. There were $22 \mathrm{MCs}$ negative for CDX2, CDH17, and SATB2. Of these 22 triple-negative MCs, 7 (32\%) were positive for the newer IHC marker GPA33. Intriguingly SATB2 was also decreased in $40 \%$ of well differentiated MSI tumors.

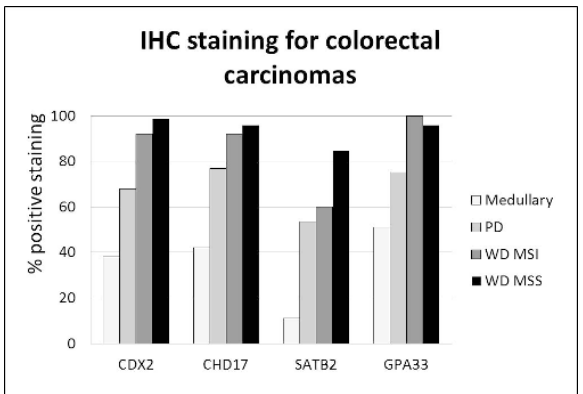

Conclusions: In this large cohort $(\mathrm{n}=99)$ of MCs we found decreased expression of intestinal markers, including the novel IHC marker, GPA33. Although recent studies suggest that $\mathrm{CDH} 17$ and SATB2 may be reliable markers for MC, our data is less supportive. MCs were more often positive for GPA33 than for the other markers of intestinal differentiation, staining $32 \%$ of MCs that were negative for all other markers. GPA33 may be a helpful marker to determine intestinal differentiation in poorly differentiated and medullary colorectal carcinomas.
832 Cell Adhesion Molecule TMIGD1 Is Downregulated in Advanced Colorectal Adenocarcinoma

Nicholas Woolf, Rosana D Meyer, Philip A Bondzie, Huihong Xu, Nader Rahimi. Boston University School of Medicine, Boston, MA.

Background: Colorectal cancer (CRC) is one of the most common malignancies and leading causes of cancer mortality. Although inactivation of adenomatous polyposis coli $(A P C)$ gene is the most common and critical event in the initiation of CRC, other genetic and cellular mechanisms by which tumor cells sense their microenvironment have profound importance in deriving the progression of malignancy and evasion from chemotherapy. We have recently identified transmembrane and immunoglobulin domain containing 1 (TMIGD1), a previously unknown protein, as a novel adhesion molecule that regulates epithelial cell survival in response to oxidative cell injury. The overall goal of this study was to establish the expression profile of TMIGD1 and determine the molecular mechanism of its expression in CRC.

Design: To evaluate TMIGD1 expression in colorectal tumors, we performed immunohistochemistry on two human CRC tissue microarray slides containing a total of 166 specimens. The staining intensity of each specimen was scored independently by two pathologists. Staining was scored as 0 (negative, $\leq 5 \%$ cells positive), $1+(6-25 \%$ cells positive), $2+(26-50 \%$ cells positive), and $3+(>50 \%$ cells positive). The tumor differentiation was graded morphologically (grades 1,2 and 3 as well, moderately and poorly differentiated, respectively). The mean staining scores were compared using ANOVA with Tukey post-hoc test. To examine the molecular regulation of expression of TMIGD1, we cloned its promoter and used a promoter reporter assay to determine the specific region involved in the expression of TMIGD1.

Results: In the CRC tissue microarray specimens, normal adjacent tissue (NAT) $(\mathrm{n}=52)$ had a mean TMIGD1 staining score of 1.87 , and grade $1(\mathrm{n}=21), 2(\mathrm{n}=62)$ and $3(\mathrm{n}=29)$ tumors had mean scores of 2.24, 1.52 and 1.14, respectively; expression of TMIGD1 was significantly higher in both NAT and well differentiated grade 1 tumors than in grade 3 tumors $(\mathrm{P}<0.01)$. Additionally, we have identified an approximately 100 base pair sequence on the 5 ' flanking end of the TMIGD1 promoter involved in the regulation of TMIGD1 expression.

Conclusions: TMIGD1 is expressed in human colonic epithelial cells. There is an inverse correlation between TMIGD1 expression and the grade of CRC. TMIGD1 expression was relatively high in both NAT and well-differentiated CRC; however, it was significantly reduced in poorly differentiated advanced CRC. Promoter analysis demonstrated that the presence of a 100 base pair sequence on the TMIGD1 promoter is required for expression. TMIGD1 is a potential biomarker in advanced CRC.

833 Mutational Landscape of BRAF-Mutated Microsatellite-Stable Colorectal Cancer Shows High Prevalence of Concurrent TP53 Mutations Elizabeth Yiru Wu, Fei Dong, Neal I Lindeman, Amitabh Srivastava. Brigham and Women's Hospital, Boston, MA.

Background: Colorectal carcinogenesis occurs through two distinct pathways: the conventional adenoma pathway characterized by mutations in APC, KRAS, SMAD4, and TP53, and the serrated pathway with BRAF and KRAS mutations along with CpG island hypermethylation and/or microsatellite instability (MSI-H). However, a subset of BRAF-mutated colorectal adenocarcinomas (CRCs) are microsatellite stable (MSS) and associated with adverse prognosis. The aim of our study was to analyze the mutational landscape of BRAF mutated MSS CRCs to determine the prevalence of concurrent mutations that may be the driving factors underlying the prognosis of these tumors. Design: Next generation sequencing (Oncopanel) was performed using a gene panel targeting all exons and select introns of 300 cancer related genes. Biopsy and resection specimens of microsatellite stable CRCs carrying BRAF mutations were identified using the Oncology Data Retrieval System at our institution. The results were previously curated based on clinical importance into one of four tiers. We only analyzed tier 1 (clinically actionable with a FDA-approved drug) to tier 3 (known relevance in tumorigenesis) mutations in the commonly mutated CRC genes for this study.

Results: 52 cases of BRAF (V600E) mutated, microsatellite stable colorectal adenocarcinomas ( $\mathrm{M}: \mathrm{F}=1.3: 1$; age range: $34-86 \mathrm{yrs}$; median age $56.5 \mathrm{yrs}$ ) formed the final study group. $15 / 52$ patients $(29 \%)$ had metastatic disease at initial presentation. In primary carcinomas with known location, 30/44 (68\%) were right sided tumors. Interestingly, TP53 mutations were highly prevalent $(36 / 52=69 \%)$ in this subset of BRAF mutant MSS CRC, as were other mutations commonly associated with the chromosomal instability pathway such as APC $(16 / 52=31 \%)$ and SMAD4 $(8 / 52=15 \%)$. Additionally, concurrent PIK3CA mutations were also present in a significant subset of these tumors $(8 / 52=15 \%)$

Conclusions: We identified concurrent mutations in genes commonly seen in the chromosomal instability pathway such as APC, TP53, and SMAD4, as well as actionable mutations in PIK3CA in our series of BRAF mutant MSS CRC. The high prevalence of concurrent TP53 mutations may be an important contributor underlying the poor prognosis of these tumors.

\section{Gastrointestinal Histopathologic Manifestations in Patients} with Common Variable Immunodeficiency (CVID)

Xinyu Wu, Romulo Celli, Dhanpat Jain, Xuchen Zhang. Yale University, New Haven, CT. Background: CVID is the second most common primary immunodeficiency syndrome, characterized by the combination of humoral and cell-mediated deficiency. GI involvement in CVID with symptomatic disease is common, often leading to GI biopsies. Some pathologic features have been reported previously, however, the evolution of these changes are unclear. The goal of this study was to assess histopathologic changes and natural evolution history in biopsies from various segment of GI tract in patients with CVID. 
Design: 44 GI biopsies from 15 patients with CVID over a 20-year period were reviewed, including biopsies from esophagus, stomach, duodenum, and colon. Detailed histologic features in each case were reviewed.

Results: The study included 5 males and 12 females, age range 9-80 (mean 45) years. Esophageal biopsies showed lymphocytic esophagitis (LyE) ( $n=9,67 \%)$, ulceration $(\mathrm{n}=2,22 \%)$ and candida esophagitis $(\mathrm{n}=1,11 \%)$. Decreased plasma cells, predominantly in the duodenum and colon, were seen in $85 \%$ of cases. Villous blunting mimicking celiac disease $(\mathrm{CD})$ was seen in $7(54 \%)$ patients, however, there was no increase in intraepithelial lymphocytes (IEL). Lymphoid aggregates were found in 5 duodenal $(39 \%), 10$ colonic $(77 \%)$ and 5 gastric $(50 \%)$ biopsies. Increased apoptotic bodies near the crypt bases ( 3 duodenum, 3 colon) mimicking GVHD were found in $23 \%$ of patients. Features similar to lymphocytic colitis (LC) were seen in $80 \%$ of patients (either paucicellular or typical), but plasma cells were absent in all of these patients. 2 patients showed eosinophilic enteritis that lasted for 5 years. Active chronic colitis mimicking ulcerative colitis was seen in $3(23 \%)$ patients. Of these 3 patients showed mild changes initially, but developed severe IBD-like colitis 3 years later. 3 patients showed normal colonic mucosa at initial biopsy, and developed GVHD-like changes 1 year later. In addition, 1 patient developed gastric adenocarcinoma and 2 patients developed extra-GI lymphoma.

Conclusions: The GI tract shows a wide range of histologic changes in CVID patients that resemble LyE, LC, CD, GVHD and IBD. The pathologic changes are dynamic. Significant decrease or absence of plasma cells in GI biopsies, sometimes in the presence of lymphoid aggregates is helpful in suggesting the possibility of CVID; however, in $15 \%$ of patients lamina propria plasma cell numbers appear normal. Lack of IEL increase further helps in differentiating CVID from CD in patients showing villous blunting. Increased risk of malignancies in CVID should also be recognized.

835 KRAS and VEGF Gene 3'-UTR Single Nucleotide Polymorphisms Predicted Susceptibility in Colorectal Cancer

Xiuli Xiao, Minnan Yang, Xin Li, Tian Xia, Hanan Long. The Affiliated Hospital of Southwest Medical University, Luzhou, Sichuan, China; Chengdu University of Traditional Chinese Medicine, Chengdu, Sichuan, China.

Background: Single nucleotide polymorphisms (SNPs) in tumor-related genes have been reported to play important roles in cancer development. Recent studies have showed that 3'-UTR polymorphisms are associated with the occurrence and prognosis of cancers. The aim of this study is to analyze the association between KRAS and VEGF gene 3'-UTR SNPs and genetic susceptibility of colorectal cancer (CRC).

Design: In this case-control study of $371 \mathrm{CRC}$ cases and 246 healthy controls, we analyzed the association between one SNP (rs1137188G $>$ A) in the KRAS gene and four SNPs (rs3025039C $>$ T, rs3025040C $>$ T, rs3025053G $>$ A and rs10434A $>$ G) in the VEGF gene and CRC susceptibility by the improved multiplex ligase detection reaction (iMLDR) method. We checked the selected SNPs' minor allele frequency and its distribution in the frequency of Chinese people by Hap-map database and HardyWeinberg equilibrium, and used multivariate logistic regression models to estimate odds ratios (ORs) and $95 \%$ confidence intervals (95\% CIs).

Results: We found that only the rs3025039C variant genotype in the VEGF gene was associated with a significant protection for CRC (adjusted OR $=0.696,95 \% \mathrm{CI}=$ $0.489-0.992 ; p=0.044$ for CC and CT $+\mathrm{TT}$ ). However, these were not found for the other SNPs (rs1137188GG, rs3025053AA and rs10434AA). In genetic polymorphisms analysis, we found that the KRAS rs1137188 variant AA genotype was more evident in colon and had higher portion of tumor size $\geq 5 \mathrm{~cm}$, and suggested that the $\mathrm{rs} 1137188$ variant AA genotype was associated with significantly increased risk of CRC patients $(P<0.05)$. The rs3025039 variant CC genotype was more evident in colon, tumor size $<5 \mathrm{~cm}$ and clinical stages III and IV.

Conclusions: Our study suggested that KRAS rs1137188 and VEGF rs3025039 may predispose patients to CRC and they may play important roles in the development of CRC.

836 Patterns of Injury in Esophageal Candidiasis: Clinical, Endoscopic, and Pathologic Correlations

Dongmei Xing, Rhonda K Yantiss, Jose Jessurun. New York Presbyterian-Weill Cornell Medicine, New York, NY.

Background: Candida is the most frequent cause of infectious esophagitis which may be seen in immunosuppressed (IS) as well as immunocompetent (IC) patients. Plaques and patchy or diffuse exudates are the characteristic endoscopic findings. We have noted variable inflammatory patterns in biopsy specimens from patients with candidal esophagitis, and performed this study to determine whether they correlate with clinical features, endoscopy findings, and immune status of the patients.

Design: We reviewed 70 consecutive esophageal biopsies that contained Candida. Each biopsy was assessed for the presence and type of inflammatory reaction and classified as non-inflammatory and inflammatory; the distribution and type of inflammation was noted in the latter group. Histologic findings were correlated with clinical features, endoscopic appearance and immune status of patients. A Fisher exact test was used for statistical analysis.

Results: All of the study patients were adults (mean age: 56 years, $\mathrm{M} / \mathrm{F}=5 / 9$ ). Thirty-two $(46 \%)$ were IS due to HIV/AIDS $(n=5)$, chemotherapy $(n=13)$, and immunosuppressive treatment $(\mathrm{n}=14)$. Endoscopic abnormalities were noted in $35(50 \%)$ patients. Seventeen (24\%) had biopsies with parakeratosis, invasive fungi and keratin debris, but no inflammation. Nine (53\%) were IS. Within this group, Candidiasis was endoscopically suspected in similar numbers of IS and IC patients. Most biopsies with histologic inflammation contained superficially oriented neutrophils $(34,49 \%) ; 15(21 \%)$ had, in addition, numerous lymphocytes, and $4(6 \%)$ only lymphocyte. Histologic inflammation correlated with endoscopic evidence of candidiasis in $60 \%$ IC individuals, but only $26 \%$ of IS patients $(\mathrm{p}=0.02)$. Interestingly, four patients with a lymphocyte-predominant infiltrate had achalasia $(n=2)$ or scleroderma $(n=2)$.
Conclusions: Classic endoscopic features of candidal esophagitis are present approximately $50 \%$ of all patients but only in $26 \%$ of IS patients with histologic inflammation. In addition to the well-known neutrophil-rich inflammation, abundant intraepithelial lymphocytes may be present. The latter inflammatory pattern was seen in patients with dysmotility disorders. Nearly $25 \%$ of cases lack mucosal inflammation but showed parakeratosis, invasive fungi, and detached debris containing fungi. No correlation was found between these inflammatory / reactive patterns and the immune status of the patients.

837 Medullary-Type Ampullary Carcinomas

Yue Xue, Gabriel Sica, Takashi Muraki, Bahar Memis, Serdar Balci, Cynthia Cohen, Brian Quigley, Alyssa Krasinskas, Michelle D Reid, Volkan Adsay. Emory University Hospital, Atlanta, GA.

Background: Programmed death-ligand 1 (PD-L1) expression by tumors is a mechanism by which tumors can evade the immune response and therefore this pathway is a target for immunotherapy. Since colorectal carcinomas with microsatellite instability preferentially express PD-L1 (PMID: 27198569), we investigated PD-L1 expression in mismatch-repair-deficient (MMR-D) and medullary-type ampullary carcinomas (AC). Design: Immunohistochemical stain for PD-L1 was performed on 19 cases of MMR-D AC (identified in a review of $125 \mathrm{ACs}$ ) as well as 8 medullary carcinomas of ampulla. PD-L1 expression in $>1 \%$ of tumor cells with $2+$ intensity (PMID: 27443512 ) was considered positive.

Results: PD-L1 expression was seen in 6 of 8 medullary carcinomas ( 4 of which also had MMR-D) but none of the 19 non-medullary MMR-D carcinomas showed PD-L1 positivity $(\mathrm{p}<0.001)$. The PD-L1 expresser carcinomas (PD-L1 +ve) had the following characteristics as opposed to PD-L1 negative (PD-L1 -ve) group: larger invasive size $(4.3 \mathrm{~cm}$ vs $2.3 \mathrm{~cm}, \mathrm{p}=0.04)$, BRAF mutation $(\mathrm{p}=0.02)$, and tumor-infiltrating lymphocytes (IELs, $\mathrm{p}=0.04$ ). PD-L1 +ve AC were also associated with tumor budding and poor prognosis (1-, 3- year survival of PD-L1 +ve vs PD-L1 -ve: 75\%, 75\% vs $94 \%, 88 \%$; respectively) although this did not reach statistical significance $(\mathrm{p}=0.08$ and $\mathrm{p}=0.79$ respectively).

Conclusions: PD-L1 expression is seen in $75 \%$ of medullary carcinomas (many of which are MMR-D as well) but not in non-medullary MMR-D AC. As such, PD-L1 expression is associated with increased tumor-infiltrating lymphocytes, larger invasive size and BRAF mutation. Accordingly, ACs of medullary type ought to be recognized as potential candidates for PD-1/PD-L1 immunotherapy.

838 Duodenal Neoplasm of Gastric Phenotype: An Immunohistochemical and Genetic Study with a Practical Approach to the Classification

Hidetaka Yamamoto, Risa Hida, Minako Hirahashi, Toshihiro Gi, Yoshinao Oda. Kyushu University, Fukuoka, Japan; University of Miyazaki, Miyazaki, Japan.

Background: Duodenal neoplasm of gastric phenotype (DNGP) is very rare, and details of histopathological, genetic and biological features are still unclear. Gene mutations in GNAS, KRAS and APC have been reported in pyloric gland adenoma and fundic gland type neoplasm of the stomach.

Design: In this study, we encountered 16 cases of extra-ampullary DNGP from benign to malignant, and examined mucin immunoprofile and oncogene mutations (GNAS, KRAS, APC, BRAF and CTNNBI).

Results: The 16 DNGPs were histologically classified into 7 adenomas ( 5 pyloric gland adenomas and 2 foveolar-type adenomas), 6 neoplasms of uncertain malignant potential (NUMPs) and 3 invasive adenocarcinomas $(n=3)$. NUMPs consisted of slightly atypical epithelial cells with pale, eosinophilic or basophilic cytoplasm growing in anastomosing or branching glandular pattern often with expansile submucosal extension. In contrast to invasive adenocarcinoma, NUMPs lacked significant nuclear irregularity, desmoplastic stromal reaction, lymphovascular invasion and metastasis; the features were reminiscent to fundic gland type neoplasm of the stomach. Immunophenotypically, most NUMPs were predominantly positive for MUC6 with variable expression of pepsinogen-I, $\mathrm{H}^{+} \mathrm{K}^{+}$ATPase, Human Gastric Mucin and MUC5AC. Molecular analyses revealed the gene mutations of GNAS in $6 / 16$ cases $(38 \%)$ of DNGP [4/7 $(57 \%)$ adenomas, $1 / 6$ (16\%) NUMPs, $1 / 3$ (33\%) invasive adenocarcinomas] and $A P C$ in $4 / 15(27 \%)$ DNGPs $[0 / 7(0 \%)$ adenomas, $2 / 6(33 \%)$ NUMPs, $2 / 3(67 \%)$ invasive adenocarcinomas]. BRAF mutation was present in only one (16\%) NUMP, and KRAS or CTNNB1 mutationin was absent.

Conclusions: In conclusion, gastric-phenotypic adenoma and NUMP of the duodenum are similar to each counterpart of the stomach in terms of histological, genetic and clinicopathological features. We would like to propose a term of NUMP as an intermediate category between adenoma and invasive adenocarcinoma. Our results may provide novel insights into the classification of undescribed but distinctive duodenal tumors showing analogy with gastric phenotypic neoplasms of the stomach.

839 Diagnostic Utility of SATB2 in Gastrointestinal Poorly Differentiated Adenocarcinomas with Signet Ring Cells, Pure Signet Ring Cell Carcinomas and Goblet Cell Carcinoids

Chen Yang, Lingxin Zhang, Dengfeng Cao. Washington University School of Medicine, St. Louis, MO.

Background: SATB2 has been shown to be a sensitive diagnostic marker for colorectal adenocarcinomas (CRAs). However reported studies focused only on glandularforming CRAs and no study has tested SATB2 in gastrointestinal poorly differentiated adenocarcinomas with signet ring cell feature (PDA-SRCs), pure signet ring cell carcinomas (PSRCs) and gobet cell carcinoids (GCCs). Here we investigated SATB2 immunoreactivity in these tumors and compared its diagnostic utility to CDX2. 
Design: Tissue microarrays were constructed using $3 \mathrm{~mm}$ core tissue ( 3 cores per case) and consisted of 71 cases (18 gastroesophageal PDA-SRCs/PSRCs, 4 smal intestinal PSRCs, 26 colorectal PDA-SRCs and 3 PSRCs, 3 appendiceal PDA-SRCs, 17 appendiceal GCCs). Immunohistochemical stainings for SATB2 and CDX2 were performed. Only nuclear staining was considered positive for these two markers. The stainings were scored as $0(<1 \%$ cells $), 1+(1-25 \%), 2+(26-50 \%), 3+(51-75 \%), 4+$ $(>75 \%)$.

Results: All 18 gastroesophageal and 4 small intestinal PDA-SRCs and PSRCs were negative for SATB2 but they showed positive CDX2 staining in 21/22 cases. SATB2 was positive in 18/26 colonic PDA-SRCs and in 1/3 PSRCs but all these 29 cases were positive for CDX2. Among the 18/26 SATB2-positive colonic PDA-SRCs, SATB2 staining was lost in the SRC component but retained in the non-SRC component in 7 $(39 \%)$, less SATB2 positivity in the SRC component than the non-SRC component in $6(33 \%)$, similar SATB2 positivity in the SRC and non-SRC components in $5(28 \%)$. SATB2 and CDX2 were both positive in 2/3 appendiceal PDA-SRC. All 17 appendiceal GCCs were positive for both SATB2 and CDX2. Detailed comparison of SATB2 to CDX2 was summarized in Table 1.

\begin{tabular}{|l|c|c|}
\hline Tumor origin & SATB2 & CDX2 \\
\hline $\begin{array}{l}\text { Gastroesophageal and small } \\
\text { intestinal (N=22) }\end{array}$ & $0 / 22$ & $\begin{array}{c}21 / 22(2+\text { in } 2,3+ \\
\text { in } 1,4+\text { in } 18)\end{array}$ \\
\hline Colorectal PDA-SRCs (N=26) & $\begin{array}{c}18 / 26 \text { in non-SRC component: }(1+\text { in } 5, \\
2+\text { in 3, 3+ in 3, 4+ in 7) } \\
11 / 26 \text { in SRC component }(1+\text { in } 6,2+ \\
\text { in 4, 4+ in } 1)\end{array}$ & $\begin{array}{c}26 / 26(3+\text { in } 2,4+ \\
\text { in 24) }\end{array}$ \\
\hline Colorectal PSRCs (N=3) & $1 / 3(1+)$ & $3 / 3(4+$ in 3) \\
\hline Appendiceal PDA-SCRs (N=3) & $2 / 3(4+$ in 2$)$ & $2 / 3(4+$ in 2) \\
\hline $\begin{array}{l}\text { Appendiceal goblet cell } \\
\text { carcinoids (N=17) }\end{array}$ & $17 / 17(2+$ in 2, 4+ in 15) & $17 / 17(4+$ in 17) \\
\hline
\end{tabular}

Conclusions: SATB2 was negative in the upper GI PDA-SRCs/PSRCs. In colorectal PDA-SRCs and PSRCs, SATB2 was not as sensitive as CDX2 and showed decreased immunoreactivity with decreasing differentiation. SATB2 showed similar high sensitivity as CDX2 in goblet cell carcinoids.

\section{0}

Morphological and Molecular Features of Gastric Glomus

\section{Tumors}

Dongwei Zhang, Sarawut Kongkarnka, Elena V Komissarova, Armando Del Portillo, Antonio G Neto, Jorge Sepulveda, Antonia Sepulveda. Columbia University Medical Center, New York, NY; NYU Langone Medical Center, New York, NY.

Background: Glomus tumors are rare lesions that arise from the neuro-myoarterial canal or glomus body that is responsible for thermoregulation. Most glomus tumors occur in the peripheral soft tissue, especially in digits, palms, and soles. Gastric glomus tumors (GGTs) are extremely rare and the pathogenesis and molecular genomic alterations are not well studied.

Design: We analyzed six cases of GGTs resected between 2000 and 2016 . Clinicopathological features were reviewed and immunohistochemical studies were performed. Next generation sequencing (NGS) was done in three cases to screen for mutations in a panel of 467 cancer-related genes in the Columbia University Comprehensive Cancer panel (CCCP). Tissue macro-dissection was performed to enrich for tumor tissue (at least $80 \%$ ) for DNA extraction. All 6 cases were tested for $B R A F \mathrm{~V} 600 \mathrm{E}$ and $K R A S$ exon 2 mutations by PCR and Sanger sequencing.

Results: The mean age of the 6 patients was 57.3 years (range: $45-72$ ), with a male predominance ( 4 males and 2 females). All tumors occurred in the distal stomach. The mean size of the tumors was $3.5 \mathrm{~cm}$ (range: 2.5-4.8). Histologically, the tumors showed nests of uniform, round epithelioid-appearing cells associated with ectatic vessels. No necrosis, pleomorphism or significant mitotic activity was seen and Ki-67 proliferation index was low (1-5\%). Immunohistochemically, the tumors were positive for smooth muscle actin, caldesmon, muscle-specific actin, and synaptophysin and were negative for cytokeratin, C-kit, DOG-1, chromogranin, CD56, calponin, and S100. No lymphovascular invasion or lymph node metastasis was identified in any of the tumors. No $B R A F$ or $K R A S$ mutations were detected by single gene sequence analysis in the 6 cases. NGS revealed heterozygous germline non-synonymous single-nucleotide variants (SNVs) on genes associated with multiple neuroendocrine neoplasia (RET or $M E N 1$ ) in the three cases sequenced with CCCP (case1: RET G446R in exon 7; case 2: RET S649L in exon 11; case 3: MEN1 P545S in exon 10). Non-synonymous SNVs were also detected on other cancer-related genes such as $A T M, M A P K 2, I D H 2, P M L$, SHQ1, and NOTCH2.

Conclusions: This is the first report of extensive mutational analyses of GGTs. Our data show that somatic mutations do not appear to drive GGTs. However, nonsynonymous SNVs leading to possible functional changes in cancer-related genes, especially genes associated with multiple neuroendocrine neoplasia, may contribute to the tumorigenesis of GGTs.

\section{Profiling of Metastatic Colorectal Cancers Identifies Potential} Responders to Targeted Therapy

Lei Zhang, Weiguo Liu, Wilfrido D Mojica. SUNY at the University at Buffalo, Buffalo, NY.

Background: Metastasis of colorectal adenocarcinoma (CRC) is a problem that affects approximately half of the patients with the disease and this needs to be addressed. Advances in the field of antibody drug conjugate (ADC) therapy may be a promising approach in that directed therapy can be administered to specifically target metastatic tumor cells. ADCs are comprised of a monoclonal antibody linked to a toxic carrier payload. There are a number of ADCs that have either been recently introduced to the clinic, are in clinical trials, or in development. Refining their use requires identifying targets in metastatic tumor cells. In this study, a panel of antibodies to plasma membrane proteins $(\mathrm{PMbP})$ which have been reported to be expressed more in tumor cells than normal cells were tested on cases of metastatic CRC to determine the utility of a profiling approach to differentiate potential responders from non-responders to ADC based adjuvant therapy.

Design: Eleven cases of CRC were identified and blocks consisting of lymph nodes with metastatic disease were tested against a panel consisting of five antibodies (PTK-7, Trop-2, AXL, CDH2, and L1CAM) directed to these PMbPs. Antigen retrieval using an autoclave and conventional immunohistochemical methods were performed to optimize conditions and later test on the tissue sections. The resulting stained slides were evaluated to determine plasma membrane expression $(\mathrm{PMbE})$. A case was considered positive if $>25 \%$ of the tumor cell population demonstrated $\mathrm{PMbE}$.

Results: In all 11 cases, membranous staining restricted to the luminal surface of the neoplastic glands was noted with the PTK-7 antibody. Seven of the 11 cases $(64 \%)$ stained with L1CAM exhibited partial to circumferential membranous staining in $>25 \%$ of the tumor cells. Only staining of the desmoplastic stroma was noted with the antibody to the protein AXL. Membranous staining was not identified with either the $\mathrm{CDH} 2$ or Trop- 2 antibody.

Conclusions: ADCs with their monoclonal antibody component directed to either PTK-7 or L1CAM would find utility, either alone or in combination, in the majority of the CRC cases tested. The absence of L1CAM staining in 4 of the 11 cases confirms the need to profile the tumor tissue to identify potential non-responders.

$842 \quad$ APC and KRAS Genetic Variants Associated with Colorectal Cancer Histology Grade and Tumor Staging

Wei Zhang, Kyle Gettler, Aneta Waluszko, Tatyana Sidorenko, Gordana Katava, David Zhang, Fei Ye. Mount Sinai Health System, New York, NY; Yale University School of Medicine, New Haven, CT.

Background: Colorectal cancer (CRC) is the fourth leading cause of cancer deaths worldwide. Next generation sequencing (NGS) has been widely used for detecting somatic mutation in clinical CRC management. This study is to characterize the association of CRC specific somatic mutation with tumor grade and staging.

Design: We reviewed all CRC cases that have next-generation sequencing results at Mount Sinai Health System. To evaluate the staging status, total 111 cases with surgical resection specimen and adequate clinical information are included in this study. The demographic information, specimen, tumor site, tumor grade, TNM and AJCC staging are obtained from the Pathology report and medical record. NGS was done on The Ion Personal Genome Machine $\left(\mathrm{PGM}^{\mathrm{TM}}\right)$ sequencer by using Ion Torrent ${ }^{\mathrm{TM}} \mathrm{AmpliSeq}^{\mathrm{TM}}$ Cancer Hotspot Panel v2. The mean depth across various amplicons is $2,000 \times$, and $200 \times$ nucleic acid coverage and $5 \%$ of somatic gene mutation are used as the cutoff to make the final variant call. All somatic mutations with frequency higher than $2 \%$ were included in the final analysis. For statistical analysis, we initially run the logistic regression on the full dataset, and then use stepwise regression to predict the best model. Results: The age of these 111 cases is ranging from 36 to 90 years old with mean age at 62.5 years. Total 144 different mutations are detected in 30 genes, which include APC, BRAF, CTNNB1, FBXW7, IDH1, KRAS, PIK3CA, SMAD4, STK11, SMARCB1, TP53, and etc. Stepwise analysis reveals that the two variants, APC_R1450* and KRAS G12C, appeared to be significantly associated with tumor histological grade $(\mathrm{p}<0.05)$; the variants, APC_R1450*, and TP53_R248W appeared to be significantly associated with Tumor T stage $(\mathrm{p}<0.05)$.

\begin{tabular}{|l|l|l|l|}
\hline Genetic Variant & Allele Frequency (\%) & Tumor Histology Grade & T Stage \\
\hline KRAS_G13D & 5.41 & $\mathrm{n} / \mathrm{a}$ & 0.136 \\
\hline APC_R1450* & 4.50 & 0.046 & 0.044 \\
\hline KRAS_G12V & 3.60 & $\mathrm{n} / \mathrm{a}$ & 0.174 \\
\hline PIK3CA_E545K & 3.60 & $\mathrm{n} / \mathrm{a}$ & 0.072 \\
\hline TP53_R248W & 2.70 & $\mathrm{n} / \mathrm{a}$ & 0.031 \\
\hline APC_R876* & 2.25 & 0.063 & $\mathrm{n} / \mathrm{a}$ \\
\hline KRAS_A146T & 2.25 & $\mathrm{n} / \mathrm{a}$ & 0.185 \\
\hline KRAS_G12C & 2.25 & 0.044 & $\mathrm{n} / \mathrm{a}$ \\
\hline
\end{tabular}

Conclusions: APC, KRAS, and TP53 are well-known important genes in the CRC etiology pathway. This study confirmed that certain mutations in these genes are associated with tumor histology grade and staging. Sequencing a larger CRC cohort may aid us develop a risk model that can predict tumor histology grade and staging.

\section{Fibromuscular Strictures in lleal Crohn's Disease: A Detailed} Morphometric and Histopathologic Analysis

Xiaofei Zhang, Huai-Bin Mabel Ko, Zhenjian Cai, Hongfa Zhu, Alexandros D Polydorides, Joana Torres, Judy Cho, Bachir Taouli, Noam Harpaz. Icahn School of Medicine at Mount Sinai, New York, NY.

Background: The pathogenesis of intestinal stricturing, one of the chief complications of Crohn's disease, is poorly understood and little progress has been made in its prevention or therapy. Although such strictures are conventionally described as fibrostenotic, expansion of the intestinal smooth muscle has also been implicated. We aimed to detail the histological and morphometric contributions and distributions of fibrous and smooth muscle tissue in individual mural layers of Crohn's ileal strictures. Design: We retrospectively analyzed 37 ileal strictures confirmed by magnetic resonance enterography and resected from patients with a clinical diagnosis of Crohn's disease. Sections of the strictures and of adjacent non-strictured control segments were stained with H\&E, anti-smooth muscle actin and Sirius red. The corresponding digital scanned images were evaluated morphometrically using Halo morphometry software (v1.4, Indica Labs). 
Results: Strictures were characterized by a mean 2.3 -fold increase in overall mural thickness and by 2.6 - and 1.8 -fold increases in the areas occupied by smooth muscle and collagen, respectively. Approximately half the increase in wall thickness was attributable to a mean 22-fold expansion of the muscularis mucosae (MM) from $2.2 \pm 1.6 \%$ to $16.5 \pm 10.1 \%$ of the total mural thickness and the remainder of the increase to expansions of the internal layer of muscularis propria (MP-I) and submucosa (SM). The external layer of the muscularis propria was similar in thickness and composition to controls. Microscopically, the expanded MM featured (1) architectural disarray, (2) myocyte hyperplasia and (3) pericellular collagen fibers. In contrast, the thickened MP-I featured (1) preserved muscular architecture, (2) no pericellular collagen fibers, and (3) widened intramuscular septa with increased collagen. The SM exhibited fibrosis in continuity with the intramuscular septa. Additionally, submucosal arteries and veins frequently exhibited eccentric fibromuscular hyperplasia which was polarized toward the mucosa. Conclusions: Stricturing Crohn's ileitis is characterized by distinctive fibromuscular alterations in the individual intestinal wall layers. These alterations may reflect differential mesenchymal responses to luminal-based and transmural inflammatory stimuli. The implications for stricture pathogenesis may help guide progress toward prevention and therapy.

\section{$844 \quad$ Constrictive Strictures in Crohn's lleitis}

Xiaofei Zhang, Huai-Bin Mabel Ko, Zhenjian Cai, Hongfa Zhu, Alexandros D Polydorides, Noam Harpaz. Icahn School of Medicine at Mount Sinai, New York, NY. Background: Ileal strictures in Crohn's disease (CD) classically present as segmental regions of mural thickening, "rubber-hose" rigidity and luminal narrowing, features that correspond microscopically to fibromuscular, lymphoid or neural hypertrophy and distorted mural architecture. We describe a subset of ileal CD strictures in which these features are relatively inconspicuous and where luminal narrowing results mostly from contracture of the intestinal wall.

Design: In a prospective study, resected ileal strictures of $26 \mathrm{CD}$ patients were serially cross-sectioned along with the adjoining non-strictured bowel. Evaluation of H\&Estained slides revealed a subset of strictures with reduced external circumference. For morphometric analysis we selected a specimen that contained 5 separate strictures yielding a total of 79 cross-sections (Figure). H\&E and smooth muscle actin-stained slides were digitally scanned at $20 \mathrm{X}$ and evaluated morphometrically via Halo software (Indica Labs).

Results: The outer circumferences of the intestinal wall in strictured segments at their narrowest points measured $58.1,72.1,63.0,69.4$ and $66.2 \%$ of controls. The walls of the strictured segments and individual muscular layers were thickened compared to control sections (mean $6.4 \pm 2.2$ vs $3.6 \pm 0.9 \mathrm{~mm}$, respectively, $\mathrm{p}=0.004$ ) but there were no significant differences in the areas of the muscularis mucosae $(11.3 \pm 2.0 \mathrm{vs}$. $14.3 \pm 7.2 \mathrm{~mm}^{2}$, respectively, $\left.\mathrm{p}=0.77\right)$ or muscularis propria $\left(75.1 \pm 10.0\right.$ vs. $65.1 \pm 1.7 \mathrm{~mm}^{2}$, respectively, $\mathrm{p}=0.69)$, suggesting a net conservation of muscle mass. There was no histological evidence of architectural distortion of the mural layers or substantial neural or lymphoid hyperplasia. The maximal proportions of outer circumferences covered by mesenteric fat in the strictured and non-strictured areas were $79.8 \pm 5.6 \%$ vs. $42.5 \pm 4.9 \%$, respectively $(\mathrm{p}=0.004)$.

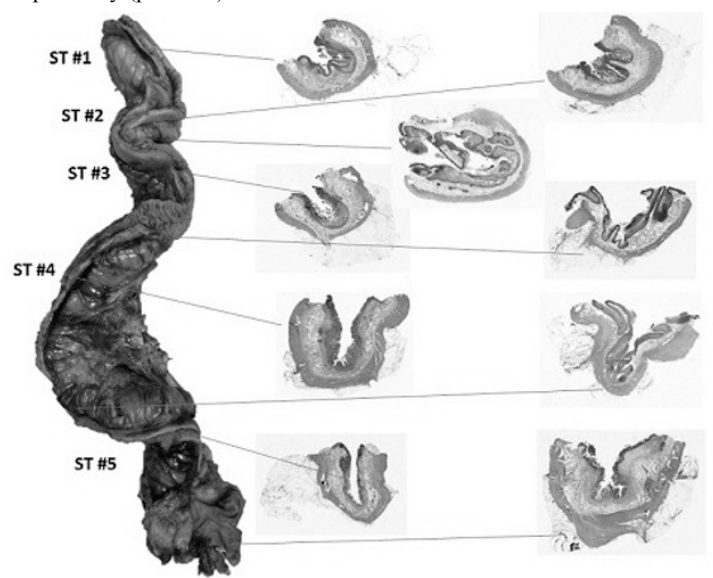

Conclusions: A subset of "constrictive" ileal strictures in CD is characterized by contracture, preserved mural architecture and near-encasement by mesenteric fat. We hypothesize that such strictures result from compression by hypertrophic mesentery, but cannot exclude a role for unidentified physiologic interactions between the mesentery and intestinal wall. These findings support recent hypotheses regarding a primary role for the mesentery in the pathogenesis of $\mathrm{CD}$.

\section{Genitourinary Pathology (including Renal tumors)}

845 Prostate Cancer

Daniel W Abbott, Shira Ronen, Amrou Abdelkader, Anjishnu Banerjee, Yayun Xu, Oleksandr Kravtsov, Ken A Iczkowski. Medical College of Wisconsin, Milwaukee, WI. Background: The presence and amount of cribriform prostate cancer (Cribr.) versus other Gleason 4 cancer are associated with increased recurrence (PMID:21685037) and cancer death (PMID:25189638). PTEN loss inactivates cell cycle inhibitor p27/ Kip1 (PMID:11175795): both prognostically adverse events, whose correlation with morphology has not been studied in detail.

Design: Selected whole slides from 46 cancer specimens with Cribr. were immunostained (IHC) for PTEN or p27. A subset of 15 cases was studied by chromogenic in situ hybridization (ISH, RNAscope ${ }^{\circledR}$, Advanced Cell Diag.) using probe to PTEN or CDKN1B (to detect p27). To analyze both IHC and ISH, for each Gleason (G1.) morphologic subtype, 2-3 JPG 400x images were captured, and (after editing out stroma) the fraction of tumor epithelium positive was digitally assessed using Axiovision Release 4.7.1. For IHC, $0-0.1$ was assigned $0,>0.1-0.4$ was $1+,>0.4-0.7$ was $2+,>0.7$ was $3+$. Statistical analysis for IHC used the Kolmogorov-Smirnov test (10 comparisons, significant if $\mathrm{p}<0.005)$. ISH used fixed effects for morphology and random effects for each patient (significant: $\mathrm{p}<0.05$ ).

Results: $\underline{\mathrm{IHC}}$ :

\begin{tabular}{|l|l|l|}
\hline Acini & Mean PTEN IHC $(0-3+)$ & $\mathrm{p}$ \\
\hline Benign & 2.52 & ref \\
\hline Gl. 3 & 1.88 & .07 \\
\hline Gl. 4- Fused & 1.50 & .0012 \\
\hline Gl. 4 Cribr- peripheral cells & 1.48 & .01 (borderline) \\
\hline Gl. 4 Cribr- central cells & 0.90 & $<.0001$ \\
\hline Gl. 4 Cribr- small size like benign & 1.22 & .0003 \\
\hline Gl. 5 & 0.80 & $<.0001$ \\
\hline
\end{tabular}

p27 loss compared to benign acini (mean 2.5+) was significant only for Cribr- peripheral (mean 1.5+, $\mathrm{p}=.0029)$; Fused was $(\mathrm{p}=.0075)$.

ISH: With PTEN, significant loss normalized to benign acini was observed for cribriform cancer $(\mathrm{p}<0.02)$, but not for fused small acini or Gleason 3/separate acini.

\begin{tabular}{|l|l|l|}
\hline Acini & Mean PTEN ISH* & p \\
\hline Benign & 7.9 & ref \\
\hline Gl. 3 & 7.2 & .582 \\
\hline Gl. 4- Fused & 5.9 & .159 \\
\hline Gl. 4- Cribr (peripheral and central) & 5.0 & .019 \\
\hline \multicolumn{2}{|c|}{$* 100 x$ (Pixels of epithelium with signal/Total pixels of epithelium) } \\
\hline
\end{tabular}

With CDKN1B, the degree of signal loss in these 3 cancer morphologies was not significant.

Conclusions: The uniquely adverse outcome of cribriform cancer is mirrored by greater molecular changes than in other morphologies.

Cribriform structures' peripheral cells have higher proliferation index according to prior work. PTEN loss predominates in central cells and p27 loss peripherally, suggesting that a biphenotypic population plays a role in generating cribriform growth. For PTEN loss as a prognostic factor, cribriform structures demand separate assessment in peripheral vs. central populations.

846 Non-Tumoral Parenchymal Changes in Renal Cell Carcinoma Cases

Behnoush Abedi-Ardekani, Lars Egevad, Roz Banks, James D McKay, Paul Brennan, Ghislaine Scelo. International Agency for Research on Cancer, Lyon, France; Karolinska Institute, Stockholm, Sweden; University of Leeds, Leeds, United Kingdom.

Background: Large, unexplained geographical variations in renal cell carcinoma (RCC) incidence rates have been observed, with the highest rates observed in the Czech Republic (CR). We explored chronic pathological changes in the non-tumoral renal cortex (CPCRC) of RCC cases from five European countries involved in the Cancer Genomic of the Kidney (CAGEKID) study, and their possible relationship with tumorigenesis.

Design: Scanned images of 626 frozen normal tissue samples distant from the tumor in conventional RCC cases from CR, Romania, Serbia, United Kingdom (UK) and Russia were read twice by one pathologist who evaluated the degrees of interstitial inflammation and fibrosis, tubular atrophy, golomerulosclerosis and arterial wall thickening. In the CAGEKID project, an independent systematic review of normal tissues was conducted by another pathologist to assess the level of inflammation and fibrosis with additional comments for any other specific features such as glomerulosclerosis. The agreement between these two independent observations was $89 \%$. We used logistic regressions to analyze the association of these pathologies with clinico-pathological variables and country of residence.

Results: CPCRC were observed in $116(18.5 \%)$ of samples. Higher age was the strongest predictor $(\mathrm{OR}=1.06$ for 1-year increment, $95 \% \mathrm{CI}$ : 1.04-1.08). After adjusting for age, country was significantly associated with the presence of CPCRC. Compared to Russia, CPCRC were more common in $\mathrm{CR}(\mathrm{OR}=2.04,1.15-3.61)$ and in Romania $(\mathrm{OR}=3.56$, 1.80-7.07), while no significant difference was observed with the UK and Serbia. To assess potential confounders of the association with country and sampling method, we 